\title{
Uric acid, blood pressure, and gout management
}

Citation for published version (APA):

Scheepers, L. (2017). Uric acid, blood pressure, and gout management: Beneath the surface. [Doctoral Thesis, Maastricht University]. Maastricht University. https://doi.org/10.26481/dis.20170707ls

Document status and date:

Published: 01/01/2017

DOI:

10.26481/dis.20170707ls

Document Version:

Publisher's PDF, also known as Version of record

\section{Please check the document version of this publication:}

- A submitted manuscript is the version of the article upon submission and before peer-review. There can be important differences between the submitted version and the official published version of record.

People interested in the research are advised to contact the author for the final version of the publication, or visit the DOI to the publisher's website.

- The final author version and the galley proof are versions of the publication after peer review.

- The final published version features the final layout of the paper including the volume, issue and page numbers.

Link to publication

\footnotetext{
General rights rights.

- You may freely distribute the URL identifying the publication in the public portal. please follow below link for the End User Agreement:

www.umlib.nl/taverne-license

Take down policy

If you believe that this document breaches copyright please contact us at:

repository@maastrichtuniversity.nl

providing details and we will investigate your claim.
}

Copyright and moral rights for the publications made accessible in the public portal are retained by the authors and/or other copyright owners and it is a condition of accessing publications that users recognise and abide by the legal requirements associated with these

- Users may download and print one copy of any publication from the public portal for the purpose of private study or research.

- You may not further distribute the material or use it for any profit-making activity or commercial gain

If the publication is distributed under the terms of Article $25 \mathrm{fa}$ of the Dutch Copyright Act, indicated by the "Taverne" license above, 


\section{Uric acid, blood pressure, and gout}

management

Beneath the surface 
No part of this book may be reproduced or transmitted in any form or by any means, without prior permission in writing by the author, or when appropriate, by the publishers of the publications.

Cover artwork: An Inescapable Pressure | oil on canvas, $140 \mathrm{~cm} \times 120 \mathrm{~cm}, 2015$. From the project Crystallisation of Thought | An artistic interpretation of the disease Gout by artist Jill Tegan Doherty. Image @]ill Tegan Doherty 2017

"A collaboration between Prof. Michael Doherty and artist Jill Tegan Doherty, initiated to help raise awareness of the disease Gout. To bring to light that it is a disease which needs to be taken seriously. The project aims to break down the barriers to care and bring strength in knowledge to patients and potential sufferers."

Cover design: Remko Rinia, www.linecreation.nl

Layout: $\quad$ Tiny Wouters

Production: Gildeprint B.V.

ISBN/EAN: $\quad 9789462336537$

Financial support by the Dutch Heart Foundation for the publication of this thesis is gratefully acknowledged. 


\title{
Uric acid, blood pressure, and gout management
}

\author{
Beneath the surface
}

\begin{abstract}
PROEFSCHRIFT
ter verkrijging van de graad van doctor aan de Universiteit Maastricht, op gezag van de Rector Magnificus, Prof. dr. Rianne M. Letschert volgens het besluit van het College van Decanen, in het openbaar te verdedigen op vrijdag 7 juli 2017, om 16:00 uur.
\end{abstract}

door

Lieke Scheepers 


\section{Promotoren}

Prof. dr. A.E.R.C.H. Boonen

Prof. dr. ir. I.C.W. Arts

Prof. dr. C.D.A. Stehouwer

\section{Beoordelingscommissie}

Prof. dr. J.F.M. Metsemakers (voorzitter)

Dr. A. Dehghan (School of Public Health, Epidemiology \& Biostatistics, United Kingdom)

Prof. dr. L.T.H. Jacobsson (Sahlgrenska Academy, University of Gothenburg, Sweden)

Prof. dr. P.W. de Leeuw

Dr. S.J.R. Meex 


\section{CONTENTS}

Glossary of terms related to genetics or medication adherence

Chapter 1 General introduction

Part I Uric acid and blood pressure: the role of uric acid production

Chapter 2 Xanthine oxidase gene variants and their association with

blood pressure and incident hypertension: a population study

Chapter 3 Associations of plasma uric acid and purine metabolites with

blood pressure in children: the KOALA birth cohort study

Chapter $4 \quad$ Uric acid and blood pressure: exploring the role of uric acid

production in The Maastricht Study

Part II Gout management by the patient and general practitioner

Chapter 5 Medication adherence among gout patients initiated allopurinol: a retrospective cohort study in the Clinical Practice Research Datalink

Chapter 6 Medication adherence among patients with gout: a systematic 109 review and meta-analysis

Chapter $7 \quad$ Knowledge, illness perceptions and stated clinical practice behaviour in management of gout: a mixed methods study in general practice

Chapter 8 Summary and general discussion

Addendum

Valorisation

Samenvatting

Acknowledgments

Curriculum vitae

List of publications 

Glossary of terms related to genetics or medication adherence

\begin{tabular}{|c|c|}
\hline \multicolumn{2}{|l|}{ Genetic terms } \\
\hline Allele & A specific variation of a single-nucleotide polymorphism or gene. \\
\hline Enzyme & Molecules that accelerate, or catalyse, chemical reactions. \\
\hline Gene & $\begin{array}{l}\text { Comprises a DNA sequence, including introns, exons, and regulatory } \\
\text { regions, related to transcription of a given RNA. }\end{array}$ \\
\hline Genotype & $\begin{array}{l}\text { Part of a DNA sequence and determines a specific trait. If the alleles of the } \\
\text { chromosomes are the same, the genotype is called homozygous, if } \\
\text { different, heterozygous. }\end{array}$ \\
\hline Linkage disequilibrium (LD) & $\begin{array}{l}\text { Correlation between alleles at different loci within the population. The } \\
\text { term LD describes a state which is opposite to the hypothetical situation in } \\
\text { which all loci exhibit complete independence, called linkage equilibrium. }\end{array}$ \\
\hline Mendelian randomization studies & $\begin{array}{l}\text { In epidemiology, this is a method of using variation in a gene, as a proxy } \\
\text { for an exposure, to examine the causal effect of the modifiable exposure } \\
\text { on the disease. }\end{array}$ \\
\hline Phenotype & $\begin{array}{l}\text { Expression of characteristic of a trait, for example body height or blood } \\
\text { pressure. }\end{array}$ \\
\hline Pleiotropic effect & $\begin{array}{l}\text { Situation in which a single gene / polymorphism influences a variety of } \\
\text { traits (phenotypes) }\end{array}$ \\
\hline \multicolumn{2}{|l|}{ Medication adherence terms } \\
\hline $\begin{array}{l}\text { Adherence to medication (general } \\
\text { term) }\end{array}$ & $\begin{array}{l}\text { The process by which patients take their medications as prescribed, } \\
\text { including initiation, implementation and discontinuation. }\end{array}$ \\
\hline Adherence & $\begin{array}{l}\text { The extent to which a patient's actual medication intake corresponds to the } \\
\text { prescribed dosing regimen during observation time. }\end{array}$ \\
\hline Discontinuation & $\begin{array}{l}\text { Marks the end of therapy, when the next dose to be taken is omitted and } \\
\text { no more doses are taken thereafter or when the next dose is after the } \\
\text { permissible gap length (e.g. at least } 30 \text { days after finishing the last } \\
\text { prescription). }\end{array}$ \\
\hline Implementation & $\begin{array}{l}\text { The extent to which a patient's actual dosing corresponds to the prescribed } \\
\text { dosing regimen, from initiation until discontinuation. }\end{array}$ \\
\hline Initiation & The time from prescription until first dose is taken. \\
\hline Non-persistence & See Discontinuation \\
\hline Persistence & $\begin{array}{l}\text { Is the length of time between initiation and the last dose, which } \\
\text { immediately precedes discontinuation. }\end{array}$ \\
\hline $\begin{array}{l}\text { Time until discontinuation / non- } \\
\text { persistence }\end{array}$ & See Persistence \\
\hline
\end{tabular}





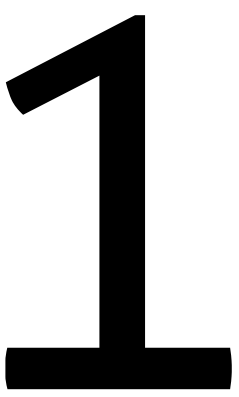

\section{General introduction}

Adapted from

'Overproducers' and 'underexcretors': the key to understanding the complex relation between uric acid and cardiovascular diseases?

Ilja Arts, Lieke Scheepers, José Wijnands, Pieter Dagnelie, Sjef van der Linden, Annelies Boonen 

General introduction $\mid 11$

\section{GENERAL INTRODUCTION}

Gout is the most prevalent form of inflammatory rheumatic disease, and is characterized by the deposition of monosodium urate crystals in the synovial fluid. ${ }^{1}$ Unlike other forms of arthritis, the pathogenesis of gout is well understood and effective treatments to reduce serum uric acid concentration below the saturation point of monosodium urate are available. ${ }^{2}$ If untreated, tophaceous gout can cause a severe inflammatory response leading to cartilage and bone destruction. ${ }^{3,4}$ In the long-term, it can result in impaired function and a decline in health-related quality of life. ${ }^{5}$ Although gout is considered a well treatable disease, it is often insufficiently controlled. Both patients and physicians factors can be barriers to optimal management. ${ }^{6,7}$ To improve gout management, insights into patient medication adherence and gout management by the general practitioner are essential.

Patients with gout have an increased risk of cardiovascular disease, which is often attributed to the hyperuricemia. ${ }^{8}$ With a prevalence of $20 \%$ asymptomatic hyperuricemia is far more prevalent than gout, ${ }^{9}$ and has indeed been associated with an increased risk for hypertension and cardiovascular diseases. ${ }^{10-12}$ However, there has been much debate on the causal nature of these associations. Some argue that elevated serum uric acid is just an innocent bystander in the pathogenesis of hypertension and cardiovascular diseases. From 2001 onwards, experimental studies have however established plausible mechanisms linking uric acid with the development of hypertension by using rat models. ${ }^{13,14}$ One insight derived from these studies assigns an important role to the generation of free radicals during the production of uric acid. So far, epidemiological studies have focused on the association between blood pressure and concentrations of uric acid in serum or plasma and have ignored the distinction between uric acid concentration and its production. ${ }^{12}$ Since the production of uric acid may contribute, independent of uric acid concentration, to the pathogenesis of hypertension, the production should be investigated as well.

This thesis comprises two main parts. In Part $I$, the association between uric acid and blood pressure, in particular the role of uric acid production is studied. In Part II, medication adherence in patients with gout requiring uric acid lowering therapy and the knowledge, illness perceptions and stated clinical practice behaviour of general practitioners when managing gout are addressed.

\section{Uric acid metabolism}

Uric acid is generated as part of the normal turnover of the purine nucleic acids adenine and guanine (Figure 1). The purine nucleotides are either derived from endogenous (DNA, RNA and ATP) or exogenous food-derived purines. During the last two steps of the purine metabolism, xanthine oxidoreductase (XOR) catalyses the breakdown of hypoxanthine to xanthine, and xanthine to uric acid. In most vertebrates uric acid is 
$12 \mid$ Chapter 1

further catabolized into the more soluble allantoin by the enzyme uricase, but humans and higher primates lack this enzyme. ${ }^{15}$

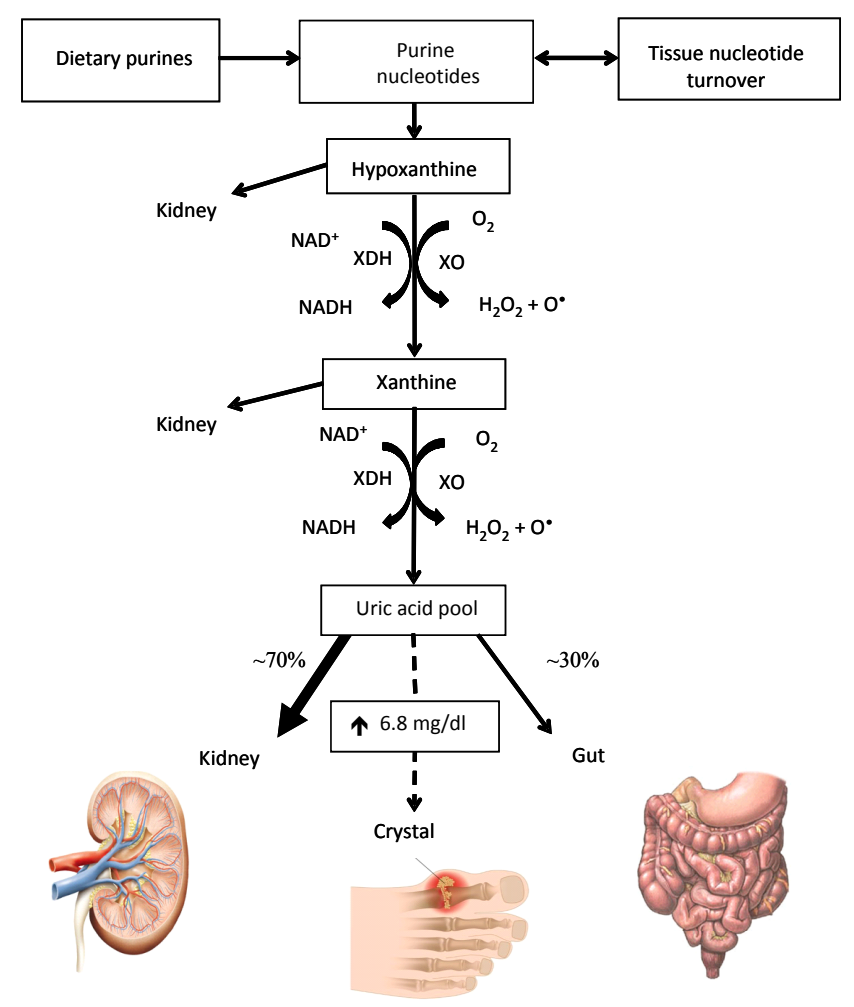

Figure 1. Purine metabolism

In healthy individuals, serum uric acid is maintained within the normal range by a complex system of secretion, reabsorption, and excretion via the kidneys or intestines. Under normal conditions, about $90 \%$ of the uric acid filtered is actively reabsorbed in the renal proximal tubules. The other $10 \%$ is for two-third excreted via the kidneys, and the remaining third via the 'extra-renal' pathway (gastrointestinal tract). ${ }^{8}$ Several transporters involved in uric acid secretion and reabsorption are known. In recent years, genome-wide association studies found several novel loci that were associated with serum uric acid concentrations, including SLC22A12 (URAT1), SLC2A (GLUT9), SLC17A3 (NPT4), and $A B C G 2(0141 \mathrm{~K})$ etc. ${ }^{16-18}$ These loci may indicate proteins involved in the regulation of uric acid excretion, but functional data are not yet available for all of these loci.

An unbalance, either in uric acid overproduction, underexcretion or both can cause hyperuricemia. Generally hyperuricemia is defined as a serum uric acid concentration 
greater than the solubility of uric acid $(408 \mu \mathrm{mol} / \mathrm{L}) .{ }^{19}$ In approximately $90 \%$ of the cases hyperuricemia is caused by underexcretion of uric acid, as a result of reduced renal function or the use of diuretics. In the other cases, overproduction of uric acid might cause hyperuricemia. This can be due to conditions with a high cell turnover such as tumour lysis syndrome or a genetic disorder of enzymes involved in the purine metabolism. For example, a deficiency in hypoxanthine-guanine phosphoribosyl transferase (HGPRT), leads to accumulation of hypoxanthine and guanine, and causes elevated serum uric acid concentrations. ${ }^{20}$ Next to this, consumption of diets containing large amount of purines (e.g. organ meat, seafood, beer), or intake of beverages sweetened by fructose whose digestion leads to ATP depletion and consequently the generation of adenosine monophosphate (AMP), may also increase serum uric acid concentrations. $^{21}$

\section{Uric acid and blood pressure}

Hypertension, a lasting increase in blood pressure, is the most potent risk factor for cardiovascular disease. Globally, approximately $25-35 \%$ of the adult population and more than $60 \%$ of the individuals over the age of 65 years have high blood pressure. ${ }^{22}$ Since the 1980s uric acid has been suspected to be a risk factor for hypertension. ${ }^{23}$ It is proposed that uric acid induces hypertension via several mechanisms.

One of the underlying mechanisms is the activation of the renin-angiotensin system, leading to increased production of the vasoconstrictor angiotensin $11 .{ }^{24}$ Hereby, uric acid affects directly the blood vessel and causes a reversible increase in blood pressure. Prolonged elevation of uric acid leads to the second and irreversible phase of hypertension (Figure 2). ${ }^{25}$ One pathway involves the uptake of uric acid by vascular smooth muscle cells (VSMCs), which causes proliferation and arteriolosclerosis that impairs pressure natriuresis, causing sodium-sensitive hypertension. Also, inflammation has been put forward as a possible underlying mechanism. ${ }^{26-28}$ The crystallisation of monosodium urate in joints or other tissues triggers the inflammasome (e.g. cryopyrin/ NLRP3) to generate the proinflammatory cytokine IL-1. ${ }^{29,30}$

Next to experimental studies, the association between uric acid and blood pressure has been extensively investigated in an epidemiological manner. Recent meta-analyses showed a significant association between serum uric acid and incident hypertension, independent of traditional risk factors. ${ }^{12,31}$ However, the association weakened in more recent studies. ${ }^{20}$ The later studies tended to adjust for more covariates, and some of these covariates might have been at the causal pathway of interest. Whether there is overadjustment or uric acid should indeed be considered as an innocent bystander instead of a true risk factor is still undecided.

More conclusive evidence can be provided by the use of a Mendelian randomization approach, which accounts for unmeasured confounding and for reverse causation by using genetic variants as proxies for the exposure. ${ }^{32}$ Studies using a Mendelian randomization approach, by using genetic markers associated with serum uric acid 
$14 \mid$ Chapter 1

concentration (e.g. SLC2A9, a uric acid transporter), have not been able to convincingly demonstrate that the association between uric acid and blood pressure is causal. ${ }^{16,33-39}$ The findings from these studies were generally interpreted as lack of a causal association between uric acid and blood pressure. However, polymorphisms in uric acid excretion genes only explain variation in serum uric acid concentration, but not in uric acid production, which we hypothesize to be a relevant factor for the development of hypertension.

Phase 1: Reversible Vasoconstriction

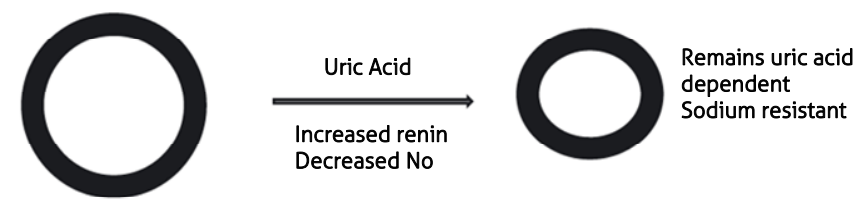

Phase 2: Arteriolar Wall Thickening
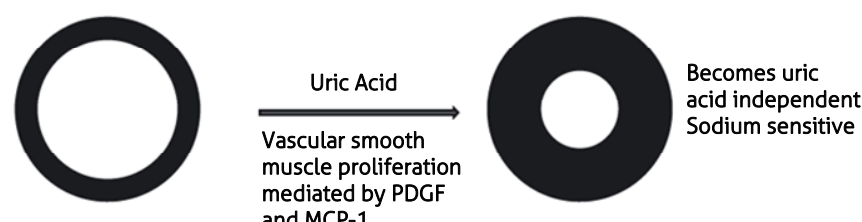

Figure 2. Two phases how uric acid may influence blood presure ${ }^{25}$

\section{The role of uric acid production}

In the two terminal steps of uric acid production, XOR is the sole enzyme responsible for the oxidation of hypoxanthine to xanthine, and the latter to uric acid. XOR exists in two forms, xanthine dehydrogenase $(X D H)$ and xanthine oxidase $(X O)$, which both occur in vivo. Initially, the XOR enzyme exists in XDH form, but when released into the circulation it is converted into $\mathrm{XO}$ and circulates to remote sites where it binds to the surface of endothelial cells. ${ }^{15,40-45}$ Although $\mathrm{XDH}$ preferentially reduces nicotinamide adenine dinucleotide $(\mathrm{NAD}+)$, both forms of the enzyme can also reduce molecular oxygen to form the reactive oxygen species (ROS) superoxide and hydrogen peroxide. Thus, during the production of uric acid, ROS are formed which may cause oxidative stress. Furthermore, the generated superoxide increases the formation of peroxynitrite, leading to an increase in endothelial NO synthase (eNOS) uncoupling resulting in even more ROS formation. Next to this, angiotensin II substantially increases endothelial $X O$ activation in cultured endothelial cells, subsequent to $\mathrm{NAD}(\mathrm{P}) \mathrm{H}$ oxidase activation, leading to even more ROS formation and inactivation of the vasodilator NO. ${ }^{46}$ Through these mechanisms, XOR is believed to be involved in the pathogenesis of ischemia-reperfusion injury. Elevated concentrations of circulating XOR have been found in several conditions, 
including atherosclerosis, ${ }^{15}$ suggesting that indeed the increased production of uric acid and the concomitant formation of ROS may be involved in the aetiology of hypertension and cardiovascular diseases.

\section{Proxies for uric acid production}

Since the production of uric acid may contribute, independent of uric acid concentration, to the pathogenesis of hypertension, the production should be investigated as well. Facing the problem that it is not possible to directly measure uric acid production in a large population of individuals and the concentration of uric acid in serum is not an adequate marker for production, proxies for uric acid production need to be investigated. In this thesis we studied three proxies for uric acid production: (i) variants of the XOR gene, (ii) different ratios of the purine metabolites, and (iii) 24-h urinary uric acid excretion.

\section{(i) Genetic variants of the xanthine oxidoreductase gene}

The human XOR gene, encoding the XOR enzyme, has been mapped to chromosome 2 p22. ${ }^{47-49}$ The activity of the enzyme is the highest in liver ${ }^{50-52}$ and intestine ${ }^{53}$, whereas most other tissues show little activity. ${ }^{54-56}$ Two in-vitro studies addressed the impact of variation in the XOR gene on XOR activity. ${ }^{57,58}$ Molecular studies of the DNA of 96 Japanese individuals, revealed three nonsynonymous single nucleotide polymorphisms (SNPs). Functional characterization of 21 XOR variants revealed deficient enzyme activity in two, and reduced and enhanced activity in six and two, respectively. ${ }^{57}$ Among 13 SNPs in the XOR 5'-flanking region, one was associated with decreased expression in transfected cells. ${ }^{58}$ The association between XOR expression and variation in the gene offers the opportunity to explore the role of $X O R$ as a hypertension susceptibility gene by using SNPs in the XOR gene as a proxy for uric acid production.

\section{(ii) Ratios of purine metabolites}

In the terminal steps of purine catabolism, the rate-limiting enzyme XOR catalyses the oxidation of hypoxanthine to xanthine and xanthine to uric acid. An increased XOR activity, resulting in increased uric acid production, may lead to a relative decrease in the more upstream metabolites, thus, lower hypoxanthine compared to xanthine and lower xanthine compared to uric acid concentration, resulting in higher ratios of xanthine/hypoxanthine, uric acid/xanthine, and uric acid/hypoxanthine. The latter is based on animal studies and studies examining patients with XOR deficiency, a genetic disorder called xanthinuria, who have decreased concentrations of the more upstream metabolites. $^{59-62}$ 
$16 \mid$ Chapter 1

(iii) Uric acid excretion in urine

Uric acid is produced during the metabolism of endogenous and exogenous purines. Once produced, uric acid cannot be further metabolized and needs to be eliminated by either renal or extra-renal excretion, so that serum uric acid remains stable and within the normal range of $200-408 \mu \mathrm{mol} / \mathrm{L}$ for men and $120-340 \mu \mathrm{mol} / \mathrm{L}$ for women. In the clinic gout patients with hyperuricemia are classified as overproducers or underexcretors based on the amount of uric acid excreted in the urine in 24 hours. ${ }^{63}$ According to this criterion, approximately $10 \%$ of patients with hyperuricemia are overproducers. ${ }^{8}$ Overproduction may however have implications beyond individuals with hyperuricemia and can also occur in patients with normal serum uric acid concentration. If their excretion capacity is adequate, they will not develop hyperuricemia, but may still suffer from the adverse consequences of increased uric acid production. Previous studies have shown that an increase in uric acid production, either by an increased endogenous or exogenous supply, increases urinary uric acid excretion. ${ }^{64,65}$ Since uric acid is predominantly excreted via the kidneys, we studied 24-h urinary uric acid excretion as a proxy for uric acid production.

\section{Blood pressure components}

Several components can be useful in examining the association between uric acid and blood pressure, including diastolic and systolic blood pressure, mean arterial pressure and pulse pressure. Each component might reflect a different pathophysiological state and the relative importance of the component in predicting cardiovascular risk differs according to age (Table 1$)$.

In younger individuals ( $<50$ years of age), diastolic and to a lesser extent systolic blood pressure are the main predictors of cardiovascular risk. After the age of 50 pulse pressure supersedes these to become the main predictor of cardiovascular risk. ${ }^{66-68}$ Franklin et al, showed that in these older individuals combining pulse pressure and mean arterial pressure is superior to any single blood pressure component for predicting cardiovascular risk. ${ }^{69}$ Moreover, pulse pressure and mean arterial pressure may also provide greater insight into the different arterial stiffness and peripheral resistance, respectively. ${ }^{69}$

The steady component of blood pressure, captured by mean arterial pressure, reflects peripheral vascular resistance. As previously described, there are several plausible mechanisms how uric acid might increase vascular resistance, for example via accumulation of ROS. ${ }^{24}$ Prolonged exposure to increased peripheral vascular resistance and high blood pressure leads to embedding of the vessel structure in a remodelled extracellular matrix. ${ }^{70,71}$ Initially, the remodelling of arteries will be adaptive, but it eventually becomes maladaptive and increases stiffness, contributing to cardiovascular complications of hypertension. ${ }^{70}$ This is reflected in the pulsatile blood components, captured by pulse pressure. 
\begin{tabular}{l|l} 
& \\
General introduction &
\end{tabular}

Table 1. Simplified overview of the characteristics of the different blood pressure components

\begin{tabular}{|c|c|c|c|c|}
\hline Component & Causes for elevation & Reflecting & Calculation & $\begin{array}{l}\text { Indicator for } \\
\text { cardiovascular } \\
\text { risk }\end{array}$ \\
\hline $\begin{array}{l}\text { Systolic blood } \\
\text { pressure }\end{array}$ & $\begin{array}{l}\text { Stiffening of the arteries, due to high } \\
\text { blood pressure or fatty deposits } \\
\text { damaging the walls (atherosclerosis) }\end{array}$ & $\begin{array}{l}\text { Main determined } \\
\text { of pulsatile } \\
\text { component }\end{array}$ & $\begin{array}{l}\text { Maximum pressure } \\
\text { during one } \\
\text { heartbeat }\end{array}$ & $\begin{array}{l}\text { Young, }<50 \text { years } \\
\text { of age }\end{array}$ \\
\hline $\begin{array}{l}\text { Diastolic } \\
\text { blood } \\
\text { pressure }\end{array}$ & $\begin{array}{l}\text { Increased peripheral resistance, due } \\
\text { to vasoconstriction }\end{array}$ & $\begin{array}{l}\text { Main determined } \\
\text { of steady } \\
\text { component }\end{array}$ & $\begin{array}{l}\text { Minimum pressure } \\
\text { in between two } \\
\text { heart beats }\end{array}$ & $\begin{array}{l}\text { Young, }<50 \text { years } \\
\text { of age }\end{array}$ \\
\hline Pulse pressure & $\begin{array}{l}\text { Stiffening of the arteries, due to high } \\
\text { blood pressure or fatty deposits } \\
\text { damaging the walls (atherosclerosis) }\end{array}$ & $\begin{array}{l}\text { Pulsatile } \\
\text { component }\end{array}$ & $\begin{array}{l}\text { Systolic minus } \\
\text { diastolic blood } \\
\text { pressure }\end{array}$ & $\begin{array}{l}\text { Old, }>50 \text { years of } \\
\text { age }\end{array}$ \\
\hline $\begin{array}{l}\text { Mean arterial } \\
\text { pressure }\end{array}$ & $\begin{array}{l}\text { Increased peripheral resistance, due } \\
\text { to vasoconstriction }\end{array}$ & $\begin{array}{l}\text { Steady } \\
\text { component }\end{array}$ & $\begin{array}{l}\text { Diastolic blood } \\
\text { pressure plus } 1 / 3 \text { of } \\
\text { pulse pressure }\end{array}$ & $\begin{array}{l}\text { Old, }>50 \text { years of } \\
\text { age }\end{array}$ \\
\hline
\end{tabular}

\section{Gout management}

Gout is the most common form of inflammatory arthritis, caused by formation of monosodium urate crystals in and around peripheral joints as a consequence of persistent hyperuricemia. The prevalence varies from $1.4 \%{ }^{72}$ in Europe to $3.9 \%{ }^{9}$ in the United States and several studies showed an increase in incidence and prevalence. For example, the prevalence increased by $63.9 \%$ and the incidence increased by $29.6 \%$ between 1997 and 2012 in the UK. ${ }^{73}$

Gout is considered a well-treatable disease: the inflammation of acute attacks can be treated by nonsteroidal anti-inflammatory drugs (NSAIDS), colchicine or corticosteroids, and in case of recurrent attacks or tophaceous gout reduction of serum uric acid is recommended. The most used uric acid lowering agents are the XOR inhibitors allopurinol and febuxostat. Despite our understanding of the pathophysiology and availability of effective treatment, the management of gout is considered as suboptimal. ${ }^{7,74}$ Given the rising burden of gout on the population ${ }^{73}$ and its associated impact on patients, insights into the barriers of gout management are required. Common barriers for effective management can be distinguished between patients and physician barriers and, as it is likely that both play a significant role, both should be addressed. ${ }^{6}$

\section{Patients medication adherence}

Poor medication adherence to long-term uric acid lowering therapy may be an important contributor to suboptimal outcomes seen in gout. Patients not taking their medication risk having a gout flare and the chance of obtaining joint damage will become higher. ${ }^{75}$ Moreover, the healthcare provider might unnecessarily increase the dose of uric acid lowering therapy or prematurely switch to other, more expensive therapies, like benzbromarone. As in other chronic conditions, medication adherence tends to be poor, because patients might not feel ill, do not experience the effect of the medication but 
$18 \mid$ Chapter 1

only the side-effects, or are more afraid for the side effects of the medication than for the disease. Across seven common chronic conditions including hypertension, osteoporosis, and diabetes, patients suffering from gout had the poorest medication adherence. $^{76}$ A systematic overview of available literature addressing the problem of poor medication adherence among gout patients is needed.

\section{Definition and measurement of medication adherence}

According to the World Health Organization adherence is defined as 'the extent to which a person's behaviour - taking medication, following a diet, and/or executing lifestyle changes, corresponds with agreed recommendations from a healthcare provider. ${ }^{.77}$ There is no "gold standard" for measuring adherence. ${ }^{78}$ Medication adherence can be measured either directly, for example by measuring drug metabolites or drug levels in blood, urine or tissues, or indirectly, by self-report, electronic monitoring, pharmacy records or healthcare provider assessment. Independently of the measurement technique used, the threshold for defining "good" and "poor" medication adherence is arbitrary. ${ }^{78}$

To characterize medication adherence different concepts should be studied. According to the new taxonomy from 2012 it includes initiation, implementation, non-persistence, and overall adherence during observation time (Figure 3). ${ }^{79}$ Poor medication adherence can thus occur in the following situations or combinations thereof: late or non-initiation of the prescribed treatment, sub-optimal implementation of the dosing regimen, or early discontinuation of the treatment. ${ }^{79}$

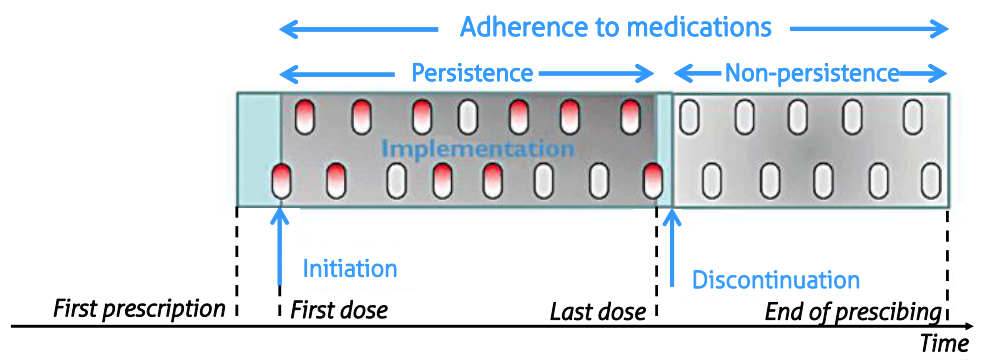

Figure 3. Illustration of the process of adherence to medication ${ }^{79}$

Thus far, the available literature addresses mostly the problem of adherence during the entire observation time, but little is known on the dynamics of medication use among gout patients. While non-persistence, the occurrence of a gap in therapy, may have little clinical effect in certain chronic diseases, an interruption in uric acid lowering therapy might actually trigger or prolong gouty attacks. ${ }^{80}$ Furthermore, it is unknown whether those experiencing a gap return to therapy and if medication adherence improved after restarting therapy. Further research is therefore required to obtain insights into dynamic patterns of medication adherence and to identify patients at high risk of becoming nonadherent and non-persistent. Several factors such as older age, suffering from certain 
comorbidities like hypertension and diabetes, and the use of anti-hypertensive medication were associated with higher adherence rates, but these factors have not or poorly (e.g. age) been studied in relation to persistence.

\section{Gout management by the general practitioner}

General practitioners are the most relevant healthcare professional when it comes to the diagnosis and treatment of gout. Several societies published recommendations for the management of gout, including the American College of Rheumatology (ACR) and the European League Against Rheumatism (EULAR). ${ }^{81-83}$ Despite these recommendations, the management of gout is considered suboptimal. ${ }^{84-86} \mathrm{~A}$ study conducted in the UK among primary care physicians showed that lifestyle advice was infrequently offered and allopurinol was only prescribed to a minority. ${ }^{86}$ Appropriate initiation and dosing of uric acid lowering therapy are the main factors to achieve the therapeutic target level for serum uric acid. ${ }^{87}$ Whenever this fails, patients are at increased risk for having more gout flares and for developing tophi and subsequent joint damage. Furthermore, a more severe and untreated disease will in the long run lead to increased gout-related healthcare costs. ${ }^{88,89}$

Clinical practice behaviour is influenced by several aspects, including gout knowledge and illness perceptions. Up to now only a few small qualitative studies have been performed to elucidate barriers from the general practitioner side. They showed a moderate knowledge of the causes and consequences of gout. ${ }^{6,90}$ Some general practitioners revealed that they still had a negative stereotype view and perceived stigma that the disease is self-inflicted and only a consequence of an unhealthy lifestyle. ${ }^{6}$ In some cases gout was still considered as an acute disease rather than a chronic disease, and the long-term management could therefore be inadequate. ${ }^{6,91}$ Although it is recommended to prescribe uric acid lowering therapy in patients with recurrent flares or tophi, this is only done for a small percentage of patients. The same applies to measurement of serum uric acid concentrations on a regular basis. ${ }^{73,91}$

Taken together, knowledge, illness perceptions, and clinical behaviour of the general practitioner are important aspects to achieve optimal gout management. Because of the complex interactions between these aspects, studies addressing all these aspects at the same time are essential when aiming to improve gout management. However, this has not been done yet.

\section{Main aims}

The research presented in this thesis was conducted in several population-based cohort studies and made use of general practitioner interviews. The aim of this thesis was twofold:

(i) to investigate the role of uric acid production in the association between uric acid and blood pressure; and 
$20 \mid$ Chapter 1

(ii) to explore medication adherence among gout patients and gout management by the general practitioner.

\section{OUTLINE OF THIS THESIS}

This thesis is divided into two parts. In Part I we examined the association between uric acid and blood pressure, in particular the role of uric acid production. In Part II of this thesis we studied medication adherence among gout patients and gout management of general practitioners.

\section{Part I Uric acid and blood pressure: the role of uric acid production}

In chapter 2, we examined associations of genetic variation in the XOR gene, reflecting uric acid production, with secular trends in the steady and pulsatile blood pressure components and the risk of hypertension. The study was conducted in the Flemish Study on Environment, Genes and Health Outcomes (FLEMENGHO) and the European Project on Genes in Hypertension (EPOGH). The FLEMENGHO study is a prospective populationbased cohort study. ${ }^{92}$ From August 1985 to November 1990 participants were recruited in the geographically defined area in Northern Belgium. The EPOGH project recruited participants from 1999 to 2001 in four centres: Pilsen (Czech Republic), Mirano (Italy), Kraków (Poland), and Novosibirsk (Russian Federation). ${ }^{93}$ In EPOGH, one follow-up examination took place from 2006 to 2008 and in FLEMENGHO follow-up lasted until 2013. In total, data from 4535 individuals who participated in FLEMENGHO or the EPOGH project were available. Data of 2769 participants with a blood pressure measurement and genotype at XOR was used.

In chapter 3, we examined associations of purine metabolite ratios, considered as proxies for an increased XOR activity, with blood pressure and extended existing evidence on the association of plasma uric acid with blood pressure to school-age children. The study was conducted in the Dutch KOALA Birth Cohort Study, an acronym (in Dutch) for: Child, parents and health: lifestyle and genetic constitution. From October 2000 until December 2002, a total of 2834 pregnant women were recruited at 34 weeks of gestation. A subgroup of 1204 parents was asked for consent for a home visit for anthropometric measurements, collection of venous blood, and a blood pressure measurement from their child at the age of 6-7 years. Data of 246 children with a blood pressure measurement and a venous blood collection was used.

In chapter 4, we examined associations of serum uric acid and uric acid production, captured by urinary uric acid excretion, with the steady and pulsatile blood pressure components and prevalent hypertension. This study was conducted in The Maastricht Study, an observational cohort study. All individuals aged between 40 and 75 years and living in the southern part of The Netherlands were eligible for participation. The study focuses on the aetiology, pathophysiology, complications, and comorbidities of type 2 
diabetes mellitus and is characterized by an extensive phenotyping approach. For reasons of efficiency, the study population was enriched with type 2 diabetes mellitus patients. In total, 3451 participants completed the baseline survey between November 2010 and September 2013. Cross-sectional data was used from a subset of 2555 participants from whom a 24-h blood pressure measurement, 24-h urine collection and venous blood samples were obtained.

\section{Part II Gout management by the general practitioner and patient}

In chapter 5, we assessed medication adherence among gout patients treated with allopurinol. This study was conducted in the Clinical Practice Research Datalink (CPRD) from the UK, formerly known as the General Practitioner Database. The database comprises prospectively collected computerized medical records from general practitioners. Since 1987, data are prospectively recorded, and include patient demographics, prescription details, clinical events, laboratory test results, specialist referrals, and hospitalizations. Data of over 11.3 million patients from 674 practices from the UK is available. ${ }^{94}$ For this study, newly diagnosed gout patients initiated with allopurinol in the period of valid data collection ( $1^{\text {st }}$ January 1987 through $30^{\text {th }}$ of June 2014), were included. Longitudinal data of 48,834 newly diagnosed gout patients initiated allopurinol were used to assess medication adherence, including nonadherence, non-persistence, and those who return to therapy after the occurrence of a gap in therapy and their subsequent adherence to medication. Additionally, the association of various factors with medication adherence was evaluated.

In chapter 6, we performed a systematic literature review and meta-analysis on medication adherence among gout patients. Furthermore, we investigated which factors were associated with medication adherence.

In chapter 7, we explored knowledge, illness perceptions, and stated practice behaviour in relation to gout in primary care. For this study we collected data by interviewing 32 general practitioners from the southern part of The Netherlands. A mixed-methods approach, using questionnaires and structured interviews, was used.

In chapter 8, we discuss the main results and methodologic issues of the studies described in the earlier chapters, and implications for further research and clinical practice are given. 
$22 \mid$ Chapter 1

\section{REFERENCES}

1. Kuo CF, Grainge MJ, Zhang W, et al. Global epidemiology of gout: prevalence, incidence and risk factors. Nat Rev Rheumatol. 2015; 11(11):649-662.

2. Schumacher HR, Jr., Becker MA, Wortmann RL, et al. Effects of febuxostat versus allopurinol and placebo in reducing serum urate in subjects with hyperuricemia and gout: a 28-week, phase III, randomized, double-blind, parallel-group trial. Arthritis Rheum. 2008; 59(11):1540-1548.

3. Eason A, House ME, Vincent $Z$, et al. Factors associated with change in radiographic damage scores in gout: a prospective observational study. Ann Rheum Dis. 2016; 75(12):2075-2079.

4. Schlesinger N, Thiele RG. The pathogenesis of bone erosions in gouty arthritis. Ann Rheum Dis. 2010; 69(11): 1907-1912.

5. Wertheimer A, Morlock R, Becker MA. A revised estimate of the burden of illness of gout. Curr Ther Res Clin Exp. 2013; 75:1-4.

6. Spencer K, Carr A, Doherty M. Patient and provider barriers to effective management of gout in general practice: a qualitative study. Ann Rheum Dis. 2012; 71(9):1490-1495.

7. Doherty $M$, Jansen $T L$, Nuki G, et al. Gout: why is this curable disease so seldom cured? Ann Rheum Dis. 2012; 71(11):1765-1770.

8. Richette P, Bardin T. Gout. Lancet. 2010; 375(9711):318-328.

9. Zhu Y, Pandya BJ, Choi HK. Prevalence of gout and hyperuricemia in the US general population: the National Health and Nutrition Examination Survey 2007-2008. Arthritis Rheum. 2011; 63(10):3136-3141.

10. Niskanen LK, Laaksonen DE, Nyyssonen K, et al. Uric acid level as a risk factor for cardiovascular and all-cause mortality in middle-aged men: a prospective cohort study. Arch Intern Med. 2004; 164(14): 1546-1551.

11. Feig DI, Kang DH, Johnson RJ. Uric acid and cardiovascular risk. N Engl J Med. 2008; 359(17):1811-1821.

12. Grayson PC, Kim SY, LaValley $M$, et al. Hyperuricemia and incident hypertension: a systematic review and meta-analysis. Arthritis Care Res (Hoboken). 2011; 63(1): 102-110.

13. Mazzali M, Hughes ], Kim YG, et al. Elevated uric acid increases blood pressure in the rat by a novel crystal-independent mechanism. Hypertension. 2001; 38(5):1101-1106.

14. Mazzali M, Kanellis J, Han L, et al. Hyperuricemia induces a primary renal arteriolopathy in rats by a blood pressure-independent mechanism. Am J Physiol Renal Physiol. 2002; 282(6):F991-997.

15. Harrison R. Structure and function of xanthine oxidoreductase: where are we now? Free Radic Biol Med. 2002; 33(6):774-797.

16. Vitart V, Rudan I, Hayward C, et al. SLC2A9 is a newly identified urate transporter influencing serum urate concentration, urate excretion and gout. Nat Genet. 2008; 40(4):437-442.

17. Enomoto A, Kimura $H$, Chairoungdua $A$, et al. Molecular identification of a renal urate anion exchanger that regulates blood urate levels. Nature. 2002; 417(6887):447-452.

18. Kolz M, Johnson T, Sanna $S$, et al. Meta-analysis of 28,141 individuals identifies common variants within five new loci that influence uric acid concentrations. PLOS Genet. 2009; 5(6):e1000504.

19. Fiddis RW, Vlachos N, Calvert PD. Studies of urate crystallisation in relation to gout. Ann Rheum Dis. 1983; 42 Suppl 1:12-15.

20. Kelley WN, Rosenbloom FM, Henderson JF, et al. A specific enzyme defect in gout associated with overproduction of uric acid. Proc Natl Acad Sci U S A. 1967; 57(6):1735-1739.

21. Beyl RN, Jr., Hughes L, Morgan S. Update on Importance of Diet in Gout. Am J Med. 2016; 129(11):1153-1158.

22. Wolf-Maier K, Cooper RS, Banegas JR, et al. Hypertension prevalence and blood pressure levels in 6 European countries, Canada, and the United States. JAMA. 2003; 289(18):2363-2369.

23. Messerli FH, Frohlich ED, Dreslinski GR, et al. Serum uric acid in essential hypertension: an indicator of renal vascular involvement. Ann Intern Med. 1980;93(6):817-821.

24. Yu MA, Sanchez-Lozada LG, Johnson RJ, et al. Oxidative stress with an activation of the reninangiotensin system in human vascular endothelial cells as a novel mechanism of uric acid-induced endothelial dysfunction. J Hypertens. 2010; 28(6):1234-1242.

25. Feig DI. The role of uric acid in the pathogenesis of hypertension in the young. J Clin Hypertens (Greenwich). 2012; 14(6):346-352.

26. Dalekos GN, Elisaf M, Bairaktari $E$, et al. Increased serum levels of interleukin-1beta in the systemic circulation of patients with essential hypertension: additional risk factor for atherogenesis in hypertensive patients? J Lab Clin Med. 1997; 129(3):300-308. 
General introduction $\quad 23$

27. Dalekos GN, Elisaf MS, Papagalanis $N$, et al. Elevated interleukin-1 beta in the circulation of patients with essential hypertension before any drug therapy: a pilot study. Eur J Clin Invest. 1996; 26(10):936-939.

28. Peeters $A C$, Netea MG, Janssen MC, et al. Proinflammatory cytokines in patients with essential hypertension. Eur J Clin Invest. 2001; 31(1):31-36.

29. Rock KL, Kataoka H, Lai J]. Uric acid as a danger signal in gout and its comorbidities. Nat Rev Rheumatol. 2013; 9(1):13-23.

30. Zhou R, Tardivel A, Thorens B, et al. Thioredoxininteracting protein links oxidative stress to inflammasome activation. Nat Immunol. 2010; 11(2):136-140.

31. Wang J, Oin T, Chen J, et al. Hyperuricemia and risk of incident hypertension: a systematic review and meta-analysis of observational studies. PLoS One. 2014; 9(12):e114259.

32. Sheehan NA, Didelez V, Burton PR, et al. Mendelian randomisation and causal inference in observational epidemiology. Plos Med. 2008; 5(8):e177.

33. Caulfield MJ, Munroe PB, O'Neill D, et al. SLC2A9 is a high-capacity urate transporter in humans. Plos Med. 2008; 5(10):1509-1523.

34. Han X, Gui L, Liu B, et al. Associations of the uric acid related genetic variants in SLC2A9 and ABCG2 loci with coronary heart disease risk. BMC Genet. 2015; 16:1-7.

35. Mallamaci F, Testa A, Leonardis $D$, et al. A polymorphism in the major gene regulating serum uric acid associates with clinic SBP and the white-coat effect in a family-based study. J Hypertens. 2014; 32(8):1621-1628; discussion 1628.

36. Mallamaci F, Testa A, Leonardis $D$, et al. A genetic marker of uric acid level, carotid atherosclerosis, and arterial stiffness: a familybased study. Am J Kidney Dis. 2015; 65(2):294302.

37. Palmer TM, Nordestgaard BG, Benn $M$, et al. Association of plasma uric acid with ischaemic heart disease and blood pressure: mendelian randomisation analysis of two large cohorts. Bmj. 2013; 347:f4262.

38. Parsa A, Brown E, Weir MR, et al. Genotypebased changes in serum uric acid affect blood pressure. Kidney Int. 2012; 81(5):502-507.

39. Yang $\mathrm{O}$, Kottgen A, Dehghan A, et al. Multiple genetic loci influence serum urate levels and their relationship with gout and cardiovascular disease risk factors. Circ Cardiovasc Genet. 2010; 3(6): 523-530.
40. Houston M, Estevez A, Chumley P, et al. Binding of xanthine oxidase to vascular endothelium. Kinetic characterization and oxidative impairment of nitric oxide-dependent signaling. J Biol Chem. 1999; 274(8):4985-4994.

41. Adachi T, Fukushima T, Usami Y, et al. Binding of human xanthine oxidase to sulphated glycosaminoglycans on the endothelial-cell surface. Biochem J. 1993; 289 ( Pt 2):523-527.

42. Radi R, Rubbo H, Bush K, et al. Xanthine oxidase binding to glycosaminoglycans: kinetics and superoxide dismutase interactions of immobilized xanthine oxidase-heparin complexes. Arch Biochem Biophys. 1997; 339(1):125-135

43. Rouquette $M$, Page $S$, Bryant $R$, et al. Xanthine oxidoreductase is asymmetrically localised on the outer surface of human endothelial and epithelial cells in culture. FEBS Lett. 1998; 426(3):397-401.

44. Vickers S, Schiller HJ, Hildreth JE, et al. Immunoaffinity localization of the enzyme xanthine oxidase on the outside surface of the endothelial cell plasma membrane. Surgery. 1998; 124(3):551-560.

45. Pritsos CA. Cellular distribution, metabolism and regulation of the xanthine oxidoreductase enzyme system. Chem Biol Interact. 2000; 129(12):195-208.

46. Landmesser U, Spiekermann S, Preuss C, et al. Angiotensin $\|$ induces endothelial xanthine oxidase activation: role for endothelial dysfunction in patients with coronary disease. Arterioscler Thromb Vasc Biol. 2007; 27(4):943948.

47. Xu P, Zhu XL, Huecksteadt TP, et al. Assignment of human xanthine dehydrogenase gene to chromosome 2p22. Genomics. 1994; 23(1): 289291.

48. Ichida K, Amaya Y, Noda K, et al. Cloning of the cDNA encoding human xanthine dehydrogenase (oxidase): structural analysis of the protein and chromosomal location of the gene. Gene. 1993; 133(2): 279-284.

49. Saksela M, Raivio KO. Cloning and expression in vitro of human xanthine dehydrogenase/ oxidase. Biochem J. 1996; 315 ( Pt 1):235-239.

50. Della Corte E, Gozzetti G, Novello F, et al. Properties of the xanthine oxidase from human liver. Biochim Biophys Acta. 1969; 191(1):164166.

51. Guerciolini R, Szumlanski C, Weinshilboum RM. Human liver xanthine oxidase: nature and extent of individual variation. Clin Pharmacol Ther. 1991; 50(6): 663-672. 
24 Chapter 1

52. Stirpe F, Ravaioli M, Battelli MG, et al. Xanthine oxidoreductase activity in human liver disease. Am J Gastroenterol. 2002; 97(8):2079-2085.

53. Auscher C, Amory N, van der Kemp P, et al. Xanthine oxidase activity in human intestines. Histochemical and radiochemical study. Adv Exp Med Biol. 1979; 122B:197-201.

54. Parks DA, Granger DN. Xanthine oxidase: biochemistry, distribution and physiology. Acta Physiol Scand Suppl. 1986; 548:87-99.

55. Sarnesto A, Linder N, Raivio KO. Organ distribution and molecular forms of human xanthine dehydrogenase/xanthine oxidase protein. Lab Invest. 1996; 74(1): 48-56.

56. Saksela M, Lapatto R, Raivio KO. Xanthine oxidoreductase gene expression and enzyme activity in developing human tissues. Biol Neonate. 1998; 74(4):274-280.

57. Kudo M, Moteki T, Sasaki T, et al. Functional characterization of human xanthine oxidase allelic variants. Pharmacogenet Genomics. 2008; 18(3): 243-251.

58. Kudo M, Sasaki T, Ishikawa M, et al. Functional characterization of genetic polymorphisms identified in the promoter region of the xanthine oxidase gene. Drug Metab Pharmacokinet. 2010; 25(6):599-604.

59. Chen O, Park HC, Goligorsky MS, et al. Untargeted plasma metabolite profiling reveals the broad systemic consequences of xanthine oxidoreductase inactivation in mice. PLoS One. 2012; 7(6): e37149.

60. Tanaka K, Kanazawa I, Yamasaki H, et al. Xanthinuria Type I With a Novel Mutation of Xanthine Dehydrogenase. Am J Med Sci. 2015; 350(2):155-156.

61. Zhou $Y N$, Zhang $X G$, Ding $R$, et al. Using NextGeneration Sequencing to Identify a Mutation in Human MCSU that is Responsible for Type II Xanthinuria. Cell Physiol Biochem. 2015; 35(6): 2412-2421.

62. Curto R, Voit EO, Sorribas A, et al. Mathematical models of purine metabolism in man. Math Biosci. 1998; 151(1):1-49.

63. Ichida K, Matsuo H, Takada T, et al. Decreased extra-renal urate excretion is a common cause of hyperuricemia. Nat Commun. 2012; 3:764.

64. Puig JG, Torres RJ, de Miguel E, et al. Uric acid excretion in healthy subjects: a nomogram to assess the mechanisms underlying purine metabolic disorders. Metabolism. 2012; 61(4):512-518.

65. Loffler W, Grobner W, Medina R, et al. Influence of Dietary Purines on Pool Size, Turnover, and Excretion of Uric-Acid during Balance Conditions
- Isotope Studies Using N-15-Uric Acid. Res Exp Med. 1982; 181(2):113-123.

66. Franklin SS, Larson MC, Khan SA, et al. Does the relation of blood pressure to coronary heart disease risk change with aging? The Framingham Heart Study. Circulation. 2001; 103(9):12451249.

67. Li Y, Wei FF, Thijs $L$, et al. Ambulatory hypertension subtypes and 24-hour systolic and diastolic blood pressure as distinct outcome predictors in 8341 untreated people recruited from 12 populations. Circulation. 2014; 130(6):466-474.

68. Li Y, Wei FF, Wang $S$, et al. Cardiovascular risks associated with diastolic blood pressure and isolated diastolic hypertension. Curr Hypertens Rep. 2014; 16(11): 489.

69. Franklin SS, Lopez VA, Wong ND, et al. Single versus combined blood pressure components and risk for cardiovascular disease: the Framingham Heart Study. Circulation. 2009; 119(2):243-250.

70. Intengan HD, Schiffrin EL. Vascular remodeling in hypertension: roles of apoptosis, inflammation, and fibrosis. Hypertension. 2001; 38(3 Pt 2):581-587.

71. Mulvany MJ. Vascular remodelling of resistance vessels: can we define this? Cardiovasc Res. 1999; 41(1):9-13.

72. Annemans L, Spaepen E, Gaskin M, et al. Gout in the UK and Germany: prevalence, comorbidities and management in general practice 20002005. Ann Rheum Dis. 2008; 67(7):960-966.

73. Kuo CF, Grainge M], Mallen C, et al. Rising burden of gout in the UK but continuing suboptimal management: a nationwide population study. Ann Rheum Dis. 2015; 74(4):661-667.

74. Rothenbacher D, Primatesta P, Ferreira A, et al. Frequency and risk factors of gout flares in a large population-based cohort of incident gout. Rheumatology (Oxford). 2011; 50(5):973-981.

75. Kavoussi H, Rezaei M, Derakhshandeh K, et al. Clinical Features and Drug Characteristics of Patients with Generalized Fixed Drug Eruption in the West of Iran (2005-2014). Dermatol Res Pract. 2015; 2015:236703.

76. Briesacher BA, Andrade SE, Fouayzi $H$, et al. Comparison of drug adherence rates among patients with seven different medical conditions. Pharmacotherapy. 2008; 28(4):437443.

77. Sabaté E. Adherence to long-term therapies: evidence for action. World Health Organization; 2003. 
78. Osterberg $\mathrm{L}$, Blaschke $\mathrm{T}$. Adherence to medication. N Engl J Med. 2005;353(5): 487-497.

79. Vrijens B, De Geest S, Hughes DA, et al. A new taxonomy for describing and defining adherence to medications. Br J Clin Pharmacol. 2012; 73(5):691-705.

80. Park H, Rascati KL, Prasla K, et al. Evaluation of health care costs and utilization patterns for patients with gout. Clin Ther. 2012; 34(3):640652.

81. Richette P, Doherty M, Pascual E, et al. 2016 updated EULAR evidence-based recommendations for the management of gout. Ann Rheum Dis. 2017; 76(1):29-42

82. Khanna D, Fitzgerald JD, Khanna PP, et al. 2012 American College of Rheumatology guidelines for management of gout. Part 1: systematic nonpharmacologic and pharmacologic therapeutic approaches to hyperuricemia. Arthritis Care Res (Hoboken). 2012; 64(10):14311446.

83. Khanna D, Khanna PP, Fitzgerald JD, et al. 2012 American College of Rheumatology guidelines for management of gout. Part 2: therapy and antiinflammatory prophylaxis of acute gouty arthritis. Arthritis Care Res (Hoboken). 2012; 64(10): 1447-1461.

84. Mikuls TR, Farrar JT, Bilker WB, et al. Suboptimal physician adherence to quality indicators for the management of gout and asymptomatic hyperuricaemia: results from the UK General Practice Research Database (GPRD). Rheumatology (Oxford). 2005; 44(8):1038-1042.

85. Neogi T, Hunter DJ, Chaisson CE, et al. Frequency and predictors of inappropriate management of recurrent gout attacks in a longitudinal study. J Rheumatol. 2006; 33(1):104-109.
86. Roddy E, Zhang W, Doherty M. Concordance of the management of chronic gout in a UK primary-care population with the EULAR gout recommendations. Ann Rheum Dis. 2007; 66(10):1311-1315.

87. Dalbeth N, House ME, Horne A, et al. Prescription and dosing of urate-lowering therapy, rather than patient behaviours, are the key modifiable factors associated with targeting serum urate in gout. BMC Musculoskelet Disord. 2012; 13:174.

88. Halpern R, Mody RR, Fuldeore MJ, et al. Impact of noncompliance with urate-lowering drug on serum urate and gout-related healthcare costs: administrative claims analysis. Curr Med Res Opin. 2009; 25(7):1711-1719.

89. Wu EQ, Patel PA, Yu AP, et al. Disease-related and all-cause health care costs of elderly patients with gout. J Manag Care Pharm. 2008; 14(2):164-175.

90. Vaccher S, Kannangara DR, Baysari MT, et al. Barriers to Care in Gout: From Prescriber to Patient. J Rheumatol. 2016; 43(1):144-149.

91. Jeyaruban A, Larkins S, Soden M. Management of gout in general practice--a systematic review. Clin Rheumatol. 2015; 34(1):9-16.

92. Staessen JA, Wang JG, Brand E, et al. Effects of three candidate genes on prevalence and incidence of hypertension in a Caucasian population. J Hypertens. 2001; 19(8):1349-1358.

93. Kuznetsova T, Staessen JA, Kawecka-Jaszcz K, et al. Quality control of the blood pressure phenotype in the European Project on Genes in Hypertension. Blood Press Monit. 2002; 7(4):215224.

94. Herrett E, Gallagher AM, Bhaskaran K, et al. Data Resource Profile: Clinical Practice Research Datalink (CPRD). Int J Epidemiol. 2015; 44(3): 827-836 



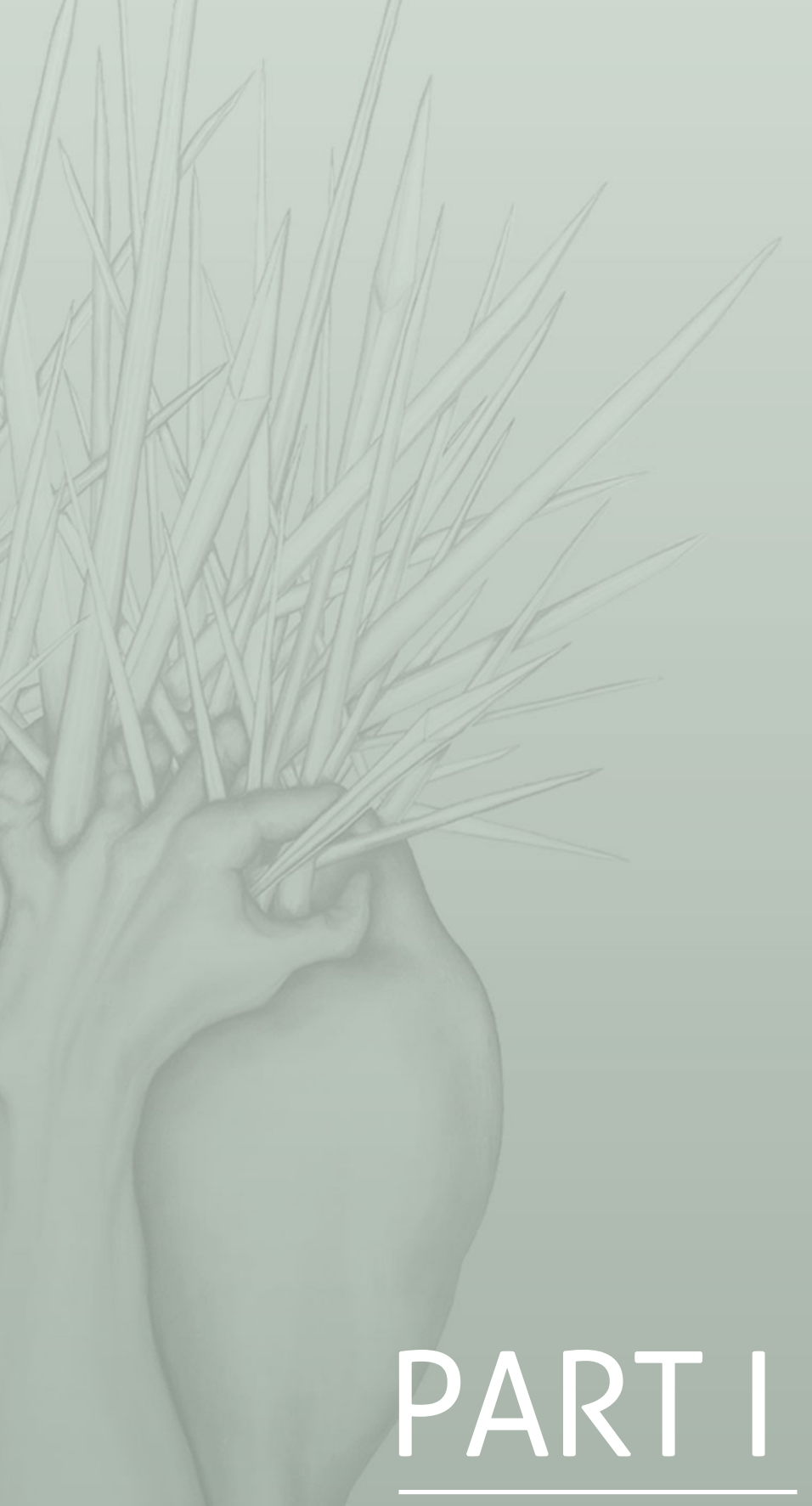

Uric acid and blood pressure: the role of uric acid production 



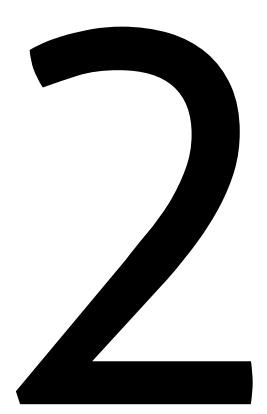

Xanthine oxidase gene variants and their association with blood pressure and incident hypertension: a population study

Lieke Scheepers, Fang-Fei Wei, Katarzyna Stolarz-Skrzypek, Sofia Malyutina, Valérie Tikhonoff, Lutgarde Thijs, Erika Salvi,

Cristina Barlassina, Jan Filipovský, Edoardo Casiglia, Yuri Nikitin, Kalina Kawecka-Jaszcz, Paolo Manunta, Daniele Cusi, Annelies Boonen, Jan Staessen, Ilja Arts 


\section{ABSTRACT}

\section{Objective}

The enzyme xanthine oxidoreductase (XOR) generates uric acid in the terminal steps of the purine metabolism; meanwhile reactive oxygen species are formed. We hypothesized that uric acid production, as assessed indirectly from XOR variants, is associated with hypertension.

\section{Methods}

Among 2769 participants (48.3\% men; mean age 40.7 years) randomly recruited from European populations, we genotyped 25 tagging XOR SNPs and measured blood pressure (BP) at baseline and follow-up (median 8.8 years). The relation between variants of the $X O R$ gene with changes in pulse pressure and mean arterial pressure over time; and incidence of hypertension, were analyzed.

\section{Results}

Compared with nonminor allele carriers, pulse pressure increased approximately 2 $\mathrm{mmHg}$ more in minor allele carriers of rs11904439 ( $P=0.01)$, whereas mean arterial pressure and DBP increased approximately $1 \mathrm{mmHg}$ less in minor allele carriers of rs2043013 $(P=0.01)$. In 2050, participants normotensive at baseline, hazard ratios contrasting risk of hypertension in minor allele carriers vs. nonminor allele carriers were 1.31 (95\% confidence interval 1.03-1.68; $P=0.02$ ) and 1.69 (95\% confidence interval 1.11-2.57; $P=0.01$ ) for rs11904439 and rs148756340, respectively. With the false discovery rate set at 0.25 , the aforementioned associations retained significance. The changes in SBP from baseline to follow-up and the serum levels of uric acid at baseline $(n=1949)$ were not associated with $X O R$.

\section{Conclusion}

Pending confirmation, our findings suggest that variation in uric acid production, as captured by genetic variation in $X O R$, might be a predictor of changes in $\mathrm{BP}$ and in the risk of hypertension. 
XOR gene variants and blood pressure $\mid 31$

\section{INTRODUCTION}

Cardiovascular disease is the leading cause of death worldwide and is an important contributor to years lived with disability. ${ }^{1,2}$ Hypertension is the most potent risk factor for cardiovascular disease. Globally, approximately 25-35\% of the adult population and more than $60 \%$ of the individuals over the age of 65 years have high blood pressure $(\mathrm{BP})^{3}$

$\mathrm{BP}$ and hypertension are associated with serum uric acid, the end product of purine catabolism, but whether this association is causal remains controversial. ${ }^{4}$ Some experts propose uric acid as a mere risk marker, because its serum concentration is often elevated in conditions other than hypertension, such as obesity or the metabolic syndrome. On the other hand, several studies suggested plausible mechanism by which uric acid may be causally linked to elevated BP and the development of hypertension. ${ }^{5}$

So far, epidemiological studies focused on the concentrations of uric acid in the serum. However, serum uric acid concentration is mainly determined by uric acid 'excretion' and not by its 'production'. ${ }^{6}$ During the last steps of uric acid production, xanthine oxidoreductase (XOR) catalyzes hypoxanthine to xanthine and xanthine to uric acid, thereby producing reactive oxygen species that inactivates the vasodilator nitric oxide (Figure 1). ${ }^{5}$ In this study, we hypothesized that not hyperuricemia as such, but increased production of uric acid by XOR elevates BP and leads to hypertension, as a consequence of oxidative stress, reduced availability of nitric oxide, and endothelial dysfunction. As we cannot measure uric acid production directly in a large cohort of healthy humans, we used variation in the XOR gene as a proxy. Several studies showed association of XOR expression with variation in the gene, and therefore, support this research approach. ${ }^{7.8}$ This study aimed to explore the role of XOR as a hypertension susceptibility gene by using 25 tagging single nucleotide polymorphisms (SNPs) in the XOR gene and by relating them to changes in $\mathrm{BP}$ over time and to the incidence of hypertension in $a$ European population study.

\section{PATIENTS AND METHODS}

\section{Study population}

Recruitment for the Flemish Study on Environment, Genes, and Health Outcomes (FLEMENGHO) started in $1985 .^{9}$

From August 1985 to November 1990, a random sample of the households living in a geographically defined area of northern Belgium was investigated with the goal to recruit an equal number of participants in each of six subgroups by sex and age (20-39, 40-59, and $\geq 60$ years). From June 1996 until January 2004, recruitment of families continued using the former participants (1985-1990) as index persons and also, including teenagers. ${ }^{9}$ Each participant underwent up to seven follow-up examinations. In 
$32 \mid$ Chapter 2

all study phases, we used the same standardized methods to measure clinical and biochemical variables, administer questionnaires, and to determine incidence of fatal and nonfatal outcomes. ${ }^{10}$ The European Project on Genes in Hypertension (EPOGH) recruited participants from 1999 to 2001 in four centers: Pilsen (Czech Republic), Mirano (Italy), Kraków (Poland), and Novosibirsk (Russian Federation). ${ }^{11}$ The EPOGH investigators received training at the Studies Coordinating Centre in Leuven, Belgium, and applied the same protocol, questionnaires, and follow-up procedures, as used in FLEMENGHO. ${ }^{11}$ In EPOGH, one follow-up examination took place from 2006 to 2008 and in FLEMENGHO, follow-up lasted until 2013. Both studies were conducted according to the principles outlined in the Helsinki declaration for investigation of human participants. ${ }^{12}$ Each local Institutional Review Board approved the study protocol. Participants provided written informed consent.

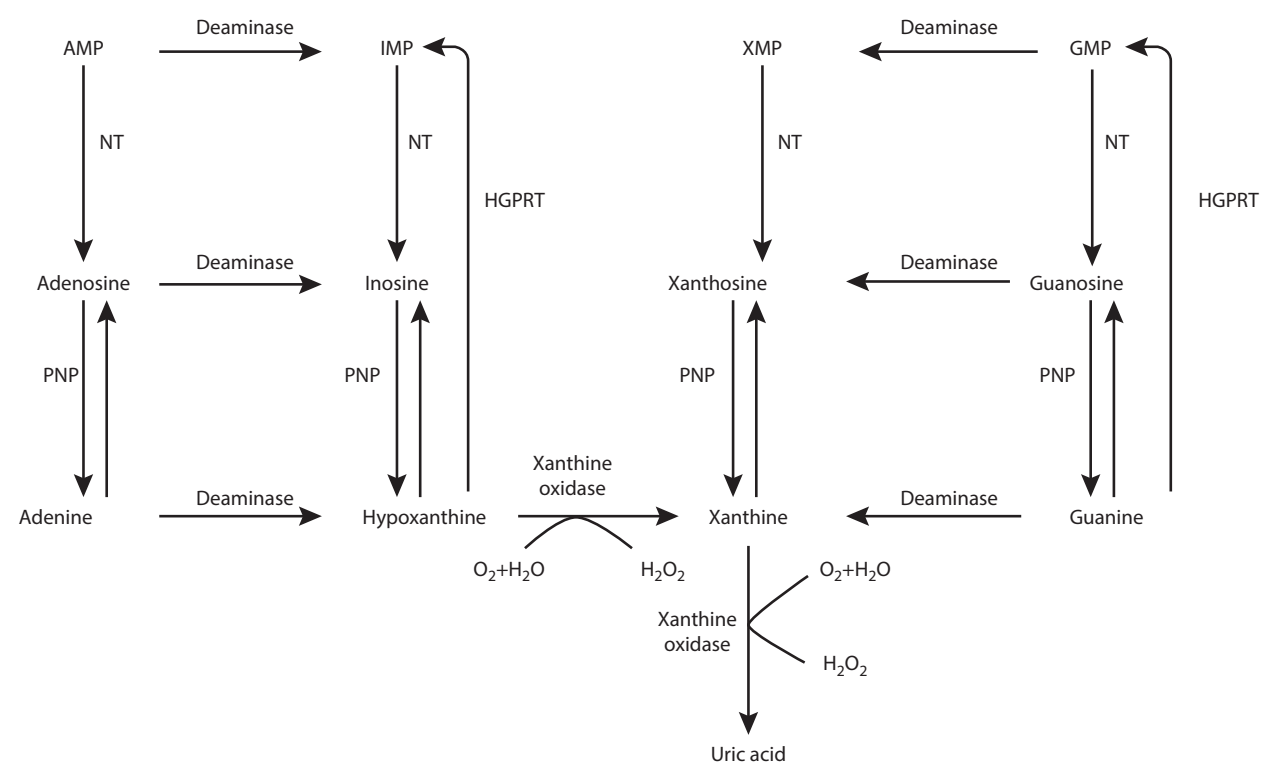

Figure 1. Purine metabolism. During the final stage of purine metabolism, xanthine oxidoreductase catalyzes hypoxanthine to xanthine, and xanthine to uric acid whereas reactive oxygen species is formed. These reactive oxygen species inactivate the vasodilator nitric oxide. HGPRT, hypoxanthine-guanine phosphoribosyltransferase; IMP, inosine monophosphate; NT, nucleotidase; PNP, purine nucleoside phosphorylase; XMP, Xanthosine monophosphate

\section{Definition of cohorts}

As shown in the flow chart (Figure 2), 3343 participants took place in FLEMENGHO and 1192 in EPOGH. Participants were excluded from analysis, if their BP measurements were missing at baseline $(n=61)$ or because XOR had not been genotyped $(n=1307)$ 
because of exhaustion of the biobank or DNA degradation. Of the remaining 3167 participants, 356 were not followed up and 42 had died. The 'blood pressure cohort' included 2769 participants to study the association between change in BP and genotype. The 'hypertension cohort' consisted of 2050 participants, who at baseline were normotensive and not on antihypertensive drug treatment.

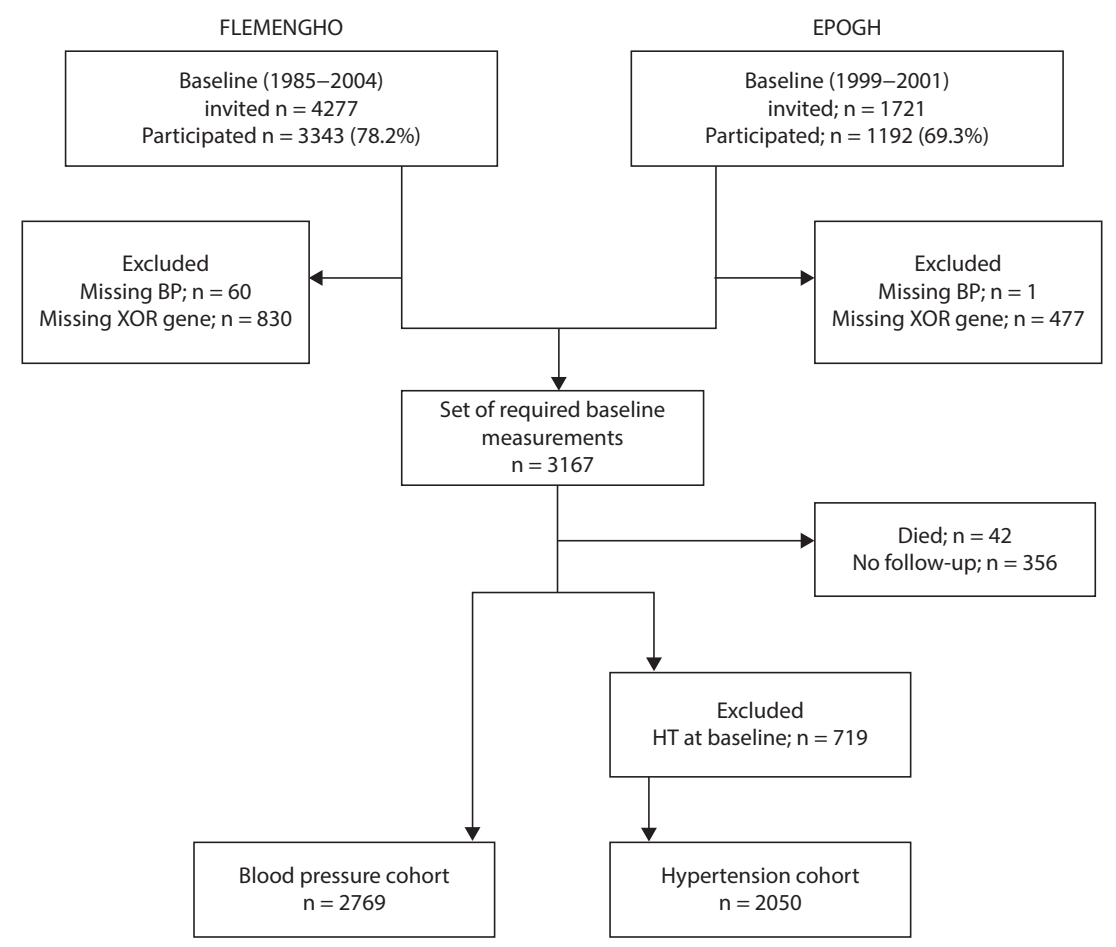

Figure 2. Flowchart of participants. The blood pressure cohort and hypertension cohort refer to participants used to study the association with change in BP and the incidence of HT over follow-up. BP, blood pressure; EPOGH, European Project on Genes in Hypertension; FLEMENGHO, Flemish Study on Environment, Genes, and Health Outcomes; HT, hypertension; XOR, xanthine oxidoreductase

\section{Measurements}

Experienced observers measured each participant's BP at baseline and follow-up by auscultation of the Korotkoff sounds. After the participant had rested for 5 min in the sitting position, they obtained five consecutive BP readings (phase $\mathrm{V} D B P$ ) to the nearest $2 \mathrm{mmHg}$, using mercury sphygmomanometers. Standard cuffs had a $12 \times 24 \mathrm{~cm}$ inflatable portion, but if upper arm girth exceeded $31 \mathrm{~cm}$, larger cuffs with $15 \times 35$ bladders were used. Previous publications describe the quality control of the BP measurements. ${ }^{11,13}$ 
34 Chapter 2

For analysis, the five $\mathrm{BP}$ readings were averaged. Hypertension was a $\mathrm{BP}$ equal to or exceeding $140 \mathrm{mmHg}$ SBP or $90 \mathrm{mmHg}$ DBP or use of antihypertensive drugs. We analyzed the pulsatile and steady component of BP as captured by pulse pressure (PP) and mean arterial pressure (MAP), respectively. $P P$ is the difference between SBP and DBP. MAP is DBP and one-third of PP. Combining PP and MAP is superior in addressing the relative contributions of both arterial stiffness and peripheral arterial resistance to cardiovascular risk. ${ }^{14}$ BP changes over time were calculated as the last follow-up minus the baseline measurement. Trained nurses measured anthropometric characteristics and administered the same questionnaire at baseline and follow-up to collect each participant's medical history, smoking status, drinking habits, and use of medications. BMI was weight in $\mathrm{kg} / \mathrm{m}^{2}$. Participants fasted for at least $6 \mathrm{~h}$ prior to venous blood sampling.

\section{Genotyping, imputation, and single-nucleotide polymorphisms selection}

The FLEMENGHO and EPOGH participants were genotyped at the University of Milan as part of the HYPERGENES project ${ }^{15}$ (European Network for Genetic-Epidemiological Studies; www.hypergenes.eu), using the $1 \mathrm{M}$ Duo chip, the Metabochip and/or a $15 \mathrm{~K}$ Custom chip (Illumina Inc., San Diego, California, USA). ${ }^{15}$ Imputation was performed using Minimac $^{16}$ and 1000 Genomes haplotypes as reference (release March 2012). With the 1M Duo chip, 87 XOR SNPs for 507 individuals were genotyped, six XOR SNPs for 994 individuals were genotyped with the Metabochip, and 20 XOR SNPs for 2742 individuals were genotyped with the $15 \mathrm{~K}$ custom chip. For each sample, 1299 SNPs were imputed, but only SNPs with an imputation quality of $r^{2}$ at least $80 \%$ were used in the association analysis. This resulted in 300 imputed SNPs for the $1 \mathrm{M}$ Duo array, none for the Metabochip, and 76 SNPs for the 15K.

XOR (80 423 base pairs) maps to chromosome 2 (2p23.1,2:31,557,187-31,637,611) (Figure Supplement 1 (S1)). To select the XOR SNPs to be included in the analyses, we first reviewed all SNPs in this gene, including the flanking regions $( \pm 20 \mathrm{~kb}$ from the gene). We excluded SNPs with a minor allele frequency of less than $1 \%$ or a genotyping call rate less than 0.99. We selected 28 tagging SNPs (rs1054889, rs207440, rs9308919, rs1896846, rs12621192, rs732436, rs2281548, rs17011368, rs2043013, rs10181969, rs11900892, rs45622435, rs11894627, rs45449798, rs45520334, rs3769618, rs4407290, rs2236168, rs148756340, rs561525, rs11904439, rs206857, rs10175754, rs206800, rs206803, rs3769616, rs206804, and rs206811) that were in high linkage disequilibrium $\left(r^{2} \geq 0.80\right)$ with 77 neighboring SNPs, but were not in high linkage disequilibrium $\left(r^{2} \geq 0.80\right)$ with each other (Supplemental Table S1). The 28 selected SNPs covered the entire gene with extension into the $3^{\prime}$ and 5 '-flanking regions. For analyses, the minor alleles were determined by using the European haplotype map population as reference (www.ncbi.nlm.nih.gov/snp). 
XOR gene variants and blood pressure $\mid 35$

\section{Statistical analysis}

For statistical analysis, we used SAS software version 9.3 (SAS Institute Inc., Cary, North Carolina, USA). We tested Hardy-Weinberg equilibrium in 1222 unrelated individuals, by using the exact statistics available in the PROC ALLELE procedure of the SAS package. We applied mixed models to investigate the association between BP changes over time and genotype, while accounting for family clusters and study center (FLEMENGHO or EPOGH) as random effects and adjusting for sex, age, BMI, BP at baseline, smoking and drinking alcohol, use of female sex hormones (all at baseline), duration of follow-up, and three indicator variables coding for starting or stopping antihypertensive drug treatment from baseline to follow-up or remaining on treatment. In 1949, participants whose serum uric acid concentration at baseline was known, we also examined its association with the genetic variants.

Next, we modelled the risk of hypertension in relation to the genetic variants by Cox proportional hazard regression analysis, as implemented in the PROC SURVIVAL procedure of the SUDAAN software (Research Triangle Institute, Research Triangle Park, North Carolina, USA) version 10.01. We censored study participants from further analysis after occurrence of the first diagnosis of hypertension. We checked the proportional hazards assumptions by the Kolmogorov-Smirnov supremum test. Hazard ratios were calculated for participants of the 'hypertension cohort,' while accounting for family clusters and study center as random effects. We additionally adjusted for sex, age, BMI, SBP and DBP, smoking and drinking alcohol, and use of female sex hormones (all at baseline).

In phenotype-genotype analyses, we contrasted minor allele carriers with nonminor allele carriers and we adjusted significance levels for multiple testing by BenjaminiHochberg's approach with the false discovery rate set at $0.25{ }^{17}$

\section{RESULTS}

The baseline characteristics of the study population by study center are shown in Table 1. The 'blood pressure cohort' included 2769 participants, of whom 1337 (48.3\%) were men. At baseline, participants were on average ( \pm SD) $40.7 \pm 15.2$ years old, and 719 had hypertension (26.0\%), of whom 322 (44.8\%) were on antihypertensive drug treatment. The 'hypertension cohort' included 2050 participants, of whom 969 (47.3\%) were men. Their mean age at baseline was $37.3 \pm 14.3$ years. All participants were white Europeans. 
$36 \mid$ Chapter 2

Table 1. Baseline characteristics of participants by cohort

\begin{tabular}{lcc}
\hline & Blood pressure cohort & Hypertension cohort \\
\hline Number of participants $(n)$ & 2769 & 2050 \\
Median follow-up years (IOR) & $8.8(6.7-12.8)$ & $9.1(6.7-14.0)$ \\
Number (\%) with characteristics & & \\
FLEMENGHO cohort & $2069(75.7)$ & $1631(79.6)$ \\
Men & $1337(48.3)$ & $969(47.3)$ \\
Hypertension & $719(26.0)$ & $0(0.0)$ \\
Antihypertensive drug use & $322(11.6)$ & $0(0.0)$ \\
Use of female sex hormones & $261(9.4)$ & $230(11.2)$ \\
Current smoking & $780(28.2)$ & $610(29.8)$ \\
Alcohol consumption & $930(33.6)$ & $659(32.2)$ \\
Uric acid lowering therapy & $17(0.6)$ & $5(0.2)$ \\
Mean (SD) of characteristic & & \\
Age (years) & $40.7(15.2)$ & $37.3(14.3)$ \\
BMI (kg/m ${ }^{2)}$ & $25.2(4.6)$ & $24.3(4.1)$ \\
SBP (mmHg) & $125.0(17.0)$ & $118.1(10.5)$ \\
DBP (mmHg) & $77.0(11.1)$ & $73.1(8.3)$ \\
Serum uric acid (mmol/L) ${ }^{a}$ & $293(84)$ & $286(81)$ \\
\hline
\end{tabular}

Blood pressure cohort and hypertension cohort refer to the participants used to study the changes in blood pressure and the incidence of hypertension over follow-up. Blood pressure was the average of five consecutive readings at a single visit. Hypertension is defined as untreated blood pressure of at least $140 \mathrm{mmHg} \mathrm{SBP}$, or $90 \mathrm{mmHg}$ DBP, or use of antihypertensive drugs. IQR, interquartile range; FLEMENGHO, Flemish Study on

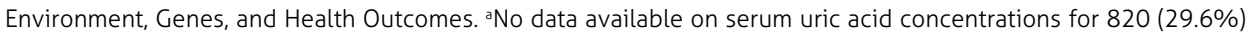
participants of the blood pressure cohort and 634 (30.9\%) participants of the hypertension cohort.

\section{Genotypes}

Of the 28 selected SNPs, 25 complied with Hardy-Weinberg equilibrium ( $P$-value $>0.10$ ) and were included in the analyses (Supplemental Table S2). The frequencies of the minor alleles ranged from $1.7 \%$ for rs 148756340 and rs 455203344 to $48.6 \%$ for rs 1054889 .

\section{Longitudinal changes in mean arterial pressure and pulse pressure}

Follow-up of BP was available at one, two, or at least three occasions for 2769, 1450, and 1035 participants, respectively. PP changes (follow-up minus baseline) over a median of 8.8 years (interquartile range, 6.7-12.8) in minor allele carriers ranged from $1.7 \mathrm{mmHg}$ for rs4407290 to $5.0 \mathrm{mmHg}$ for rs11904439 (Supplemental Table S3) In nonminor allele carriers, this range encompassed $1.9 \mathrm{mmHg}$ for rs11904439 to $2.3 \mathrm{mmHg}$ for rs732436. In multivariable-adjusted analyses, a significant difference in the PP change over time (nonminor allele carriers minus minor allele carriers) was associated with rs11904439 [2.09 mmHg; 95\% confidence interval (Cl), 0.43-3.75 mmHg] (Supplemental Table S3).

Furthermore, MAP changes in minor allele carriers ranged from $2.8 \mathrm{mmHg}$ for rs148756340 to $4.9 \mathrm{mmHg}$ for rs206803 (Supplemental Table S3). In nonminor allele carriers, the corresponding range encompassed $3.9 \mathrm{mmHg}$ for rs2236168 to $4.8 \mathrm{mmHg}$ for rs2043013. In multivariable-adjusted analyses, a significant difference in MAP changes (nonminor allele carriers minus minor allele carriers) was observed for 
rs12621192 (-0.78 mmHg; $\mathrm{Cl}, 1.52$ to $-0.04 \mathrm{mmHg}$; $P=0.04$ ) and rs2043013 ('0.99 mmHg; $\mathrm{Cl},-1.77$ to $-0.20 \mathrm{mmHg} ; P=0.01$ ). The MAP results for rs2043013 depended on a lesser increase in DBP in minor allele carriers compared with nonminor allele carriers (-1.06 mmHg; $\mathrm{Cl},-1.82$ to -0.30 mmHg; $P$-value <0.01) (Supplemental Table S3).

With Benjamini-Hochberg's correction for multiple testing applied, the aforementioned associations of PP with rs11904439 and of MAP and DBP with rs2043013 retained significance. Even without Benjamini-Hochberg's correction, none of the associations of changes in SBP or the serum levels of uric acid at baseline $(n=1949)$ with XOR variants was significant $(P$-value $\geq 0.05)$.

\section{Incidence of hypertension}

Follow-up for incident hypertension was available at one, two, or at least three occasions in 2050, 1119, and 805 participants, respectively. The median number of follow-up years was 9.1 years (interquartile range, 6.7-14.0). In the entire 'hypertension cohort', 753 (36.4\%) participants who were normotensive at baseline developed hypertension. Of these, 230 (30.8\%) were on antihypertensive drug treatment. In the remaining 517 patients $(69.2 \%)$, the diagnosis of hypertension relied on thresholds being exceeded for $\operatorname{SBP}(n=188 ; 25.0 \%), \operatorname{DBP}(n=186 ; 24.7 \%)$, or both $(n=149 ; 19.8 \%)$.

The hazard ratios contrasting the risk of hypertension in minor allele carriers vs. nonminor allele carriers ranged from $0.90(\mathrm{Cl}, 0.77-1.06 ; P=0.21)$ for rs9308919 to 1.31 $(\mathrm{Cl}, 1.03-1.68 ; P=0.02)$ and to $1.69(\mathrm{Cl}, 1.11-2.57 ; P=0.01)$ for $r s 11904439$ and rs148756340, respectively (Figure 3). With Benjamini-Hochberg's correction for multiple testing applied, the hazard ratios remain significant for rs11904439 and rs148756340 (Supplemental Table S4).

(a)

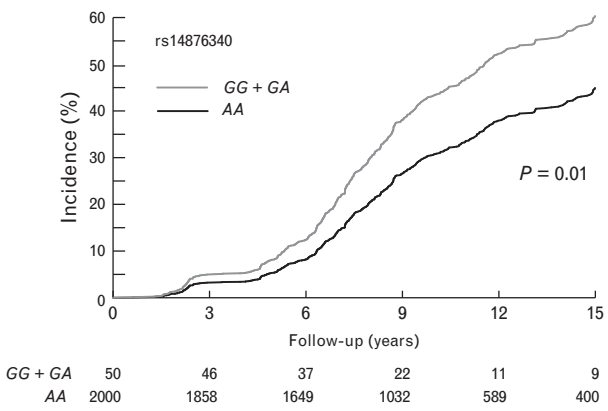

(b)

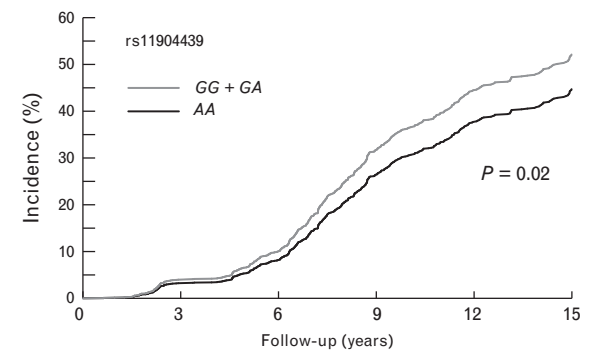

\begin{tabular}{rrrrrrr}
$G G+G A$ & 159 & 146 & 130 & 80 & 43 & 31 \\
\hline & 1891 & 1758 & 1156 & 974 & 557 & 378
\end{tabular}

Figure 3. Cumulative incidence plots. Sex and age-standardized cumulative incidence of hypertension in relation to rs148756340 (a) and rs11904439 (b) XOR genotypes. XOR, xanthine oxidoreductase. 


\section{DISCUSSION}

In the present study, using genetic variation in XOR as a proxy, we tested the hypothesis that variation in the uric acid production might be associated with secular trends in the steady and pulsatile BP components and the risk of hypertension. Accepting a false discovery rate of 0.25 , the increase in PP and MAP and the risk of hypertension over 8-9 years of follow-up (median) were associated with three of 25 SNPs in XOR. Compared with nonminor allele carriers, PP increased by approximately $2 \mathrm{mmHg}$ more in minor allele carriers of rs11904439, whereas MAP and DBP increased approximately $1 \mathrm{mmHg}$ less in minor allele carriers of rs2043013. The risk of hypertension was $30-70 \%$ higher in minor allele carriers of rs11904439 and rs148756340, respectively. The XOR enzyme is responsible for the two final steps of purine degradation, at which hypoxanthine is converted to xanthine and xanthine to uric acid (Figure 1). The present study therefore lends some support for the hypothesis underlying its rational.

It is generally accepted that an association between uric acid and BP exists, and Johnson and coworkers ${ }^{18}$ have been key investigators in studying this association. They showed in animal models ${ }^{5}$ and observational studies ${ }^{19}$ that elevated uric acid concentrations are associated with elevated BP. The meta-analysis performed by Grayson et al. ${ }^{20}$ in 2011 , which included data from 57,607 participants of 18 prospective cohort studies, has shown that hyperuricemia is associated with an increased risk for incident hypertension, independent of traditional hypertension risk factors (adjusted relative risk ratio $1.41 ; \mathrm{Cl}$, 1.23-1.58). Furthermore, for a $1 \mathrm{mg} / \mathrm{dL}$ increase in uric acid concentrations, the pooled adjusted relative risk for incident hypertension was $1.13(\mathrm{Cl}, 1.06-1.20){ }^{20}$

Despite this body of evidence, Mendelian randomization studies aiming to establish causality have not been able to convincingly demonstrate that the association between uric acid and BP is causal. Only three ${ }^{21-23}$ out of eight studies ${ }^{21-28}$ showed a significant association between polymorphisms of the SLC2A9 gene, a robust proxy for uric acid concentrations, and BP. Furthermore, a meta-analysis of randomized controlled trials to assess the efficacy of the XOR inhibitor allopurinol on BP showed that allopurinol treatment decreases SBP by $3.3 \mathrm{mmHg}(\mathrm{Cl}, 1.4-5.3 \mathrm{mmHg})$ and DBP by $1.3 \mathrm{mmHg}(\mathrm{Cl}$, 0.1-2.5 mmHg). ${ }^{29}$ Allopurinol furthermore improved endothelial-dependent vasodilation, whereas the uricosuric drug probenecid did not. ${ }^{30}$ These findings further suggest that next to uric acid concentrations, the role of uric acid production by XOR should be considered as a determinant of BP.

XOR is expressed in many tissues but its activity is strongest in the liver and the gut. ${ }^{31,32}$ Individual differences in the activity of human liver XOR exist. XOR activity depends on sex, age, and ethnicity. Men show stronger hepatic XOR activity than women do and the enzymatic activity increases with advancing age. ${ }^{33-35}$

So far, genetic variation in the XOR gene has not been studied extensively. ${ }^{7,86-39}$ Most of the available studies focused on genetic variants that cause xanthine oxidase deficiency, also known as the rare disease xanthinuria type.$^{36-39}$ Two in-vitro studies from Kudo et al. ${ }^{7,8}$ addressed the impact of variation in the $X O R$ gene on xanthine 
oxidase activity. Molecular studies of the DNA of 96 Japanese, revealed three nonsynonymous SNPs. Functional characterization of 21 XOR variants revealed deficient enzyme activity in two, and reduced and enhanced activity in six and two, respectively. ${ }^{7}$ Among 13 SNPs in the XOR 5'-flanking region, one was associated with decreased expression in transfected cells. ${ }^{8}$ Both studies examined polymorphisms of the XOR gene in a Japanese population and as its activity might be influenced by ethnicity, caution is required when applying these results to a European study population. ${ }^{7,8}$ The SNPs investigated in the Japanese studies were not determined and/or in linkage disequilibrium with the SNPs from the current study.

So far, only two candidate gene studies investigated the association between BP and XOR variants. ${ }^{40,41}$ The cross-sectional study from Yang et al. ${ }^{40}$ investigated the genetic variation in the promotor and all exon regions of the XOR gene in 48 randomly chosen Japanese patients with hypertension. They identified three missense mutations (G172R, $\mathrm{A} 932 \mathrm{~T}$, and $\mathrm{N} 1109 \mathrm{~T}$ ) in a heterozygous state in addition to 34 variations, including 15 common SNPs in 48 hypertensive patients. Additionally, 953 hypertensive patients and 1818 patients from the general Japanese population were genotyped for three missense mutations and eight common SNPs. Four hypertensive patients with rare missense mutations (G172R or N1109T) in homozygous form had resistant hypertension, despite antihypertensive drug therapy. Multivariable adjusted logistic regression showed a significant association of three SNPs with hypertension in men: 47686C $>T$ [ [rs229475; odds ratio (OR), 1.52; $P=0.047$ ] and 69901A $>C$ (intron 31; $O R, 3.14 ; P=0.039$ ) in the recessive model and 67873A $>C$ (N1109T; rs45547640; OR, 1.84; $P=0.018$ ) in the dominants model. SNP rs2295475 was also present in our dataset and in high linkage disequilibrium with SNP rs12621192. The latter was retained for analysis but was not associated with any of the outcomes considered. As our study included white Europeans and was longitudinal instead of cross-sectional, caution is required by comparing our result with the previous studies. ${ }^{40}$

The genome-wide association studies (GWAS) published so far on SBP, DBP, and hypertension, ${ }^{42-45}$ and on PP and MAP ${ }^{46-48}$ identified numerous novel loci, but none of these were located near the XOR gene. Cross-sectional GWAS studies offer the opportunity for searching for associations between BP and densely distributed SNPs across the whole genome in large numbers of study participants. Such studies require significance levels of $10^{-6}$ to $10^{-9}$. In contrast, our study was prospective and population based, involving only 25 common tagging SNPs in XOR gene. We did, therefore, not rely on such extreme $P$ values, but applied the Benjamin-Hochberg's approach for multiple testing. Admittedly, our sample size was smaller than in GWAS studies. As the functionality of the SNPs is unknown, caution is required by interpreting the results and the present findings can only be considered as hypothesis generating. Future population-based research projects might address this tissue.

The prospective study design, genotyping of 25 common tagging SNPs in XOR gene, the extensive and reliable phenotyping of BP, and adjustment for potential confounders are major strengths of our study. Nevertheless, our current study must be interpreted within 
$40 \mid$ Chapter 2

the context of its limitations. First, the functionality of the tagging SNPs analyzed in our current study remains unknown. Second, we accepted a false discovery rate of 0.25 , so that the chance of rejecting a true-positive SNP is low, however, $25 \%$ of the significant results might be wrong. Therefore, further investigation is required to confirm our findings. Third, our sample size was relatively small $(n=2796)$ and might have been insufficient to identify SNPs associated with BP in a reliable manner. However, if a larger sample would be required, it follows that the effects on BP must be minute. Finally, our current findings are probably representative for white Europeans, but should not be extrapolated to other ethnic groups or people with a different lifestyle with possible epigenetic impact on gene expression or functionality.

In conclusion, we demonstrated that BP rise over time and/or the risk of hypertension are associated with three SNP in the XOR gene: rs2043013, rs11904439, and rs148756340. Given the burden of chronic hypertension and the fact that only $37 \%$ of the patients obtain a well managed $\mathrm{BP}^{49}$ a better understanding of these associations should be subject to further experimental, clinical, and epidemiologic research. Further experimental research is required to examine whether the identified SNPs from the present study alter enzyme activity. Previous studies have shown that genetic variation in other genes, even if this is in the intron, can influence gene regulation. For example, by altering transcription activity ${ }^{50}$ or the role of RNAs derived from introns. ${ }^{51,52}$ Moreover, our current findings need replication in other longitudinal studies in patients and populations. In view of the role of circulating uric acid as a marker of the risk of death and cardiovascular complications, ${ }^{53}$ further studies should also address the association between incidence cardiovascular disease and variation in the XOR gene. Pending confirmation, the rs2043013, rs11904439, and rs148756340 polymorphisms might then be used to identify overproducers of uric acid; and combined with other genetic markers might contribute to the stratification of cardiovascular risk. 
\begin{tabular}{l|l} 
XOR gene variants and blood pressure & \\
41
\end{tabular}

\section{REFERENCES}

1. Murray C], Lopez AD. Measuring the global burden of disease. N Engl J Med. 2013; 369:448457.

2. Lozano R, Naghavi M, Foreman K, et al. Global and regional mortality from 235 causes of death for 20 age groups in 1990 and 2010: a systematic analysis for the Global Burden of Disease Study 2010. Lancet. 2012; 380:20952128.

3. Wolf-Maier K, Cooper RS, Banegas JR, et al. Hypertension prevalence and blood pressure levels in 6 European countries, Canada, and the United States. JAMA. 2003; 289:2363-2369.

4. Feig DI, Madero M, Jalal DI, et al. Uric acid and the origins of hypertension. J Pediatr. 2013; 162:896-902.

5. Mazzali M, Hughes J, Kim YG, et al. Elevated uric acid increases blood pressure in the rat by a novel crystalindependent mechanism. Hypertension. 2001; 38:1101-1106.

6. Richette P, Bardin T. Gout. Lancet. 2010; 375:318-328.

7. Kudo M, Moteki T, Sasaki T, et al. Functional characterization of human xanthine oxidase allelic variants. Pharmacogenet Genomics. 2008; 18:243-251.

8. Kudo M, Sasaki T, Ishikawa M, et al. Functional characterization of genetic polymorphisms identified in the promoter region of the xanthine oxidase gene. Drug Metab Pharmacokinet. 2010; 25:599-604.

9. Staessen JA, Wang JG, Brand E, et al. Effects of three candidate genes on prevalence and incidence of hypertension in a Caucasian population. J Hypertens. 2001; 19:1349-1358.

10. Li Y, Zagato L, Kuznetsova T, Tripodi G, et al. Angiotensin-converting enzyme I/D and alphaadducin Gly460Trp polymorphisms: from angiotensin-converting enzyme activity to cardiovascular outcome. Hypertension. 2007; 49:1291-1297.

11. Kuznetsova T, Staessen JA, Kawecka-Jaszcz K, et al. Quality control of the blood pressure phenotype in the European Project on Genes in Hypertension. Blood Press Monit. 2002; 7:215224.

12. World Medical Association. World Medical Association Declaration of Helsinki. Ethical principles for medical research involving human subjects. Bull World Health Organ. 2001; 79:373-374.
13. Staessen J, Bulpitt C], Fagard R, et al. Familial aggregation of blood pressure, anthropometric characteristics and urinary excretion of sodium and potassium: a population study in two Belgian towns. J Chronic Dis. 1985; 38:397-407.

14. Franklin SS, Lopez VA, Wong ND, et al. Single versus combined blood pressure components and risk for cardiovascular disease: the Framingham Heart Study. Circulation. 2009; 119:243-250.

15. Salvi E, Kutalik Z, Glorioso N, et al. Genomewide association study using a high-density single nucleotide polymorphism array and case-control design identifies a novel essential hypertension susceptibility locus in the promoter region of endothelial NO synthase. Hypertension. 2012; 59:248-255.

16. Howie B, Fuchsberger C, Stephens M, et al. Fast and accurate genotype imputation in genomewide association studies through prephasing. Nat Genet. 2012; 44:955-959.

17. Benjamini $Y$, Hochberg $Y$. Controlling the false discovery rate: a practical and powerful approach to multiple testing. J R Stat Soc Series $B$ (Methodological). 1995; 57:289-300.

18. Johnson RJ. Why focus on uric acid? Curr Med Res Opin. 2015; 31 (Suppl 2):3-7.

19. Bjornstad P, Wadwa RP, Sirota JC, et al. Serum uric acid and hypertension in adults: a paradoxical relationship in type 1 diabetes. J Clin Hypertens. 2014; 16:283-288.

20. Grayson PC, Kim SY, LaValley $M$, et al. Hyperuricemia and incident hypertension: a systematic review and meta-analysis. Arthritis Care Res (Hoboken). 2011; 63:102-110.

21. Mallamaci F, Testa A, Leonardis D, et al. A polymorphism in the major gene regulating serum uric acid associates with clinic SBP and the white-coat effect in a family-based study. J Hypertens. 2014; 32:1621-1628.

22. Mallamaci $F$, Testa $A$, Leonardis $D$, et al. A genetic marker of uric acid level, carotid atherosclerosis, and arterial stiffness: a familybased study. Am J Kidney Dis. 2015; 65:294-302.

23. Parsa A, Brown E, Weir MR, et al. Genotypebased changes in serum uric acid affect blood pressure. Kidney Int. 2012; 81:502-507.

24. Caulfield MJ, Munroe PB, O’Neill D, et al. SLC2A9 is a high-capacity urate transporter in humans. PLoS Med. 2008; 5:1509-1523.

25. Han X, Gui L, Liu B, et al. Associations of the uric acid related genetic variants in SLC2A9 and 
$42 \mid$ Chapter 2

$A B C G 2$ loci with coronary heart disease risk. BMC Genet. 2015; 16:1-7.

26. Palmer TM, Nordestgaard BG, Benn $M$, et al. Association of plasma uric acid with ischaemic heart disease and blood pressure: mendelian randomisation analysis of two large cohorts. BMJ. 2013; 347:f4262.

27. Vitart V, Rudan I, Hayward C, et al. SLC2A9 is a newly identified urate transporter influencing serum urate concentration, urate excretion and gout. Nat Genet. 2008; 40:437-442.

28. Yang $\mathrm{O}$, Kottgen A, Dehghan A, et al. Multiple genetic loci influence serum urate levels and their relationship with gout and cardiovascular disease risk factors. Circ Cardiovasc Genet. 2010; 3:523-530.

29. Agarwal V, Hans N, Messerli FH. Effect of allopurinol on blood pressure: a systematic review and meta-analysis. J Clin Hypertens (Greenwich). 2013; 15:435-442.

30. George ], Carr E, Davies ], et al. High-dose allopurinol improves endothelial function by profoundly reducing vascular oxidative stress and not by lowering uric acid. Circulation. 2006; 114:2508-2516.

31. Xu P, Huecksteadt TP, Harrison R, et al. Molecular cloning, tissue expression of human xanthine dehydrogenase. Biochem Biophys Res Commun. 1994; 199:998-1004.

32. Saksela M, Lapatto R, Raivio KO. Xanthine oxidoreductase gene expression and enzyme activity in developing human tissues. Biol Neonate. 1998; 74:274-280.

33. Aklillu E, Carrillo JA, Makonnen E, et al. Xanthine oxidase activity is influenced by environmental factors in Ethiopians. Eur J Clin Pharmacol. 2003; 59:533-536.

34. Guerciolini R, Szumlanski C, Weinshilboum RM. Human liver xanthine oxidase: nature and extent of individual variation. Clin Pharmacol Ther. 1991; 50:663-672.

35. Aranda R, Domenech E, Rus AD, et al. Agerelated increase in xanthine oxidase activity in human plasma and rat tissues. Free Radic Res. 2007; 41:1195-1200.

36. Gok F, Ichida K, Topaloglu R. Mutational analysis of the xanthine dehydrogenase gene in a Turkish family with autosomal recessive classical xanthinuria. Nephrol Dial Transplant. 2003; 18:2278-2283.

37. Levartovsky D, Lagziel A, Sperling O, et al. XDH gene mutation is the underlying cause of classical xanthinuria: a second report. Kidney Int. 2000; 57:2215-2220.
38. Sakamoto N, Yamamoto T, Moriwaki Y, et al. Identification of a new point mutation in the human xanthine dehydrogenase gene responsible for a case of classical type I xanthinuria. Hum Genet. 2001; 108:279-283.

39. Stiburkova B, Krijt J, Vyletal $P$, et al. Novel mutations in xanthine dehydrogenase/oxidase cause severe hypouricemia: biochemical and molecular genetic analysis in two Czech families with xanthinuria type I. Clin Chim Acta. 2012; 413:93-99.

40. Yang J, Kamide K, Kokubo Y, et al. Associations of hypertension and its complications with variations in the xanthine dehydrogenase gene. Hypertens Res. 2008; 31:931-940.

41. Chaves FJ, Corella D, Blesa S, et al. Xanthine oxidoreductase polymorphisms: influence in blood pressure and oxidative stress levels. Pharmacogenet Genomics. 2007; 17:589-596.

42. Levy D, Ehret GB, Rice K, et al. Genome-wide association study of blood pressure and hypertension. Nat Genet. 2009; 41:677-687.

43. Newton-Cheh C, Johnson T, Gateva V, et al. Genome-wide association study identifies eight loci associated with blood pressure. Nat Genet. 2009; 41:666-676.

44. Kato N, Takeuchi F, Tabara Y, et al. Metaanalysis of genome-wide association studies identifies common variants associated with blood pressure variation in east Asians. Nat Genet. 2011; 43:531-538.

45. Ehret GB, Munroe PB, Rice KM, et al. Genetic variants in novel pathways influence blood pressure and cardiovascular disease risk. Nature. 2011; 478:103-109.

46. Kelly TN, Takeuchi F, Tabara Y, et al. Genomewide association study meta-analysis reveals transethnic replication of mean arterial and pulse pressure loci. Hypertension. 2013;62:853859.

47. Wain LV, Verwoert GC, O'Reilly PF, et al. Genome-wide association study identifies six new loci influencing pulse pressure and mean arterial pressure. Nat Genet. 2011;43:10051011.

48. Leu HB, Chung CM, Lin S], et al. A novel SNP associated with nighttime pulse pressure in young-onset hypertension patients could be a genetic prognostic factor for cardiovascular events in a general cohort in Taiwan. PLoS One. 2014; 9:e97919.

49. Falaschetti E, Mindell J, Knott C, et al. Hypertension management in England: a serial cross-sectional study from 1994 to 2011. Lancet. 2014; 383:1912-1919. 
\begin{tabular}{l|l} 
XOR gene variants and blood pressure & \\
43
\end{tabular}

50. Salvi E, Kuznetsova T, Thijs L, et al. Target sequencing, cell experiments, and a population study establish endothelial nitric oxide synthase (eNOS) gene as hypertension susceptibility gene. Hypertension. 2013; 62:844-852.

51. Osman I, Tay ML, Pek JW. Stable intronic sequence RNAs (sisRNAs): a new layer of gene regulation. Cell Mol Life Sci. 2016; 26:25502559.
52. Cech TR, Steitz JA. The noncoding RNA revolution-trashing old rules to forge new ones. Cell. 2014; 157:77-94.

53. Wang J-G, Staessen JA. Raised concentrations of serum creatinine and uric acid and the risks of mortality and cardiovascular disease. Cardiovasc Res Rep. 2002; 23:393-399. 
$44 \mid$ Chapter 2

\section{SUPPLEMENTAL MATERIAL}

Table S1. 25 Common Tagger SNPs in XOR Gene that are in High Linkage Disequilibrium $\left(r^{2} \geq 0.80\right)$ with 77 Other SNPS

\begin{tabular}{|c|c|c|c|c|}
\hline SNP ID & $\begin{array}{l}\text { Position } \\
\text { (Base-Pair) }\end{array}$ & Location & MAF & Tagged SNP \\
\hline rs1054889 & 31557308 & 3utr & 0.48 & $\begin{array}{l}\text { rs1042039, rs2268800, rs10490361, rs932559, rs736492, } \\
\text { rs207429, rs12614330, rs169596 }\end{array}$ \\
\hline rs207440 & 31562412 & coding & 0.05 & rs207445 \\
\hline rs9308919 & 31568726 & intronic & 0.20 & $\begin{array}{l}\text { rs4952083, rs7598968, rs7572658, rs7599175, } \\
\text { rs1366811, rs1366813, rs4952084, rs4952085, } \\
\text { rs1884725, rs13415401, rs7557095 }\end{array}$ \\
\hline rs12621192 & 31588664 & intronic & 0.33 & rs2295475, rs761926 \\
\hline rs732436 & 31590156 & intronic & 0.24 & rs2365842, rs1429376 \\
\hline rs17011368 & 31590917 & coding & 0.03 & $\begin{array}{l}\text { rs } 45565237, \text { rs } 17395161, \text { rs } 17323225, \text { rs } 45443498, \\
\text { rs } 115074795, \text { rs } 17395224, \text { rs } 45449798, \text { rs } 114281245, \\
\text { rs } 45550432, \text { rs } 45457594, \text { rs } 116648715\end{array}$ \\
\hline rs2043013 & 31592323 & intronic & 0.43 & rs1366817, rs2070293 \\
\hline rs10181969 & 31593125 & intronic & 0.03 & - \\
\hline rs11900892 & 31594524 & intronic & 0.03 & - \\
\hline rs13398137 & 31594789 & intronic & 0.06 & rs45596941, rs45445692, rs7597755 \\
\hline rs11894627 & 31597410 & intronic & 0.44 & $\begin{array}{l}\text { rs4952088, rs4952087, rs4952086, rs4952089, } \\
\text { rs6720163, rs1864280 }\end{array}$ \\
\hline rs45449798 & 31598810 & intronic & 0.02 & $\begin{array}{l}\text { rs17395224, rs114281245, rs115074795, rs45443498, } \\
\text { rs17323225, rs45550432, rs45457594, rs116648715, } \\
\text { rs17395161, rs17011368, rs45565237 }\end{array}$ \\
\hline rs45520334 & 31602147 & intronic & 0.02 & - \\
\hline rs3769618 & 31605447 & intronic & 0.35 & rs4952089, rs2281547 \\
\hline rs4407290 & 31606670 & coding & 0.03 & - \\
\hline rs2236168 & 31607128 & intronic & 0.46 & - \\
\hline rs148756340 & 31607275 & intronic & 0.01 & - \\
\hline rs561525 & 31608855 & intronic & 0.02 & rs473935, rs528551, rs206856 \\
\hline rs11904439 & 31614900 & intronic & 0.04 & - \\
\hline rs206857 & 31619101 & intronic & 0.16 & rs13418515 \\
\hline rs10175754 & 31621598 & intronic & 0.13 & rs13431382, rs10199855, rs11895133, rs13418515 \\
\hline rs206800 & 31627947 & intronic & 0.20 & - \\
\hline rs206803 & 31629030 & intronic & 0.14 & rs206798, rs494852, rs1366814, rs6759319, rs114978564 \\
\hline rs3769616 & 31630382 & intronic & 0.03 & rs111749408, rs76333723 \\
\hline rs206804 & 31630846 & intronic & 0.16 & rs206805, rs206798, rs494852 \\
\hline
\end{tabular}

SNP, single-nucleotide polymorphism; MAF: minor allele frequency. SNP ID is a GenBank ID number (NCBI). Position and location type were taken from the most recent human genome sequence assemblies (NCBI Build 37.3). MAFs were calculated in the present population. 
\begin{tabular}{l|l} 
XOR gene variants and blood pressure & \\
45
\end{tabular}

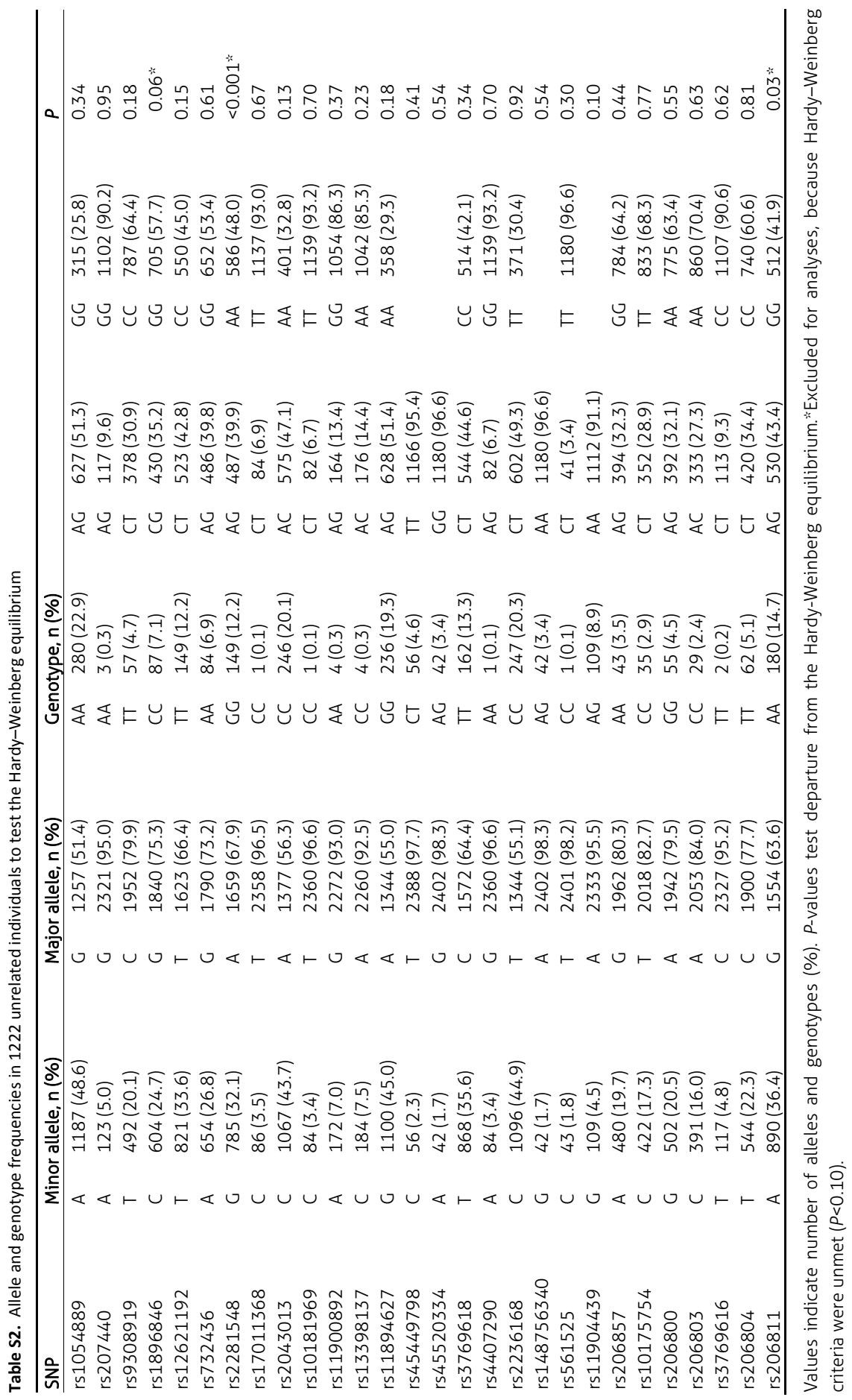


$46 \mid$ Chapter 2

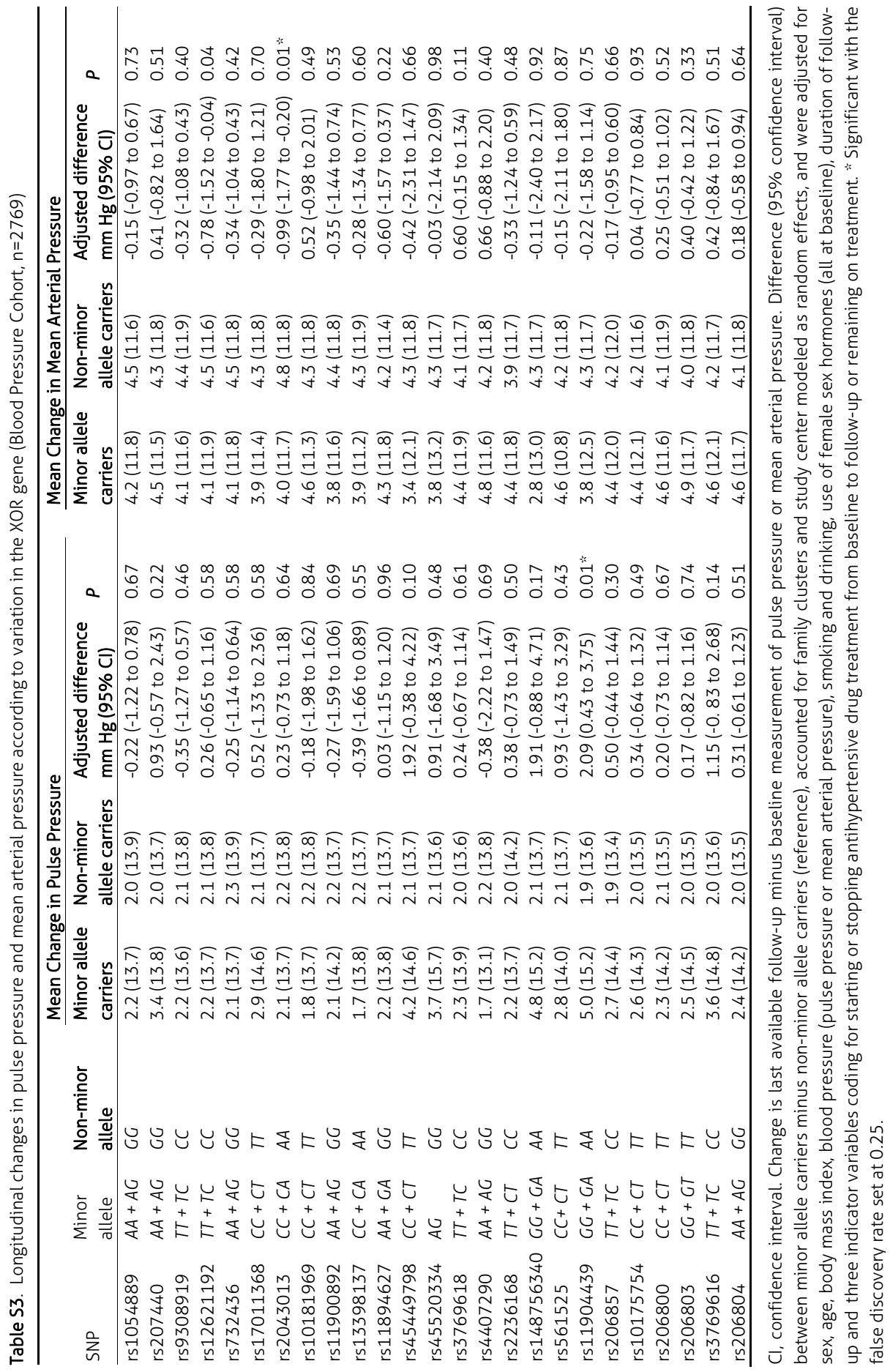


Table S4. Hazard Ratios Relating Risk of Hypertension to Variation in the XOR Gene (Hypertension Cohort, $n=2050$ )

\begin{tabular}{lcccc}
\hline SNP & Minor allele carriers & $\begin{array}{c}\text { Non-minor allele } \\
\text { Carriers }\end{array}$ & $\begin{array}{c}\text { Adjusted Hazard Ratio }(95 \% \\
\text { Confidence Interval) }\end{array}$ & $P$ \\
\hline rs1054889 & $A A+A G$ & $G G$ & $1.03(0.88$ to 1.21$)$ & 0.72 \\
rs207440 & $A A+A G$ & $G G$ & $0.93(0.70$ to 1.25$)$ & 0.64 \\
rs9308919 & $T T+T C$ & $C C$ & $0.90(0.77$ to 1.06$)$ & 0.21 \\
rs12621192 & $T T+T C$ & $C C$ & $0.97(0.83$ to 1.13$)$ & 0.70 \\
rs732436 & $A A+A G$ & $G G$ & $0.92(0.79$ to 1.06$)$ & 0.25 \\
rs17011368 & $C C+C T$ & $T T$ & $0.94(0.67$ to 1.32$)$ & 0.73 \\
rs2043013 & $C C+C A$ & $A A$ & $1.05(0.86$ to 1.27$)$ & 0.64 \\
rs10181969 & $C C+C T$ & $T T$ & $1.01(0.79$ to 1.30$)$ & 0.92 \\
rs11900892 & $A A+A G$ & $G G$ & $1.11(0.89$ to 1.39$)$ & 0.35 \\
rs13398137 & $C C+C A$ & $A A$ & $0.99(0.81$ to 1.22$)$ & 0.93 \\
rs11894627 & $A A+G A$ & $G G$ & $1.00(0.83$ to 1.20$)$ & 0.98 \\
rs45449798 & $C C+C T$ & $T T$ & $1.34(0.92$ to 1.94$)$ & 0.13 \\
rs45520334 & $A G$ & $G G$ & $1.28(0.87$ to 1.90$)$ & 0.22 \\
rs3769618 & $T T+T C$ & $C C$ & $1.01(0.87$ to 1.17$)$ & 0.93 \\
rs4407290 & $A A+A G$ & $G G$ & $0.92(0.69$ to 1.22$)$ & 0.55 \\
rs2236168 & $T T+C T$ & $C C$ & $1.03(0.87$ to 1.24$)$ & 0.79 \\
rs148756340 & $G G+G A$ & $A A$ & $1.69(1.11$ to 2.57$)$ & $0.01 *$ \\
rs561525 & $C C+C T$ & $T T$ & $1.20(0.79$ to 1.80$)$ & 0.39 \\
rs11904439 & $G G+G A$ & $A A$ & $1.31(1.03$ to 1.68$)$ & $0.02 *$ \\
rs206857 & $T T+T C$ & $C C$ & $1.14(0.97$ to 1.34$)$ & 0.12 \\
rs10175754 & $C C+C T$ & $T T$ & $1.11(0.94$ to 1.31$)$ & 0.22 \\
rs206800 & $C C+C T$ & $T T$ & $1.07(0.90$ to 1.26$)$ & 0.46 \\
rs206803 & $G G+G T$ & $T C$ & $1.12(0.93$ to 1.34$)$ & 0.22 \\
rs3769616 & $T T+T C$ & $1.06(0.85$ to 1.33$)$ & 0.58 \\
rs206804 & $A A+A G$ & $1.08(0.92$ to 1.27$)$ & 0.58 \\
\hline
\end{tabular}

Hazard ratios (95\% confidence interval) express the risk of minor allele carriers vs. non-minor allele carriers (reference), accounted for family clusters and study center modeled as random effects and adjusting for sex, age, body mass index, systolic and diastolic blood pressure, smoking and drinking, and intake of female sex hormones (all at baseline). *Significant with the false discovery rate set at 0.25 . 
$48 \mid$ Chapter 2

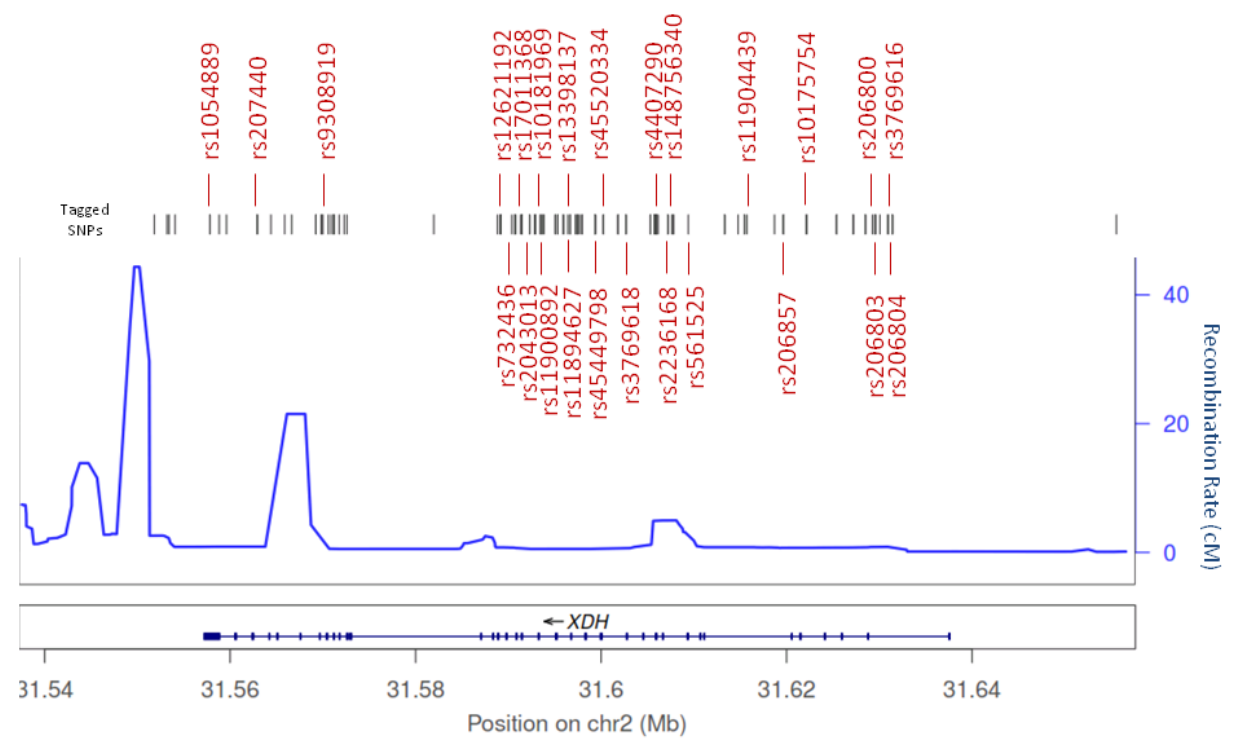

Figure S1. Plot of the XOR Gene and Flanking Regions on Chromosome 2. 



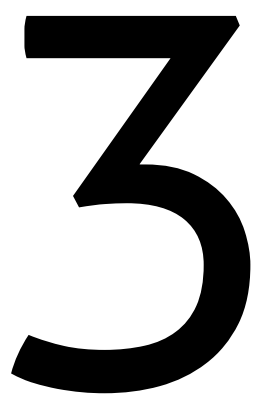

Associations of plasma uric acid and purine metabolites with blood pressure in children: the KOALA birth cohort study 


\section{ABSTRACT}

\section{Objective}

Elevated serum uric acid concentration has been associated with high blood pressure (BP) and hypertension. A putative underlying mechanism is the accumulation of reactive oxygen species when uric acid is generated by an increased enzyme activity of xanthine oxidase (XO). The aims of the present study were to investigate the associations between plasma uric acid concentration, purine metabolite ratios, as proxies for increased $X O$ activity, and SBP and DBP in school-age children.

\section{Methods}

Cross-sectional analyses were performed in 246 children $(46 \%$ boys; mean age 7.1 years) from the Dutch KOALA Birth Cohort Study. Purine metabolites were determined with ultra-performance liquid chromatography-tandem mass spectrometry. During a home visit, a nurse collected a blood sample and measured BP three times; in addition, parents measured their child's BP on three consecutive days, in the morning and evening. Generalized estimating equations were used for analyses while controlling for variables such as sex, age, body mass index, physical activity, and dietary intake.

\section{Results}

In multivariable analysis, uric acid (per SD of $38 \mathrm{mmol} / \mathrm{L}$ ) was associated with DBP z-scores [Sß 0.07; 95\% confidence interval (CI), 0.01-1.14], but not with SBP $z$-scores. Higher ratios of uric acid/xanthine (per SD of 138) (sß 0.09; $\mathrm{Cl}, 0.01-0.17$ ) and xanthine/hypoxanthine (per SD of 321) ( $S B 0.08 ; \mathrm{Cl}, 0.02-0.17$ ) were associated with higher DBP z-scores, but not with SBP z-scores.

\section{Conclusion}

In school-age children, uric acid and the ratios of uric acid/xanthine and xanthine/hypoxanthine were significantly associated with DBP z-scores. Suggesting that, both uric acid concentration and increased $\mathrm{XO}$ activity are associated with BP. 
Purine metabolites and blood pressure in children $\mid 53$

\section{INTRODUCTION}

Recent meta-analyses show that elevated serum uric acid concentration increase the risk of incident hypertension among adults, independent of traditional risk factors. ${ }^{1,2}$ Associations were significantly stronger in younger adults and tended to be larger in women, but studies among individuals younger than 18 years of age were not included in these meta-analyses. ${ }^{2}$ During the past several years, a number of studies assessed this association in older children and adolescents, and extended evidence for elevated blood pressure (BP) and hypertension among those with elevated serum uric acid concentration to this age group (summarized in Table 1 ).

Several mechanisms have been proposed that causally link uric acid with hypertension. For example, uric acid stimulates the renin-angiotensin system, leading to increased production of the vasoconstrictor angiotensin 1 . $^{3}$ Another mechanism not directly related to uric acid concentration, but rather to its production, is the generation of reactive oxygen species (ROS) during the production of uric acid. In the terminal steps of the purine catabolism, the rate-limiting enzyme xanthine oxidase $(X O)$ catalyzes the oxidation of hypoxanthine to xanthine and xanthine to uric acid (Figure 1 ). The main sources of $X O$ are the liver ${ }^{4-6}$ and intestine; ${ }^{7}$ but $X O$ can also be released into plasma and circulate to remote sites in which it binds to the surface of endothelial cells. ${ }^{8}$ When molecular oxygen acts as the electron acceptor, superoxide radical anion $\left(\mathrm{O}_{2}{ }^{\bullet-}\right)_{1}{ }^{9}$ and hydrogen peroxide $\left(\mathrm{H}_{2} \mathrm{O}_{2}\right)$ are generated as by-products of the oxidation step. These ROS inhibit endothelial nitric oxide (NO) production and consequently impair the vasodilatory reaction. ${ }^{8,10,11}$ In addition, the ROS produced by XO activity induce the adherence of leukocytes to endothelial cells, which in turn leads to more ROS generation and the initiation of proinflammatory events. ${ }^{8}$

We hypothesize that uric acid production, independent of its concentration, contributes to elevated BP and hypertension. As uric acid concentration is not an adequate marker for the production, proxies for uric acid production need to be investigated (Figure 2). As is known that $\mathrm{XO}$ is a rate-limiting enzyme, an increased $\mathrm{XO}$ activity may lead to a relative decrease in the more upstream metabolites, thus, lower hypoxanthine compared with xanthine and lower xanthine compared with uric acid concentration, resulting in higher ratios of xanthine/hypoxanthine, uric acid/xanthine, and uric acid/hypoxanthine. The latter is based on animal studies and studies examining patients with xanthine oxidoreductase deficiency, a genetic disorder called xanthinuria, who have decreased concentrations of the more upstream metabolites. ${ }^{12-15}$ 
54 Chapter 3

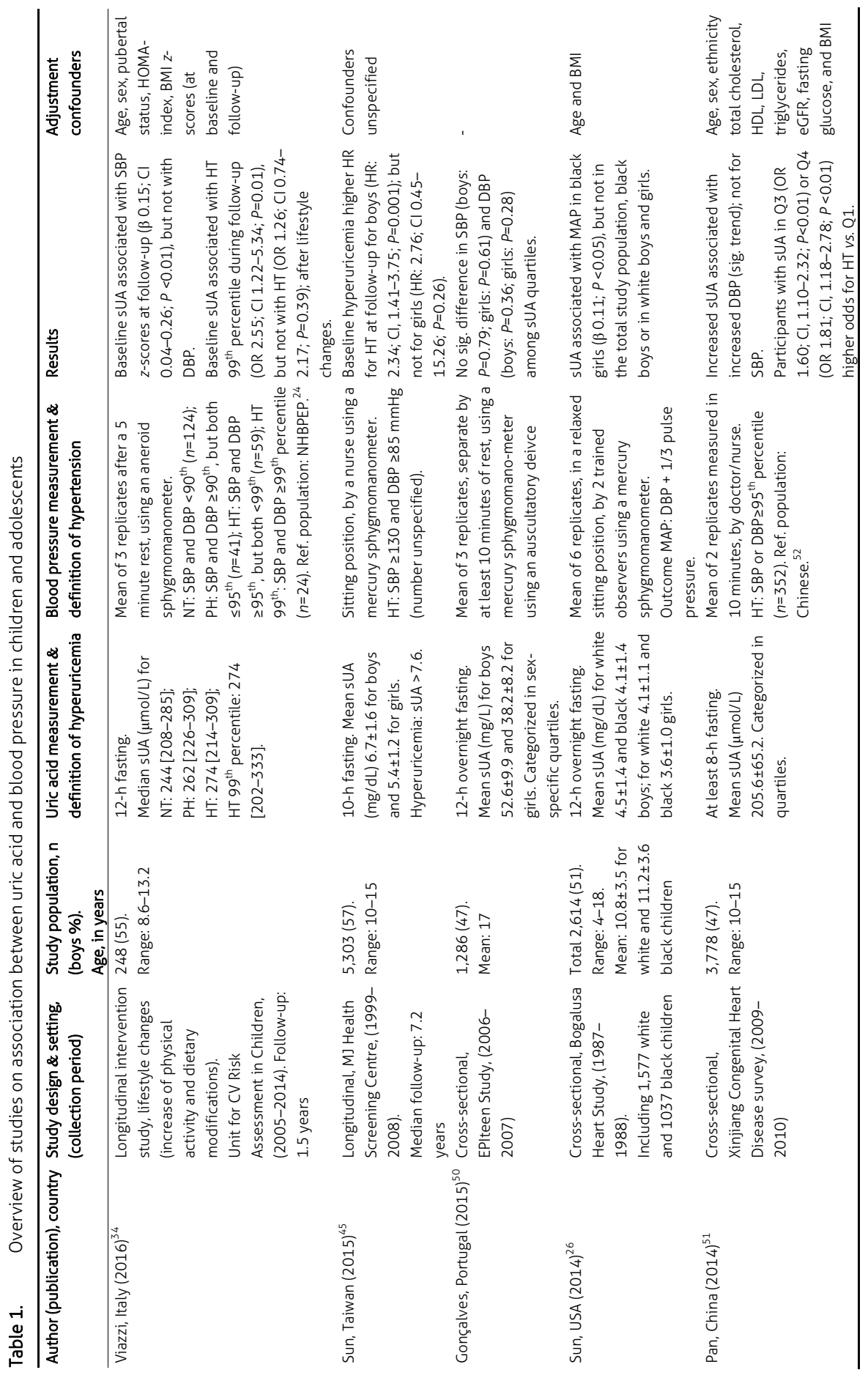


\begin{tabular}{l|l} 
& \\
Purine metabolites and blood pressure in children &
\end{tabular}

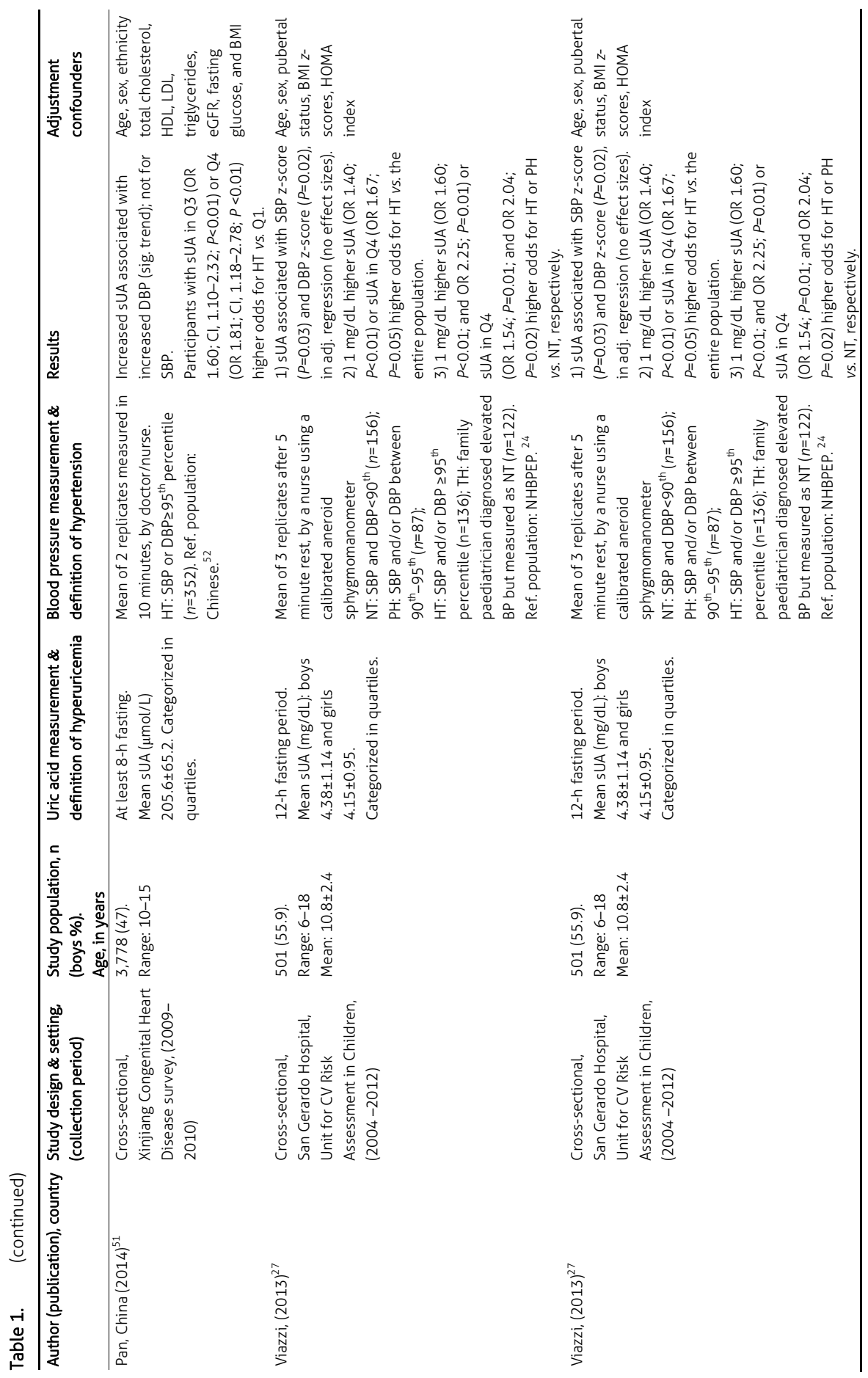


56 $\mid$ Chapter 3

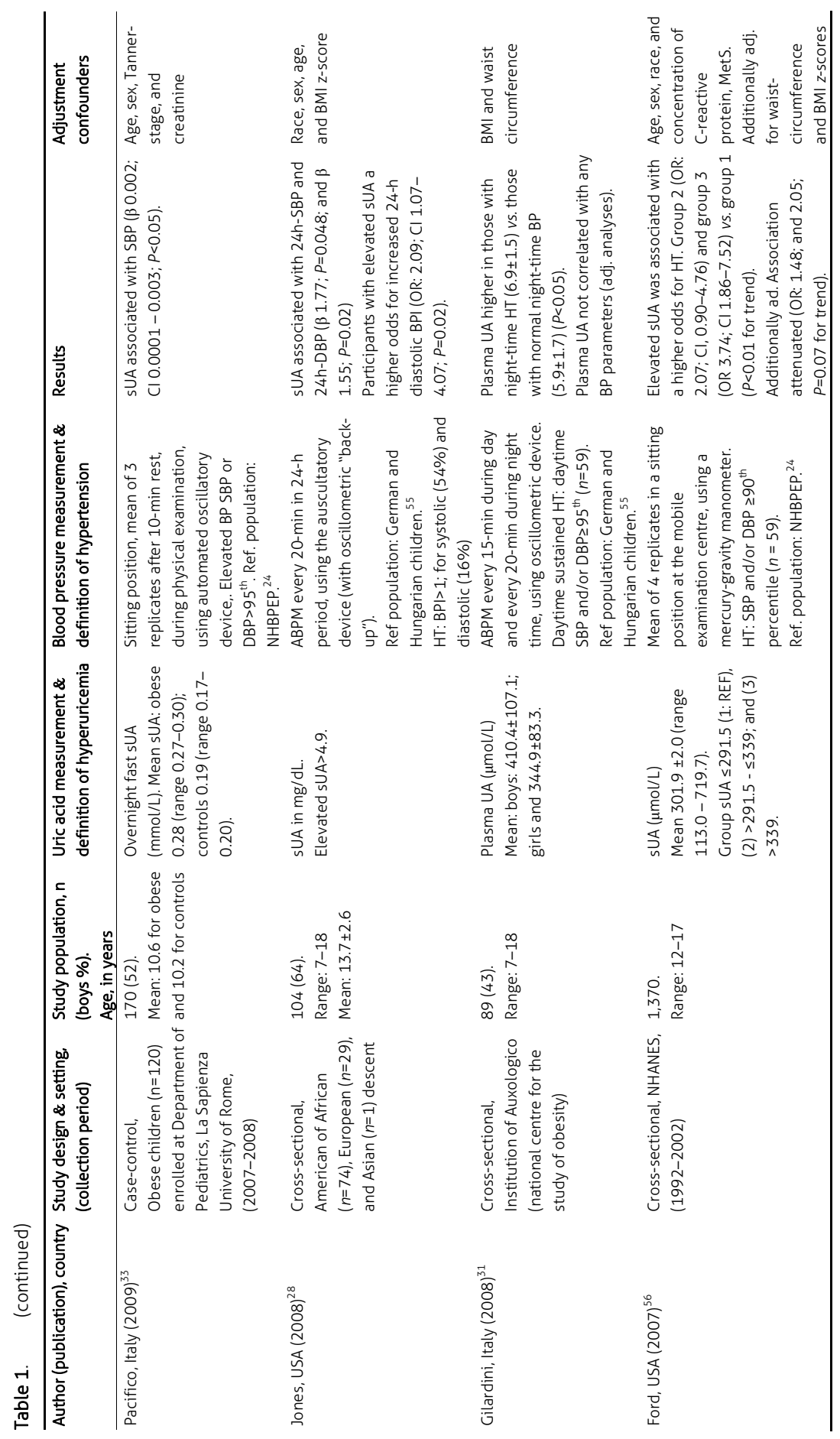


\begin{tabular}{l|l} 
Purine metabolites and blood pressure in children & 57
\end{tabular}

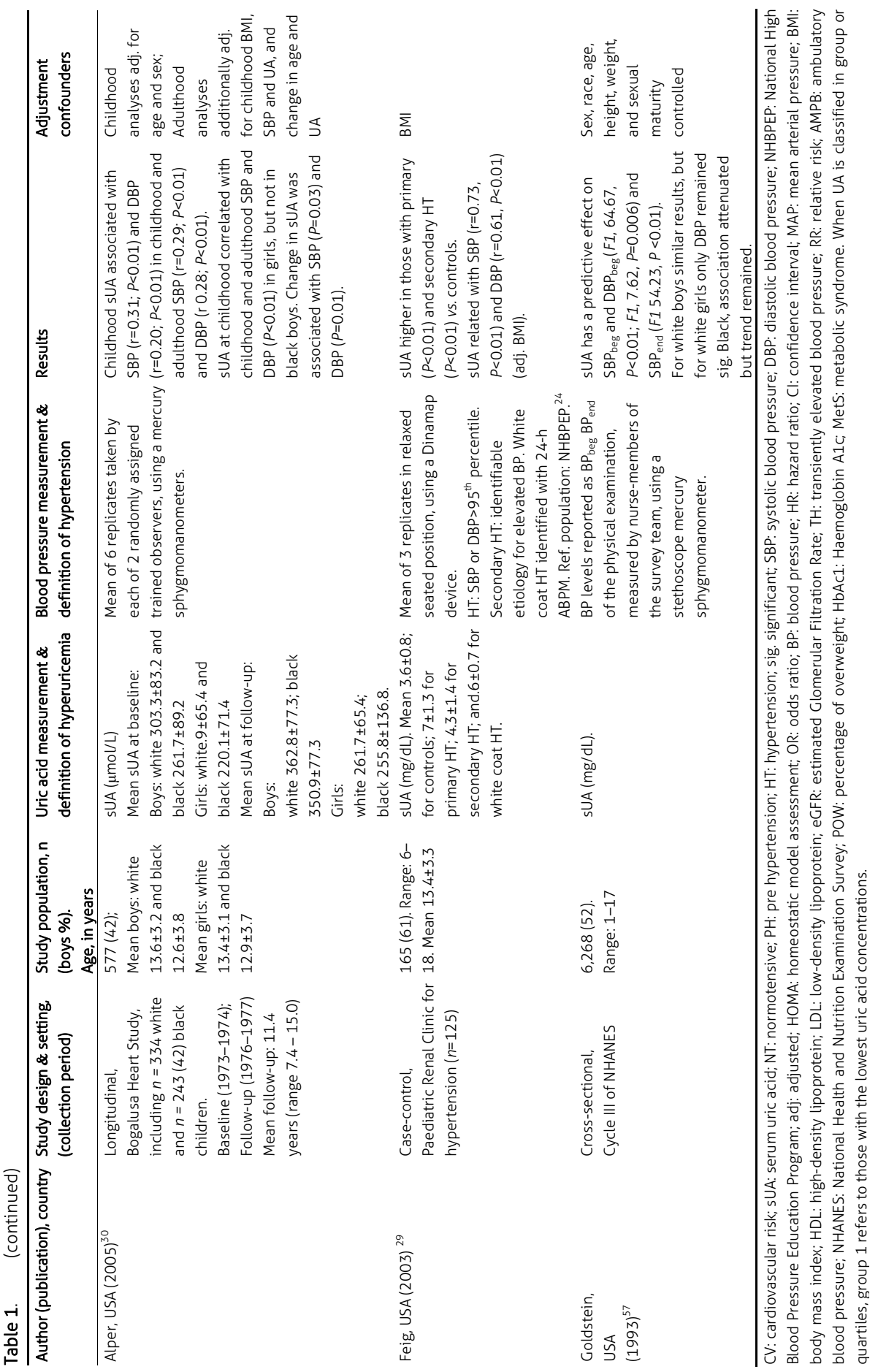




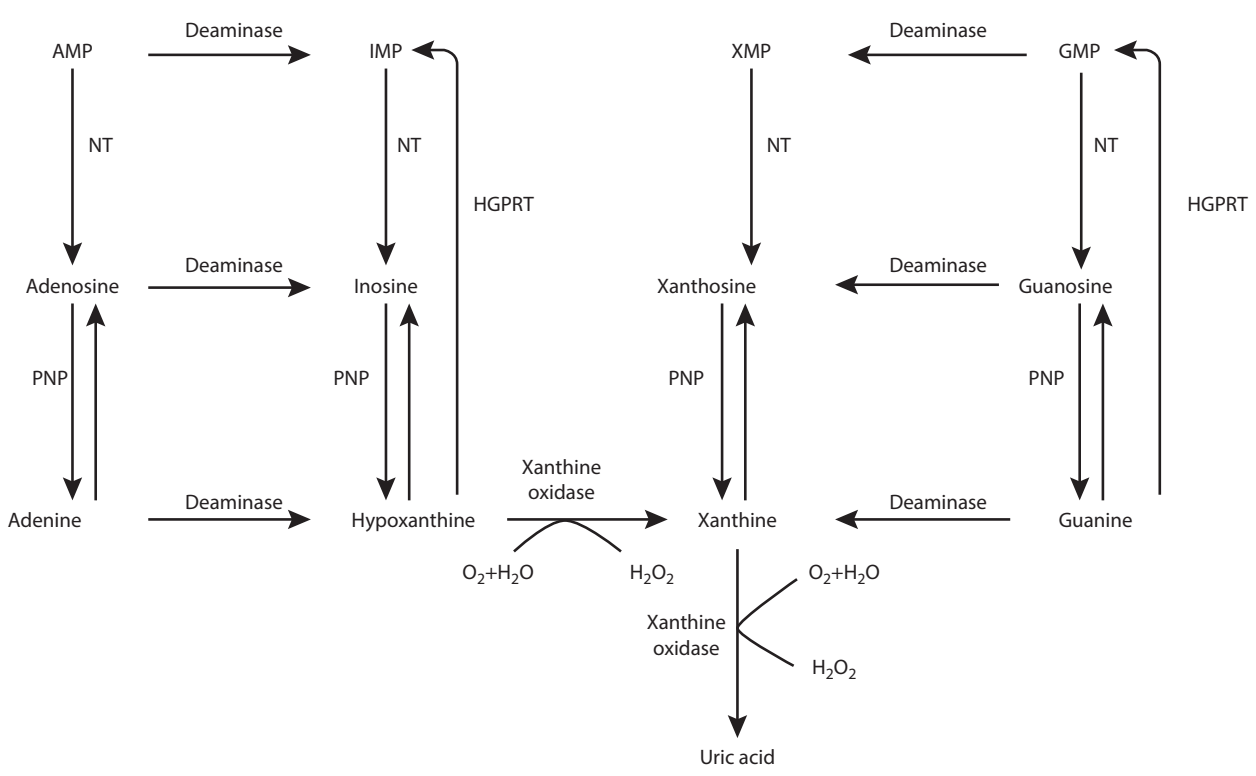

Figure 1. During the final stage of purine metabolism, xanthine oxidase $(X O)$ breaks down hypoxanthine to xanthine, and xanthine to UA, meanwhile generating reactive oxygen species (ROS). These ROS may cause oxidative stress and inactivate the vasodilator nitric oxide. AMP: adenosine monophosphate; IMP inosine monophosphate; XMP: Xanthosine monophosphate; GMP: guanosine monophosphate; NT: nucleotidase; PNP: purine nucleoside phosphorylase: HGPRT: hypoxanthine-guanine phosphoribosyltransferase.

In view of the above, the aims of the present study were to extend existing evidence on the relation between plasma uric acid concentration and BP to school-age children, and to investigate associations between purine metabolite ratios, considered as proxies for an increased $X O$ activity, and BP. Younger individuals are a desirable population in which to investigate this relationship, as common hypertension and metabolic risk factors are less likely to be present. Furthermore, uric acid may be involved in the development of hypertension before vascular damage has occurred but may have less influence on BP levels once vascular damage is permanent. ${ }^{16}$ 


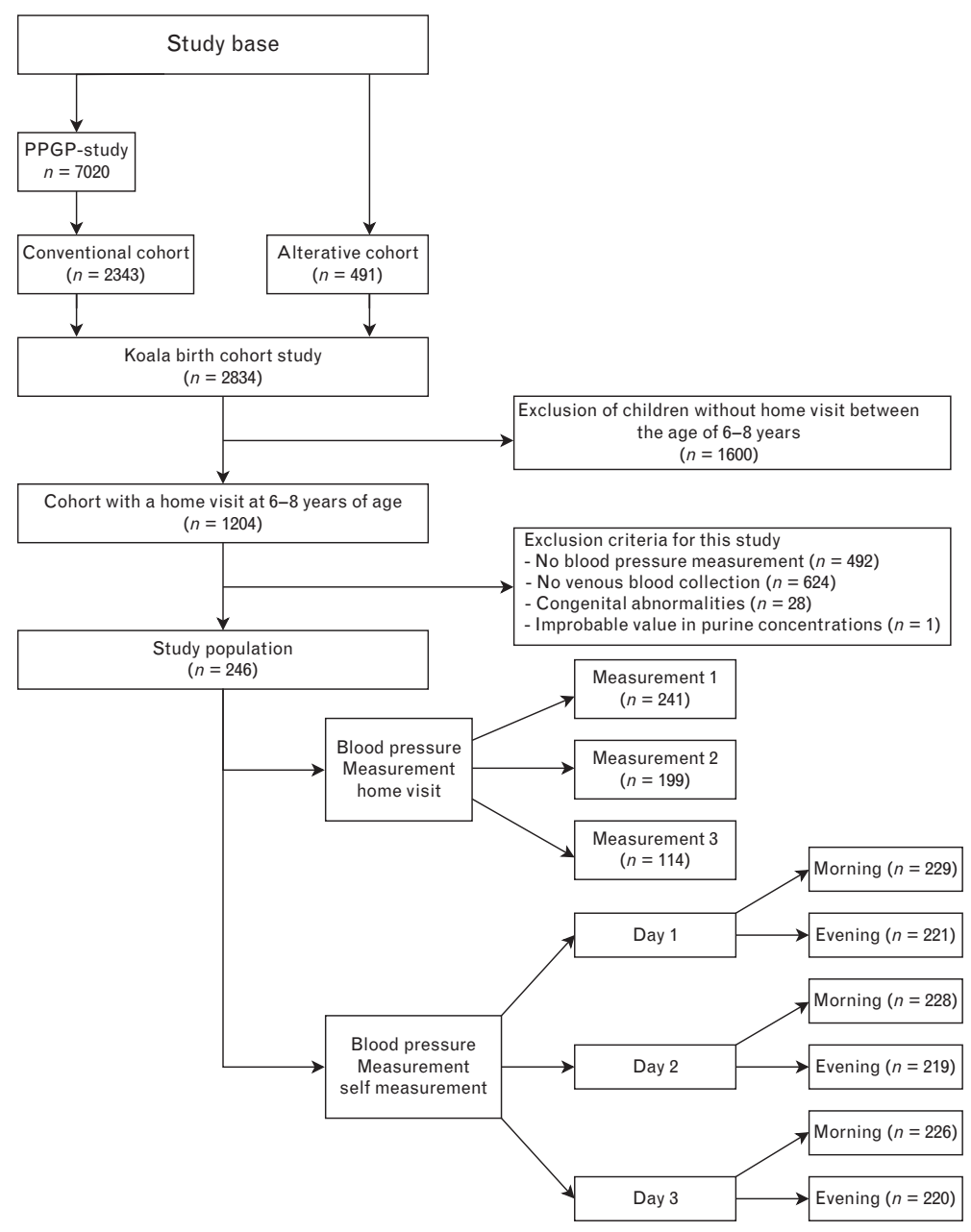

Figure 2. Flow chart of the study population. The KOALA Birth Cohort study included pregnant women with a conventional lifestyle (recruited from the on-going Pregnancy-related Pelvic Girdle Pain study) or those recruited through 'alternative' channels. Reasons for exclusion with numbers are presented.

\section{METHODS}

\section{Participants and study design}

The current analyses were conducted in the context of the KOALA Birth Cohort Study (A Dutch acronym for child, parents and health: lifestyle and genetic constitution) in The Netherlands. ${ }^{17}$ In the years 2000-2002, pregnant women were recruited at 34 weeks of gestation and followed since. The cohort originates from two recruitment groups: healthy 
$60 \mid$ Chapter 3

pregnant women with a conventional lifestyle $(n=2343)$ and pregnant women recruited through alternative channels ( $n=491$ ) (Figure 2 ). The women with a conventional lifestyle have been retrieved from an ongoing prospective cohort study on pregnancy-related pelvic girdle pain study in the Netherlands. The second recruitment group consists of pregnant women acquired through anthroposophic doctors and midwives, anthroposophic under-five clinics, posters in organic food shops, magazines for special interest groups, and Steiner schools. The latter group of women was considered to have an alternative lifestyle that could involve dietary habits (vegetarian, organic), child rearing practices, vaccination schemes, and/or use of antibiotics. All parents had signed the informed consent and the study was approved by the Medical Ethics Committee of Maastricht University Medical Centre (MUMC ${ }^{+}$).

A subgroup of 1204 parents was asked for consent for a home visit for anthropometric measurements, collection of venous blood, and a BP measurement from the child at the age of 6-7 years. This subgroup comprised participants who had home visits for blood collection from the mother during pregnancy and/or the child at age 2 years, and who were still active participants. Venous blood samples were obtained from 600 children and BP measurements from 712 children. Exclusion criteria for the current study were children with congenital heart disease, severe mental disability and/or severe autism, twins, and improbable values in purine concentrations. A total of 246 children that met the criteria were included in the present study (Figure 2).

Information on potential confounders was prospectively collected by parent-completed questionnaires during pregnancy (weeks 14 and 34) and at ages 3, 7, 12, and 24 months, 4-5 years, and 6-7 years of the child. Dietary information was collected using a food frequency questionnaire (FFO) covering a period of 4 weeks at the age of 4 years. The FFO was specifically developed to assess childhood energy intake and validated by the doubly labelled water method. ${ }^{18}$ Details on collection of dietary information are described elsewhere. ${ }^{19}$ During the home visit at 6-7 years, the child's body weight and height were measured. BMI (weight/height ${ }^{2}, \mathrm{~kg} / \mathrm{m}^{2}$ ) was standardized by recoding it into age-specific and sex-specific BMI z-scores using data from the Dutch reference population. ${ }^{20}$ For descriptive purposes, children were classified into normal weight (BMI $z$-score $<1.04$, corresponding to the $85^{\text {th }}$ percentile), overweight (BMI z-score between $\geq 1.04$ and $<1.65$, corresponding to the $85^{\text {th }}$ and $95^{\text {th }}$ percentile), and obesity (BMI z-score between $\geq 1.65$, corresponding to the $95^{\text {th }}$ percentile). ${ }^{21}$

\section{Purine metabolite determination}

Venous blood samples were collected in EDTA tubes during the home visit to assess uric acid, xanthine, and hypoxanthine concentrations in plasma. For ethical reasons children were not asked to fast overnight; however, $1.5 \mathrm{~h}$ prior to and during the home visit, the children were not allowed to eat or drink, resulting in a mean fast time of $2.2 \mathrm{~h}$ (SD $1.2 \mathrm{~h}$ ). After collection, the samples were stored at $-20^{\circ} \mathrm{C}$ and thawed in a water bath at $37^{\circ} \mathrm{C}$ prior to analysis and homogenated by vortex mixing. A volume of $30 \mathrm{ml}$ plasma was 
mixed with $30 \mathrm{~mL}$ of internal standards solution, and while vortex mixing $500 \mathrm{ml}$ of acetonitrile was added and vortex mixed for another $10 \mathrm{~s}$. After centrifugation, the supernatant was transferred to a glass sample vial and evaporated to dryness under nitrogen gas at $45^{\circ} \mathrm{C}$ for $\pm 15 \mathrm{~min}$. The sample was then taken up in $150 \mathrm{ml} 50 \mathrm{mmol} / \mathrm{l}$ ammoniumformiate $\mathrm{pH}$ 4.00. The purine metabolites were determined with ultraperformance liquid chromatography-tandem mass spectrometry. ${ }^{22}$ The ratios of uric acid to xanthine [uric acid (mmol/L)/xanthine $(\mathrm{mmol} / \mathrm{L})$ ], uric acid to hypoxanthine [uric acid $(\mathrm{mmol} / \mathrm{L}) /$ hypoxanthine $(\mathrm{mmol} / \mathrm{L})]$, and xanthine to hypoxanthine [xanthine $(\mathrm{mmol} / \mathrm{L}) /$ hypoxanthine $(\mathrm{mmol} / \mathrm{L})]$ were calculated.

\section{Blood pressure measurement}

Mean arterial BP was measured during the home visit, using a validated Omron 705IT oscillometric automated BP monitor (Omron Healthcare Europe BV, Hoofddorp, The Netherlands). ${ }^{23}$ The device automatically provided an estimated SBP and DBP. Up to three measurements were performed by a trained nurse after a 5-min rest, whereas the child was sitting and not allowed to talk or move. BP was measured up to three times on the upper left arm while it was resting on a table. Afterwards, parents were asked to measure their child's BP on three consecutive days, in the morning and in the evening. The parents were instructed by the trained nurse and the same protocol was used. SBP and DBP percentiles and z-scores were calculated according to the nomograms recommended by the National High Blood Pressure Education Program on High Blood Pressure in Children and Adolescents (in the Fourth Report). ${ }^{24}$ As BP was measured with an oscillometric device during a single study visit, a formal diagnosis of hypertension is not possible. Therefore, participants were characterized as having 'normal blood pressure' if the mean (measurement performed by the nurse) of SBP and DBP were both less than $95^{\text {th }}$ percentile and 'elevated blood pressure' if the mean SBP or DBP percentile was at least $95^{\text {th }}$ percentile.

\section{Statistical analysis}

The characteristics of the participants are given as numbers and proportions for categorical variables and as mean (SD) or median (interquartile range) values for continuous variables with a normal or nonnormal distribution, respectively. Missing values were classified as unknown for categorical variables (maternal smoking during pregnancy $n=3$; passive smoking during pregnancy $n=6$ ). Participants with missing values on the FFO $(n=11)$ were excluded for the analyses in the third model. Data analysis was performed using SPSS version 20.0 (SPSS Inc., Chicago, Illinois, USA).

Uric acid concentration differs between men and women; therefore, purine values and their ratios were compared between boys and girls with the independent $t$ test or MannWhitney test for values with a normal or nonnormal distribution data, respectively. ${ }^{25}$ Standardized SBP and DBP z-scores were compared across the three different conditions (home visit measurements versus self-measurement and self-measurements in the 
$62 \mid$ Chapter 3

morning versus self-measurements in the evening), using the dependent $t$ test, respectively.

Generalized estimating equation (GEE) models with unstructured correlation structure were used for analysis of the repeated BP measurements [studied as standardized $z$-scores (adj. for age, sex, and height z-scores)]. Up to nine measurements were available, taken under three different conditions. The BP measurement condition (as the index variable) and recruitment group (conventional; alternative) as confounder were included in the first model. The analyses included only one single purine metabolite or ratio at a time. In the second model, the following confounders were additionally included: age, infant sex (male, female), and BMI z-scores. In the third model, potential confounders that changed the regression coefficient of uric acid and SBP or DBP by more than $10 \%$ were added to the GEE model. The following variables met this criterion: place and mode of delivery (vaginal delivery at home, vaginal delivery in hospital, artificial delivery in hospital, and caesarean section in hospital), maternal smoking during pregnancy (no, yes, and unknown), passive smoking during pregnancy ( $<1 \mathrm{~h}$ a week, $>1 \mathrm{~h}$ a week, and unknown), total physical activity at 4-5 years of age (h/week), energy intake (kJ/day), protein intake (\%), carbohydrate intake (\%), and fibre intake (g/day). By adding dietary variables as confounder in the analyses, we attempt to control for dietary factors that potentially affect BP. The following variables did not meet the criterion and were therefore not included in the third model: prematurity (born before 37 weeks of gestation), maternal pre-pregnancy BMI $\left(\mathrm{kg} / \mathrm{m}^{2}\right)$, alcohol consumption during late pregnancy (number of consumptions), education level of the mother (lower education, middle education, and high education), birth weight (in grams), duration of breastfeeding (months), and fasting time before blood collection (h).

Finally, we tested whether the association between uric acid concentrations with BP z-scores was modified by sex or BP measurement condition by including an interaction term in the fully adjusted GEE model. The interaction terms were nonsignificant ( $P$ value $>0.05$ ); therefore, the analyses were not stratified by sex or BP measurement condition.

\section{RESULTS}

The characteristics of the KOALA cohort $(n=2834)$ and the study population are shown in Table 2.

The study population consisted of 246 children, who were on average ages $7.1 \pm 0.4$ years and of whom 114 (46.3\%) were boys. In general, the two populations were similar, except that the study population consisted of less mothers who smoked during pregnancy (active and passive) and more children were delivered naturally at home than in the entire KOALA cohort. 
Table 2. Baseline characteristics of the KOALA Birth Cohort Study and the study population

\begin{tabular}{|c|c|c|c|}
\hline & & $\begin{array}{c}\text { KOALA Birth Cohort } \\
\text { Study } \\
(n=2834) \\
\end{array}$ & $\begin{array}{l}\text { Study population } \\
\qquad(n=246)\end{array}$ \\
\hline Gender, boy n (\%) & & $1543(50.9)$ & $114(46.3)$ \\
\hline Recruitment group, conventional n (\%) & & $2343(82.7)$ & $207(84.1)$ \\
\hline Age during home visit (years), mean \pm SD & & - & $7.05 \pm 0.41$ \\
\hline BMI z-score during home visit, mean \pm SD & & - & $-0.24 \pm 0.87$ \\
\hline Normal weight, n (\%) & & & $227(92.3)$ \\
\hline Overweight, n (\%) & & & $16(6.5)$ \\
\hline Obese, $n(\%)$ & & & $3(1.2)$ \\
\hline Height z-scored, mean \pm SD & & & $0.50 \pm 0.88$ \\
\hline Birth weight (grams), mean \pm SD & & $3504 \pm 512$ & $3551 \pm 438$ \\
\hline \multirow[t]{4}{*}{ Maternal education, ${ }^{\mathrm{a}} \mathrm{n}(\%)$} & Low & $289(9.5)$ & $20(8.1)$ \\
\hline & Middle & $1060(34.9)$ & $89(36.2)$ \\
\hline & High & $1341(44.2)$ & $123(50.0)$ \\
\hline & Unknown & $343(11.3)$ & $14(5.7)$ \\
\hline Maternal pre-pregnancy BMI, mean \pm SD & & $23.5 \pm 4.5$ & $23.5 \pm 4.1$ \\
\hline \multirow[t]{2}{*}{ Maternal smoking during pregnancy, $\mathrm{n}(\%)$} & yes & $222(7.3)$ & $11(4.5)$ \\
\hline & unknown & $209(6.9)$ & $3(1.2)$ \\
\hline \multirow{2}{*}{$\begin{array}{l}\text { Maternal passive smoking during pregnancy (>1h week), } \\
\mathrm{n}(\%)\end{array}$} & yes & $448(14.8)$ & $23(9.3)$ \\
\hline & unknown & $255(8.4)$ & $6(2.4)$ \\
\hline $\begin{array}{l}\text { Alcohol consumption during pregnancy (number of } \\
\text { glasses), mean } \pm \text { SD }\end{array}$ & & $0.3 \pm 0.9$ & $0.2 \pm 0.7$ \\
\hline Duration of pregnancy, & Mean \pm SD & $39.8 \pm 5.0$ & $39.5 \pm 1.1$ \\
\hline \multirow[t]{2}{*}{$n(\%)$} & $<37$ weeks & $93(3.1)$ & $0(0)$ \\
\hline & Unknown & $202(6.7)$ & $1(0.4)$ \\
\hline \multirow[t]{6}{*}{ Place and mode of delivery, $\mathrm{b}(\%)$} & Natural delivery at home & $1187(39.1)$ & $113(45.9)$ \\
\hline & Natural delivery in hospital & $924(30.5)$ & $81(32.9)$ \\
\hline & Artificial delivery at home & $1(0.0)$ & $0(0.0)$ \\
\hline & Artificial delivery in hospital & $223(7.4)$ & $23(9.3)$ \\
\hline & Caesarean section in hospital & $311(10.3)$ & $28(11.4)$ \\
\hline & Unknown & $381(12.6)$ & $1(0.4)$ \\
\hline Duration of breastfeeding (months), mean \pm SD & & $4.91 \pm 4.55$ & $5.5 \pm 4.2$ \\
\hline Total physical activity (hours/week), mean \pm SD & & $9.4 \pm 4.5$ & $9.6 \pm 4.7$ \\
\hline \multirow[t]{5}{*}{ Dietary factors of the child at age four, mean $\pm S D^{C}$} & Total energy intake, kilojoules & $6172 \pm 1286$ & $6086 \pm 1331$ \\
\hline & Energy percentage from fat & $29.6 \pm 4.2$ & $29.6 \pm 3.9$ \\
\hline & Energy percentage from protein & $14.6 \pm 2.1$ & $14.8 \pm 2.1$ \\
\hline & $\begin{array}{l}\text { Energy percentage from } \\
\text { carbohydrate }\end{array}$ & $55.8 \pm 5.0$ & $55.5 \pm 4.7$ \\
\hline & Fibre, grams & $2.5 \pm 0.5$ & $2.5 \pm 0.5$ \\
\hline
\end{tabular}

\footnotetext{
* Numbers do not always add up to the total because of missing data. Abbreviations: SD, standard deviation; BMI, body mass index. ${ }^{a}$ Low: primary school, preparatory vocational or lower general secondary school, Middle: vocational, higher general secondary or pre-university education, High: higher vocational or academic education. ${ }^{b}$ Artificial delivery: Induced labour, as when started with drugs or medical devices. c Missing data for 13 participants on variables total physical activity and dietary factors.
}

Data on purine metabolite concentrations and their ratios are shown in Table 3 . The mean plasma uric acid concentration was slightly higher for boys $[203.9 \mathrm{mmol} / \mathrm{L}$ (SD 39.6)] than for girls [201.8 mmol/L (SD 36.2)] but did not differ significantly $(P=0.67)$. 
64 Chapter 3

Median hypoxanthine [boys: $2.4 \mathrm{mmol} / \mathrm{L}$ (IOQR 2.7); girls $2.5 \mathrm{mmol} / \mathrm{L}$ (IOQR 3.5)] and mean xanthine concentrations [boys: $0.5 \mathrm{mmol} / \mathrm{L}$ (SD 0.20); girls: $0.5 \mathrm{mmol} / \mathrm{L}$ (SD 0.15)] and the different ratios did not differ substantially by sex.

Table 3. Plasma concentrations of uric acid, xanthine, and hypoxanthine and their ratios

\begin{tabular}{llccc}
\hline & & $\begin{array}{c}\text { Total population } \\
(n=246)\end{array}$ & $\begin{array}{c}\text { Boys } \\
(n=114)\end{array}$ & $\begin{array}{c}\text { Girls } \\
(n=132)\end{array}$ \\
\hline Purine metabolite & & & & \\
Uric acid $(\mu \mathrm{mol} / \mathrm{L})$ & Mean \pm SD & $202.8 \pm 37.7$ & $203.9 \pm 39.6$ & $201.8 \pm 36.2$ \\
Xanthine $(\mu \mathrm{mol} / \mathrm{L})$ & Mean \pm SD & $0.53 \pm 0.17$ & $0.53 \pm 0.20$ & $0.53 \pm 0.15$ \\
Hypoxanthine $(\mu \mathrm{mol} / \mathrm{L})$ & Median $(\mathrm{IOR})$ & $2.5(3.2)$ & $2.4(2.7)$ & $2.5(3.5)$ \\
Ratio & & & & \\
Uric acid / xanthine & Mean \pm SD & $417.2 \pm 138.4$ & $425.0 \pm 154.1$ & $410.5 \pm 123.4$ \\
Uric acid / hypoxanthine & Median (IOR) & $83.9(94.5)$ & $86.7(102.5)$ & $81.6(86.5)$ \\
Xanthine / hypoxanthine & Median (IOR) & $4.7(4.8)$ & $4.7(4.6)$ & $4.6(4.8)$ \\
\hline
\end{tabular}

Abbreviations: SD: standard deviation; IQR: interquartile range

In Table 4, BP data are presented. Elevated BP was found in 64 (26\%) of the children. The correlation among the self-measurement ranged from 0.20 up to 0.50 . BP z-scores were higher when measurements were performed by the parents than these performed by the nurse $(P$-value $<0.001)$. Furthermore, measurements performed in the evening were higher than these performed in the morning for SBP $z$-score $(P=0.001)$, but not for DBP z-score $(P=0.96)$.

Table 4. Blood pressure levels measured by a trained nurse during the home visit and by the parents in the morning and evening

\begin{tabular}{lccc}
\hline & & Systolic BP $z$-scores & Diastolic BP $z$-scores \\
\hline Home visit $^{\text {a }}$ & & & \\
& Mean \pm SD & $0.84 \pm 0.69$ & $0.49 \pm 0.86$ \\
Self-measurement $^{\text {Morning }}{ }^{b}$ & Mean \pm SD & $0.78 \pm 0.94$ & $0.49 \pm 0.88$ \\
Evening $^{c}$ & Mean \pm SD & $0.88 \pm 0.94$ & $0.49 \pm 0.85$ \\
\hline
\end{tabular}

BP: blood pressure. Home visit: BP is measured by a trained nurse, the mean of three replicates. Self-measurement: $\mathrm{BP}$ is measured by the parents on three consecutive days, in the morning and evening. Missing for each condition: home visit: $n=5$ (boys $n=1$; girls $n=4$; self-measurement: morning $n=16$ (boys $n=5$; girls $n=11$ ) and evening $n=23$ (boys $n=8$; girls $n=15$ ).

\section{Purine metabolite concentrations and blood pressure}

Multivariable analysis showed that a $1 \mathrm{SD}(38 \mathrm{mmol} / \mathrm{L})$ higher plasma uric acid concentration was associated with a higher DBP z-scores, although statistical significance was only reached in the fully adjusted model [adj. sß 0.07; 95\% confidence interval (CI), 0.01-0.14]. No association was found with SBP z-scores (Table 5). Plasma xanthine and hypoxanthine concentrations were not associated with SBP z-scores or DBP z-scores, regardless of adjustment for potential confounders. Although the association 
between plasma hypoxanthine with DBP z-scores was close to significance (adj. sß 0.06; $\mathrm{Cl},-0.01$ to $0.13 ; P=0.10$ ). An increase of $0.10 \mathrm{DBP} z$-score represents a difference of $0.93 \mathrm{mmHg}$ for a 6-year-old boy with a height z-score of 0.00 .

Table 5. Association between purine metabolite and their ratios with blood pressure z-scores in children.

\begin{tabular}{|c|c|c|c|c|c|c|c|}
\hline & & $\begin{array}{c}\text { Model } 1 \mathrm{~s} \beta(95 \% \\
(\mathrm{Cl})^{\mathrm{a}}\end{array}$ & $P$ & $\begin{array}{c}\text { Model } 2 \mathrm{~s} \beta(95 \% \\
(\mathrm{Cl})^{\mathrm{b}}\end{array}$ & $P$ & $\begin{array}{c}\text { Model } 3 \text { s } \beta(95 \% \\
(\mathrm{Cl})^{\mathrm{c}}\end{array}$ & $P$ \\
\hline \multicolumn{8}{|l|}{ Purine metabolite } \\
\hline \multirow[t]{2}{*}{ Uric acid } & SBP & $\begin{array}{c}0.000 \\
(-0.08 \text { to } 0.07)\end{array}$ & 0.99 & $\begin{array}{c}0.01 \\
(-0.07 \text { to } 0.08)\end{array}$ & 0.90 & $\begin{array}{c}-0.02 \\
(-0.10 \text { to } 0.06)\end{array}$ & 0.70 \\
\hline & DBP & $\begin{array}{c}0.04 \\
(-0.02 \text { to } 0.11)\end{array}$ & 0.17 & $\begin{array}{c}0.05 \\
(-0.01 \text { to } 0.12)\end{array}$ & 0.08 & $\begin{array}{c}0.07 \\
(0.01 \text { to } 1.14)\end{array}$ & 0.03 \\
\hline \multirow[t]{2}{*}{ Xanthine } & SBP & $\begin{array}{c}-0.001 \\
(-0.08 \text { to } 0.08)\end{array}$ & 0.99 & $\begin{array}{c}0.002 \\
(-0.08 \text { to } 0.08)\end{array}$ & 0.97 & $\begin{array}{c}-0.03 \\
(-0.10 \text { to } 0.05)\end{array}$ & 0.49 \\
\hline & DBP & $\begin{array}{c}-0.03 \\
(-0.10 \text { to } 0.05)\end{array}$ & 0.61 & $\begin{array}{c}-0.02 \\
(-0.10 \text { to } 0.05)\end{array}$ & 0.54 & $\begin{array}{c}-0.04 \\
(-1.11 \text { to } 0.04)\end{array}$ & 0.35 \\
\hline \multirow[t]{2}{*}{ Hypoxanthine } & SBP & $\begin{array}{c}0.01 \\
(-0.01 \text { to } 0.10)\end{array}$ & 0.76 & $\begin{array}{c}0.01 \\
(-0.07 \text { to } 0.09)\end{array}$ & 0.78 & $\begin{array}{c}0.01 \\
(-0.08 \text { to } 0.10)\end{array}$ & 0.82 \\
\hline & DBP & $\begin{array}{c}0.05 \\
(-0.02 \text { to } 0.12)\end{array}$ & 0.13 & $\begin{array}{c}0.06 \\
(-0.01 \text { to } 0.12)\end{array}$ & 0.11 & $\begin{array}{c}0.06 \\
(-0.01 \text { to } 0.13)\end{array}$ & 0.10 \\
\hline \multicolumn{8}{|l|}{ Ratio } \\
\hline \multirow[t]{2}{*}{ Ratio uric acid / xanthine } & SBP & $\begin{array}{c}-0.01 \\
(-0.10 \text { to } 0.08)\end{array}$ & 0.81 & $\begin{array}{c}-0.01 \\
(-0.09 \text { to } 0.05)\end{array}$ & 0.83 & $\begin{array}{c}0.003 \\
(-0.08 \text { to } 0.08)\end{array}$ & 0.93 \\
\hline & DBP & $\begin{array}{c}0.06 \\
(-0.02 \text { to } 0.14)\end{array}$ & 0.15 & $\begin{array}{c}0.07 \\
(-0.01 \text { to } 0.15)\end{array}$ & 0.10 & $\begin{array}{c}0.09 \\
(0.01 \text { to } 0.17)\end{array}$ & 0.03 \\
\hline \multirow[t]{2}{*}{$\begin{array}{l}\text { Ratio uric acid / } \\
\text { hypoxanthine }\end{array}$} & SBP & $\begin{array}{c}-0.002 \\
(-0.04 \text { to } 0.04)\end{array}$ & 0.93 & $\begin{array}{c}-0.01 \\
(-0.05 \text { to } 0.04)\end{array}$ & 0.28 & $\begin{array}{c}-0.003 \\
(-0.06 \text { to } 0.06)\end{array}$ & 0.91 \\
\hline & DBP & $\begin{array}{c}-0.03 \\
(-0.05 \text { to }-0.00)\end{array}$ & 0.04 & $\begin{array}{c}-0.03 \\
(-0.06 \text { to } 0.01)\end{array}$ & 0.11 & $\begin{array}{c}-0.03 \\
(-0.08 \text { to } 0.01)\end{array}$ & 0.17 \\
\hline \multirow[t]{2}{*}{$\begin{array}{l}\text { Ratio xanthine / } \\
\text { hypoxanthine }\end{array}$} & SBP & $\begin{array}{c}0.01 \\
(-0.07 \text { to } 0.10)\end{array}$ & 0.74 & $\begin{array}{c}0.01 \\
(-0.07 \text { to } 0.09)\end{array}$ & 0.79 & $\begin{array}{c}0.03 \\
(-0.06 \text { to } 0.12)\end{array}$ & 0.50 \\
\hline & DBP & $\begin{array}{c}0.06 \\
(-0.00 \text { to } 0.12)\end{array}$ & 0.06 & $\begin{array}{c}0.06 \\
(-0.00 \text { to } 0.12)\end{array}$ & 0.06 & $\begin{array}{c}0.08 \\
(0.02 \text { to } 0.14)\end{array}$ & 0.01 \\
\hline
\end{tabular}

$\mathrm{Cl}$, confidence interval; BMI, body mass index; SBP: systolic blood pressure; DBP: diastolic blood pressure. ${ }^{a}$ Adjusted for recruitment group (conventional; alternative). ${ }^{b}$ Additionally adjusted for age, sex, and BMI z-scores. ${ }^{c}$ Additionally adjusted for place and mode of delivery, maternal smoking during pregnancy (active and passive), total physical activity, and nutrition intake at 4 years of age (total energy intake (kcal), energy from carbohydrates $(\%)$ and energy from protein (\%) and fibre intake (grams). Analyses included 232 children, due to missing values on the FFO and for total physical activity $(n=13)$. The purine metabolites and ratios expressed as standard deviations (uric acid $37.7 \mu \mathrm{mol} / \mathrm{L}$; xanthine $0.17 \mu \mathrm{mol} / \mathrm{L}$; hypoxanthine $6.9 \mu \mathrm{mol} / \mathrm{L}$ and the ratios uric acid/xanthine 138.4; uric acid/hypoxanthine 321.4, and xanthine / hypoxanthine 8.8). Outcome presented as standardized blood pressure $z$-scores (adj. for age, gender, and height z-scores; ref. population: NHBPEP. ${ }^{24}$

\section{Purine metabolite ratios and blood pressure}

The ratios uric acid/xanthine and xanthine/hypoxanthine were associated with DBP z-scores, although statistical significance was only reached in the fully adjusted model (adj. sB 0.09; $\mathrm{Cl}, 0.01-0.17$ ) and (adj. SB 0.08; $\mathrm{Cl}, 0.02-0.14$ ), respectively. None of the ratios was significantly associated with SBP z-scores. The ratio uric acid/hypoxanthine 
$66 \mid$ Chapter 3

was associated with DBP z-scores in the crude model but lost significance after adjustment for potential confounders.

\section{DISCUSSION}

The present study showed that, in school-age children, elevated plasma uric acid concentration was associated with higher DBP z-scores. Furthermore, elevated ratios of uric acid/xanthine and xanthine/hypoxanthine, considered as proxies for increased $X O$ activity, were associated with higher DBP z-scores. Our findings support the hypothesis that next to an elevated uric acid concentration, an increased $X O$ activity is associated with elevated BP.

Although an association between serum uric acid concentration and BP has been described before in older children and adolescents (summarized in Table 1), we were the first to show an association in school-age children at a mean age of 7 years. The studies performed so far mainly studied older children (with a mean age of 10 years or higher) and adolescents. ${ }^{26-30}$ Moreover, we found an effect in a relatively healthy group of children, with respect to both the participants' uric acid concentration [hyperuricaemia (plasma uric acid concentration $>5.0 \mathrm{mg} / \mathrm{dL}$ ) $n=0]$, whereas other studies often investigate children from clinical cohorts, who have a relatively high risk of cardiovascular diseases. ${ }^{28,29,31-34}$

To the best of our knowledge, we were the first to study the association between BP and several ratios of purine metabolites as proxies for an increased $\mathrm{XO}$ activity in the vasculature. We showed that higher uric acid/xanthine and xanthine/hypoxanthine ratios were associated with higher DBP z-scores. Our hypothesis is based on studies examining the effect of a deficiency in $X O$ on purine metabolite concentrations; ${ }^{13-15}$ however, whether an opposite effect in purine metabolite concentrations occurs by an increased $X O$ activity is unknown. Further investigation is required to examine whether increased $X O$ activity leads to a relative increase in the more downstream metabolites and that this will not be compensated by alterations in urinary excretion of uric acid or xanthine, or the degradation of hypoxanthine to inosine monophosphate by hypoxanthine-guanine phosphoribosyltransferase. ${ }^{15}$

In the present study, we found an association with DBP z-scores but not with SBP z-scores. Diastolic pressure and mean arterial pressure, the steady components of BP, reflect peripheral vascular resistance. Particularly in younger individuals ( $<50$ years of age), DBP is the main predictor of cardiovascular risk. Later in life SBP and pulse pressure, the pulsatile components of BP become the main predictors. ${ }^{35-37}$ As previously described, there are several plausible mechanisms linking uric acid to vascular resistance. ${ }^{3}$ For example, uric acid in the circulation activates angiotensin II, thereby stimulating the nicotinamide adenine dinucleotide (phosphate) (reduced form) (NAD $(P) H)$ oxidase that is the main source of ROS in the vasculature. ROS reduces the bioavailability of vasodilator NO, leading to vascular resistance. In turn, ROS increases the formation of 
peroxynitrite, which can increase endothelial NO synthase uncoupling resulting in even more ROS formation. ${ }^{38,39}$ Moreover, Landmesser et al. found that angiotensin II substantially increases endothelial $X O$ activation in cultured endothelial cells, subsequent to $\mathrm{NAD}(\mathrm{P}) \mathrm{H}$ oxidase activation, leading to even more ROS formation. ${ }^{40}$ Moreover, another mechanism not directly related to uric acid concentration, but rather to its production, is the accumulation of ROS during the degradation of purines by increased $X O$ activity.

The magnitude of the BP change found in our study is relatively small; an increase of 1 $\mathrm{SD}$ in uric acid concentration or uric acid/xanthine and xanthine/hypoxanthine ratios was associated with an increase of approximately 0.08 higher DBP z-score. This represents an increase in DBP by 2 percentiles (or $1 \mathrm{mg} / \mathrm{dL}$ increase in plasma uric acid leads to an increase in DBP by 3 percentiles). This is probably not clinically relevant during childhood, nevertheless when maintained during adolescence and adulthood, it can have clinical implications. ${ }^{29,30,34,41,42}$ Prolonged exposure to increased peripheral vascular resistance and high BP leads to embedding of the vessel structure in a remodelled extracellular matrix. ${ }^{43,44}$ Initially, the remodelling of arteries will be adaptive, but it eventually becomes maladaptive and increases stiffness, contributing to cardiovascular complications of hypertension. ${ }^{43}$ Furthermore, an increase in uric acid concentration or $\mathrm{XO}$ activity may not have clinical implication at the time, but it increases the risk of hypertension later in life. ${ }^{30,34,45,46}$ For example, Viazzi et al. showed, among children at high cardiovascular risk, that even a moderately increased serum uric acid concentration at baseline was associated with elevated BP after a mean follow-up of 1.5 years, regardless of appropriate lifestyle changes such as weight loss and diet. ${ }^{34}$ Accordingly, among participants in the Bogalusa Heart Study higher childhood serum uric concentration, even within the normal range, was associated with childhood and adult elevated BP (mean follow-up 11.4-year). ${ }^{30}$

In the current study, BP z-scores were higher than these from the reference population (Table 4). We made use of an oscillometric device to measure BP, whereas the available reference values have been obtained by an auscultatory device. Values obtained by an oscillometric device are considerably higher than these obtained by an auscultatory device. In addition, the average BP measured by the parents was higher than that measured by the trained nurse during the home visits, regardless of time of measurement (morning or evening). This same phenomenon has been observed previously in young adolescents and adults ( $<50$ years), ${ }^{47}$ but in young children it has not been studied extensively. ${ }^{48,49}$ Our results show that these differences already occur in early childhood.

Our study has both strengths and weaknesses that deserve to be commented on. The adjustment for potential confounders, quantification of several purine metabolites, and the repeated BP measurements are major strengths of our study. However, the present study must be interpreted within the context of its potential limitations. First, we would like to emphasize the need for studies examining whether an increased $\mathrm{XO}$ activity causes shifts in several purine metabolite concentrations and their ratios. Second, we 
used a cross-sectional design, so no conclusions concerning causality can be drawn. Third, an oscillometric device was used to measure mean arterial BP, and SBP and DBP were subsequently estimated. Reference data on oscillometric BP measurements would have been more appropriate to create SBP and DBP z-scores. Unfortunately, referential data on oscillometric BP measurements are limited and insufficiently validated. Further studies are required before a complete substitution can be made from using conventional auscultation method to oscillometry. Last, blood samples to determine the purine metabolites were only collected once. As purine concentrations can vary during the day, multiple measures would have been more accurate.

In conclusion, this study showed that plasma uric acid concentration and the ratios uric acid/xanthine and xanthine/hypoxanthine are associated with DBP z-scores in schoolage children. These findings support the role of uric acid as a determinant of BP, already in early childhood, and confirm our hypothesis that an increased $X O$ activity, reflected by increased purine ratios, is associated with BP. These findings highlight the need for further investigation on increased $X O$ activity and $B P$. 


\section{REFERENCES}

1. Wang J, Oin T, Chen J, et al. Hyperuricemia and risk of incident hypertension: a systematic review and meta-analysis of observational studies. PLoS One. 2014;9(12):e114259.

2. Grayson PC, Kim SY, LaValley $M$, et al. Hyperuricemia and incident hypertension: a systematic review and meta-analysis. Arthritis care \& research. 2011;63(1):102-10.

3. Yu MA, Sanchez-Lozada LG, Johnson RJ, et al. Oxidative stress with an activation of the reninangiotensin system in human vascular endothelial cells as a novel mechanism of uric acid-induced endothelial dysfunction. J Hypertens. 2010;28(6):1234-42.

4. Della Corte E, Gozzetti G, Novello F, et al. Properties of the xanthine oxidase from human liver. Biochim Biophys Acta. 1969;191(1):164-6.

5. Guerciolini R, Szumlanski C, Weinshilboum RM. Human liver xanthine oxidase: nature and extent of individual variation. Clin Pharmacol Ther. 1991;50(6):663-72.

6. Stirpe F, Ravaioli M, Battelli MG, et al. Xanthine oxidoreductase activity in human liver disease. Am J Gastroenterol. 2002;97(8): 2079-85.

7. Auscher C, Amory N, van der Kemp P, et al. Xanthine oxidase activity in human intestines. Histochemical and radiochemical study. Adv Exp Med Biol. 1979;122B:197-201.

8. Houston M, Estevez A, Chumley P, et al. Binding of xanthine oxidase to vascular endothelium. Kinetic characterization and oxidative impairment of nitric oxide-dependent signaling. J Biol Chem. 1999;274(8):4985-94.

9. McCord JM, Fridovich I. The reduction of cytochrome c by milk xanthine oxidase. J Biol Chem. 1968;243(21):5753-60.

10. Battelli MG, Bolognesi A, Polito L. Pathophysiology of circulating xanthine oxidoreductase: new emerging roles for a multitasking enzyme. Biochim Biophys Acta. 2014;1842(9):1502-17.

11. So A, Thorens B. Uric acid transport and disease. The Journal of clinical investigation. 2010;120(6):1791-9.

12. Lim SS, Vos T, Flaxman AD, et al. A comparative risk assessment of burden of disease and injury attributable to 67 risk factors and risk factor clusters in 21 regions, 1990-2010: a systematic analysis for the Global Burden of Disease Study 2010. Lancet. 2012;380(9859):2224-60.

13. Tanaka K, Kanazawa I, Yamasaki $\mathrm{H}$, et al. Xanthinuria Type I With a Novel Mutation of
Xanthine Dehydrogenase. $\mathrm{Am} J$ Med Sci. 2015;350(2):155-6.

14. Zhou YN, Zhang XG, Ding R, et al. Using NextGeneration Sequencing to Identify a Mutation in Human MCSU that is Responsible for Type II Xanthinuria. Cell Physiol Biochem. 2015;35(6): 2412-21.

15. Curto R, Voit EO, Sorribas A, et al. Mathematical models of purine metabolism in man. Math Biosci. 1998;151(1):1-49.

16. Feig DI. The role of uric acid in the pathogenesis of hypertension in the young. Journal of clinical hypertension. 2012;14(6):346-52.

17. Kummeling I, Thijs C, Penders J, et al. Etiology of atopy in infancy: the KOALA Birth Cohort Study. Pediatric allergy and immunology : official publication of the European Society of Pediatric Allergy and Immunology. 2005;16(8):679-84.

18. Dutman $A E$, Stafleu $A$, Kruizinga $A$, et al. Validation of an FFO and options for data processing using the doubly labelled water method in children. Public Health Nutr. 2011;14(3):410-7.

19. Scheepers LE, Penders J, Mbakwa CA, et al. The intestinal microbiota composition and weight development in children: the KOALA Birth Cohort Study. International journal of obesity. 2015;39(1): 16-25.

20. Fredriks AM, van Buuren S, Wit JM, et al. Body index measurements in 1996-7 compared with 1980. Arch Dis Child. 2000;82(2):107-12.

21. Ogden $C L$, Flegal KM. Changes in terminology for childhood overweight and obesity. Natl Health Stat Report. 2010(25):1-5.

22. Waterval WA, Scheijen $\mathrm{JL}$, Ortmans-Ploemen MM, et al. Quantitative UPLC-MS/MS analysis of underivatised amino acids in body fluids is a reliable tool for the diagnosis and follow-up of patients with inborn errors of metabolism. Clinica chimica acta; international journal of clinical chemistry. 2009:407(1-2):36-42.

23. Stergiou GS, Yiannes NG, Rarra VC. Validation of the Omron 705 IT oscillometric device for home blood pressure measurement in children and adolescents: the Arsakion School Study. Blood Press Monit. 2006;11(4):229-34.

24. National High Blood Pressure Education Program Working Group on High Blood Pressure in C, Adolescents. The fourth report on the diagnosis, evaluation, and treatment of high blood pressure in children and adolescents. Pediatrics. 2004;114(2 Suppl 4th Report):555-76. 
70 $\mid$ Chapter 3

25. Fang J, Alderman MH. Serum uric acid and cardiovascular mortality the NHANES I epidemiologic follow-up study, 1971-1992. National Health and Nutrition Examination Survey. Jama. 2000;283(18):2404-10.

26. Sun DJY, Li SX, Zhang XT, et al. Uric Acid Is Associated with Metabolic Syndrome in Children and Adults in a Community: The Bogalusa Heart Study. PLoS One. 2014;9(10): e89696.

27. Viazzi F, Antolini L, Giussani M, et al. Serum uric acid and blood pressure in children at cardiovascular risk. Pediatrics. 2013;132(1): e93-9.

28. Jones DP, Richey PA, Alpert BS, et al. Serum Uric Acid and Ambulatory Blood Pressure in Children With Primary Hypertension. Pediatr Res. 2008;64(5):556-61.

29. Feig DI, Johnson RJ. Hyperuricemia in childhood primary hypertension. Hypertension. 2003;42(3):247-52.

30. Alper AB Jr., Chen W, Yau L, et al. Childhood uric acid predicts adult blood pressure: the Bogalusa Heart Study. Hypertension. 2005;45(1):34-8.

31. Gilardini $L$, Parati G, Sartorio A, et al. Sympathoadrenergic and metabolic factors are involved in ambulatory blood pressure rise in childhood obesity. Journal of human hypertension. 2008;22(2):75-82.

32. Noone DG, Marks SD. Hyperuricemia is Associated with Hypertension, Obesity, and Albuminuria in Children with Chronic Kidney Disease. J Pediatr-Us. 2013;162(1):128-32.

33. Pacifico L, Cantisani V, Anania C, et al. Serum uric acid and its association with metabolic syndrome and carotid atherosclerosis in obese children. Eur J Endocrinol. 2009;160(1):45-52.

34. Viazzi F, Rebora P, Giussani M, et al. Increased Serum Uric Acid Levels Blunt the Antihypertensive Efficacy of Lifestyle Modifications in Children at Cardiovascular Risk. Hypertension. 2016;67(5):934-40.

35. Franklin SS, Larson MC, Khan SA, et al. Does the relation of blood pressure to coronary heart disease risk change with aging? The Framingham Heart Study. Circulation. 2001;103(9):1245-9.

36. Li $\mathrm{Y}$, Wei FF, Thijs $\mathrm{L}$, et al. Ambulatory hypertension subtypes and 24-hour systolic and diastolic blood pressure as distinct outcome predictors in 8341 untreated people recruited from 12 populations. Circulation. 2014;130(6):466-74.

37. Li Y, Wei FF, Wang S, et al. Cardiovascular risks associated with diastolic blood pressure and isolated diastolic hypertension. Curr Hypertens Rep. 2014;16(11):489.
38. Corry DB, Eslami P, Yamamoto K, et al. Uric acid stimulates vascular smooth muscle cell proliferation and oxidative stress via the vascular renin-angiotensin system. ] Hypertens. 2008;26(2):269-75.

39. Zhang JX, Zhang YP, Wu QN, et al. Uric acid induces oxidative stress via an activation of the renin-angiotensin system in 3T3-L1 adipocytes. Endocrine. 2015;48(1):135-42.

40. Landmesser U, Spiekermann S, Preuss C, et al. Angiotensin II induces endothelial xanthine oxidase activation: role for endothelial dysfunction in patients with coronary disease. Arteriosclerosis, thrombosis, and vascular biology. 2007;27(4):943-8.

41. Lande MB, Kupferman JC. Pediatric hypertension: the year in review. Clinical pediatrics. 2014;53(4):315-9.

42. Flynn JT. Hypertension in the young: epidemiology, sequelae and therapy. Nephrology, dialysis, transplantation : official publication of the European Dialysis and Transplant Association European Renal Association. 2009;24(2):370-5.

43. Intengan HD, Schiffrin EL. Vascular remodeling in hypertension: roles of apoptosis, inflammation, and fibrosis. Hypertension. 2001;38(3 Pt 2): 581-7.

44. Mulvany MJ. Vascular remodelling of resistance vessels: can we define this? Cardiovasc Res. 1999;41(1):9-13.

45. Sun HL, Pei D, Lue KH, et al. Uric Acid Levels Can Predict Metabolic Syndrome and Hypertension in Adolescents: A 10-Year Longitudinal Study. PLoS One. 2015;10(11): e0143786..

46. Wang JY, Chen YL, Hsu CH, et al. Predictive Value of Serum Uric Acid Levels for the Diagnosis of Metabolic Syndrome in Adolescents. J Pediatr-Us. 2012;161(4):753-6.

47. Ishikawa J, Ishikawa Y, Edmondson D, et al. Age and the difference between awake ambulatory blood pressure and office blood pressure: a meta-analysis. Blood Press Monit. 2011;16(4):159-67.

48. Stergiou GS, Ntineri A, Kollias A, et al. Changing relationship among clinic, home, and ambulatory blood pressure with increasing age. J Am Soc Hypertens. 2015;9(7):544-52.

49. Lurbe E, Thijs L, Redon J, et al. Diurnal blood pressure curve in children and adolescents. J Hypertens. 1996;14(1):41-6.

50. Goncalves JP, Ramos E, Severo M, et al. Serum Uric Acid and Cardiovascular Risk Among Portuguese Adolescents. J Adolescent Health. 2015;56(4):376-81. 
51. Pan $\mathrm{S}, \mathrm{He} \mathrm{CH}, \mathrm{Ma} \mathrm{YT}$, et al. Serum uric acid levels are associated with high blood pressure in Chinese children and adolescents aged 10-15 years. J Hypertens. 2014;32(5):998-1004.

52. Zhonghua. The Society of Pediatrics. The definition of metabolic syndrome and prophylaxis and treatment proposal in Chinese children and adolescents. Zhonghua Er Ke Za Zhi. 2012;50(6): 420-2.

53. Loeffler LF, Navas-Acien A, Brady TM, et al. Uric Acid Level and Elevated Blood Pressure in US Adolescents National Health and Nutrition Examination Survey, 1999-2006. Hypertension. 2012;59(4):811-U145.

54. Hongo $M$, Hidaka $H$, Sakaguchi $S$, et al. Association Between Serum Uric Acid Levels and
Cardiometabolic Risk Factors Among Japanese Junior High School Students. Circulation Journal. 2010;74(8):1570-7.

55. Soergel $M$, Kirschstein $M$, Busch $C$, et al. Oscillometric twenty-four-hour ambulatory blood pressure values in healthy children and adolescents: a multicenter trial including 1141 subjects. J Pediatr. 1997;130(2):178-84.

56. Ford ES, Li CY, Cook S, et al. Serum concentrations of uric acid and the metabolic syndrome among US children and adolescents. Circulation. 2007;115(19):2526-32.

57. Goldstein HS, Manowitz P. Relation between Serum Uric-Acid and Blood-Pressure in Adolescents. Ann Hum Biol. 1993;20(5):423-31. 



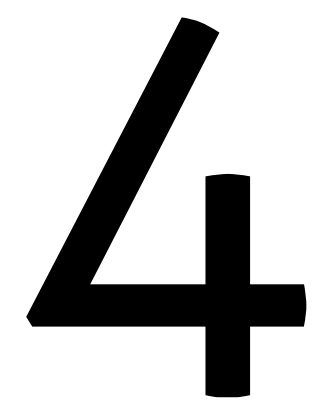

\section{Uric acid and blood pressure: exploring the role of uric acid production in The Maastricht Study}

Lieke Scheepers, Annelies Boonen, Pieter Dagnelie, Miranda Schram, Carla van der Kallen, Ronald Henry, Abraham Kroon, Coen Stehouwer, Ilja Arts 
74 $\mid$ Chapter 4

\section{ABSTRACT}

\section{Objective}

Accumulation of reactive oxygen species by increased uric acid (UA) production has been suggested as a possible underlying mechanism for the association between UA and high blood pressure. We therefore investigated the association between (i) serum UA concentration (ii) 24-h urinary UA excretion, as proxy for UA production, and ambulatory 24-h blood pressure and hypertension.

\section{Methods}

Cross-sectional analyses were conducted among 2555 individuals (52\% men, mean age $60.0 \pm 8.2$ years; $27 \%$ type 2 diabetes [by design]) from The Maastricht Study. Multivariable regression analyses were performed to investigate the association of serum UA and 24-h urinary UA excretion with 24-h pulse pressure (PP), 24-h mean arterial pressure (MAP) and hypertension.

\section{Results}

After adjustment for traditional hypertension risk factors, serum UA concentration (per standard deviation [SD] of $81 \mu \mathrm{mol} / \mathrm{L})$ was associated with higher 24-h MAP ( $\beta 0.63 \mathrm{mmHg}$; 95\% confidence interval $[\mathrm{Cl}]$ 0.27-1.00) and positively associated with hypertension (odds ratio $1.43 ; \mathrm{Cl} 1.27-1.61$ ). Urinary UA excretion (per SD of $140 \mathrm{mg} /$ day $/ 1.73 \mathrm{~m}^{2}$ ) was associated with higher $24-\mathrm{h}$ MAP $(\beta 0.79 \mathrm{mmHg} ; \mathrm{Cl} 0.46-1.12)$ and borderline significantly with hypertension (odds ratio 1.13; $\mathrm{Cl} 1.02-1.25$ ). There was no significant association between serum and 24-h urinary UA excretion with 24-h PP. There was no interaction with sex or age for the aforementioned associations.

\section{Conclusion}

Higher serum and urinary UA concentrations were associated with higher 24-h MAP and hypertension. These results suggest that serum and 24-urinary UA concentrations, the latter as proxy for UA production are, independent of each other, associated with blood pressure and hypertension. 
\begin{tabular}{l|l} 
& \\
Urinary uric acid excretion and blood pressure & 75
\end{tabular}

\section{INTRODUCTION}

Uric acid, the final product of purine catabolism, has been associated with blood pressure and hypertension. Recent meta-analyses showed a significant association between serum uric acid and incident hypertension, independent of traditional risk factors. ${ }^{1,2}$ Several plausible mechanisms have been proposed that causally link uric acid with elevated blood pressure and hypertension. One mechanism includes the activation of the renin-angiotensin-aldosterone system (RAAS) by elevated concentrations of uric acid, leading to increased production of the vasoconstrictor angiotensin $\mathrm{II}^{3}$ Another possible mechanism not directly related to uric acid concentration, but rather to its production, is the generation of reactive oxygen species (ROS) during the production of uric acid. The enzyme xanthine oxidoreductase catalyses the breakdown of hypoxanthine to xanthine and the latter to uric acid. When oxygen is the electron acceptor, superoxide radical anion $\left(\mathrm{O}_{2}{ }^{\bullet-}\right)^{4}$ and hydrogen peroxide $\left(\mathrm{H}_{2} \mathrm{O}_{2}\right)$ are generated as by-products of the oxidation step. These ROS directly reduce the bioavailability of the vasodilator nitric oxide and lead to the formation of peroxynitrite, which can increase endothelial nitric oxide synthase uncoupling resulting in even more ROS formation., ${ }^{5,6}$

Studies published so far have focused mostly on serum uric acid and have ignored the distinction between uric acid concentration and its production. Since the production of uric acid may contribute, independent of uric acid concentration, to the pathogenesis of hypertension, the production should be investigated as well. ${ }^{7}$ Facing the problem that it is not possible to directly measure uric acid production in a large population of individuals, and that uric acid concentration is not an adequate marker for production, proxies for uric acid production need to be investigated. Under normal conditions, the body compensates for increased uric acid production by increasing uric acid excretion, so that serum uric acid remains stable and within the normal range of $200-430 \mu \mathrm{mol} / \mathrm{L}$ for men and 120-340 $\mathrm{mmol} / \mathrm{L}$ for women. ${ }^{8}$ Uric acid is predominantly excreted via the urine; therefore we used 24-h urinary uric acid excretion as a proxy for uric acid production.

In view of the above, we aimed to evaluate whether serum uric acid concentration and/or 24-h urinary uric acid excretion, as proxy for uric acid production are, independent of each other, associated with 24-h ambulatory blood pressure and hypertension. We analysed the pulsatile and steady component of blood pressure captured by 24-h mean arterial pressure (MAP) and 24-h pulse pressure (PP), respectively. 
76 $\mid$ Chapter 4

\section{METHODS}

\section{Study population and design}

We used data from The Maastricht Study, an observational prospective cohort study. The rationale and methodology have been described previously. ${ }^{9}$ In brief, the study focuses on the aetiology, pathophysiology, complications and comorbidities of type 2 diabetes mellitus (T2DM) and is characterized by an extensive phenotyping approach. Eligible for participation were all individuals aged between 40 and 75 years and living in the southern part of The Netherlands. Participants were recruited through mass media campaigns and from municipal registries and the regional Diabetes Patient Registry through mailings. Recruitment was stratified according to known T2DM status, with an oversampling of individuals with T2DM, for reasons of efficiency. The present report includes cross-sectional data from the first 3451 participants, who completed the baseline survey between November 2010 and September 2013. The examinations of each participant were performed within a time window of three months. The study has been approved by the institutional medical ethical committee (NL31329.068.10) and the Minister of Health, Welfare and Sports of The Netherlands (Permit 131088-105234-PG). All participants gave written informed consent.

For the present study we excluded individuals without data on serum uric acid $(n=4)$, creatinine ( $n=6), 24-h$ urine collection ( $n=164)$, ambulatory blood pressure $(n=482)$, level of education ( $n=77)$, smoking status $(n=63)$, alcohol consumption $(n=69)$, body mass index (BMl; $n=3)$, estimated glomerular filtration rate (eGFR) ( $n=33)$, or diabetes duration $(n=105)$. We also excluded individuals with type 1 diabetes mellitus $(n=40)$ or on uric acid lowering therapy (i.e. allopurinol, benzbromarone) $(n=67)$. A total of 2,555 individuals were included in the present analyses (Figure 1).

\section{4-h ambulatory blood pressure measurement}

Ambulatory blood pressure was measured with ambulatory 24-h blood pressure monitoring (WatchBP 03; Microlife AG, Widnau, Switzerland). Cuffs were applied to the participants' non-dominant arm. Measurements were done every 15 minutes during daytime $\left(08: 00_{\text {A.M. }}-11: 00_{\text {P.M. }}\right.$ ) and every 30 minutes during the night (11:00 P.M. 08 :00 A.M. $)$ for a total of 24 hours. Mean 24-h blood pressure measurements were only calculated if there were $>14$ valid measurements at daytime and $>7$ valid measurements at night, according to the recommendation of the British Hypertension Society. ${ }^{10}$ Mean 24-h systolic blood pressure (SBP) and diastolic blood pressure (DBP) were calculated based on hourly averages. ${ }^{11}$ Twenty four-h mean PP was defined as 24-h SBP minus 24-h DBP, and MAP as mean 24-h DBP plus $\left(0.412 \times\right.$ mean $24-h$ PP). ${ }^{12}$ Hypertension was defined as mean 24-h SBP of at least $135 \mathrm{mmHg}, 24-\mathrm{h}$ DBP of at least $85 \mathrm{mmHg}$, or the use of antihypertensive medication. Use of antihypertensive medication was assessed during a medication interview where generic name, dose, and frequency were registered. 


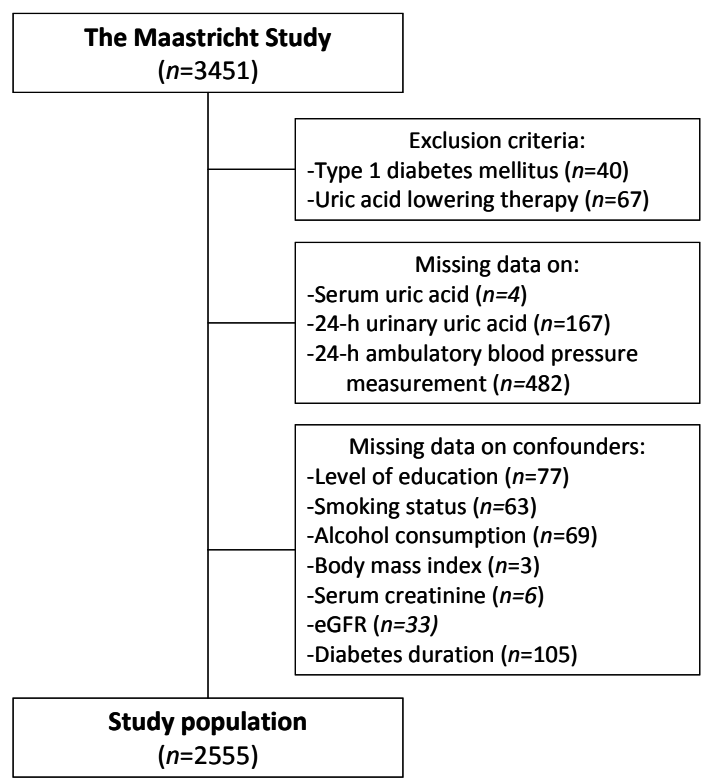

Figure 1. Flow-chart of study participants.

\section{Uric acid determination}

After an overnight fast, venous blood samples were collected to assess serum uric acid and creatinine concentrations with standard (enzymatic and/or colorimetric) methods by and automatic analyser (Beckman Synchron LX20; Beckman Coulter Inc., Brea California, USA or the Roche Cobase601 hs-cTnT assay (Roche) on the Cobas6000 analyser for the last 2585 samples) at Maastricht University Medical Centre (The Netherlands).

To assess urinary uric acid and creatinine excretion, participants were requested to collect a 24-h urine sample. Participants were instructed both orally and in writing on the procedure concerning the 24 -hour urine collection. Only urine collections with a collection time between 20-h and 28-h were considered valid, in case of violation participants were asked to collect urine once more. Urinary uric acid and creatinine concentration were measured with a standard immunoturbidimetric assay by an automatic analyser (Beckman Synchron LX20, Beckman Coulter Inc., Brea, USA) and multiplied by collection volume to obtain the 24-h urinary uric acid excretion. According to the DuBois and DuBois equation, 24-h urinary uric acid and creatinine excretion were adjusted for body surface. ${ }^{13}$

\section{Covariates}

To determine diabetes status, all participants (except those who use insulin) underwent a standardized 2-h $75 \mathrm{~g}$ oral glucose tolerance test after an overnight fast as previously 
described. ${ }^{9}$ Glucose metabolism was defined according to the WHO 2006 criteria into normal glucose metabolism, impaired fasting glucose, impaired glucose tolerance and T2DM. ${ }^{14}$ For this study, we defined having either impaired fasting glucose or impaired glucose tolerance as prediabetes.

Weight and height were measured without shoes and wearing light clothing using a scale and stadiometer to the nearest $0.5 \mathrm{~kg}$ or $0.1 \mathrm{~cm}$ (Seca, Hamburg, Germany). BMI was calculated as body weight $(\mathrm{kg})$ divided by height squared $\left(\mathrm{m}^{2}\right)$. Waist and hip circumference were measured in duplicate midway between the lower rib margin and the iliac crest at the end of expiration and at the widest level over the greater trochanters, respectively (Seca, Hamburg, Germany). Waist-to-hip ratio was calculated as the mean of two waist circumference measurements divided by the mean of two hip circumference measurements. As previously described, ${ }^{9}$ diabetes duration, education level, smoking status, and alcohol consumption were assessed by means of a selfreported questionnaire. Level of education was self-reported and classified into eight categories: 1) no education; 2) primary education; 3) lower vocational education; 4) intermediate general secondary education; 5) intermediate vocational education; 6) higher general secondary education; 7) higher vocational education; and 8) university. For this study, three groups were created for educational level: low (levels 1-3), middle (levels 4-6) and high (levels 7 and 8). Smoking status was based on self-report of smoking cigarettes, cigars and/or pipe tobacco and divided into three categories, i.e. non-smoker, former smoker and current smoker. Alcohol consumption was self-reported as the number of alcohol consumptions per week. One standard alcohol consumption is equivalent to $10 \mathrm{~g}$ (or $13 \mathrm{~mL}$ ) alcohol. This corresponds to one glass of beer of $250 \mathrm{~mL}$ ( $5 \%$ alcohol), one glass of wine of $100 \mathrm{~mL}$ (12\% alcohol), or one glass of spirit of $35 \mathrm{~mL}$ (35\% alcohol). ${ }^{15}$ For analyses, total alcohol consumption was evaluated in grams of alcohol per week. The patients were grouped into three categories, i.e. non-consumers [0 $\mathrm{g}$ of alcohol per week], low consumers $[\leq 70 \mathrm{~g}$ of alcohol per week for females and $\leq 140 \mathrm{~g}$ of alcohol per week for males], and high consumers [ $>70 \mathrm{~g}$ of alcohol per week for females and $>140 \mathrm{~g}$ of alcohol per week for males]." Physical activity was determined from the CHAMPS questionnaire (hours /week). Activities accounted were walking, cycling, gardening, household work, jogging/running, swimming, tennis, team sport, and exercise, regardless whether the activity was on a light or intense. GFR was estimated using the CKD-EPI (Chronic Kidney Disease Epidemiology Collaboration) equation based on both serum creatinine and serum cystatin $C^{16}$ Use of uric acid lowering (allopurinol, febuxostat, probenecid, benzbromarone) and glucose-lowering medication were as well assessed during the medication interview.

\section{Statistical analysis}

The characteristics of the participants are given as mean values \pm standard deviation (SD) for continuous variables and as numbers and proportions for categorical variables. To check if urinary uric acid excretion is an independent marker and does not represent 
serum uric acid concentration we created a scatterplot and calculated the $R^{2}$ between those two variables.

Multiple linear or logistic regression analyses were used to determine the association between the independent variables serum uric acid concentration and 24-h urinary uric acid excretion with the dependent variables ambulatory 24-h PP, 24-h MAP and the odds ratio (OR) of prevalent hypertension. In the first model, the results of the crude analyses were presented. In the second model, crude results were adjusted for sex, age, glucose metabolism status, smoking status, alcohol consumption, eGFR, level of education, use of diabetes medication (no, oral medication, insulin with or without oral medication), RAASinhibitors and other antihypertensive medication (including beta-blockers) that have no known uricosuric properties, losartan (known as a RAAS inhibitor with a uricosuric effect), ${ }^{17,18}$ and antihypertensive medication and lipid-lowering medication that may have a uricosuric effect (i.e. secondary uricosurics, amlodipine, ${ }^{19}$ atorvastatin, ${ }^{20}$ rosuvastatin). ${ }^{20}$ In third model, the analyses with serum uric acid were additionally adjusted for 24-h urinary uric acid excretion; and the associations with 24-h urinary uric acid excretion were additionally adjusted for serum uric acid. To explore of serum and urinary uric acid concentrations are, independent of each other, associated with blood pressure and hypertension. The logistic regression models were adjusted for the same covariates, except for the use of antihypertensive medication, which is part of the definition of the outcome. As it has been suggested that uric acid has a more pronounced effect in younger and female individuals, we investigated whether the association between serum uric acid concentration and urine uric acid excretion with ambulatory blood pressure and hypertension differed with gender and age. ${ }^{21-24}$ Since an elevated uric acid may be a consequence of a high BMI or waist-to-hip ratio, adjustment for BMI or waist-to-hip ratio in the analyses may lead to overadjustment and these variables were therefore not included in the main analyses. In a sensitivity analysis we additionally adjusted for BMI or waist-to-hip ratio. ${ }^{25}$ Due to the large number of missing values on physical activity $(n=278)$ this variable was not included as potential confounder in the main analyses. A sensitivity analysis was performed to control for this variable in the subset of participants with complete data. The null hypothesis was rejected for a two-sided $P$-value was less than 0.05, except for the interaction analyses, where a $P$-value less than 0.10 was used. Analyses were conducted using SPSS version 23 for windows (SPSS, Inc.).

\section{RESULTS}

The overall study population consisted of 2555 individuals with an average age of 59.8 (SD 8.1) years, $51 \%$ of whom were men. Due to oversampling of individuals with diabetes $24 \%(n=606)$ had T2DM. Table 1 shows the general characteristics of the study population and the individuals excluded from the analyses. 
80 $\mid$ Chapter 4

Table 1. Characteristics of The Maastricht Study population and the individuals excluded from the analyses because of missing values

\begin{tabular}{|c|c|c|c|}
\hline & $\begin{array}{l}\text { Study population } \\
\quad(n=2,555)\end{array}$ & Missing & $\begin{array}{c}\text { Excluded because of } \\
\text { missing values } \\
(n=896)\end{array}$ \\
\hline Serum uric acid ( $\mu \mathrm{mol} / \mathrm{L})$ & $329.3 \pm 81.3$ & 6 & $331.8 \pm 85.00$ \\
\hline Serum creatinine (umol/L) & $77.2 \pm 16.1$ & 4 & $77.4 \pm 17.7$ \\
\hline Urine uric acid excretion (mg/day/1.73 $\mathrm{m}^{2}$ ) & $518.1 \pm 139.92$ & 127 & $507.5 \pm 181.6$ \\
\hline Urine creatinine excretion ( $\mathrm{mmol} /$ day $/ 1.73 \mathrm{~m}^{2}$ ) & $12.0 \pm 2.8$ & 78 & $11.9 \pm 4.1$ \\
\hline Fractional uric acid excretion ( $\mathrm{FE}_{\mathrm{UA}}$ ) & $9.3 \% \pm 5.3$ & 173 & $9.5 \% \pm 10.4$ \\
\hline Age (years) & $59.8 \pm 8.1$ & 0 & $59.6 \pm 8.6$ \\
\hline Male sex (n) & $51.0 \%(1303)$ & 0 & $52.7 \%(472)$ \\
\hline $\operatorname{BMI}\left(\mathrm{kg} / \mathrm{m}^{2}\right)$ & $26.8 \pm 4.3$ & 5 & $27.9 \pm 5.1$ \\
\hline Waist-to-hip ratio & $0.94 \pm 0.09$ & 4 & $0.96(0.10)$ \\
\hline Smoking status,(n) & & 63 & \\
\hline Never & $35.9 \%(916)$ & & $28.3 \%(254)$ \\
\hline Past & $52.2 \%(1333)$ & & $46.4 \%(416)$ \\
\hline Current & $12.0 \%(306)$ & & $18.2 \%(163)$ \\
\hline Alcohol consumption, ( $n$ ) & & 69 & \\
\hline No & $16.9 \%(432)$ & & $22.0 \%(197)$ \\
\hline Low & $56.6 \%(1446)$ & & $47.9 \%(429)$ \\
\hline High & $26.5 \%(677)$ & & $22.4 \%(201)$ \\
\hline Educational level, (n) & & 77 & \\
\hline Low & $31.4 \%(801)$ & & $37.1 \%(332)$ \\
\hline Medium & $28.6 \%(731)$ & & $24.8 \%(222)$ \\
\hline High & $40.0 \%(1,023)$ & & $29.6 \%(265)$ \\
\hline Physical activity (hours/week)* & $14.5 \pm 8.2$ & 179 & $13.0 \pm 7.8$ \\
\hline eGFR $\left(\mathrm{ml} / \mathrm{min}\right.$ per $\left.1.73 \mathrm{~m}^{2}\right)$ & $88.25 \pm 14.50$ & 55 & $87.7 \pm 16.5$ \\
\hline Glucose metabolism status, (n) & & 0 & \\
\hline Normal glucose metabolism & $60.6 \%(1549)$ & & $41.9 \%(375)$ \\
\hline Impaired fasting glucose & $4.3 \%(111)$ & & $3.6 \%(32)$ \\
\hline Impaired glucose tolerance & $11.3 \%(289)$ & & $8.8 \%(79)$ \\
\hline Type 2 diabetes mellitus & $23.7 \%(606)$ & & $41.2 \%(369)$ \\
\hline Other type of diabetes & $\mathrm{n} / \mathrm{a}$ & & $4.5 \%(41)$ \\
\hline Diabetes treatment among individuals with T2DM, (n) & & 4 & \\
\hline No medication & $25.1 \%(152)$ & & $15.5 \%(57)$ \\
\hline Oral medication & $55.0 \%(333)$ & & $58.7 \%(216)$ \\
\hline Insulin with or without oral medication & $20.0 \%(121)$ & & $25.8 \%(95)$ \\
\hline Diabetes duration (years), median (range) & $8.4 \pm 7.2$ & 178 & $7.4 \pm 6.9$ \\
\hline Hypertension, (n) & $43.2 \%(1103)$ & 406 & $59.8 \%(293)$ \\
\hline Use of antihypertensive medication, (n) & $37.1 \%(947)$ & 4 & $44.6 \%(353)$ \\
\hline RAAS-inihibitors & $24.7 \%(631)$ & & $34.3 \%(307)$ \\
\hline Other antihypertensives, no uricosuric effect & $20.6 \%(527)$ & & $30.7 \%(275)$ \\
\hline Losartan & $2.9(75)$ & & $3.8 \%(34)$ \\
\hline Secondary uricosuric & $13.5 \%(346)$ & & $20.4 \%(182)$ \\
\hline Mean arterial pressure 24-h (mmHg) & $88.5 \pm 7.9$ & 404 & $89.8 \pm 8.5$ \\
\hline Pulse pressure $24-\mathrm{h}(\mathrm{mmHg})$ & $45.2 \pm 8.5$ & 404 & $47.5 \pm 9.8$ \\
\hline
\end{tabular}

Data are presented as mean and standard deviation (SD) unless otherwise indicated.

*Missing data for 278 individuals on variable physical activity.

On average the serum uric acid concentration was $329 \mu \mathrm{mol} / \mathrm{L}$ (SD 81) and 24-h urinary uric excretion was $518 \mathrm{mg} /$ day $/ 1.73 \mathrm{~m}^{2}$ (SD 140). In total 1,103 individuals (43\%) had hypertension, of these 947 (86\%) were on antihypertensive medication. In the 
remaining 156 patients (14\%), the diagnosis of hypertension relied on the threshold being exceeded for SBP ( $n=49 ; 31 \%), \operatorname{DBP}(n=45 ; 29 \%)$, or both $(n=62 ; 40 \%)$. Individuals excluded from analyses had a lower level of education, slightly higher BMI, and were more often T2DM or hypertensive patients.

The variance in serum uric acid, while significant ( $P$-value $<0.001)$, explains only $0.9 \%$ of the variance in urinary uric acid excretion (Figure 2 ).

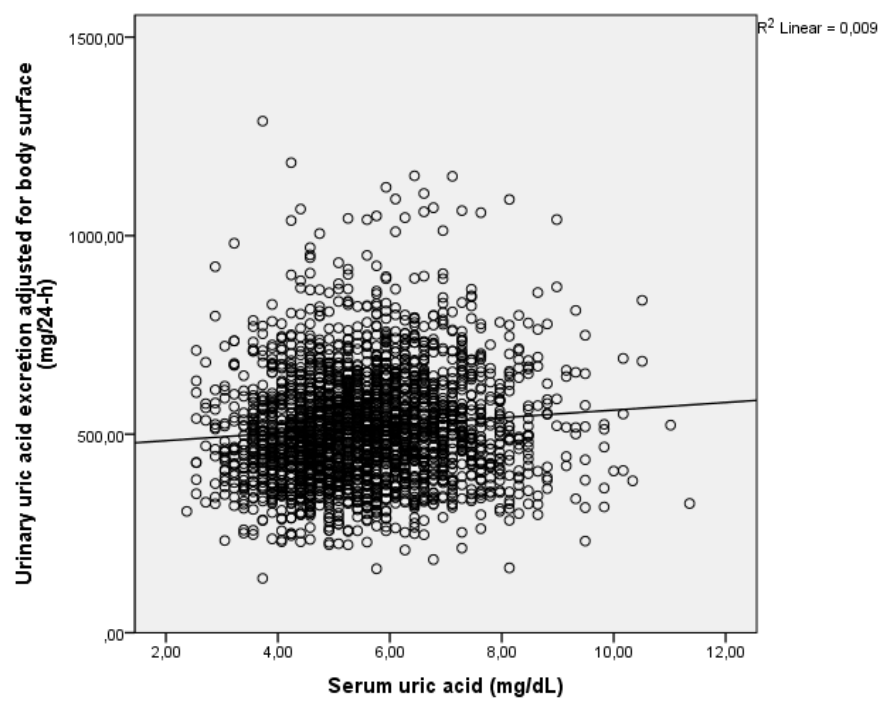

Figure 2. Uric acid excretion in 24-h urine $\left(\mathrm{mg} / 1.73 \mathrm{~m}^{2}\right)$ as a function of serum uric acid $(\mathrm{mg} / \mathrm{dL})$ in 2555 participants from The Maastricht Study.

\section{Serum uric acid and 24-h blood pressure}

Crude linear regression analysis showed that a 1 SD $(81 \mu \mathrm{mol} / \mathrm{L})$ higher serum uric acid concentration was associated with $1.73 \mathrm{mmHg}(95 \%$ confidence interval $[\mathrm{Cl}] 1.44$ to $2.02 \mathrm{mmHg}$ ) higher 24-h MAP and greater odds for hypertension (OR 1.89; Cl 1.73 to 2.07) (Table 2, model 1). Further adjustment for sex, age, and glucose metabolism status, smoking status, alcohol consumption, education, eGFR, and use of antihypertensive or diabetes medication, did not materially change the result (model 2). Furthermore, results remained significant with additional adjustment for 24-h urinary uric acid excretion (model 3). The association with 24-h PP was significant in the crude analysis (Table 2, model 1), but lost significance after adjustment for potential confounders (models 2 and 3). 
$82 \mid$ Chapter 4

Table 2. Associations between serum uric acid and urinary uric acid excretion with ambulatory mean arterial pressure, pulse pressure and hypertension

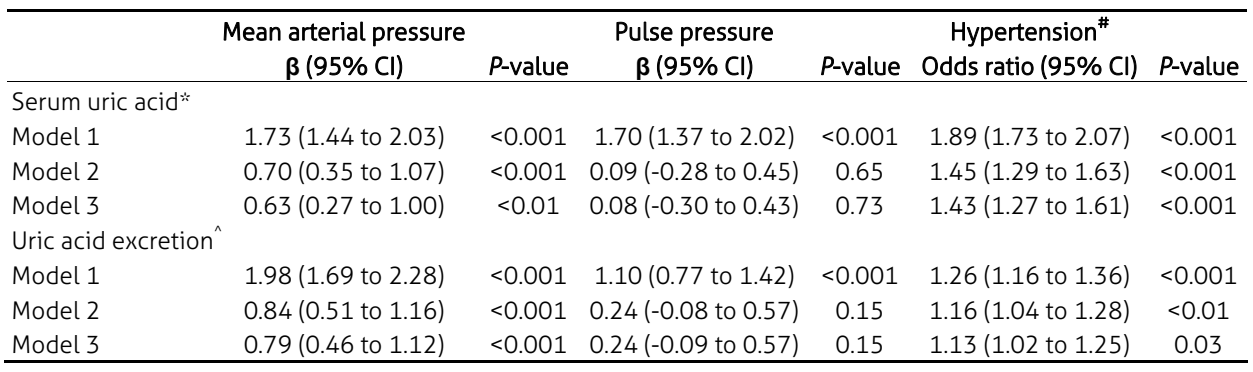

* Serum uric acid expressed per standard deviation ( $81 \mu \mathrm{mol} / \mathrm{L})$. ^ Uric acid excretion in 24-h urine expressed per standard deviation $\left(140 \mathrm{mg} /\right.$ day $/ 1.73 \mathrm{~m}^{2}$ ). " Hypertension is defined as a $24-\mathrm{h}$ SBP of $>135$, or DBP>85 or the use of anti-hypertensive medication. In the adjusted analyses, no adjustment for antihypertensive medication. Model 1: Crude. Model 2 adjusted for age, sex, glucose metabolism status (normal, impaired, T2DM), smoking status (never, current, former), alcohol consumption (no, low, high), education (low, middle, high), eGFR, use of diabetes medication (no, insulin with or without oral medication) and antihypertensive medication (no, RAAS inhibitors, diuretics or other beta blockers and calcium), and duration of diabetes. Model 3, analyses with serum uric acid were additionally adjusted for 24-h urinary uric acid excretion; and the associations with 24-h urinary uric acid excretion were additionally adjusted for serum uric acid.

\section{4-h urine uric acid excretion and 24-h blood pressure}

Crude linear regression analysis showed that a 1 SD higher 24-h urinary uric acid excretion (140 mg/day/1.73 $\left.\mathrm{m}^{2}\right)$ was associated with higher 24-h MAP ( $\beta$ 1.98; Cl 1.69 to $2.28 \mathrm{mmHg}$ ) and greater odds for hypertension (OR 1.26; $\mathrm{Cl} 1.16$ to 1.36) (Table 2, model 1). The associations of serum uric acid with 24-h MAP and hypertension did not materially change after further adjustment for potential confounders (model 2).

Further adjustment for serum uric acid concentration did not materially changes the results (model 3 ). The association between urinary uric acid excretion and 24-h PP was significant in the crude analysis ( $\beta$ 1.10; $\mathrm{Cl} 0.77$ to $1.42 \mathrm{mmHg}$ ), but lost significance after adjustment for potential confounders (Table 2, models 2 and 3; $P$-value $<0.15$ ).

\section{Effect modification by age or sex}

To determine whether the associations between serum uric acid and urine uric acid excretion with ambulatory PP, MAP and hypertension were different across sex and age strata, an interaction term was added to the fully adjusted regression models. No significant interaction with sex or age was identified in any of the investigated associations ( $P$-value $>0.05)$.

\section{Sensitivity analyses}

Including BMI or waist-to-hip ratio as a covariate to model 3 did not materially alter the associations between serum uric acid and blood pressure or hypertension. However, the 
\begin{tabular}{l|l} 
& \\
Urinary uric acid excretion and blood pressure & 83
\end{tabular}

association between urinary uric acid excretion and hypertension was attenuated after including both BMI (OR 0.99; $P=0.80$ ) and waist-to-hip ratio (OR 1.04; $P=0.45$ ). Additional adjustment for physical activity did not materially alter the associations of serum uric acid and urinary uric acid excretion with ambulatory PP, MAP and hypertension (data not shown).

\section{DISCUSSION}

This study represents a comprehensive analysis of the association of uric acid with ambulatory blood pressure and hypertension in middle-aged individuals. Serum uric acid concentration and 24-h urinary uric acid excretion, as proxy for uric acid production, were independent of each other associated with ambulatory MAP and hypertension. To the best of our knowledge, this study is the first to show an independent association of 24-h urinary uric acid excretion, as proxy for uric acid production, with ambulatory blood pressure and hypertension.

Our results are in line with prior research showing that serum uric acid was associated with hypertension, independent of traditional hypertension risk factors. ${ }^{2} \mathrm{~A}$ meta-analysis performed by Wang et al. in 2014, showed that a $1 \mathrm{mg} / \mathrm{dL}$ increase in uric acid was associated with an increased risk of incident hypertension (adj. relative risk $1.15 ; \mathrm{Cl} 1.06$ to 1.26$)$. In the current cross-sectional study a similar odds ratio of 1.20 ( $\mathrm{Cl} 1.10$ to 1.31 ) for each $1 \mathrm{mg} / \mathrm{dL}$ higher serum uric acid concentration was found. In the present study, we found no age or sex related difference in the association between serum uric acid and any of the outcomes. This is in contrast with the meta-analysis performed by Grayson at al. which showed that the risk of hypertension among individuals with hyperuricemia was significantly larger in younger individuals and women. ${ }^{1}$ This contrasting finding may be attributed to the inclusion criterium of an age of 40 years and older, resulting in a relative high age of our study population (mean age of 60.0 years).

There are no earlier studies examining the association between urinary uric acid excretion, as proxy for uric acid production, and blood pressure. We showed that urinary uric acid excretion was significantly associated with ambulatory MAP and hypertension, after adjustment for potential confounders including serum uric acid. These findings confirm our hypothesis that increased uric acid production is associated with blood pressure and hypertension. Uric acid is produced during the metabolism of endogenous (DNA, RNA and ATP) and exogenous (dietary) purines. Previous studies showed that an increase in uric acid production, either by endogenous or exogenous supply, increases urinary uric acid excretion. ${ }^{8}$ Approximately $70 \%$ of the produced uric acid is eliminated by the kidney and $30 \%$ by the intestine (extra-renal pathway), whether the relative contribution of renal and extra-renal excretion is comparable in case of increased uric acid production needs investigation. ${ }^{26}$ In case of decreased extra-renal excretion, serum uric acid and urinary uric acid excretion will increase. Among white individuals approximately 11\% (HapMap, CHB, and JPT) has the Q141K mutation in the urate 
$84 \mid$ Chapter 4

transport $A B C G 2$, which leads to a decrease in extra-renal uric acid excretion, causing an increased serum and urinary uric acid concentrations and eventually gout. ${ }^{27-29}$ Theoretically, the "overproducers" in the current concept, those with increased urinary uric acid excretion, may include next to the genuine "uric acid overproducers" also "underexcretors of uric acid via the intestine (extra-renal elimination)". ${ }^{27}$ Unfortunately it is not feasible to determine uric acid excretion via the intestine since it is degraded by uricase activity of the intestinal microbiota, leading to an almost complete lack of uric acid in the faeces. ${ }^{30}$ However, by excluding patients on uric acid lowering therapy, we have attempted to exclude those with gout and therefore possibly with decreased renal or extra-renal excretion.

In addition, oxidation of hypoxanthine to xanthine does not have to be equivalent to the oxidation of xanthine to uric acid. Several feedback mechanisms can control the supply of xanthine and thereby the corresponding uric acid production. ${ }^{31}$ For example, an increased urinary excretion of xanthine ${ }^{32}$ or degradation of xanthine to xanthosine monophosphate by certain hypoxanthine-guanine phosphoribosyltransferase ${ }^{33}$ decreases the xanthine concentration. If this is the case, increased oxidation of hypoxanthine to xanthine and the associated harmful accumulation of ROS will not lead to an increased uric acid production. In the future a systematic approach integrating the supply of hypoxanthine and xanthine and the involved pathways might be a more accurate way to determine uric acid production.

After adjustment for potential confounders (including serum uric acid) the association between urinary uric acid excretion and hypertension remained significant (Table 2 , model 3). Further adjustment for BMI $(P=0.80)$ or waist-to-hip ratio $(P=0.45)$ attenuated the association. This might be explained by the fact that adiposity increases xanthine oxidoreductase activity in mice, thus leading to overproduction of uric acid. ${ }^{25}$ Adjustment for BMI or waist-to-hip ratio (measure for abdominal visceral fat), might therefore lead to overadjustment. Further research is required to examine the influence of adiposity and visceral fat on uric acid production and its association with hypertension. The associations of serum uric acid with blood pressure and hypertension were not attenuated after including BMI or waist-to-hip ratio to the fully adjusted model. This is in line with the idea that serum uric acid concentration does not reflect uric acid production, since an increased uric acid production is compensated by increased excretion to maintain serum uric acid within the normal range.

In the present study serum uric acid and urinary uric acid excretion were associated with MAP but not with PP. MAP is the steady component of blood pressure reflecting vascular resistance, which can be increased by inhibiting the vasodilator nitric oxide. As previously described, several plausible mechanisms have been shown to link uric acid with a decrease in the bioavailability of nitric oxide. PP, the pulsatile component of blood pressure, increases as a consequence of prolonged exposure to increased vascular resistance and elevated blood pressure. This results in remodelling of the vascular extracellular matrix, ${ }^{34,35}$ stiffening of the arteries and ultimately a loss in vascular compliance. Thus far, no prior studies investigated the association between uric acid and 
\begin{tabular}{l|l} 
& \\
Urinary uric acid excretion and blood pressure & 85
\end{tabular}

pulse pressure in depth. ${ }^{36-41}$ Previous studies investigated the association between uric acid and arterial stiffness. In line with the current results, a study conducted in a subset of The Maastricht Study population ( $n=614$ ), showed no association of serum uric acid with stiffness of the aorta, or the carotid or femoral artery. ${ }^{42}$ Nonetheless, conclusive evidence cannot be provided since the literature shows disparate results. ${ }^{43-47} \mathrm{We}$ therefore emphasize the need for further studies addressing the role of uric acid on pulse pressure and arterial stiffness. In particular longitudinal studies including middleaged individuals, since uric acid may lead to early changes in arterial stiffness and subsequently to elevated PP, but may have less influence once vascular damage is permanent. $^{48}$

The use of 24-h ambulatory blood pressure measurements and the adjustment for a large number of carefully measured potential confounders are major strengths of our study. Our study also has some limitations which should be considered. We would like to emphasize the need for further research validating urinary uric acid excretion as a proxy for uric acid production. Furthermore, we proposed that accumulation of reactive oxygen species is a potential underlying mechanism linking increased uric acid production with elevated blood pressure, but from the present study no conclusion can be drawn concerning the actual mechanism. Due to the cross-sectional nature of this study, causal relationships could not be determined. In addition, due to missing data, we had to exclude almost 900 participants. Although we assumed the random nature of these missing because most values were missing due to logistic factors (e.g. the temporary unavailability of ambulatory blood pressure monitors), the excluded individuals had a higher prevalence of (pre)diabetes and hypertension (Table 1). Furthermore, serum uric acid and 24-h urinary uric acid excretion was only determined once. Since uric acid concentrations can vary between days, multiple measures would have been more accurate. Finally, our study population consisted of relatively more individuals with T2DM between 40-75 years of age; therefore, the results might not be representative for the general population.

\section{CONCLUSION}

We found evidence for associations between serum uric acid and urinary uric acid excretion with ambulatory MAP and hypertension. By studying urinary uric acid excretion, we aimed to investigate whether an increased uric acid production was, independent of serum uric acid, associated with blood pressure. Finding significant associations supports our hypothesis, highlighting the need for further investigation on increased uric acid production and its effect on urinary uric acid excretion and blood pressure. 
$86 \mid$ Chapter 4

\section{REFERENCES}

1. Grayson PC, Kim SY, LaValley $M$, et al. Hyperuricemia and incident hypertension: a systematic review and meta-analysis. Arthritis Care Res (Hoboken). 2011; 63(1): 102-110.

2. Wang J, Oin T, Chen J, et al. Hyperuricemia and risk of incident hypertension: a systematic review and meta-analysis of observational studies. PLoS One. 2014; 9(12):e114259.

3. Yu MA, Sanchez-Lozada LG, Johnson RJ, et al. Oxidative stress with an activation of the reninangiotensin system in human vascular endothelial cells as a novel mechanism of uric acid-induced endothelial dysfunction. J Hypertens. 2010; 28(6):1234-1242.

4. McCord JM, Fridovich I. The reduction of cytochrome c by milk xanthine oxidase. J Biol Chem. 1968; 243(21):5753-5760.

5. Corry DB, Eslami P, Yamamoto K, et al. Uric acid stimulates vascular smooth muscle cell proliferation and oxidative stress via the vascular renin-angiotensin system. J Hypertens. 2008; 26(2):269-275.

6. Zhang JX, Zhang YP, Wu QN, et al. Uric acid induces oxidative stress via an activation of the renin-angiotensin system in 3T3-L1 adipocytes. Endocrine. 2015; 48(1):135-142.

7. Scheepers LE, Wei FF, Stolarz-Skrzypek K, et al. Xanthine oxidase gene variants and their association with blood pressure and incident hypertension: a population study. J Hypertens. 2016; 34(11):2147-2154.

8. Puig JG, Torres RJ, de Miguel E, et al. Uric acid excretion in healthy subjects: a nomogram to assess the mechanisms underlying purine metabolic disorders. Metabolism. 2012; 61(4):512-518

9. Schram MT, Sep SJ, van der Kallen C], et al. The Maastricht Study: an extensive phenotyping study on determinants of type 2 diabetes, its complications and its comorbidities. European journal of epidemiology. 2014; 29(6):439-451.

10. O'Brien E, Coats $A$, Owens $P$, et al. Use and interpretation of ambulatory blood pressure monitoring: recommendations of the British Hypertension Society. British Medical Journal. 2000; 320(7242):1128-1134.

11. Mancia G, Fagard R, Narkiewicz K, et al. 2013 ESH/ESC Guidelines for themanagement of arterial hypertension The Task Force for the management ofarterial hypertension of the European Society ofHypertension (ESH) and of the European Society of Cardiology (ESC). J Hypertens. 2013; 31(7):1281-1357.

12. Meaney E, Alva F, Moguel R, et al. Formula and nomogram for the sphygmomanometric calculation of the mean arterial pressure. Heart. 2000; 84(1):64.

13. Dubois D, Dubois EF. Nutrition Metabolism Classic - a Formula to Estimate the Approximate Surface-Area If Height and Weight Be Known (Reprinted from Archives Internal Medicine, Vol 17, Pg 863, 1916). Nutrition. 1989; 5(5):303311.

14. Definition and Diagnosis of Diabetes Mellitus and Intermediate Hyperglycemia: World Health Organization;2006.

15. Richtlijnen goede voeding 2015. 2015, Health Council of The Netherlands (Gezondheidsraad).

16. Inker LA, Schmid CH, Tighiouart $H$, et al. Estimating glomerular filtration rate from serum creatinine and cystatin C. N Engl J Med. 2012; 367(1):20-29.

17. Takahashi S, Moriwaki Y, Yamamoto T, et al. Effects of combination treatment using antihyperuricaemic agents with fenofibrate and/or losartan on uric acid metabolism. Ann Rheum Dis. 2003; 62(6):572-575.

18. Hamada T, Ichica K, Hosoyamada $M$, et al. Uricosuric action of losartan via the inhibition of urate transporter 1 (URAT1) in hypertensive patients. Am J Hypertens. 2008; 21(10):11571162.

19. Chanard J, Toupance O, Lavaud S, et al. Amlodipine reduces cyclosporin-induced hyperuricaemia in hypertensive renal transplant recipients. Nephrol Dial Transpl. 2003; 18(10):2147-2153.

20. Ogata N, Fujimori S, Oka Y, et al. Effects of Three Strong Statins (Atorvastatin, Pitavastatin, and Rosuvastatin) on Serum Uric Acid Levels in Dyslipidemic Patients. Nucleos Nucleot Nucl. 2010; 29(4-6):321-324.

21. Brand FN, McGee DL, Kannel WB, et al. Hyperuricemia as a risk factor of coronary heart disease: The Framingham Study. Am J Epidemiol. 1985; 121(1):11-18.

22. Kannel WB. Metabolic risk factors for coronary heart disease in women: perspective from the Framingham Study. Am Heart J. 1987; 114(2): 413-419.

23. Watanabe $S$, Kang DH, Feng $L$, et al. Uric acid, hominoid evolution, and the pathogenesis of 
\begin{tabular}{l|l} 
& \\
Urinary uric acid excretion and blood pressure & 87
\end{tabular}

salt-sensitivity. Hypertension. 2002; 40(3):355360.

24. Samimi A, Ramesh S, Turin TC, et al. Serum uric acid level, blood pressure, and vascular angiotensin $\|$ responsiveness in healthy men and women. Physiological reports. 2014; 2(12).

25. Tsushima $Y$, Nishizawa $H$, Tochino $Y$, et al. Uric acid secretion from adipose tissue and its increase in obesity. J Biol Chem. 2013; 288(38):27138-27149.

26. Hosomi A, Nakanishi T, Fujita T, et al. Extra-renal elimination of uric acid via intestinal efflux transporter BCRP/ABCG2. PLoS One. 2012; 7(2): e30456.

27. Matsuo H, Takada T, Nakayama A, et al. ABCG2 dysfunction increases the risk of renal overload hyperuricemia. Nucleosides Nucleotides Nucleic Acids. 2014; 33(4-6):266-274.

28. Uhlen $M$, Fagerberg $L$, Hallstrom $B M$, et al. Proteomics. Tissue-based map of the human proteome. Science. 2015; 347(6220):1260419.

29. Takada $T$, Ichida $K$, Matsuo $H$, et al. ABCG2 dysfunction increases serum uric acid by decreased intestinal urate excretion. Nucleosides Nucleotides Nucleic Acids. 2014; 33(4-6):275281.

30. Sorensen LB. Degradation of uric acid in man. Metabolism. 1959; 8:687-703.

31. Lane AN, Fan TW. Regulation of mammalian nucleotide metabolism and biosynthesis. Nucleic Acids Res. 2015; 43(4):2466-2485.

32. Puig JG, Mateos FA, Jimenez ML, et al. Renal excretion of hypoxanthine and xanthine in primary gout. Am J Med. 1988; 85(4):533-537.

33. Curto R, Voit EO, Sorribas A, et al. Mathematical models of purine metabolism in man. Math Biosci. 1998; 151(1):1-49.

34. Intengan HD, Schiffrin EL. Vascular remodeling in hypertension: roles of apoptosis, inflammation, and fibrosis. Hypertension. 2001; 38(3 Pt 2):581-587.

35. Mulvany MJ. Vascular remodelling of resistance vessels: can we define this? Cardiovasc Res. 1999; 41(1):9-13.

36. Casiglia E, Tikhonoff V, Mazza A, et al. Pulse pressure and coronary mortality in elderly men and women from general population. J Hum Hypertens. 2002; 16(9):611-620.

37. Inoue $T$, Matsuoka $M$, Nagahama $K$, et al. Cardiovascular risk factors associated with pulse pressure in a screened cohort in Okinawa, Japan. Hypertens Res. 2003; 26(2):153-158.
38. Tsioufis C, Kyvelou S, Dimitriadis K, et al. The diverse associations of uric acid with low-grade inflammation, adiponectin and arterial stiffness in never-treated hypertensives. J Hum Hypertens. 2011; 25(9):554-559.

39. Jin $Y L, Z h u T, X u L$, et al. Uric acid levels, even in the normal range, are associated with increased cardiovascular risk: the Guangzhou Biobank Cohort Study. Int J Cardiol. 2013; 168(3):22382241.

40. Mazza A, Pessina AC, Gianluca P, et al. Pulse pressure: an independent predictor of coronary and stroke mortality in elderly females from the general population. Blood Press. 2001; 10(4):205-211.

41. Wiik BP, Larstorp ACK, Hoieggen A, et al. Serum Uric Acid is Associated With New-Onset Diabetes in Hypertensive Patients With Left Ventricular Hypertrophy: The LIFE Study. Am J Hypertens. 2010; 23(8):845-851.

42. Wijnands JM, Boonen A, van Sloten TT, et al. Association between serum uric acid, aortic, carotid and femoral stiffness among adults aged 40-75 years without and with type 2 diabetes mellitus: The Maastricht Study. J Hypertens. 2015; 33(8):1642-1650.

43. Chen X, Li Y, Sheng CS, et al. Association of serum uric acid with aortic stiffness and pressure in a Chinese workplace setting. Am J Hypertens. 2010; 23(4): 387-392.

44. Ishizaka N, Ishizaka $Y$, Toda E, et al. Higher serum uric acid is associated with increased arterial stiffness in Japanese individuals. Atherosclerosis. 2007; 192(1):131-137.

45. Kuo CF, Yu KH, Luo SF, et al. Role of uric acid in the link between arterial stiffness and cardiac hypertrophy: a cross-sectional study. Rheumatology (Oxford). 2010; 49(6):1189-1196.

46. Lim JH, Kim YK, Kim YS, et al. Relationship between serum uric Acid levels, metabolic syndrome, and arterial stiffness in korean. Korean Circ J. 2010; 40(7): 314-320.

47. Hsu PF, Chuang SY, Cheng HM, et al. Associations of serum uric acid levels with arterial wave reflections and central systolic blood pressure. Int J Cardiol. 2013; 168(3):20572063.

48. Feig DI. The role of uric acid in the pathogenesis of hypertension in the young. J Clin Hypertens (Greenwich). 2012; 14(6):346-352. 



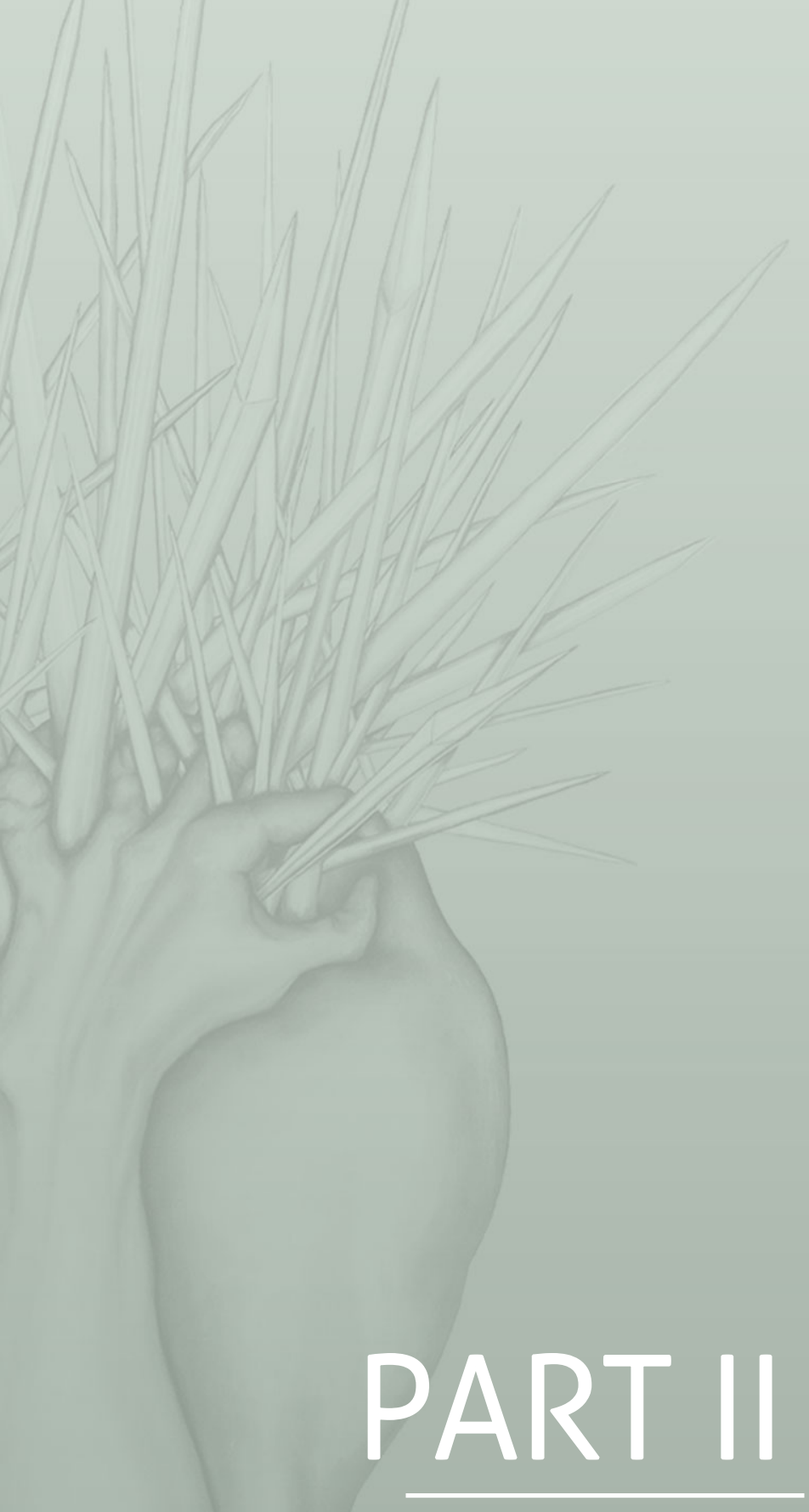

Gout management by the patient and general practitioner 



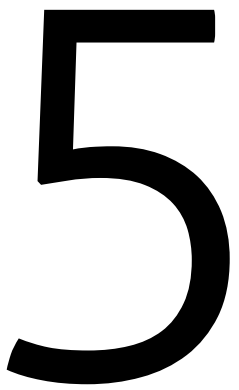

Medication adherence among gout patients initiated allopurinol: a retrospective cohort study in the Clinical Practice Research Datalink 


\section{ABSTRACT}

\section{Objective}

To assess medication adherence, including non-persistence, non-adherence and restarting therapy, among newly diagnosed gout patients initiated allopurinol and to identify factors associated with poor medication adherence.

\section{Methods}

A retrospective cohort study was conducted within the UK Clinical Practice Research Datalink (1987-2014) among incident gout patients, aged $\geq 40$ years and initiating allopurinol $(n=48,280)$. Medication adherence for allopurinol treatment was described by non-persistence (occurrence of a first gap of $\geq 90$ days), non-adherence (proportion of days covered $[P D C]<80 \%$ over observation period), restart of therapy after the first gap and subsequent medication adherence. Kaplan Meier survival and multivariable Coxand logistic regression were used to estimate non-persistence and median time until discontinuation, and factors associated with non-persistence or non-adherence, respectively.

\section{Results}

Non-persistence increased from 38.5\% (95\% confidence interval [Cl] 38.1-38.9) to $56.9 \%(\mathrm{Cl} 56.4-57.4)$ after 1 and 5 years of initiation, respectively. Median survival time until a first 90-day gap was 225 days (Cl 220-231). Median PDC was 0.67 (IOR: 0.65) and $62 \%$ of the patients were considered non-adherent. After the occurrence of a first gap, 43.3\% (Cl 42.7-43.9) restated therapy within 1 year, yet only $52.3 \%(\mathrm{Cl} 51.4-53.1)$ persisted for 1 year. Females and current smokers had an increased risk for nonpersistence and non-adherence, while older age, overweight, receiving antihypertensive medication or colchicine, and suffering from dementia, diabetes and dyslipidaemia decreased the risk.

\section{Conclusion}

Medication adherence among gout patients on allopurinol therapy is poor, particular among females, younger, and those with less comorbidities. 
\begin{tabular}{l|l} 
Medication adherence among gout patients & 93
\end{tabular}

\section{INTRODUCTION}

Gout is a painful inflammatory condition, determined by the deposition of monosodium urate crystals within the joint and surrounding tissues. A worldwide prevalence of $0.6 \%$ has been described, but large variations across regions and sexes are acknowledged. ${ }^{1}$ In the UK, approximately $4 \%$ of men and $1 \%$ of women are affected. ${ }^{2}$ Both prevalence and incidence were significantly higher in 2012 than in 1997; with a 64\% increase in prevalence and $30 \%$ increase in incidence over this period. ${ }^{2}$ Gout and its main risk factor hyperuricemia have been associated with hypertension, cardiovascular disease, and chronic kidney failure. ${ }^{3}$ In the long-term, gout and its accompanied comorbidities can result in impaired function and ultimately a decline in health-related quality of life. ${ }^{4}$ In patients with recurrent gout flares or tophi, it is recommended to start long-term uric acid lowering therapy to reduce the number of gout flares and resolve tophi. ${ }^{5}$ Despite proven efficacy of these drugs, ${ }^{6,7}$ a substantial subgroup of patients fails to achieve optimal clinical benefit, partly because of poor medication adherence. Previous studies showed that non-adherence, usually defined as $80 \%$ or less of the total observation time that is covered by medication, is associated with higher serum uric acid concentrations and more gout flares. ${ }^{8-13}$ While non-persistence, occurrence of a gap in therapy use over time, may have little clinical effect in certain chronic diseases, a gap in uric acid lowering therapy might actually trigger or prolong a gout flare. ${ }^{12}$ Hence, poor medication adherence may therefore lead to a more severe disease for the patient and to an increase in gout-related healthcare costs for society. ${ }^{11,14}$

Recently, a systematic review confirmed poor medication adherence among gout patients using uric acid lowering therapy. ${ }^{15}$ Adherence ranged from $18 \%$ to $44 \%$ and persistence from $12 \%$ to $44 \% .{ }^{15}$ Twelve out of 16 studies included were conducted in the US, hampering transferability to a European setting where general practitioners are often the main healthcare provider. Data were often derived from electronic prescription records databases and managed health care plans, which only include insured residents and had, as a result, limited validity. Furthermore, most studies focused on nonadherence, but little is known about non-persistence, time until non-persistence, restart of therapy and subsequent medication adherence after first discontinuation. ${ }^{16}$ In order to identify patients at high risk, factors associated with poor medication adherence should be determined. Several factors such as older age or suffering from certain comorbidities like hypertension and diabetes, were associated with higher adherence rates $^{15}$ however, yet these factors have not or poorly been studied as determinants of non-persistence.

The objective of the current study was therefore to assess among newly diagnosed gout patients (i) rates of non-persistence and non-adherence with allopurinol therapy, (ii) determinants of non-persistence and non-adherence, and (iii) to describe the number of patients restarting therapy and subsequent medication adherence after the first occurrence of a gap in therapy. 
94 Chapter 5

\section{METHODS}

A retrospective cohort study was conducted using the UK Clinical Practice Research Datalink (CPRD), including more than 11.3 million individuals from 674 general practices in England, Northern Ireland, Scotland and Wales, representing $6.9 \%$ of the British population. ${ }^{17}$ The database provides detailed information on demographics, drug prescriptions, clinical events, specialist referrals, and hospital admissions. ${ }^{17}$ For this study we used data from 1987 through June 2014.

\section{Study population}

The study population consisted of individuals aged 40 years or older, with a first ever Read code of gout and a prescription of allopurinol during the period of valid data collection. The date of the first prescription of allopurinol after start of valid data collection defined the index-date. Follow-up was defined from the index-date to either the end of data collection ( $30^{\text {th }}$ of June 2014), the date of transfer of the patient out of the practice area, or the patient's death, whichever came first. Patients who switched to other uric acid lowering therapies (probenecid or febuxostat) during follow-up were censored for analyses. Patients with a prescription of uric acid lowering therapy prior to the diagnosis of gout and those who had a follow-up less than 90 days after completion of the first prescription were excluded from analyses.

\section{Measures of medication adherence}

Non-persistence was defined as the occurrence of a gap of at least 30 or 90 -days after the end of each prescription, in the case of overlap between two prescriptions (i.e. a repeat prescription within the duration of use of a previous prescription), the overlap days was added to the duration of treatment time. In case of a missing length, the median value of all allopurinol prescriptions was assigned. Non-persistence rate was measured by calculating the proportion of patients who discontinued treatment, a gap of at least 30-days or 90-days, over time.

Non-adherence was defined by the proportion of days covered (PDC). This was calculated as the number of days of prescribed medication divided by the total duration of follow-up, while truncating the possible overlap between two prescriptions. A PDC $<0.80$ was considered as non-adherence. Additionally, the medication possession ratio (MPR) was determined, calculated as number of days medication prescribed divided by the total duration of follow-up. In case of overlap between two prescriptions the overlap will be added to the number of days prescribed, this could lead to a MPR >1.0.

To improve insight into medication patterns, the proportion of patients restarting allopurinol therapy after the occurrence of a first 90-day gap was calculated. Subsequently, non-persistence estimates and non-adherence was once described in those patients restarting allopurinol therapy after a 90-day gap. 
\begin{tabular}{l|l} 
Medication adherence among gout patients & 95
\end{tabular}

\section{Potential determinants of non-adherence and non-persistence}

Several determinants were explored for their association with non-persistence and nonadherence. These factors related first to patient characteristics at baseline including gender, age (40-49, 50-59, 60-69, 70-79, and $\geq 80$ years), body mass index (<20.0, 20.0-24.9, 25.0-29.9, 30.0-34.9, and $\geq 35.0 \mathrm{~kg} / \mathrm{m}^{2}$ ), smoking status (never, current, former), alcohol consumption (yes, no), socio-economic status (low, low-medium, medium, medium-high, high), year start allopurinol (1987-1999, 2000-2005, 2006-2009, and 2010-2014 year), and the number of days between gout diagnosis and initiation of allopurinol. Additionally, the number of visits to general practitioners 12 months prior to the index date was considered (0, 1-9, 10-19, 20-29, $\geq 30$ visits).

Second, comorbidities prior to the index date were considered, including alcoholism, chronic obstructive pulmonary disease (COPD), dementia, depression, diabetes mellitus, hypertension, ischemic heart disease, myocardial infarction, osteoarthritis, renal calculi, and stroke. In the case of multiple records, the record most prior to the index-date was used. In addition, renal function was evaluated by reviewing laboratory test data (estimated glomerular filtration rate (eGFR) [MDRD] where possible), and CPRD read codes which describe the stage of renal function. In the case of multiple eGFR values on the same day the mean value was used. CPRD read codes were prioritized if there was a laboratory test on the same day.

Third, medication prescriptions six months prior to the index-date for hypertension, use of statins (a proxy indicator for dyslipidaemia), and acute gout medication (non-steroidal anti-inflammatory drugs [NSAIDs], colchicine, and oral corticosteroids) were considered.

\section{Statistical analysis}

Descriptive statistics were used to characterize the study population. Kaplan-Meier life table analyses were used to present non-persistence (30-day or 90-day gap) estimates with a $95 \%$ confidence intervals (CI) for 1 and 5 years after initiation of allopurinol. In addition, the median number of days until non-persistence was calculated. In a second analysis, Kaplan-Meier life table was applied to estimate the proportion of patients restarting allopurinol therapy after first 90-day gap. In addition, the median number of days until restarting allopurinol therapy and subsequent medication adherence were once more calculated.

Cox-regression analyses were performed to study the strength of the association between determinants with non-persistence (90-day gap), by entering all covariates into the regression model. The proportional hazards assumption was tested by including time interaction terms into the model. In case of violation ( $P$-value $<0.05)$, hazard ratios for the association between that covariate and non-persistence were calculated for the first year after the index date. Multivariable logistic-regression analyses were performed to study the strength of the association between determinants with non-adherence (PDC $<0.80$ ). 
All analyses were conducted using SAS software (Version 9.3; SAS Institute Inc., Cary, North Carolina).

\section{RESULTS}

\section{Study population}

Of the 131,565 newly diagnosed gout patients a total of 48,438 (38.8\%) patients initiated allopurinol as the first uric acid lowering therapy during observation period. Of the 48,438 allopurinol users, 158 patients were excluded for analyses because followup was shorter than 90 days after completion of the first prescription, resulting in a study population of 48,280 gout patients.

Table 1 shows the general characteristics of the study population. The mean age of the study population was 64.6 (SD 13.2) years and $76 \%$ were male. Comorbidities were common, the most common being hypertension (51\%), dyslipidaemia (30\%), osteoarthritis (24\%), and depression (16\%), and $41 \%$ received antihypertensive medication.

Table 1. Baseline characteristics of allopurinol users with gout $(n=48,280)$

\begin{tabular}{lc}
\hline Male & $75.7(36,169)$ \\
Age (years), mean \pm SD & $64.6 \pm 13.2$ \\
$40-49$ years & $16.1(7,750)$ \\
$50-59$ years & $21.3(10,305)$ \\
$60-69$ years & $24.2(11,684)$ \\
$70-79$ years & $23.6(11,375)$ \\
$80+$ years & $14.8(7,166)$ \\
BMI most recent prior to index date & \\
BMI (kg/m $\left.{ }^{2}\right)$ mean \pm SD & $29.7 \pm 5.4$ \\
$<20.0$ & $1.1 \%(536)$ \\
$20.0-24.9$ & $14.4(6,969)$ \\
$25.0-29.9$ & $38.1(18,393)$ \\
$30.0-34.9$ & $25.5(12,311)$ \\
$\geq 35.0$ & $13.4(6,467)$ \\
Missing & $7.5(3,604)$ \\
Smoking status & \\
Never & $42.6(20,573)$ \\
Current & $12.1(5,865)$ \\
Ex & $43.5(20,998)$ \\
Missing & $1.8(844)$ \\
Alcohol use & \\
No & $15.1(7,276)$ \\
Yes & $78.0(37,637)$ \\
Missing & $7.1(3,367)$ \\
Alcoholism & $4.5(2,150)$ \\
\hline
\end{tabular}


Table 1. (continued)

\begin{tabular}{|c|c|}
\hline \multicolumn{2}{|l|}{ Calendar year start allopurinol, index date } \\
\hline 1987-1999 & $17.5(8,430)$ \\
\hline $2000-2005$ & $29.1(14,071)$ \\
\hline $2006-2009$ & $32.0(15,464)$ \\
\hline $2010-2014$ & $21.4(10,315)$ \\
\hline Days between gout diagnosis and initiation allopurinol, mean \pm SD & $605 \pm 1039$ \\
\hline 0 & $24.3(11,717)$ \\
\hline $1-90$ & $26.7(12,903)$ \\
\hline $91-365$ & $14.1(6,789)$ \\
\hline$>366$ & $34.9(16,871)$ \\
\hline \multicolumn{2}{|l|}{ Number of GP visits in year prior to index date } \\
\hline 0 & $18.8(9,062)$ \\
\hline $1-9$ & $21.9(10,576)$ \\
\hline $10-19$ & $22.7(10,975)$ \\
\hline $20-30$ & $17.1(8,268)$ \\
\hline$\geq 30$ & $19.5(9,399)$ \\
\hline \multicolumn{2}{|l|}{ Socio-economic status } \\
\hline Low & $13.6(6,557)$ \\
\hline Low-medium & $14.9(7,177)$ \\
\hline Medium & $11.9(5,760)$ \\
\hline Medium - high & $10.3(4,973)$ \\
\hline High & $8.0(3,875)$ \\
\hline Missing & $41.3(19,938)$ \\
\hline \multicolumn{2}{|l|}{ History of drug use within 6 months before index date } \\
\hline Antihypertensive & $40.6(19,362)$ \\
\hline \multicolumn{2}{|l|}{ Acute gout treatment } \\
\hline Colchicine & $22.2(10,714)$ \\
\hline Corticosteroid & $7.6(3,647)$ \\
\hline NSAIDs & $67.0(32,332)$ \\
\hline \multicolumn{2}{|l|}{ History of comorbidity ever before index date } \\
\hline COPD & $15.1(7,296)$ \\
\hline Dementia & $0.5(250)$ \\
\hline Depression & $15.5(7,480)$ \\
\hline Diabetes & $10.3(4,950)$ \\
\hline Dyslipidaemia* & $30.2(14,603)$ \\
\hline Hypertension & $51.4(24,830)$ \\
\hline Ischemic heart disease & $17.7(8,561)$ \\
\hline Myocardial infarction & $8.9(4,304)$ \\
\hline Osteoarthritis & $23.8(11,507)$ \\
\hline Renal calculi & $1.1(517)$ \\
\hline Stroke & $6.4(3,111)$ \\
\hline \multicolumn{2}{|l|}{ Most recent eGFR measurement } \\
\hline$\left(\mathrm{ml} / \mathrm{min} / 1.73 \mathrm{~m}^{2}\right)$, mean $\pm \mathrm{SD}$ & $66.5 \pm 22.2$ \\
\hline CKD 1 & $12.9(6,212)$ \\
\hline CKD 2 & $38.6(18,644)$ \\
\hline CKD 3 & $35.8(17,299)$ \\
\hline CKD 4 & $2.9(1,420)$ \\
\hline CKD 5 & $0.3(141)$ \\
\hline Missing & $9.5(4,564)$ \\
\hline
\end{tabular}

Data are presented as percentage and number $(n)$ unless otherwise indicated. Abbreviations: BMl: body mass index; CKD: chronic kidney disease; GP: general practitioner; NSAIDs: non-steroidal anti-inflammatory drug; COPD: chronic obstructive pulmonary disease; eGFR: estimated glomerular filtration rate. CKD 1: eGFR 90; CKD 2: eGFR 60<90; CKD 3: eGFR 30<60; CKD 4: eGFR 15<30; CKD 5: eGFR<15 
$98 \mid$ Chapter 5

\section{Medication adherence}

Non-persistence estimates for treatment with allopurinol are displayed in Figure 1. Considering a gap-length of 30-days, non-persistence with allopurinol therapy increased from $57.8 \%(95 \% \mathrm{Cl} 57.3-58.2)$ at year 1 to $80.9 \%(95 \% \mathrm{Cl} 80.4-81.2)$ at 5 years following initiation (Table 2). The median time until discontinuation was 225 days (95\% $\mathrm{Cl} 220-231)$. Increasing the gap length up to 90-days non-persistence estimates were $38.5 \%$ (95\% Cl 38.1-38.9) at year 1 and $56.9 \%$ (95\% Cl 56.4-57.4) at 5 years following initiation, with a median time until discontinuation of 1029 days (95\% Cl 988-1078).

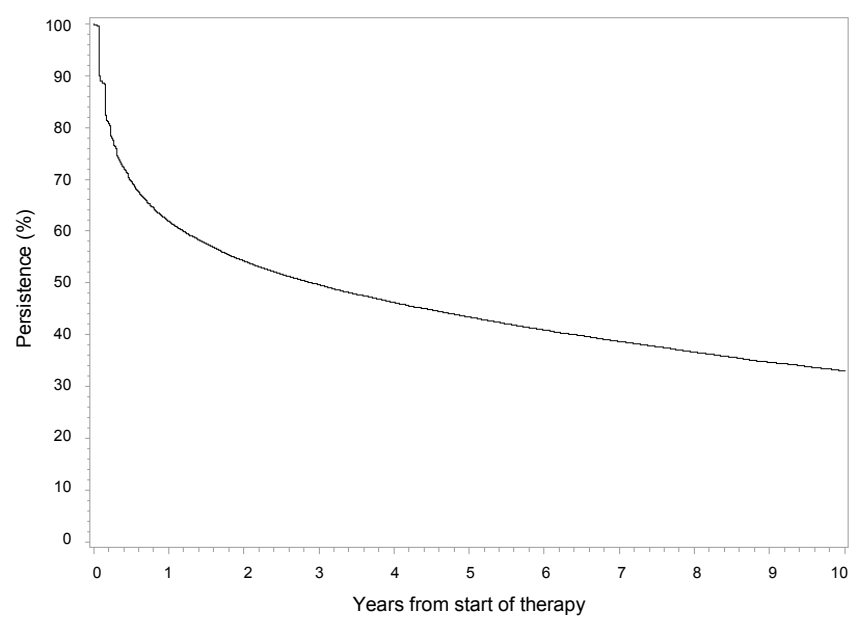

Figure 1. Kaplan-Meier curves for persistence to treatment with allopurinol medication in a the total study population

The vast majority of patients were non-adherent to allopurinol therapy (61\%). The PDC was on average 0.57 (SD 0.43), indicating that patients had an allopurinol prescription for $57 \%$ of the observed days. The MPR was on average 0.66 , and $47.6 \%$ of the patients had an MPR $>0.80$ and $23.2 \%$ even reached a MPR $>1.00$.

Of the 26,235 patients who experienced a 90-day gap, 15,013 (57\%) restarted allopurinol therapy. Of all patients who had a gap of at least 90 -days, $43.3 \%(95 \% \mathrm{Cl}$ 42.7-43.9) restarted therapy within 1 year, and this increased to $64.2 \%(95 \% \mathrm{Cl}$ 63.5-64.9) within 5 years (Figure 2). Median time until restart was 643 days $(95 \% \mathrm{Cl}$ 617-678). Among patients who restarted therapy, $75.7 \%(95 \% \mathrm{Cl} 75.0-76.4)$ experienced a 30 -day gap and $52.3 \%(95 \% \mathrm{Cl} 51.4-53.1)$ experienced a 90-day gap in the first year after re-initiation. Here, the median time until discontinuation was 88 days (95\% Cl 84-97) for a 30-day and 319 days (95\% Cl 301-340) for a 90-day gap (Figure 3). After restarting allopurinol, the average PDC was 0.49 (SD 0.31) and only $10.3 \%$ were considered adherent. 
Table 2. Medication adherence among gout patients initiated allopurinol $(n=48,280)$

\begin{tabular}{|c|c|c|c|c|}
\hline \multirow{2}{*}{$\begin{array}{l}\text { Non-persistence* } \\
\text { First year }\end{array}$} & \multicolumn{2}{|c|}{ 30-day gap } & \multicolumn{2}{|c|}{ 90-day gap } \\
\hline & 57.8 & $(57.3-58.2)$ & 38.5 & $(38.1-38.9)$ \\
\hline First five years & 80.8 & $(80.4-81.2)$ & 56.9 & $(56.4-57.4)$ \\
\hline Time until discontinuation (days), median $(95 \% \mathrm{Cl})$ & 225 & $(220-231)$ & 1029 & $(988-1078)$ \\
\hline \multicolumn{5}{|l|}{ Adherence (PDC) } \\
\hline Mean \pm SD & \multicolumn{2}{|c|}{$0.57 \pm 0.34$} & & \\
\hline Median (IQR) & \multicolumn{2}{|c|}{$0.67(0.65)$} & & \\
\hline \multicolumn{5}{|l|}{ Categories, n (\%) } \\
\hline$<0.20$ & \multicolumn{2}{|l|}{23.1} & \multicolumn{2}{|l|}{$(11,136)$} \\
\hline $0.20-0.40$ & \multicolumn{2}{|l|}{10.9} & \multicolumn{2}{|l|}{$(5,242)$} \\
\hline $0.40-0.60$ & \multicolumn{2}{|l|}{11.9} & \multicolumn{2}{|l|}{$(5,756)$} \\
\hline $0.60-0.80$ & \multicolumn{2}{|l|}{15.8} & \multicolumn{2}{|l|}{$(7,611)$} \\
\hline $0.80-1.00$ & \multicolumn{2}{|l|}{38.4} & \multicolumn{2}{|l|}{$(18,535)$} \\
\hline \multicolumn{5}{|c|}{ Estimates for restarting therapy after a gap of at least 90-days } \\
\hline First year & \multicolumn{2}{|l|}{43.3} & \multicolumn{2}{|c|}{$(42.7-43.9)$} \\
\hline First five years & \multicolumn{2}{|l|}{64.2} & \multicolumn{2}{|c|}{$(63.5-64.9)$} \\
\hline Time until restart (days), median $(95 \% \mathrm{Cl})$ & \multicolumn{2}{|l|}{643} & \multicolumn{2}{|c|}{$(617-678)$} \\
\hline \multicolumn{5}{|l|}{ Medication adherence after restarting therapy $(n=14,084)$} \\
\hline Non-persistence* & \multicolumn{2}{|c|}{ 30-day gap } & \multicolumn{2}{|c|}{ 90-day gap } \\
\hline First year & 75.7 & $(75.0-76.4)$ & 52.3 & $(51.4-53.1)$ \\
\hline First five years & 91.1 & $(90.5-91.7)$ & 71.6 & $(70.7-72.5)$ \\
\hline Time until $2^{\text {nd }}$ discontinuation (days), median (95\% Cl) & 87 & $(84-94)$ & 313 & $(294-334)$ \\
\hline \multicolumn{5}{|l|}{ Adherence (PDC) } \\
\hline Mean \pm SD & \multicolumn{2}{|c|}{$0.49 \pm 0.31$} & & \\
\hline \multicolumn{5}{|l|}{ Adherence (PDC $\geq 0.80$ ) } \\
\hline no & \multicolumn{2}{|l|}{76.8} & \multicolumn{2}{|l|}{$(10,819)$} \\
\hline yes & \multicolumn{2}{|l|}{23.2} & $(3,265)$ & \\
\hline
\end{tabular}

Data are presented as percentage and number $(n)$ unless otherwise indicated. *Kaplan-Meier estimates for nonpersistence at (\%) at different time periods following initiation, by 30-day and 90-day gap lengths. Cl: confidence interval; IQR: interquartile range; PDC: proportion days covered; SD: standard deviations.

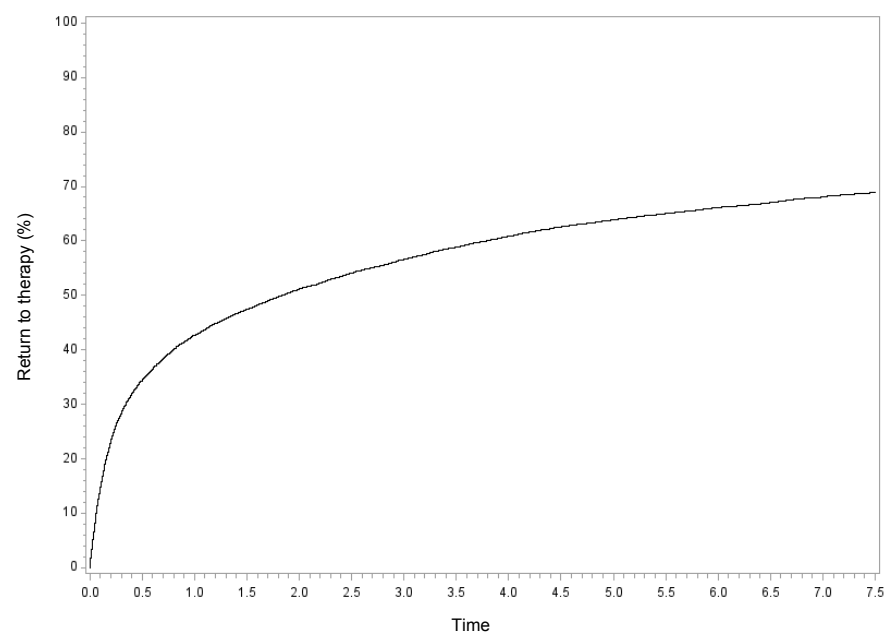

Figure 2. Kaplan-Meier curve for cumulative incidence of restart with allopurinol medication after first gap of 90 days. 


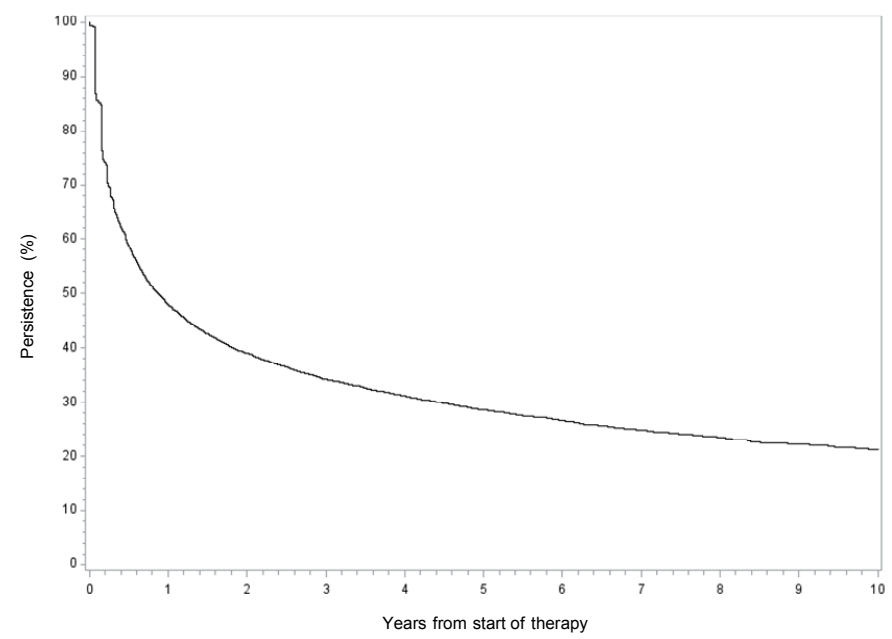

Figure 3. 1 - Kaplan-Meier curve for persistence to treatment with allopurinol medication after restarting therapy after a first 90-day gap.

\section{Factors associated with medication adherence}

Factors associated with non-persistence or non-adherence are presented in Table 3. In the multivariable adjusted model, female gender and current smoking status increased the risk of non-persistence and non-adherence. While older age, overweight, former smoking, use of colchicine, and suffering from dementia, diabetes mellitus, or dyslipidaemia decreased the risk of non-persistence and non-adherence.

The effect of use of antihypertensive medication, year start allopurinol, and kidney function on non-persistence, however, was not constant over time ( $p$ value interaction $<0.05$ ). When follow-up was restricted to the first 365 days following initiation, the use of antihypertensive medication decreases the risk to of non-persistence (HR: $0.73 ; 95 \%$ $\mathrm{Cl}$ 0.70-0.77). Patients starting allopurinol between 2010 and 2014 were more likely to be persistent compared to patients who started between 1987 and 1999 (HR: 0.96; 95\% $\mathrm{Cl}$ 0.87-0.99). Kidney function was not significantly associated with non-persistence in the first year after initiation. 


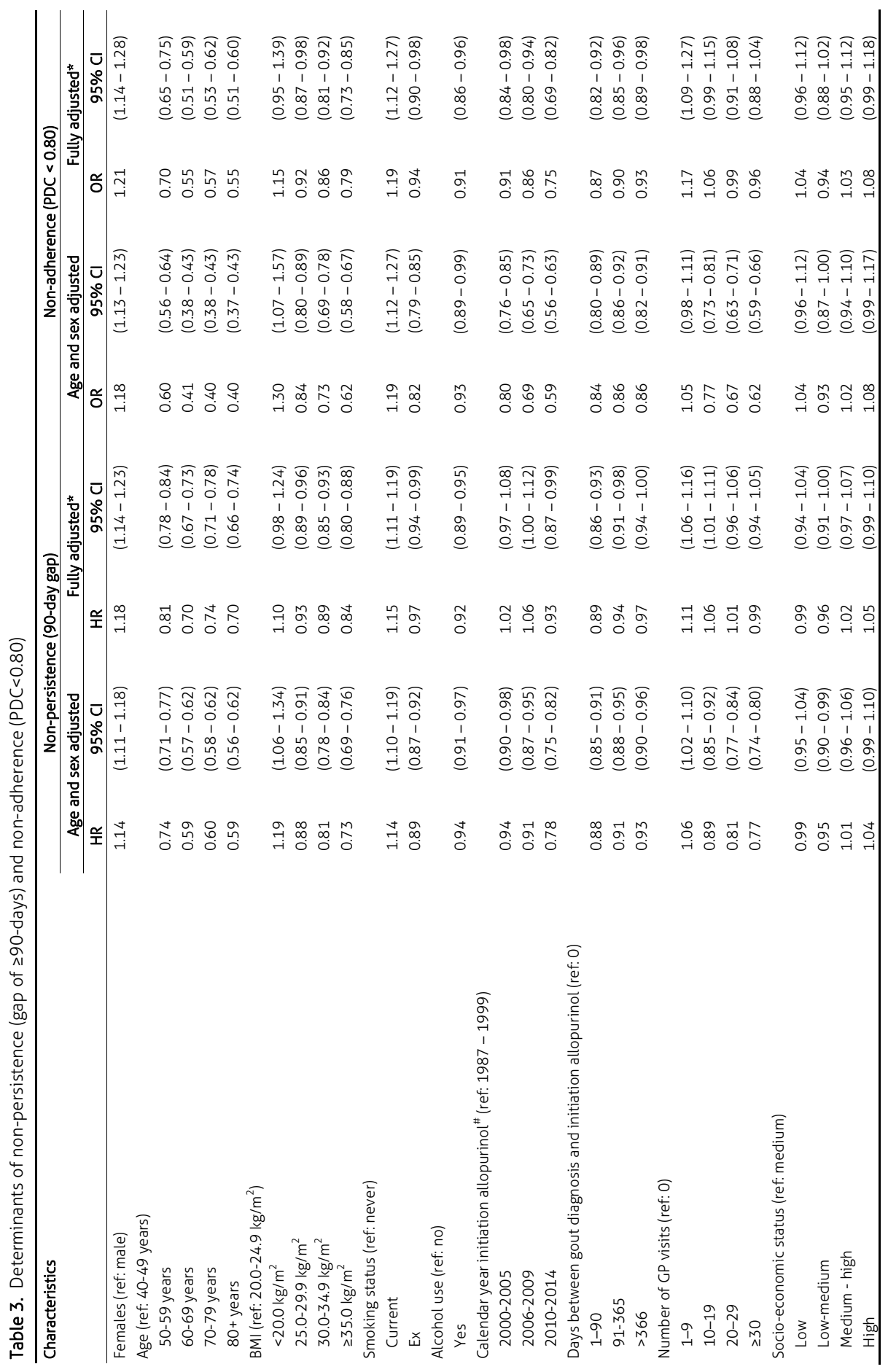


$102 \mid$ Chapter 5

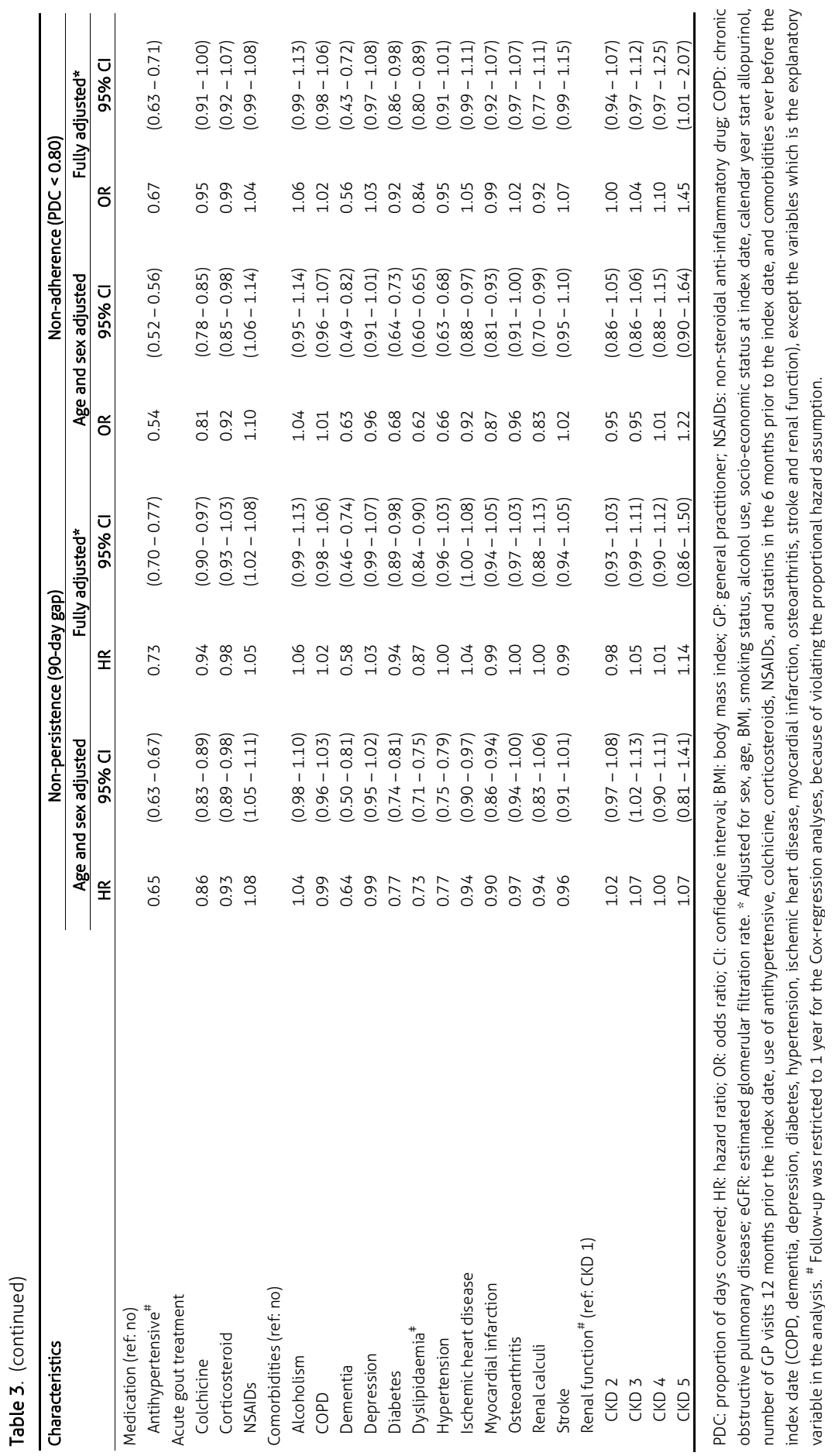




\section{DISCUSSION}

The present study showed poor medication taking behaviour among incident gout patients initiated allopurinol. Non-persistence (90-day gap) with allopurinol therapy increased from $57 \%$ at 1 year to $77 \%$ at 5 years following initiation. After the occurrence of a gap 54\% of the patients restarted therapy; yet less than half of the patients persisted with treatment for 1 year following restarting therapy. During the entire observation period only $38 \%$ of the patients were considered adherent.

Non-persistence estimates found in the present study were comparable to the few previous conducted studies, which showed rates between $56 \%$ and $88 \%{ }^{16,18-20}$ All studies used pharmacy-claims data and three out of four were conducted in the USA. $^{16,19,20}$ Despite these differences in population and design, it seems that the variation in non-persistence rates mainly depends on the permissible gap-length and observation period. For example, Solomon et al. showed among mainly female ( $80 \%)$ and American gout patients that $56 \%$ experienced a gap of at least 30-days in the first year after starting allopurinol. ${ }^{20}$ Another study conducted among 15,908 Irish gout patients found that $81 \%$ experienced a gap of 5 weeks and $54 \%$ a gap of 9 weeks in the first year after initiation of uric acid lowering therapies. ${ }^{18}$

The dynamics in medication use is less frequently studied, such as whether patients return to therapy after the occurrence of a gap and if they remain on therapy after restarting. We found that almost half of the patients returned to therapy after a 90-day gap in the first year. This finding is in line with a study conducted by Harrold et al. who showed that $70 \%$ had a gap of at least 60-days in therapy and among those with a gap an estimated $50 \%$ returned to treatment within 8 months, and by 4 years, $75 \%$ had restarted. ${ }^{16}$ Although it is encouraging that many patients return to therapy after the occurrence of a first gap, we found that only 50\% remained on therapy in the first year after reinitiating. Multiple and extended gaps in therapy lead to less efficiency in achieving the target level of serum uric acid, required to dissolve existing monosodium urate crystals and to prevent further gout flares. ${ }^{21}$

In contrast to persistence, adherence is a measure of medication adherence during the entire observation period. In the present study only $38 \%$ of the gout patients were considered adherent. Although, our reliance on a PDC of more than 0.80 to be considered adherent is arbitrary, the mean PDC was 0.57, which is far from optimal. In 2012, a systematic review reported mean adherence rates all below 0.80 and the proportion of adherent patients ranged from $10-46 \%{ }^{15}$ This was based on 10 claims/electronic records of which nine were conducted in the US. ${ }^{15}$ Recently, two European studies have been conducted. Among Irish patients $35.8 \%$ had an MPR $\geq 0.80$ in the first 12 months, using pharmacy claims data. ${ }^{18}$ In an Italian study the rates were even more alarming, $10 \%$ of the 3727 gout patients were adherent to allopurinol in the first 150 days and just $3 \%$ in the first year. ${ }^{10}$ Our data from the UK and the studies conducted in Ireland ${ }^{18}$ and Italy, ${ }^{10}$ indicate that poor medication adherence among gout patients not only occurs in patients from the US but also in Europeans. 
$104 \mid$ Chapter 5

To improve medication adherence, patients at-risk should be identified. In our study, females were more prone to have poor medication adherence; this is in line with other previous research, ${ }^{13,18}$ while others found the opposite, ${ }^{8,22,23}$ or no effect of gender on persistence or adherence. ${ }^{10,20}$ In agreement with our findings, older patients and those being overweight ${ }^{13}$ and suffering from comorbidities such as, diabetes, and hypertension were more likely to be persistent and adherent. ${ }^{8,10,13,19,22,24}$ Although not investigated, these patients appear to be less healthy and might have more severe gout or are aware of the negative consequences of poor medication adherence on their health than younger and healthier gout patients. ${ }^{11,19,20,25}$ For example, we showed that users of antihypertensive medication had a $27 \%$ lower hazard to be non-persistent (90-day gap) and a $33 \%$ lower odds to be non-adherent, compared to non-users. Suffering from overweight appears to decrease the risk by approximately $11 \%$ and $14 \%$, for dyslipidaemia $13 \%$ and $16 \%$, and for diabetes $6 \%$ and $8 \%$, to become non-persistence and non-adherence, respectively. Somewhat contra-intuitively, persons registered with dementia had a lower risk of non-persistence (HR: 0.58) and non-adherence (OR: 0.56), most likely because they will be supervised by taking their medication. Likely, general practitioners register dementia only in the clear and more severe cases. Therefore, it cannot be excluded that person with mild dementia also have a lower risk for poor medication adherence.

The reason for poor medication adherence among gout patients is complex and is probably also attributable to poor illness perception and incorrect medication beliefs. ${ }^{26-}$ 29 Herein, the care provider can contribute by explaining the pathophysiology of the disease, the short- and long-term side effects of urate lowering therapy and the role of life style and diet changes. Nevertheless, the awareness and support from the health providers in medication adherence in those with gout is far from optimal. ${ }^{30}$ Up to now, there is only one intervention study in gout patients which investigated the effect of nurse delivered 'package of care', including patient education, lifestyle advice and uric acid lowering therapy, on medication adherence and the recommended treatment target of serum uric acid $<360 \mu \mathrm{mol} / \mathrm{L}$. More than $90 \%$ of the gout patients $(n=106)$ succeeded in achieving the therapeutic target during 12 months of follow-up. ${ }^{31}$ Furthermore, excellent adherence and persistence of four years after the responsibility of treatment is taken over by the patient's general practitioner was achieved. ${ }^{32}$ Emphasizing that, as in other chronic diseases, nurse delivery of care is likely to be effective in improving medication adherence and achieving therapeutic targets. ${ }^{32-34}$

This study represents a comprehensive analysis of medication adherence among newly diagnosed gout patients from a representative primary care data-set from the UK. There were certain limitations to this study. First, medication adherence was estimated retrospectively by analysis of allopurinol prescribed by the general practitioner. Therefore, we could not ascertain if patients actually purchased and took the prescribed medication. On the other hand, by using prescription data any distortion caused by patient recall or desire to give social accepted answers can be eliminated ${ }^{35}$ and is an acceptable and accurate measure of persistence and adherence. ${ }^{36}$ Second, the reason 
for stopping or interrupting allopurinol use could not be traced and might be physiciandirected. Since the chronic nature of the disease and the low prevalence of the sideeffects we do not expect big shifts in our findings. Third, only patients initiated allopurinol were included. A low number of patients switching to probenecid or febuxostat were censored. Likely these drugs are prescribed to patients with different health characteristics, and might receive more attention in the healthcare system influencing their adherence to medication. Third, adherence was defined as a PDC of $\geq 0.80$. Even though the dichotomization of adherence is widely used in the literature, this cut-off is arbitrary and not clinically validated.

In conclusion, our study showed poor medication adherence among incident gout patients initiated allopurinol. The results of the present study further add to the literature, that although patients experiencing a gap in therapy are likely to return, the changes of a backlash are as likely. Highlighting the need for additional research focusing on improving medication adherence among those initiated allopurinol therapy, but also among those restarting therapy after the occurrence of a gap. Clear guidelines and studies investigating means to improve medication adherence are urgently needed to tackle this complex problem. Target groups for intervention may be those who are younger and considered healthier, so with a normal weight and those not on antihypertensive medication and suffering from comorbidities like dementia, depression, dyslipidaemia, and hypertension. 


\section{REFERENCES}

1. Wijnands JM, Viechtbauer W, Thevissen K, et al. Determinants of the prevalence of gout in the general population: a systematic review and meta-regression. Eur J Epidemiol. 2015; 30(1):19-33.

2. Kuo CF, Grainge MJ, Mallen C, et al. Rising burden of gout in the UK but continuing suboptimal management: a nationwide population study. Ann Rheum Dis. 2015; 74(4):661-667.

3. Feig DI, Kang DH, Johnson RJ. Uric acid and cardiovascular risk. N Engl J Med. 2008; 359(17):1811-1821.

4. Wertheimer A, Morlock R, Becker MA. A revised estimate of the burden of illness of gout. Curr Ther Res Clin Exp. 2013; 75:1-4.

5. Richette P, Doherty M, Pascual E, et al. 2016 updated EULAR evidence-based recommendations for the management of gout. Ann Rheum Dis. 2017; 76(1):29-42.

6. Shoji A, Yamanaka $H$, Kamatani N. A retrospective study of the relationship between serum urate level and recurrent attacks of gouty arthritis: evidence for reduction of recurrent gouty arthritis with antihyperuricemic therapy. Arthritis Rheum. 2004; 51(3):321-325.

7. Schumacher HR Jr., Becker MA, Wortmann RL, et al. Effects of febuxostat versus allopurinol and placebo in reducing serum urate in subjects with hyperuricemia and gout: a 28-week, phase III, randomized, double-blind, parallel-group trial. Arthritis Rheum. 2008; 59(11):1540-1548.

8. Rashid N, Levy GD, Wu YL, et al. Patient and clinical characteristics associated with gout flares in an integrated healthcare system. Rheumatol Int. 2015; 35(11):1799-1807.

9. Rashid N, Coburn BW, Wu YL, et al. Modifiable factors associated with allopurinol adherence and outcomes among patients with gout in an integrated healthcare system. J Rheumatol. 2015; 42(3):504-512.

10. Mantarro S, Capogrosso-Sansone A, Tuccori M, et al. Allopurinol adherence among patients with gout: an Italian general practice database study. Int J Clin Pract. 2015; 69(7):757-765.

11. Halpern R, Mody RR, Fuldeore MJ, et al. Impact of noncompliance with urate-lowering drug on serum urate and gout-related healthcare costs: administrative claims analysis. Curr Med Res Opin. 2009; 25(7):1711-1719.

12. Park H, Rascati KL, Prasla K, et al. Evaluation of health care costs and utilization patterns for patients with gout. Clin Ther. 2012; 34(3):640652.

13. Zandman-Goddard G, Amital H, Shamrayevsky N, et al. Rates of adherence and persistence with allopurinol therapy among gout patients in Israel. Rheumatology (Oxford). 2013; 52(6):11261131.

14. Wu EQ, Patel PA, Yu AP, et al. Disease-related and all-cause health care costs of elderly patients with gout. J Manag Care Pharm. 2008; 14(2):164-175.

15. De Vera MA, Marcotte $G$, Rai S, et al. Medication adherence in gout: A systematic review. Arthritis Care Res (Hoboken). 2014.

16. Harrold LR, Andrade SE, Briesacher B, et al. The dynamics of chronic gout treatment: medication gaps and return to therapy. Am J Med. 2010; 123(1):54-59.

17. Herrett E, Gallagher AM, Bhaskaran K, et al. Data Resource Profile: Clinical Practice Research Datalink (CPRD). Int J Epidemiol. 2015;44(3):827836.

18. McGowan B, Bennett K, Silke C, et al. Adherence and persistence to urate-lowering therapies in the Irish setting. Clin Rheumatol. 2014.

19. Sarawate CA, Brewer KK, Yang W, et al. Gout medication treatment patterns and adherence to standards of care from a managed care perspective. Mayo Clin Proc. 2006; 81(7):925934.

20. Solomon DH, Avorn J, Levin R, et al. Uric acid lowering therapy: prescribing patterns in a large cohort of older adults. Ann Rheum Dis. 2008; 67(5):609-613.

21. Pascual $E_{1}$ Sivera F. Time required for disappearance of urate crystals from synovial fluid after successful hypouricaemic treatment relates to the duration of gout. Ann Rheum Dis. 2007; 66(8):1056-1058.

22. Riedel AA, Nelson M, Joseph-Ridge $N$, et al. Compliance with allopurinol therapy among managed care enrollees with gout: a retrospective analysis of administrative claims. J Rheumatol. 2004; 31(8):1575-1581.

23. Horsburgh S, Norris P, Becket $G$, et al. Allopurinol use in a New Zealand population: prevalence and adherence. Rheumatol Int. 2014; 34(7):963970.

24. Lee S, So MW. Adherence with urate-lowering therapies among male patients with gout in a routine clinical setting. Mod Rheumatol. 2016: 16. 
25. Harrold LR, Andrade SE, Briesacher BA, et al. Adherence with urate-lowering therapies for the treatment of gout. Arthritis Res Ther. 2009; 11(2):R46.

26. Harrold LR, Mazor KM, Velten S, et al. Patients and providers view gout differently: a qualitative study. Chronic Illn. 2010; 6(4):263271.

27. Singh JA. Facilitators and barriers to adherence to urate-lowering therapy in African-Americans with gout: a qualitative study. Arthritis Res Ther. 2014; 16(2):R82.

28. Spencer K, Carr A, Doherty M. Patient and provider barriers to effective management of gout in general practice: a qualitative study. Ann Rheum Dis. 2012; 71(9):1490-1495.

29. Harrold LR, Mazor KM, Peterson D, et al. Patients' knowledge and beliefs concerning gout and its treatment: a population based study. BMC Musculoskelet Disord. 2012; 13:180.

30. Spaetgens B, Pustjens T, Scheepers LE, et al. Knowledge, illness perceptions and stated clinical practice behaviour in management of gout: a mixed methods study in general practice. Clin Rheumatol. 2016.

31. Rees F, Jenkins W, Doherty M. Patients with gout adhere to curative treatment if informed appropriately: proof-of-concept observational study. Ann Rheum Dis. 2013; 72(6):826-830.

32. Abhishek A, Jenkins W, La-Crette J, et al. Longterm persistence and adherence on uratelowering treatment can be maintained in primary care-5-year follow-up of a proof-ofconcept study. Rheumatology (Oxford). 2017.

33. Griffiths C, Foster G, Barnes N, et al. Specialist nurse intervention to reduce unscheduled asthma care in a deprived multiethnic area: the east London randomised controlled trial for high risk asthma (ELECTRA). Brit Med J. 2004; 328(7432):144-147.

34. Welch G, Garb J, Zagarins S, et al. Nurse diabetes case management interventions and blood glucose control: Results of a meta-analysis. Diabetes Res Clin Pr. 2010; 88(1):1-6.

35. Choo PW, Rand CS, Inui TS, et al. Validation of patient reports, automated pharmacy records, and pill counts with electronic monitoring of adherence to antihypertensive therapy. Med Care. 1999; 37(9):846-857.

36. Lau HS, de Boer A, Beuning KS, et al. Validation of pharmacy records in drug exposure assessment. J Clin Epidemiol. 1997; 50(5):619625. 



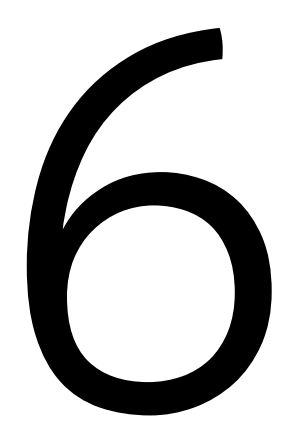

Medication adherence among patients with gout: a systematic review and meta-analysis 


\section{ABSTRACT}

\section{Objective}

In the management of chronic gout, a large proportion of patients need long-term management with urate lowering therapy (ULT). This study reviews medication adherence to ULT and summarizes factors associated with adherence.

\section{Methods}

We performed a systematic literature search for studies on adherence to ULT among gout patients in PubMed, Embase, CINAHL, and PsycINFO. We conducted meta-analysis, with a random effect model, for the studies reporting the proportion of patients considered adherent to at least $80 \%$ of prescribed medication or time taken. We explored potential sources of heterogeneity, including geographic area and measure of adherence. Narrative summaries were made for data on adherence assessed/defined by Medication Event Monitoring System (MEMS)/pill-count or patient-reported, occurrence of a gap in therapy $\geq 30$ days (non-persistence), and factors associated with adherence.

\section{Results}

Of the 23 studies, 15 assessed adherence using prescription/claims data, two by the MEMS or pill count, and six by patient-reported data. The pooled proportion of adherent patients ( $n=13)$ was $46.0 \%$ (95\% Cl 40.8-51.2); $45 \%$ across studies conducted in the USA $(n=8)$ and $48 \%$ in other countries $(n=5)$. Adherence assessed by MEMS/pill count and patient-reported was much higher than by studies using prescription/claims data. Non-persistence $(n=5)$ ranged from $56 \%$ to $87 \%$. Factors associated with adherence were investigated in 18 studies, strong evidence for a positive association of older age, more comorbidities, and the presence of diabetes or hypertension was found.

\section{Conclusion}

Medication adherence to ULT among gout patients was poor. Better insight into reasons and consequences or poor adherence is needed. 


\section{INTRODUCTION}

Gout is the most common type of inflammatory arthritis, affecting approximately 1-3\% of the population worldwide. ${ }^{1,2}$ Hyperuricemia, the main risk factor for gout, can be successfully treated with urate lowering therapy (ULT). The initiation of ULT is recommended in patients with recurrent gout attacks or with tophi, as these might lead to debilitating long-term consequences such as joint damage. ${ }^{3}$ Despite availability of effective treatments, a substantial proportion of patients experiences multiple gout flares and develops tophi, signifying insufficient control of the disease. ${ }^{4}$ One of the barriers towards optimal dug treatment of gout is poor medication adherence of the patient to ULT.

Medication adherence refers to the process by which patients take their medications as prescribed. $^{5}$ It is a complex construct and contains several components, including adherence and non-persistence. Adherence is usually defined as the extent to which a patient's actual medication intake corresponds to the prescribed dosing regimen during observation time. Non-persistence is defined as the occurrence of a gap in taking therapy (e.g. for at least 30- or 60-days), or stopping.

In 2014, a systematic review of 16 studies by De Vera et al. revealed poor overall medication adherence to ULT among gout patients. ${ }^{6}$ The proportion of patients considered adherent ranged from $56 \%$ to $72 \%$. Non-persistence ranged from $56 \%$ to $88 \% .^{6}$ However, 12 of 16 studies were conducted in the USA, hampering transferability of results to the European setting. Furthermore, data were often derived from electronic prescription records and managed health care plans, which might have introduced selection bias and misclassification. Since then, several new studies were published, also from outside the USA and using others methods than prescription/claim data to assess medication adherence.

The aims of the present study were (i) to perform a systematic review and meta-analysis on studies that assess medication adherence and/or non-persistence among gout patients treated with ULT, (ii) to perform subgroup analyses on demographical and methodological characteristics that might explain heterogeneity, and (iii) to present an overview of studies addressing the association of medication adherence with explanatory variable factors and with gout related clinical outcomes.

\section{METHODS}

A systematic literature review was conducted of primary studies investigating medication adherence to ULT in patients with gout. The search was performed in PubMed, Embase, PsycINFO, and CINAHL databases (inception to August $15^{\text {th }} 2016$ ) and combined free text- and keyword-search terms relating to gout and hyperuricemia, ULT, and medication adherence (Appendix 1: search strategy). In addition, a hand search of references of relevant articles was performed. Studies not published in English language, 
letters, editorials, reviews, case reports, opinion articles, congress abstracts and randomized controlled trials were excluded. The latter are assumed to not reflect accurately real-world patterns of patient medication adherence. For final inclusion, studies had to meet the following criteria: (i) presenting primary data from an original research study; (ii) concerning patients with evidence of gout or hyperuricemia; (iii) being prescribed or taking ULT; and (iii) reporting on at least one measure of medication adherence. No restrictions on the methods to assess medication adherence was made.

A two-stage screening process was performed. First, titles, index terms, and/or abstracts were screened to identify potentially relevant articles. If in doubt, the full article was evaluated in the second step. Both steps were independently performed by two reviewers (LS and MO) using a standardized protocol and reporting form. Difference in assessment was resolved by arbitration of a third reviewer (AB), and consensus was reached after discussion.

\section{Data extraction}

Data extraction was performed by one reviewer using a standardized data extraction form (LS), and was checked by a second reviewer (MO). Data extraction included: study identification (first author, year of publication), study characteristics (study design, setting, recruitment site, country, inclusion period, inclusion criteria, sample size, observation time for adherence), patient characteristics (average age of patients, percentage of males), case definition of gout, type of ULT, and medication adherence.

With regard to the assessment of medication adherence the following information was extracted, the method for assessing medication adherence (using prescription/claims data, patient-reported etc.), the component of medication adherence studied (adherence, non-persistence, time until non-persistence etc.), the type of measurement used (medication possession ratio (MPR) [sum of days' supply / days of observation], proportion of days covered (PDC), which is more conservative that the MPR since a possible overlap between two prescriptions is truncated), ${ }^{7}$ and estimate and corresponding threshold to define the outcome (mean adherence, proportion of patients with a PDC or MPR of at least $80 \%$, gap of at least 30 days etc.).

\section{Quality assessment / risk of bias}

Quality scoring of observational studies is controversial because the proposed criteria may not be relevant for the study question or for the actual quality of the study. ${ }^{8}$ Moreover, the current review included 16 prescription/claims studies, which have a similar risk of bias with regard to generalisability, absence of information on drop-out, misclassification of disease and exposure and assessment of outcome. Therefore, we have chosen to discuss the results in the light of aspects related to risk of bias and factors which might influence medication adherence such as study population and the method to assess medication adherence. 


\section{Meta-analysis}

Since for the majority of studies the proportion of patients adherent to ULT was based on a threshold of $80 \%$, the results could be pooled by performing a meta-analysis. To ensure consistency in the interpretation of the outcome, the figures of studies reporting proportion non-adherent were converted to present proportion non-adherent to ULT. Heterogeneity was tested by Cochran's $\mathrm{O}$ test and the $P^{2}$ statistic, the latter describing the percentage of variation across studies. ${ }^{9}$ Whenever heterogeneity was high $\left(I^{2}>50 \%\right)$, random effects models were used. ${ }^{10}$ Potential sources of heterogeneity were further explored by stratification for (1) studies conducted in the USA opposed to other countries and (2) differences in the measure of adherence (PDC or MPR).

Further narrative summaries were provided for studies reporting adherence using another type of measure/numeric value and for the other outcomes, including nonpersistence, the association between medication adherence and factors and clinical gout related outcomes.

\section{Factors associated with medication adherence}

If available, information on factors associated with medication adherence was extracted from each study. Consistent with the World Health Organization (WHO) framework, factors were categorized into patient-, condition-, therapy-, social/economic-, and health care system factors. Factors were considered associated with medication adherence based on significance (P-value $<0.05$ ) and the direction of association was reported as being positively, negatively or no association. The strength of evidence across studies was classified into four levels of evidence, according to recommendations by Van Tulder et al. ${ }^{11}$ The level of evidence was summarized as follows: 1 ) strong: $\geq$ four studies and $\geq 75 \%$ of all studies considered report findings in the same direction; 2 ) moderate: three studies and two report findings in the same direction; 3 ) limited: only one or two studies and $\geq 75 \%$ of all studies reported findings in the same direction; 4 ) conflicting: $\geq 75 \%$ of the studies report inconsistent findings.

Furthermore, the association between medication adherence and gout related outcomes, including serum uric acid and the number of gout flares was registered.

\section{RESULTS}

The search resulted in 1991 potentially relevant articles (Figure 1). After excluding duplicates and screening of title, index terms, and/or abstract, 98 articles remained for full-paper review. After reading the full-text, a total of 23 articles were included. Hand search of references did not reveal additional articles. Because of the different methods to assess medication adherence, the characteristics of the studies were presented according to the type of method, i.e. prescription/claims, MEMS/pill count or patientreported data (Table 1 ). 


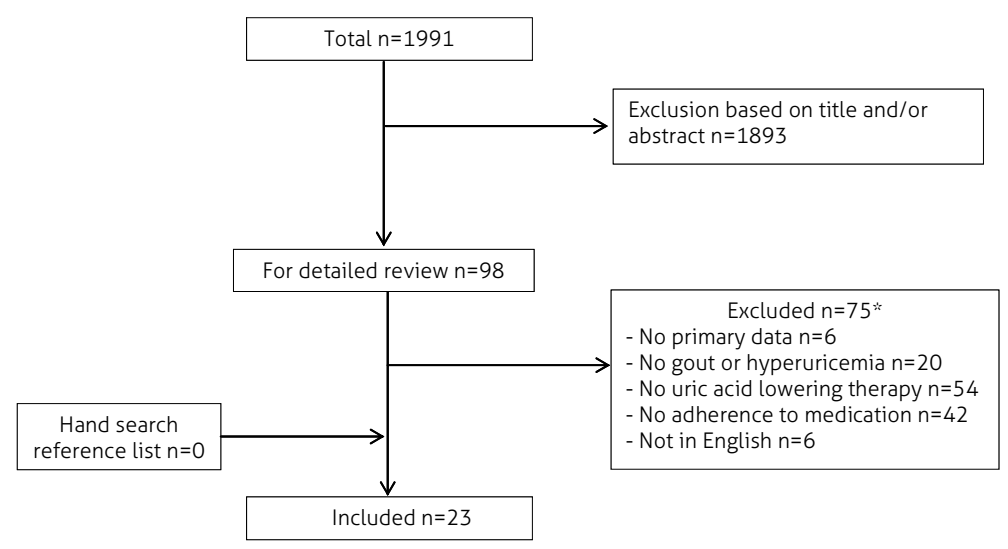

Figure 1. Flow-diagram of systematic literature search

* Numbers are not mutually exclusive

\section{Characteristics of included studies}

\section{Prescription/claims data}

Sixteen studies used prescription/claims data, 10 (64\%) were conducted in the USA, one in Israel, ${ }^{24}$ one in New Zeeland ${ }^{12}$ and three in Europe $\left(\mathrm{UK}_{1}{ }^{2}\right.$ Italy, ${ }^{13}$ and Ireland ${ }^{14}$ ). All of them made use of claims or pharmacy data, except the studies from Italy and UK which used records in primary care databases. Patients were classified as having gout by either a gout diagnosis (ICD- or read-codes) or a dispensing record for ULT. ${ }^{12,14}$ Study sample sizes ranged from $242^{15}$ to 49,395 patients. $^{2}$ Adherence was defined as either a MPR $(n=7)$ or PDC $(n=7)$ of more than $80 \%$.

\section{Electronic monitoring device or pill count}

Of the two studies retrieved, one was conducted in The Netherlands and used MEMS to assess mean adherence (number of patients=17). The diagnosis of gout was based on physician opinion. ${ }^{16}$ The other study, conducted in Korea, used pill count by the nurse to assess adherence, (number of patients=132). The diagnosis of gout was based on a physicians' assessment of the Wallace classification criteria. ${ }^{17}$ Adherence was defined as at least $80 \%$ of the prescribed dose was not returned. Both studies included patients from a Rheumatology centre. 


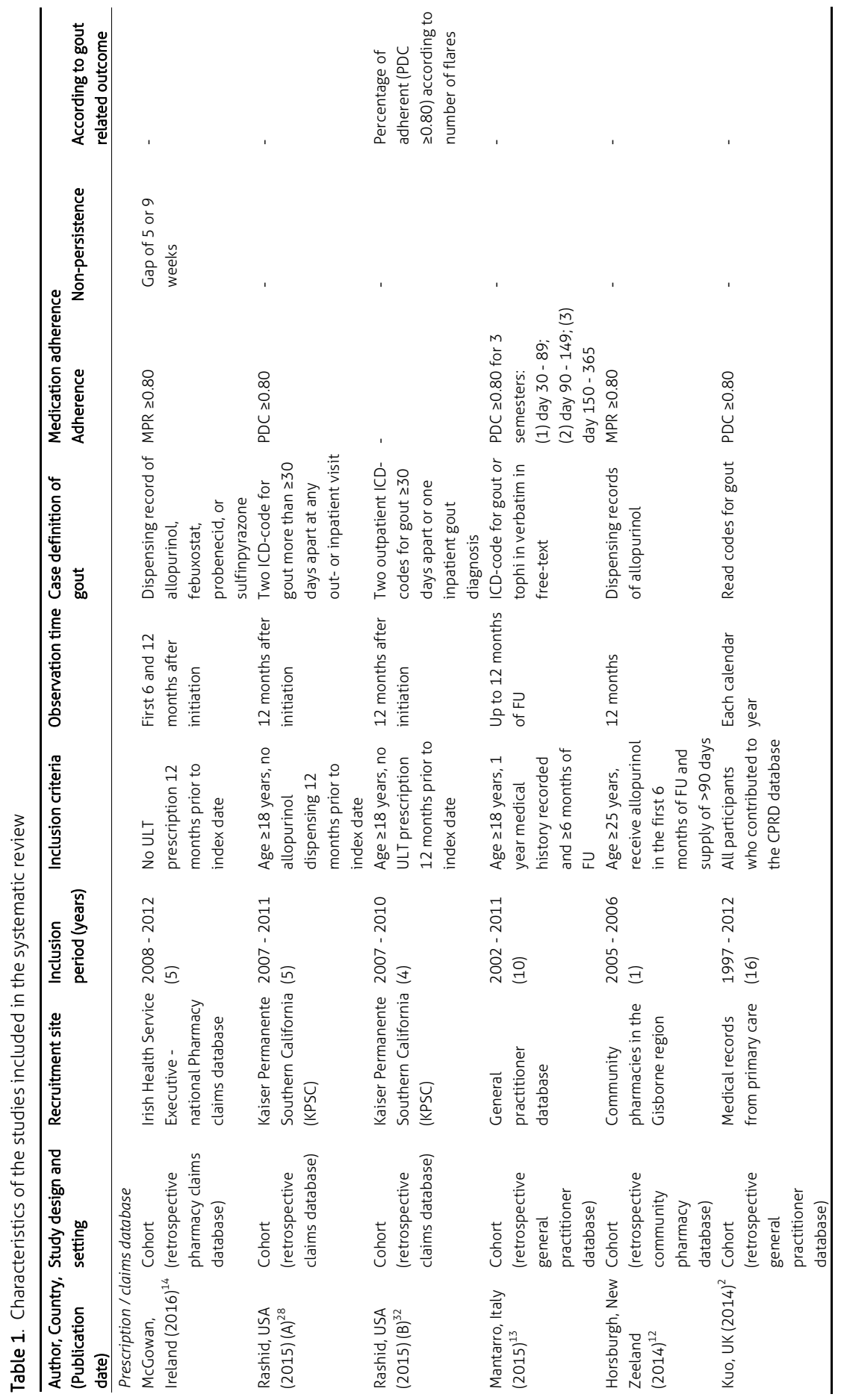


$116 \mid$ Chapter 6

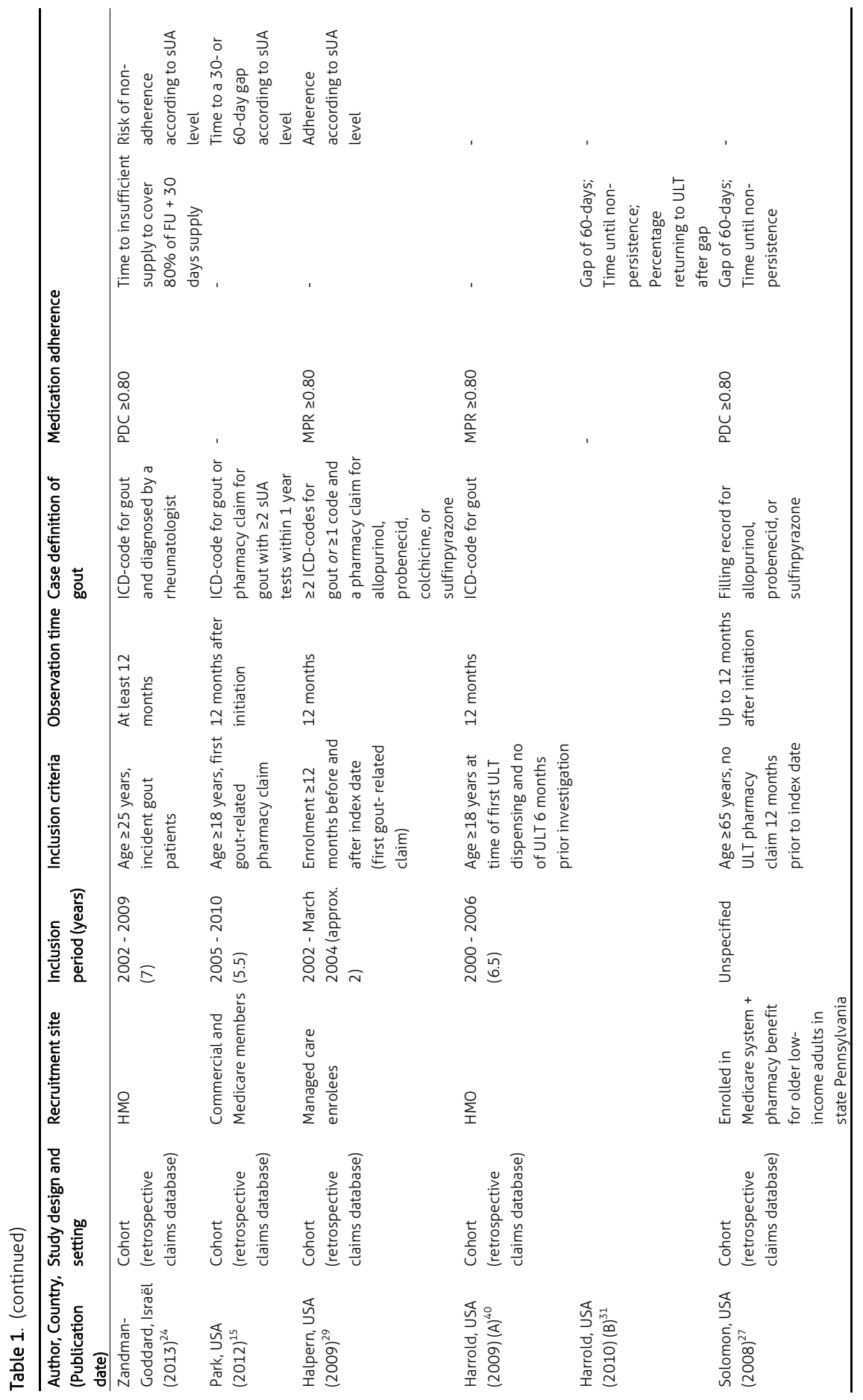




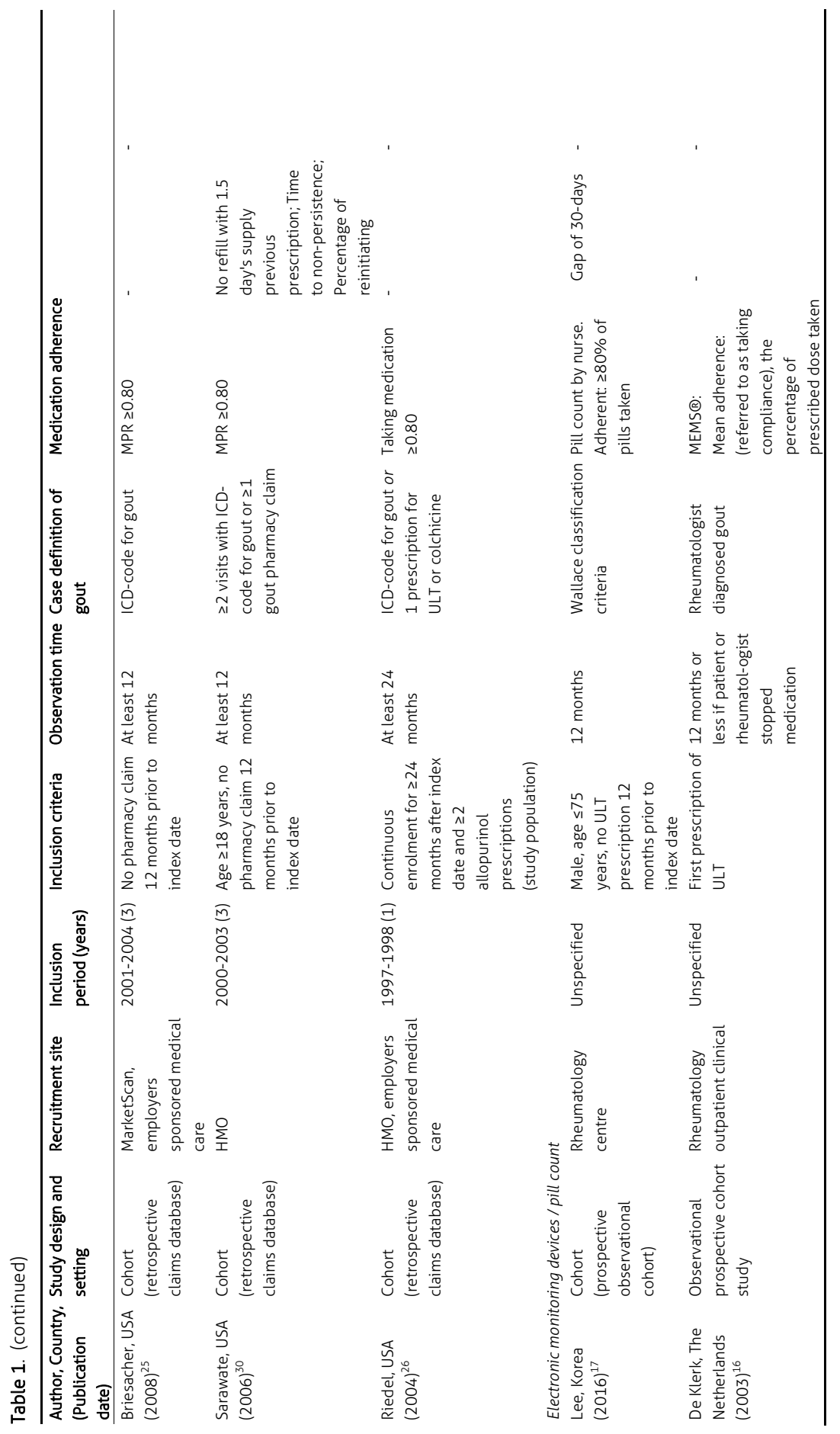


$118 \mid$ Chapter 6

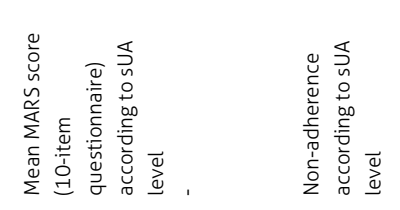

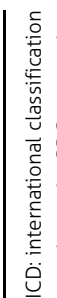

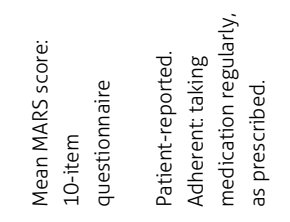

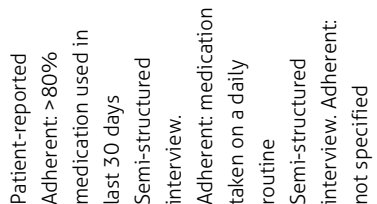

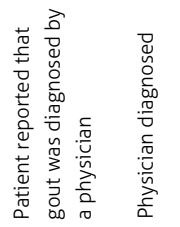

旁蒙品

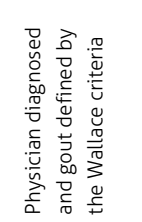

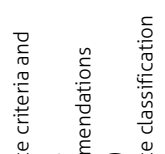

ᄃ을

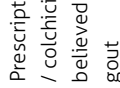

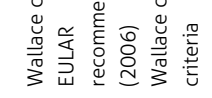

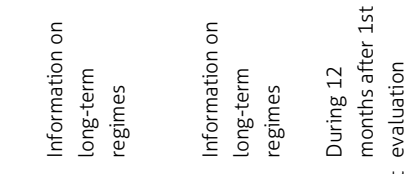

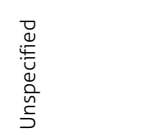

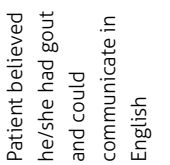

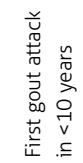

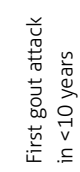

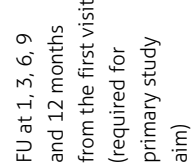

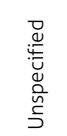

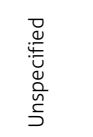

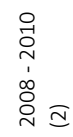

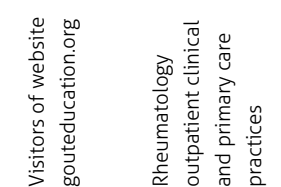

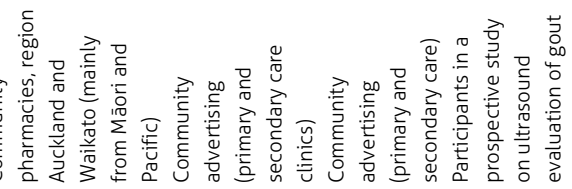

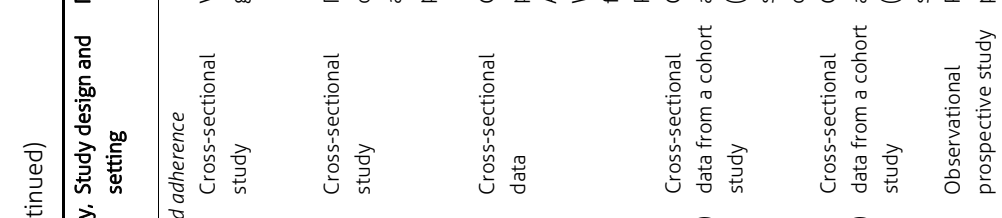

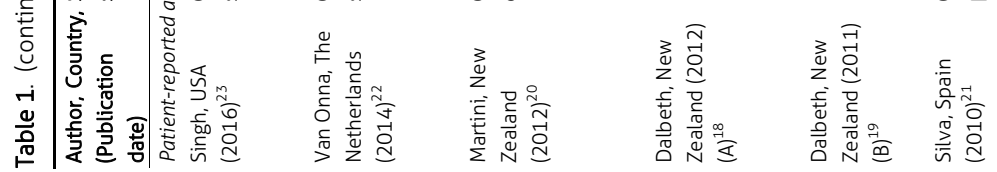

\begin{tabular}{|c|}
\hline 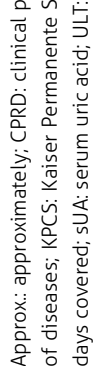 \\
\hline
\end{tabular}




\section{Patient-reported data}

Six studies used patient-reported data to assess medication adherence, three were conducted in New Zeeland, ${ }^{18-20}$ two in Europe (Spain ${ }^{21}$ and The Netherlands ${ }^{22}$ ), and on in the USA. ${ }^{23}$ The diagnosis of gout was either patient self-reported ${ }^{20,23}$ or physician reported. The latter could either be based on the physicians opinion ${ }^{22}$ or by using of the Wallace classification criteria. ${ }^{18,19,21}$ Sample sizes were considerably smaller, ranging from $15^{22}$ to $251 .^{23}$ To measure medication adherence, the two studies from New Zealand had administered the validated nine-item Medication Adherence Report Scale (MARS) questionnaire, which provides a mean adherence estimate. ${ }^{18,19}$ A higher score (max 45) represents better adherence. Adherence for the four remaining studies was reported as the proportion of patients indicating to take medication as a daily routine $(n=2),{ }^{21,22}$ taking at least $80 \%$ of their medication $(n=1),{ }^{23}$ or considered himself adherent (yes/no) $(n=1){ }^{20}$

\section{Medication adherence}

\section{Adherence}

In 13 studies the proportion of patients considered adherent was based on the threshold of being covered for at least $80 \%$ during observation time. Adherence ranged from $17 \%^{24}$ to $78 \%{ }^{12}$ (median $=37 \%{ }^{25}$ ) in 11 prescription/claims studies, was $71 \%{ }^{17}$ in the study using pills count by the nurse, and was $79 \%$ in the study using patient-reported adherence. $^{23}$ In the meta-analysis, the pooled proportion of patient adherent, in the random effects model was $46.0 \%$ (95\% confidence interval $(\mathrm{Cl})$ 40.8-51.2) with a substantial heterogeneity observed ( $\mathrm{Q}=3551, P$-value $<0.001, \mathrm{I}^{2}=99 \%$ ) (Table S1). Figure 2 shows a forest plot with the estimated proportion of patients considered adherent. In a stratified meta-analysis (Table S2), the pooled proportion of patients adherent was similar across studies conducted in the USA $(n=8 ; 45.2 \%, \mathrm{Cl} 39.8-50.6)$ and other countries $(n=5 ; 47.5 \%, \mathrm{Cl} 36.5-58.6)$. The pooled proportion of patients adherent was higher in studies using MPR $(n=6 ; 44.1 \%, \mathrm{Cl} 37.6-50.7)$ compared to studies using PDC $(n=4 ; 33.5 \%, C l 24.4-43.2)$. Three of the 13 studies did not specify the type of measure (PDC/MPR) and were therefore not included in the stratified meta-analysis according to PDC or MPR. ${ }^{17,23,26}$

Mean adherence was reported in seven studies and ranged from $54 \%{ }^{26}$ to $88 \%$. ${ }^{12,16,25-29}$ Because of the low number of studies presenting mean adherence and often missing data on the according standard deviations, we did not pool the results for mean adherence.

For the five remaining studies the proportion of patients adherent was not based on the threshold of $80 \%$, and were therefore not included in the meta=analysis. Adherence was defined as taking medication "on a daily routine", 22 "on a regular basis as prescribed" 21 and one study did not define when a patient was considered adherent. ${ }^{20}$ Adherence rates in these studies ranged from $40 \%{ }^{20}$ to $79 \% .{ }^{19}$ Finally, two studies used the MARS 
questionnaire to assess adherence, but only the mean scores were presented ranging from 39.0 (SD 7.4) to 4.2 (SD 2.7), 45 is considered highly adherent. ${ }^{18,19}$

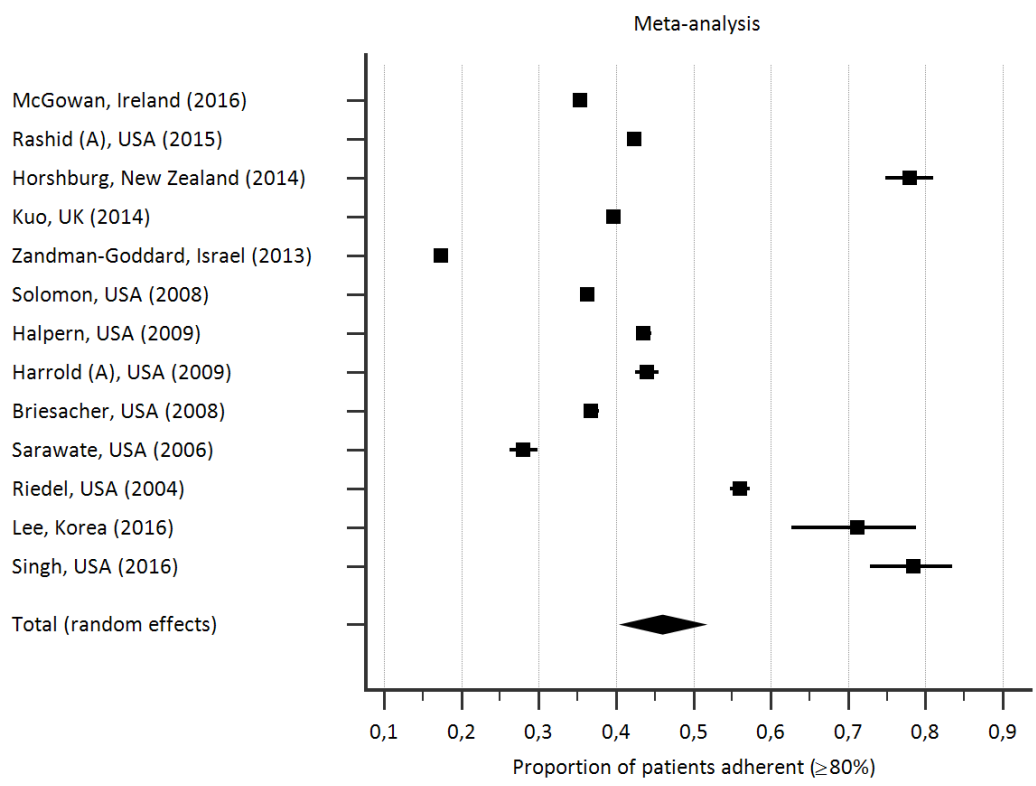

Figure 2. Forest plot: pooled estimate of proportion of patients adherent

Non-persistence

Five studies reported non-persistence, and rates ranged from $56 \%{ }^{27}$ to $87 \%{ }^{14,17,30,31}$ Time until non-persistence was investigated in three studies and ranged from 4 to 12 months. ${ }^{24,30,31}$ The variation in outcomes is mostly attributable to the differences in the permissible gap length and observation period (Table 2). Due to large differences in defining non-persistence and the low number of studies, it was inappropriate to create a pooled estimate.

\section{Factors associated with medication adherence}

Table 3 summarizes factors associated with medication adherence among 18 studies. We found strong evidence for better medication adherence in patients who are older, had a higher number of comorbidities, and suffered from diabetes or hypertension. In addition, patients from African-America and Mãori descent, were more likely to have poor medication adherence (moderate evidence). 12,27,28 Several factors were infrequently studied and therefore no conclusions on the role of factors as smoking status, body mass index, socio-economic status could be made. The same applies to other condition- or patient-related factors, such as perceived health status or understanding illness, these aspects were only investigated by single studies. ${ }^{16,17,19,23}$ 


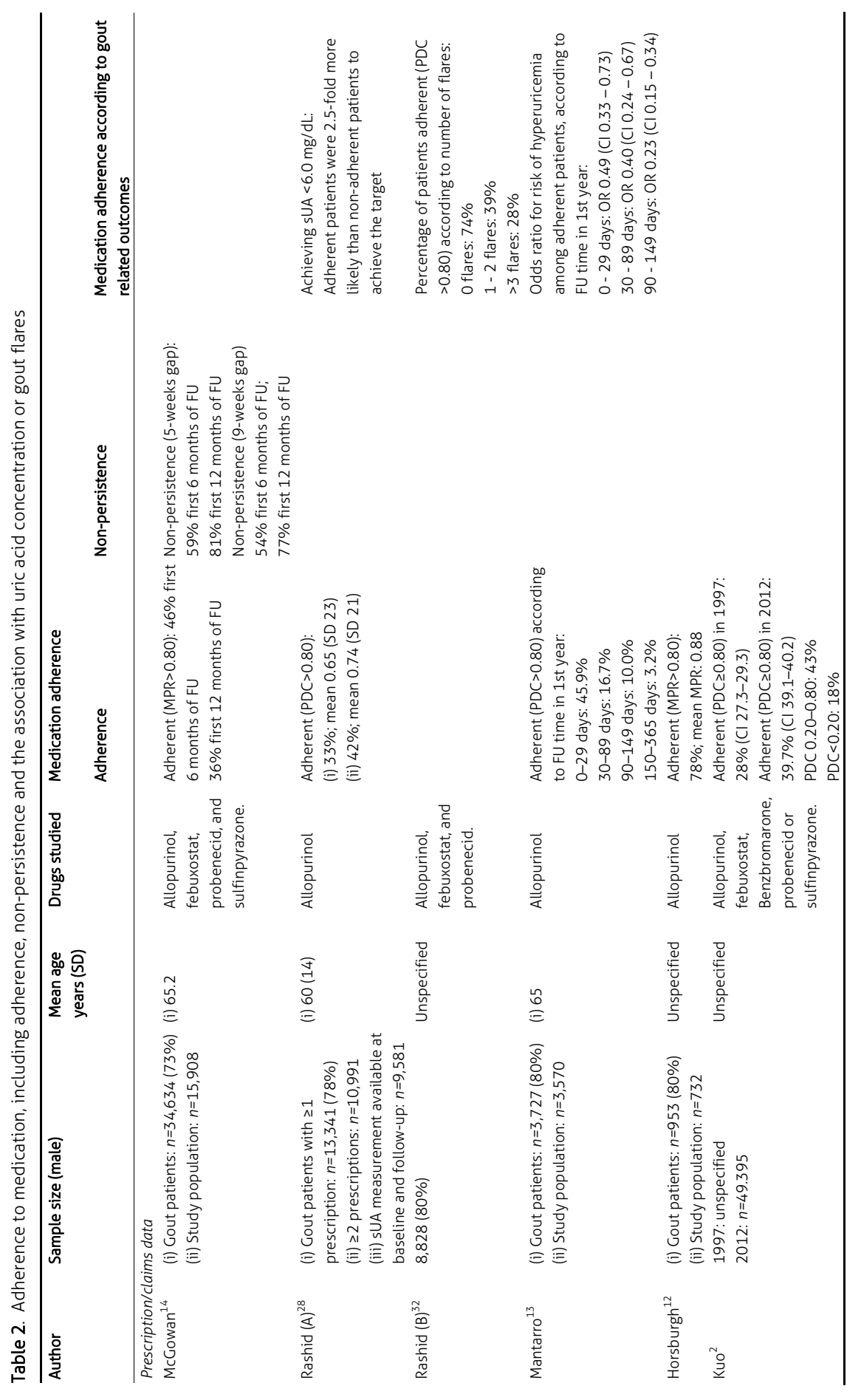


122 Chapter 6

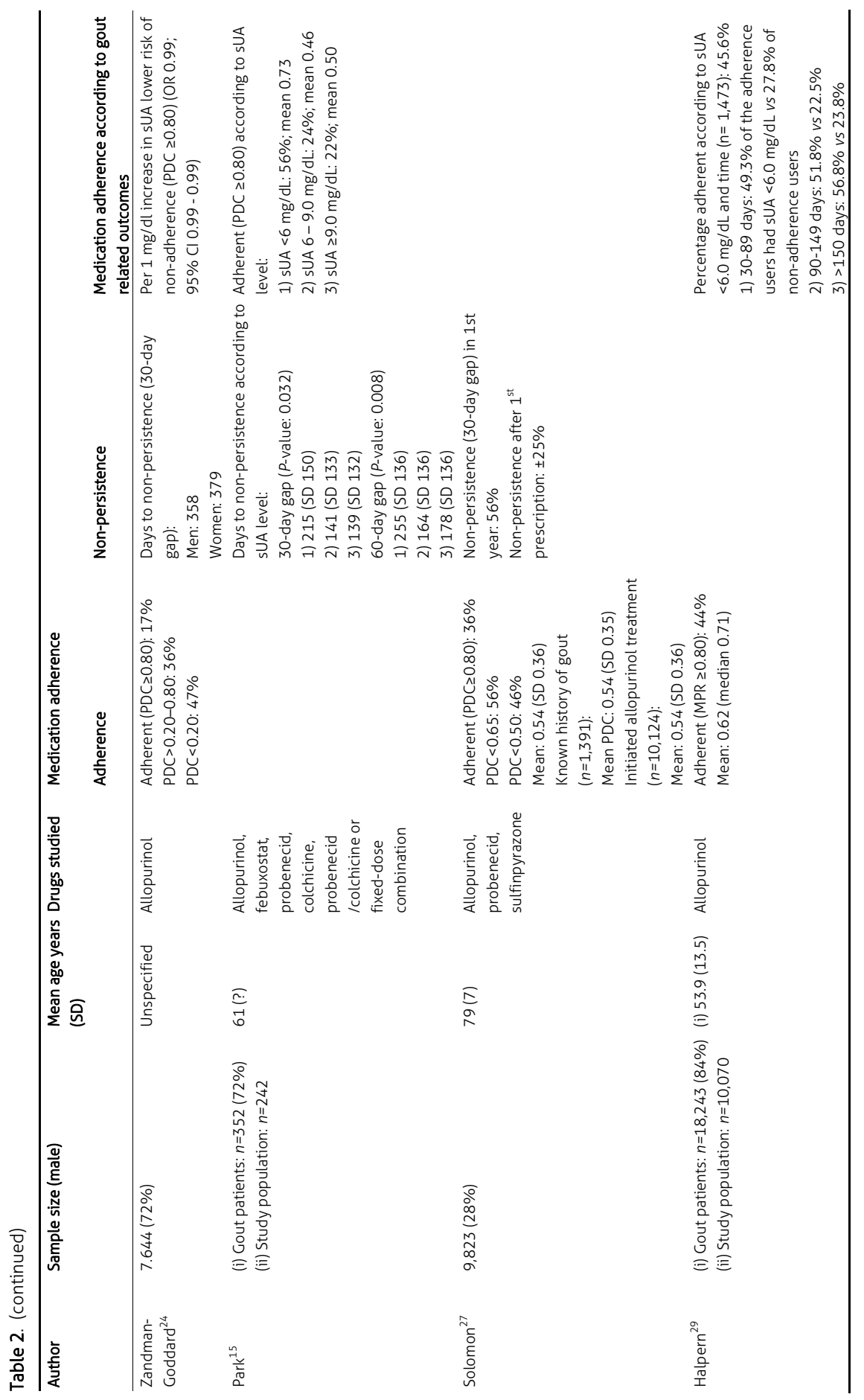




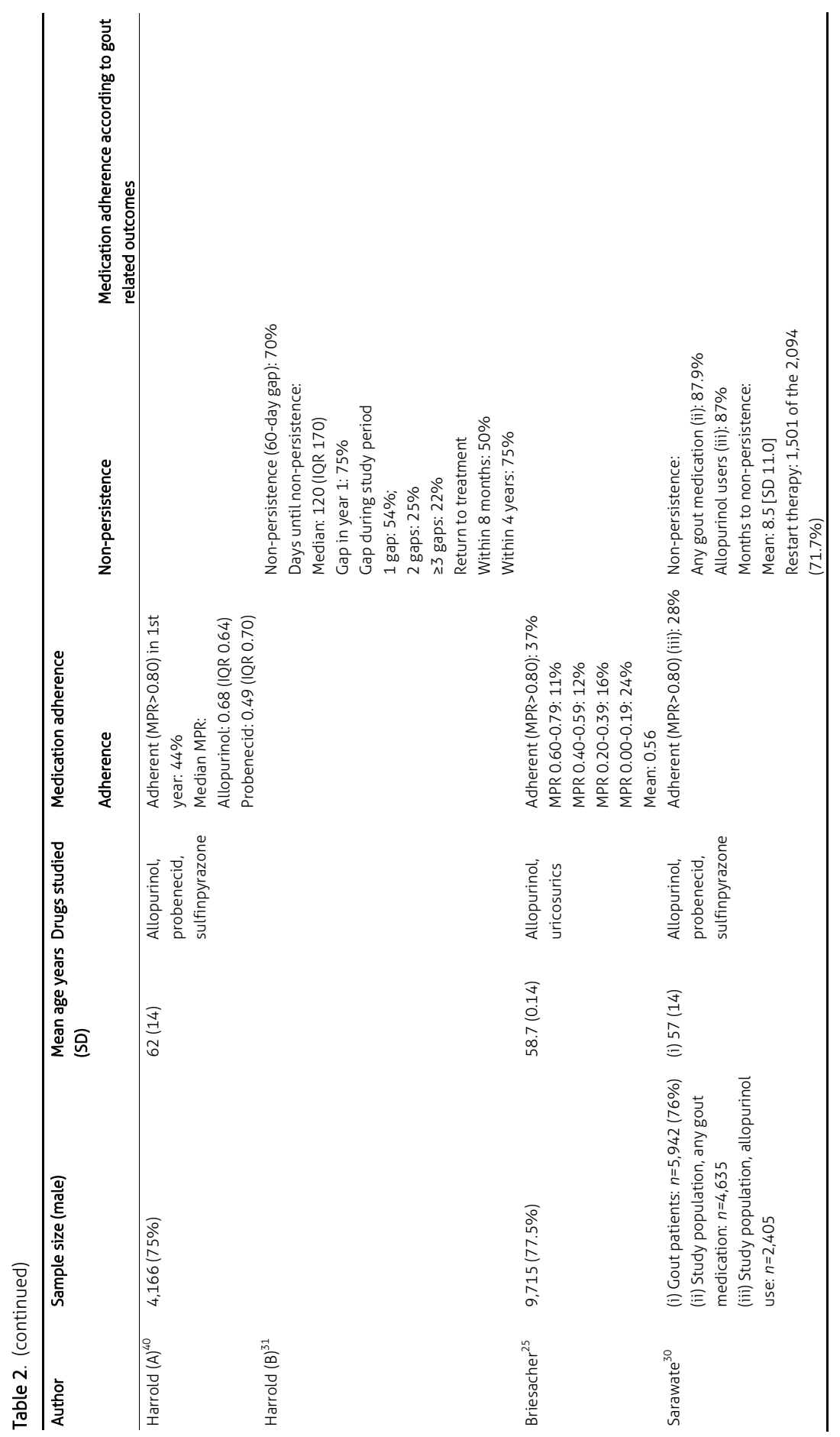


124 Chapter 6

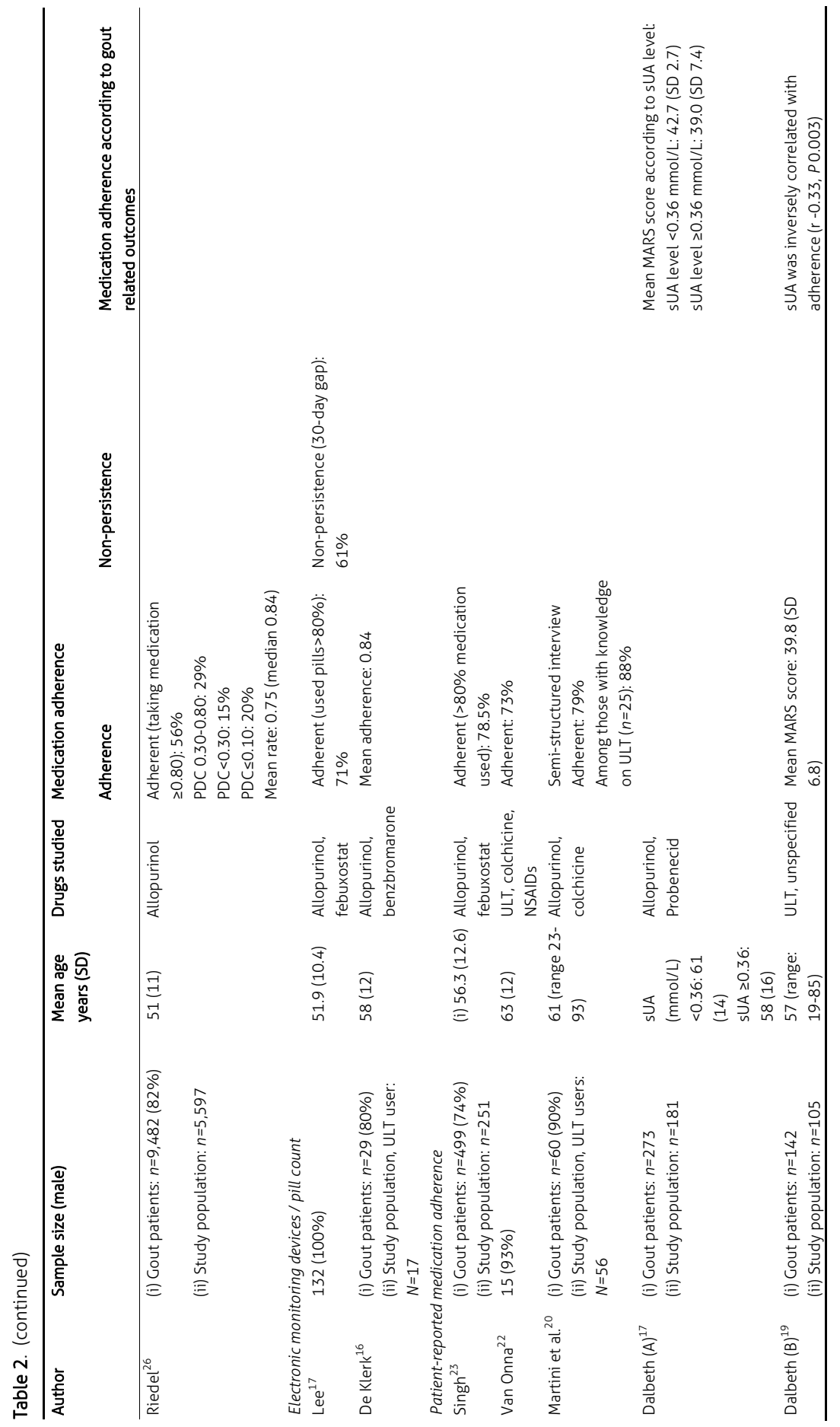


SLR and meta-analysis: medication adherence among gout patients $\mid \begin{aligned} & 125 \\ & \text { a }\end{aligned}$

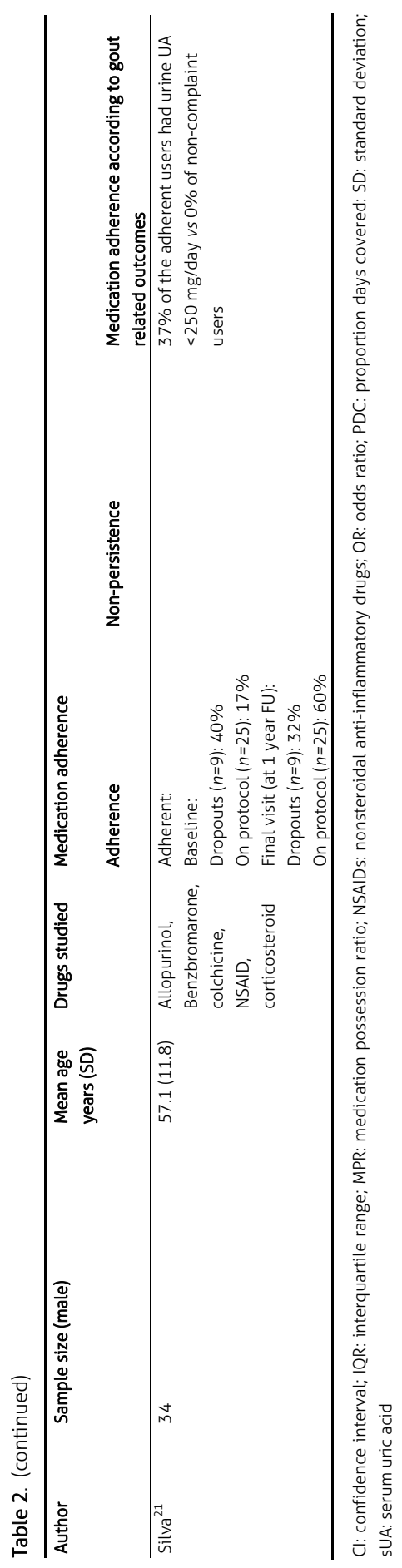


Table 3. Determinants associated with better medication persistence or adherence

\begin{tabular}{|c|c|c|c|c|c|}
\hline Category & Specific factor & Positive & Not significant & Negative & $\begin{array}{l}\text { Level of } \\
\text { evidence }\end{array}$ \\
\hline \multirow[t]{6}{*}{$\begin{array}{l}\text { Social - } \\
\text { economic }\end{array}$} & Age (older) & $\begin{array}{l}\text { Zandman }^{24 c} \\
\text { Solomon }^{27 c} \\
\text { Riedel }^{26 c} \\
{\text { Harrold }(A)^{40 c}}{ }^{25 a} \\
\text { Briesacher }^{25} \\
\text { Silva }^{21} \text { a a }^{31 \text { a }} \\
{\text { Harrold }(B)^{31}} \text { Martini }^{20 a} \\
\text { Lee }^{17 a} \\
\text { McGowan } \\
\text { Rashid }(A)^{28 c}\end{array}$ & $\begin{array}{l}\text { Mantarro } \\
\text { Singh }^{23 c}\end{array}$ & Horsburgh $^{12 b}$ & $\begin{array}{l}\text { Strong, } \\
\text { positive }\end{array}$ \\
\hline & Gender (male) & $\begin{array}{l}\text { Zandman } 24 c \\
\text { McGowan }\end{array}$ & $\begin{array}{l}\text { Solomon }^{27 c} \\
\text { Briesacher }{ }^{25 a} \\
\text { Mantarro } 13 c \\
\text { Singh }^{23 c} \\
\text { Horsburgh } \\
\text { Harrold }(A)^{12 b c} \\
\text { Rashid }(A)^{28 c}\end{array}$ & Riedel $^{26 c}$ & Conflicting \\
\hline & $\begin{array}{l}\text { Race } \\
\text { - African -American / minority race } \\
\text { vs Caucasian } \\
\text { - Mãori vs non-Mãori }\end{array}$ & & & $\begin{array}{l}\text { Solomon }{ }^{27 c} \\
\text { Rashid }(A)^{28 c} \\
\text { Horsburgh }^{12 b}\end{array}$ & $\begin{array}{l}\text { Moderate, } \\
\text { negative }\end{array}$ \\
\hline & Socio-economic status (low vs high) & & Horsburgh $^{12 b}$ & Zandman $24 c$ & Conflicting \\
\hline & Marital status (yes vs no) & Zandman ${ }^{23 c}$ & $\operatorname{Lee}^{17 \text { a }}$ & & Conflicting \\
\hline & Income (low vs high) & & $\operatorname{Lee}^{1 / \mathrm{a}}$ & & $\begin{array}{l}\text { Limited, } \\
\text { no association }\end{array}$ \\
\hline \multirow{6}{*}{$\begin{array}{l}\text { Condition - } \\
\text { related }\end{array}$} & Diagnosis (newly vs previously) & & $\operatorname{Lee}^{17 \text { a }}$ & Sarawate $30 \mathrm{c}$ & Conflicting \\
\hline & Gout flares (more vs less) & $\begin{array}{l}\text { Mantarro }{ }^{13 c} \\
\text { Sarawate } \\
30 c\end{array}$ & & $\begin{array}{l}\text { Rashid }(B)^{c} \\
\text { Silva }^{21 a}\end{array}$ & Conflicting \\
\hline & $\begin{array}{l}\text { Tophaceous gout } \\
\text { Hospitalization (more vs less) }\end{array}$ & Solomon $276 c$ & $\begin{array}{l}\text { Lee }^{17 a} \\
\text { Harrold }(A)^{40 c} \\
\text { Mantarro }{ }^{13 c}\end{array}$ & Solomon $27 c$ & $\begin{array}{l}\text { Conflicting } \\
\text { Conflicting }\end{array}$ \\
\hline & $\begin{array}{l}\text { Health care utilization (more vs } \\
\text { less) }\end{array}$ & Harrold $(A)^{40 c}$ & & & $\begin{array}{l}\text { Limited, } \\
\text { positive }\end{array}$ \\
\hline & Alcohol consumption (yes vs no) & & $\operatorname{Lee}^{17 a}$ & & $\begin{array}{l}\text { Limited, } \\
\text { no association }\end{array}$ \\
\hline & Comorbidity (more vs less) & 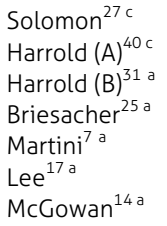 & & & $\begin{array}{l}\text { Strong, } \\
\text { positive }\end{array}$ \\
\hline \multirow[t]{5}{*}{$\begin{array}{l}\text { Comorbiditie } \\
\text { s (yes vs no) }\end{array}$} & Alcohol-related diseases & & Mantarro $^{13 c}$ & & $\begin{array}{l}\text { Limited, } \\
\text { no association }\end{array}$ \\
\hline & Anaemia & & Mantarro ${ }^{13 c}$ & & $\begin{array}{l}\text { Limited, } \\
\text { no association }\end{array}$ \\
\hline & Arrhythmias & & Mantarro $^{13 c}$ & & $\begin{array}{l}\text { Limited, } \\
\text { no association }\end{array}$ \\
\hline & Cancer history & & Zandman ${ }^{24 c}$ & & $\begin{array}{l}\text { Limited, } \\
\text { no association }\end{array}$ \\
\hline & $\begin{array}{l}\text { Cardiovascular disease } \\
\text { Chronic kidney disease }\end{array}$ & $\begin{array}{l}\text { Zandman } \\
\text { Lee }^{17 a} \text { a }\end{array}$ & $\operatorname{Lee}^{17 \mathrm{a}}$ & Rashid $(A)^{28 c}$ & $\begin{array}{l}\text { Conflicting } \\
\text { Conflicting }\end{array}$ \\
\hline
\end{tabular}


Table 3. (continued)

\begin{tabular}{|c|c|c|c|c|c|}
\hline Category & Specific factor & Positive & Not significant & Negative & $\begin{array}{l}\text { Level of } \\
\text { evidence }\end{array}$ \\
\hline & Diabetes & $\begin{array}{l}\text { Zandman }{ }^{24 c} \\
\text { Riedel }^{26 c} \\
\text { Rashid }(A)^{28 c} \\
\text { Mantarro }\end{array}$ & $\operatorname{Lee}^{17 \mathrm{a}}$ & & $\begin{array}{l}\text { Strong, } \\
\text { positive }\end{array}$ \\
\hline & Heart failure & Rashid $(A)^{28 c}$ & Mantarro ${ }^{13 c}$ & & Conflicting \\
\hline & Hypertension & $\begin{array}{l}\text { Zandman } \\
\text { Riedel }^{26 c} \\
\text { Sarawate }^{30 c} \\
\text { Lee }^{17 a} \\
\text { Mantarro } \\
\text { Rashid }(A)^{13 c}\end{array}$ & & & $\begin{array}{l}\text { Strong, } \\
\text { positive }\end{array}$ \\
\hline & Dyslipidaemia & & $\begin{array}{l}\text { Lee }^{17 a} \\
\text { Mantarro } \\
13 c\end{array}$ & & $\begin{array}{l}\text { Limited, } \\
\text { no association }\end{array}$ \\
\hline & Myocardial infarction & Rashid $(A)^{28 c}$ & & & $\begin{array}{l}\text { Limited, } \\
\text { positive }\end{array}$ \\
\hline & Nephrolithiasis & & $\operatorname{Lee}^{17 a}$ & & $\begin{array}{l}\text { Limited, } \\
\text { no association }\end{array}$ \\
\hline & Osteoarthritis & Zandman $^{24 c}$ & Mantarro ${ }^{13 c}$ & & Conflicting \\
\hline & Rheumatoid arthritis & & $\begin{array}{l}\text { Mantarro }{ }^{13 c} \\
\text { Zandman }^{24 c}\end{array}$ & & $\begin{array}{l}\text { Limited, } \\
\text { no association }\end{array}$ \\
\hline & Use of thiazide / other diuretics & Rashid $(A)^{28 c}$ & Mantarro ${ }^{13}$ & & Conflicting \\
\hline \multirow[t]{5}{*}{$\begin{array}{l}\text { Therapy - } \\
\text { related }\end{array}$} & $\begin{array}{l}\text { ULT type (febuxostat vs } \\
\text { allopurinol) }\end{array}$ & McGowan 14 a & $\operatorname{Lee}^{17 a}$ & & Conflicting \\
\hline & $\begin{array}{l}\text { Drug prescription (specialist vs } \\
\text { generalist) }\end{array}$ & $\begin{array}{l}\text { Solomon } 27 c \\
\text { Rashid }(A)^{28 c}\end{array}$ & & & $\begin{array}{l}\text { Limited, } \\
\text { positive }\end{array}$ \\
\hline & Drug doses (higher vs lower) & McGowan ${ }^{14 a}$ & $\operatorname{Lee}^{17 a}$ & $\begin{array}{l}\text { Riedel }^{26 c} \\
\text { Rashid }(A)^{28 c}\end{array}$ & Conflicting \\
\hline & Side effects (yes vs no) & & De Klerk ${ }^{16}$ a & & $\begin{array}{l}\text { Limited, } \\
\text { no association }\end{array}$ \\
\hline & Use of prophylactic (yes vs no) & & $\begin{array}{l}\text { Mantarro }{ }^{13 c} \\
\text { Lee }^{17 a}\end{array}$ & Harrold $(A)^{40 c}$ & Conflicting \\
\hline \multirow[t]{9}{*}{$\begin{array}{l}\text { Patient - } \\
\text { related }\end{array}$} & $\begin{array}{l}\text { Functional capacity, HAQ (greater } \\
\text { vs poorer) }\end{array}$ & & De Klerk $^{16}$ a & & $\begin{array}{l}\text { Limited, } \\
\text { no association }\end{array}$ \\
\hline & $\begin{array}{l}\text { Overall health profile (greater vs } \\
\text { poorer) }\end{array}$ & & De Klerk $^{16 \text { a }}$ & & $\begin{array}{l}\text { Limited, } \\
\text { no association }\end{array}$ \\
\hline & Coping, UCL score (more vs less) & & De Klerk ${ }^{16}$ a & & $\begin{array}{l}\text { Limited, } \\
\text { no association }\end{array}$ \\
\hline & $\begin{array}{l}\text { Perceived health status (high vs } \\
\text { low) }\end{array}$ & & & De Klerk ${ }^{16}$ a & $\begin{array}{l}\text { Limited, } \\
\text { negative }\end{array}$ \\
\hline & $\begin{array}{l}\text { Previous history of non-persistence } \\
\text { to ULT (yes vs no) }\end{array}$ & & $\operatorname{Lee}^{17 \text { a }}$ & & $\begin{array}{l}\text { Limited, no } \\
\text { association }\end{array}$ \\
\hline & $\begin{array}{l}\text { Preference for non- } \\
\text { pharmacological treatments } \\
\text { (yes vs no) }\end{array}$ & & Singh $^{23} \mathrm{~b}$ & & $\begin{array}{l}\text { Limited, } \\
\text { no association }\end{array}$ \\
\hline & $\begin{array}{l}\text { Understanding illness (greater vs } \\
\text { poorer) }\end{array}$ & Dalbeth $(B)^{19}$ a & & & $\begin{array}{l}\text { Limited, } \\
\text { positive }\end{array}$ \\
\hline & Body mass index (high vs low) & Zandman ${ }^{24 c}$ & $\begin{array}{l}\text { Lee }^{17 a} \\
\text { Mantarro } \\
13 c\end{array}$ & & Conflicting \\
\hline & Smoking (current vs never) & & $\begin{array}{l}\text { Lee }^{17 \text { a }} \\
\text { Mantarro } \\
\text { 13c }\end{array}$ & Zandman ${ }^{24 c}$ & Conflicting \\
\hline
\end{tabular}

" Positive association indicates better medication adherence; \# Associated with adherence, but not with persistence; ^ There was a trend for younger age; a crude; $b$ sex, age, race and socioeconomic status adjusted (if applicable); c multivariable adjusted analyses. 
For $\operatorname{six}^{24}$ out of seven studies a better adherence was associated with lower serum uric acid concentration. $13,18,19,21,24,28,29$ One other study showed that time until nonpersistence was longer for those patients with a serum uric acid concentration above $6 \mathrm{mg} / \mathrm{dL}$ compared to those who had a serum uric acid of less than $6 \mathrm{mg} / \mathrm{dL} .{ }^{15}$ Finally, one study found that patients with zero gout flares $74 \%$ were considered adherent (PDC $\geq 80 \%$ ) during 12 months follow-up, while patients with $1-2$ or $\geq 3$ gout flares were $39 \%$ and $28 \%$ adherent, respectively. ${ }^{32}$

\section{DISCUSSION}

Our study shows a large variation in medication adherence to ULT among patients with gout. Poor medication adherence was found in studies using prescription/claims data, whereas studies using MEMS/pill count or patient-reported data showed considerable better adherence rates. In a subgroup of studies $(n=13)$ that reported the proportion of patients adherent based on the commonly used threshold of $80 \%$, meta-analysis revealed a pooled adherence of only $46 \%$. The proportion of patients being nonpersistent, examined in five studies using prescription/claims data, was even lower and showed rates ranging from $56 \%{ }^{27}$ to $87 \%{ }^{30}$ Consistent strong evidence was found that elderly patients, the presence of hypertension and/or diabetes, and those with more comorbidities were more prone to be adherent. Finally, poor medication adherence was associated with higher number of gout flares and an elevated serum uric acid.

Our findings are overall in concordance with a previous systematic literature review of 16 studies from De Vera et al. ${ }^{6}$ We expanded upon that review by adding seven new studies, of which six were conducted outside the USA, and by performing a metaanalysis. Similar results were found across studies conducted inside and outside the USA for which pooled estimate were $45 \%$ and $48 \%$ respectively. As expected, studies using MPR to calculate adherence showed a higher estimate of the proportion of patients being adherent than studies using the more conservative PDC, $44 \%$ and $34 \%$, respectively. Remarkably, one community study conducted in New Zealand and using dispensing records (sample size=732) showed a substantial higher adherence rate of $78 \%$ compared to other studies using prescription/claims data. ${ }^{12}$ This study differed from the others as only patients with a minimum of 90 days of dispensed allopurinol and a minimal follow-up of six months were included. However, results did not change substantially after eliminating the restriction for inclusion on the minimum days of dispensed allopurinol. Whether this high rate is due to an actual better patient adherence, different study population or methodology, remains unclear.

Adherence found in studies using MEMS/pill count $(n=2)$ or patient-reported $(n=6)$ was considerably better. It is known that patient-reported approaches highly overestimate adherence, $^{33}$ due to social desirability. To avoid this bias partially, the use of a validated questionnaire is recommended. Two out of six studies, both conducted in New Zealand by Dalbeth et al. ${ }^{18,19}$ used the MARS questionnaire. Although not specifically validated in 
gout, it was developed to assess adherence to long-term medication regimens. The use of MEMS has been considered to be an accurate method for medication adherence, as it observes 'real-time' behaviour. ${ }^{34}$ The monitor itself might modify medication taking behaviour, but these effects are considered small. ${ }^{35}$

The difference in adherence between prescription/claims opposed to MEMS/pill count data or patient-reported data might not be solely explained by the difference in methods to assess medication adherence, but could also reflect a difference in study population. The majority of prescription/claims studies included gout patients with no ULT prescription 12 months prior the start of the study, whereas studies using patientreported data often included patients with previous use of ULT. These patients might have longer disease duration and might have experienced the negative consequences of not being adherent and therefore greater motivation to take their medication as prescribed. This might also apply to the patients treated by a rheumatologist instead of a general practitioner. Two prescription/claims studies showed indeed that patients receiving ULT from a specialist instead of a generalist had better adherence. ${ }^{27,28}$ Unfortunately in the present review, only a few studies were conducted among patients exclusively under care of a general practitioner ${ }^{2,13}$ or rheumatologist ${ }^{16,17,21}$, due to low number of studies and difference in method to assess adherence it was impossible to conclude whether medication adherence differs between patients seen in first and second line.

Non-persistence was assessed in five prescription/claims studies and rates ranged between $56 \%$ and $88 \%{ }^{14,23,27,30,31}$ This broad range is likely to be explained by the differences in permissible gap length and observation time. A drawback of the studies is the absence of information on reasons to stop or interrupt medication, it could even be provider directed. Thus far, only two studies investigated whether patients return to therapy. Harrold et al. found that among the $70 \%$ of patients who had a gap in therapy of at least 60 -days, about 50\% returned to treatment within 8 months, and $75 \%$ within 4 years. ${ }^{31}$ This was in line with the study from Sarawate et al., who showed that $72 \%$ returned to therapy after experiencing a gap. ${ }^{30}$ None of the studies investigated adherence to medication after restarting therapy. This could be valuable, as it can be assumed that in this subgroup the indication to use ULT is likely more pertinent, and the group that was non-adherent due to severe side effects has likely been excluded.

Nine studies related medication adherence to clinical gout related outcomes. ${ }^{13,15,18,19,21,24,28,29,32}$ For six out of seven studies poor medication adherence was associated with lower serum uric acid concentration ${ }^{13,18,19,21,24,28,29}$ and in one study with fewer gout flares. ${ }^{32}$ However, for all studies reverse causality cannot be excluded, i.e. the dispersion of monosodium urate crystals during the initiation phase of ULT increases the risk of having a gout flare, ${ }^{36}$ which may lead to poor medication adherence. Compared to the review from De Vera et al, the current review reports for 12 additional studies the relationship between medication adherence and potential influencing factors. ${ }^{12-14,16,17,19-21,25,28,31,32}$ Findings strengthened insight into the protective role of older age, presence of hypertension and/or diabetes, and higher number of 
comorbidities on medication adherence. In addition, moderate evidence was found for a role of origin in medication adherence. Further confirmation and exploration of reasons for lower adherence among ethnic minorities is needed. Other patient- and therapyrelated factors, such as knowledge and perceptions of the disease and medication, and health care team and system-related factors ${ }^{36}$ have only been investigated in a few small studies. ${ }^{16,17,19,23}$

The current study should be interpreted within the context of its potential limitations. First, all studies assessed medication adherence by using an indirect method; none of them verified if the patients actually consumed their medication. Second, the thresholds to define 'good' and 'poor' adherence were all arbitrary. Most studies used a cut-off value of $80 \%$, whether this is clinically relevant and required for therapeutic benefit is uncertain. ${ }^{38}$ Third, the wide range of measures and definitions of adherence and nonpersistence complicates the interpretation and summation of the results. In the metaanalysis we therefore only included studies who defined adherent as the proportion of patients who took at least $80 \%$ of their medication during observation. To restrict the influence of other factors we performed a stratified meta-analysis on country of investigation and measure of adherence. Notwithstanding, we cannot exclude that other clinical or methodology factors explain the high heterogeneity of the results. A metaregression analysis would have been more accurate but was impossible, since information on potential variables was often lacking and the total number of studies was too low. Fourth, for two studies the identification of gout patients relied on prescription of ULT only. It is unknown whether the patients had gout or used the medication for other purposes, like tumour lysis syndrome. ${ }^{12,14}$

In conclusion, this study points to the need for more attention to medication adherence to ULT among gout patients. Awareness among clinicians is also important in the view of their role in improving medication adherence. ${ }^{39}$ Combining quantitative and qualitative studies to assess medication adherence are needed not only to reveal the dynamics in medication use but also to understand the rationale for interrupting or stopping medication usage. 


\section{REFERENCES}

1. Wijnands JM, Viechtbauer W, Thevissen K, et al. Determinants of the prevalence of gout in the general population: a systematic review and meta-regression. Eur J Epidemiol. 2015; 30(1):19-33.

2. Kuo CF, Grainge MJ, Mallen $C$, et al. Rising burden of gout in the UK but continuing suboptimal management: a nationwide population study. Ann Rheum Dis. 2015; 74(4):661-667.

3. Dalbeth N, Collis ], Gregory K, et al. Tophaceous joint disease strongly predicts hand function in patients with gout. Rheumatology (Oxford). 2007; 46(12):1804-1807.

4. Rothenbacher D, Primatesta P, Ferreira A, et al. Frequency and risk factors of gout flares in a large population-based cohort of incident gout. Rheumatology (Oxford). 2011; 50(5):973-981.

5. Vrijens B, De Geest S, Hughes DA, et al. A new taxonomy for describing and defining adherence to medications. Br J Clin Pharmacol. 2012; 73(5):691-705.

6. De Vera MA, Marcotte G, Rai S, et al. Medication adherence in gout: A systematic review. Arthritis Care Res (Hoboken). 2014.

7. Martin BC, Wiley-Exley EK, Richards S, et al. Contrasting measures of adherence with simple drug use, medication switching, and therapeutic duplication. Ann Pharmacother. 2009; 43(1):3644.

8. Stroup DF, Berlin JA, Morton SC, et al. Metaanalysis of observational studies in epidemiology: a proposal for reporting. Metaanalysis Of Observational Studies in Epidemiology (MOOSE) group. JAMA. 2000; 283(15):2008-2012.

9. Higgins JPT, Thompson SG. Quantifying heterogeneity in a meta-analysis. Stat Med. 2002; 21(11):1539-1558.

10. Higgins JPT, Thompson SG, Deeks J], et al. Measuring inconsistency in meta-analyses. Brit Med J. 2003; 327(7414):557-560.

11. van Tulder M, Furlan A, Bombardier C, et al. Updated method guidelines for systematic reviews in the cochrane collaboration back review group. Spine (Phila Pa 1976) 2003;28:1290-9.

12. Horsburgh S, Norris P, Becket G, et al. Allopurinol use in a New Zealand population: prevalence and adherence. Rheumatol Int. 2014.

13. Mantarro S, Capogrosso-Sansone A, Tuccori M, et al. Allopurinol adherence among patients with gout: an Italian general practice database study. Int J Clin Pract. 2015; 69(7):757-765.

14. McGowan B, Bennett K, Silke C, et al. Adherence and persistence to urate-lowering therapies in the Irish setting. Clin Rheumatol. 2014.

15. Park H, Rascati KL, Prasla K, et al. Evaluation of health care costs and utilization patterns for patients with gout. Clin Ther. 2012; 34(3):640652.

16. de Klerk E, van der Heijde $D$, Landewe $R$, et al Patient compliance, in rheumatoid arthritis, polymyalgia rheumatica, and gout. J Rheumatol. 2003; 30(1):44-54.

17. Lee $\mathrm{S}$, So MW. Adherence with urate-lowering therapies among male patients with gout in a routine clinical setting. Mod Rheumatol. 2016: 16.

18. Dalbeth N, House ME, Horne A, et al. Prescription and dosing of urate-lowering therapy, rather than patient behaviours, are the key modifiable factors associated with targeting serum urate in gout. BMC Musculoskelet Disord. 2012; 13:174.

19. Dalbeth $N$, Petrie KJ, House $M$, et al. Illness perceptions in patients with gout and the relationship with progression of musculoskeletal disability. Arthritis Care Res (Hoboken). 2011; 63(11):1605-1612.

20. Martini N, Bryant L, Te Karu L, et al. Living with gout in New Zealand: an exploratory study into people's knowledge about the disease and its treatment. J Clin Rheumatol. 2012; 18(3):125129.

21. Silva L, Miguel ED, Peiteado D, et al. Compliance in gout patients. Acta Reumatol Port. 2010; 35(5):466-474.

22. van Onna $M$, Hinsenveld $E$, de Vries $H$, et al. Health literacy in patients dealing with gout: a qualitative study. Clin Rheumatol. 2015; 34(9):1599-1603.

23. Singh JA, Shah N, Edwards NL. A cross-sectional internet-based patient survey of the management strategies for gout. BMC Complement Altern Med. 2016; 16:90.

24. Zandman-Goddard G, Amital H, Shamrayevsky N, et al. Rates of adherence and persistence with allopurinol therapy among gout patients in Israel. Rheumatology (Oxford). 2013; 52(6):11261131.

25. Briesacher BA, Andrade SE, Fouayzi $H$, et al. Comparison of drug adherence rates among patients with seven different medical 
conditions. Pharmacotherapy. 2008; 28(4):437443.

26. Riedel AA, Nelson M, Joseph-Ridge $N$, et al. Compliance with allopurinol therapy among managed care enrollees with gout: a retrospective analysis of administrative claims. J Rheumatol. 2004; 31(8): 1575-1581.

27. Solomon DH, Avorn J, Levin R, et al. Uric acid lowering therapy: prescribing patterns in a large cohort of older adults. Ann Rheum Dis. 2008; 67(5):609-613.

28. Rashid N, Coburn BW, Wu YL, et al. Modifiable factors associated with allopurinol adherence and outcomes among patients with gout in an integrated healthcare system. J Rheumatol. 2015; 42(3):504-512.

29. Halpern R, Mody RR, Fuldeore MJ, et al. Impact of noncompliance with urate-lowering drug on serum urate and gout-related healthcare costs: administrative claims analysis. Curr Med Res Opin. 2009; 25(7):1711-1719.

30. Sarawate CA, Brewer KK, Yang W, et al. Gout medication treatment patterns and adherence to standards of care from a managed care perspective. Mayo Clin Proc. 2006; 81(7):925934.

31. Harrold LR, Andrade SE, Briesacher B, et al. The dynamics of chronic gout treatment: medication gaps and return to therapy. Am J Med. 2010; 123(1):54-59.

32. Rashid N, Levy GD, Wu YL, et al. Patient and clinical characteristics associated with gout flares in an integrated healthcare system. Rheumatol Int. 2015; 35(11):1799-1807.

33. Shi L, Liu J, Koleva $Y$, et al. Concordance of adherence measurement using self-reported adherence questionnaires and medication monitoring devices. Pharmacoeconomics. 2010; 28(12): 1097-1107.

34. Sutton S, Kinmonth AL, Hardeman W, et al. Does electronic monitoring influence adherence to medication? Randomized controlled trial of measurement reactivity. Ann Behav Med. 2014; 48(3):293-299.

35. Sutton S, Kinmonth AL, Hardeman W, et al. Does electronic monitoring influence adherence to medication? Randomized controlled trial of measurement reactivity. Ann Behav Med. 2014; 48(3):293-299.

36. Becker MA, MacDonald PA, Hunt BJ, et al. Determinants of the clinical outcomes of gout during the first year of urate-lowering therapy. Nucleosides Nucleotides Nucleic Acids. 2008; 27(6):585-591.

37. Sabaté E. Adherence to long-term therapies: evidence for action. World Health Organization; 2003.

38. Stamp LK, Merriman T, Frampton C, et al. Plasma oxypurinol as a measure of adherence in clinical trials. Ann Rheum Dis. 2017.

39. Abhishek A, Jenkins W, La-Crette J, et al. Longterm persistence and adherence on uratelowering treatment can be maintained in primary care-5-year follow-up of a proof-ofconcept study. Rheumatology. 2017: 266-270.

40. Harrold LR, Andrade SE, Briesacher BA, et al. Adherence with urate-lowering therapies for the treatment of gout. Arthritis Res Ther. 2009; 11(2):R46. 


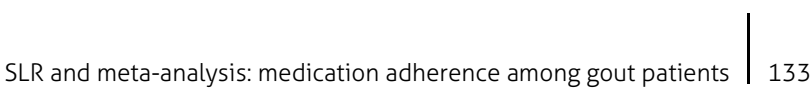

\section{APPENDIX 1. SEARCH STRATEGY}

\section{Pubmed}

(("Gout"[Mesh] OR "Gout" OR "Hyperuricemia"[Mesh] OR "Hyperuricemia" OR "Uric acid"[Mesh] OR "Uric acid" OR "Allopurinol"[Mesh] OR "Allopurinol" OR "Probenecid"[Mesh] OR "Probenecid" OR "Benzbromarone"[Mesh] OR "Benzbromarone" OR "Pegloticase"[Supplementary Concept] OR "Pegloticase" OR "Rasburicase"[Supplementary Concept] OR "Rasburicase") AND ("Patient Compliance"[Mesh] OR "Medication Adherence"[Mesh] OR "Compliance" OR "Adherence" OR "Persistence")) 
134 Chapter 6

\section{APPENDIX 2. SENSITIVITY ANALYSES}

Table S1. Results meta-analysis, proportion of patients adherent

\begin{tabular}{lccccc}
\hline Study & $\begin{array}{c}\text { Sample } \\
\text { size }\end{array}$ & $\begin{array}{c}\text { Proportion } \\
\text { (\%) }\end{array}$ & $\begin{array}{c}\text { 95\% Confidence } \\
\text { interval }\end{array}$ & \multicolumn{2}{c}{ Weight (\%) } \\
Fixed & Random \\
\hline McGowan, Ireland (2016) & 15,908 & 35.3 & 34,6 to 36,1 & 12,60 & 7,89 \\
Rashid (A), USA (2015) & 10,991 & 42.3 & 41.4 to 43.3 & 8.70 & 7.88 \\
Horshburg, New Zeeland (2014) & 732 & 78.0 & 74.8 to 81.0 & 0.58 & 7.62 \\
Kuo, UK (2014) & 49,395 & 39.7 & 39.2 to 40.1 & 39.11 & 7.90 \\
Zandman-Goddard, Israel (2013) & 7,644 & 17.4 & 16.6 to 18.3 & 6.05 & 7.88 \\
Solomon, USA (2008) & 9,823 & 36.3 & 35.3 to 37.2 & 7.78 & 7.88 \\
Halpern, USA (2009) & 10,070 & 43.6 & 42.6 to 44.5 & 7.97 & 7.88 \\
Harrold (A), USA (2009) & 4,166 & 44.0 & 42.5 to 45.5 & 3.30 & 7.85 \\
Briesacher, USA (2008) & 9,175 & 36.8 & 35.8 to 37.8 & 7.27 & 7.88 \\
Sarawate, USA (2006) & 2,405 & 28.0 & 26.2 to 29.8 & 1.90 & 7.81 \\
Riedel, USA (2004) & 5,597 & 56.0 & 54.7 to 57.3 & 4.43 & 7.86 \\
Lee, Korea (2016) & 132 & 71.2 & 62.7 to 78.8 & 0.11 & 6.54 \\
Singh, USA (2016) & 251 & 78.5 & 72.9 to 83.4 & 0.20 & 7.12 \\
Total (fixed effects) & 126,289 & 38.6 & 38.4 to 38.9 & 100.0 & 100.0 \\
Total (random effects) & 126,289 & 46.6 & 40.8 to 51.2 & 100.0 & 100.0 \\
\hline
\end{tabular}

Table S2. Subgroup meta-analysis, proportion of patients adherent to their uric acid lowering therapy

\begin{tabular}{lrrcccc}
\hline Study & $N$ & $\begin{array}{c}\text { Sample } \\
\text { size }\end{array}$ & $\begin{array}{c}\text { Range of } \\
\text { proportions (\%) }\end{array}$ & $\begin{array}{c}\text { Proportion } \\
\text { (\%) }\end{array}$ & $95 \% \mathrm{Cl}$ & $P^{2}$ \\
\hline Overall, random & 13 & 126,289 & 17.4 to 78.5 & 46.0 & 40.8 to 51.2 & $99.7 \%$ \\
Conducted inside USA & 8 & 52,478 & 28.0 to 78.5 & 45.2 & 39.8 to 50.6 & $99.3 \%$ \\
Conducted outside USA & 5 & 73,881 & 17.4 to 78.0 & 47.5 & 36.5 to 58.6 & $99.8 \%$ \\
Outcome measure PDC & 4 & 77,853 & 17.4 to 42.4 & 33.5 & 24.4 to 43.2 & $99.8 \%$ \\
Outcome measure MPR & 6 & 42,456 & 28.0 to 78.0 & 44.1 & 37.6 to 50.7 & $99.4 \%$ \\
\hline
\end{tabular}





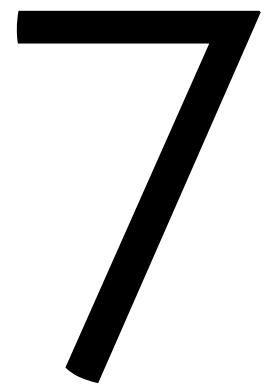

Knowledge, illness perceptions and stated clinical practice behaviour in management of gout: a mixed methods study in general practice 


\section{ABSTRACT}

\section{Objective}

The objective of the present study is to explore knowledge, illness perceptions and stated practice behaviour in relation to gout in primary care.

\section{Methods}

This is a mixed methods study among 32 general practitioners (GPs). The quantitative assessment included the Gout Knowledge Questionnaire (GKO; range 0-10; better) and Brief Illness Perceptions Questionnaire (BIPQ; nine items, range 0-10; stronger). Structured individual interviews obtained further qualitative insight into knowledge and perceptions, in the context of daily practice.

\section{Results}

Among 32 GPs, 18 (56.3 \%) were male, mean age 44.4 years (SD 9.6) and mean working experience 17.1 years (SD 9.7). Median score [interquartile ranges (IQR)] on the GKO was 7.8 [6.7-8.9] and 9.0 [8.0-10.0], when presented as open or multiple-choice questions, respectively. The BIPO (median; [IOR]) revealed that gout was seen as a chronic disease (8.0; [7.0-9.0]), affecting life and emotions moderately (6.5; [5.0-7.0]), having many severe symptoms (8.0; [7.0-9.0]) and in which treatment could be very helpful (8.0; [7.0-9.0]). Further interviews revealed large variation in specific aspects of knowledge and about gaps concerning indications for uric acid-lowering therapy (UALT), duration of UALT, target serum uric acid (sUA) level or duration of prophylactic treatment. Finally, patients' adherence was not checked systematically.

\section{Conclusion}

Specific knowledge gaps and discrepancies between perceptions and stated practice behaviour were identified, which might hamper effective management of this well treatable disease. Improving evidence on the rationale and effectiveness of treatment targets and adherence interventions, tailoring guidelines to general practice and intensification of implementation of guidelines in primary health care seem to be needed. 


\section{INTRODUCTION}

Gout is a chronic rheumatic disease with a reported prevalence of $2.5 \%$ in the UK and $3.9 \%$ in the USA, making it the most common inflammatory joint disease. ${ }^{1-3}$ Despite being a well-treatable disease, it is recognized that the management of gout is suboptimal in both primary ${ }^{4}$ and secondary care. ${ }^{5}$ In a primary care study among patients with gout, low levels of allopurinol prescribing (57\%), serum uric acid (sUA) level testing $(55 \%)$ and achievement of target sUA level ( $<0.36 \mathrm{mmol} / \mathrm{L})(22.4 \%)$ during a 5 -year study period were shown. ${ }^{6}$ In secondary care, adherence to American College of Rheumatology (ACR) guideline recommendations by rheumatologists could be highly improved, shown by a mean adherence score of 5.8 out of 8 ACR guideline recommendations. Low adherence on first-line uric acid-lowering therapy (UALT) dosage, acute prophylaxis dosage and length of prophylaxis was shown. ${ }^{5}$ Common barriers for effective management can be distinguished into patient and physician barriers. Important barriers among patients were not only misperception of the severity and chronicity of gout, but also inadequate patient education resources, resulting in poor adherence to treatment. $^{7-9}$ Among physicians, underestimation of long-term complications and insufficient knowledge about the indications for UALT and about adequate dosing of UALT have already been suggested. ${ }^{10,11}$

On the same line, some qualitative studies explored barriers to effective gout management among patients and physicians. ${ }^{12-16}$ Despite general practitioners (GPs) being the most relevant (in most countries) health care professionals when it comes to diagnosing and treating the disease, the studies in current literature included only a low number of GPs. Therefore, broad insight into how gout is managed by GPs is missing. Notwithstanding, linking knowledge, perceptions and stated practice behaviour is essential when planning to improve gout management for patients with gout.

The current study uses a quantitatively and qualitatively approach to understand knowledge, illness perceptions and stated clinical behaviour of GPs when managing gout with specific attention to the role of UALT, sUA level and prophylactic treatment.

\section{MATERIALS AND METHODS}

\section{Study design and data collection}

A mixed methods approach was used to investigate and understand specific knowledge gaps in pathophysiology and management of gout, illness perceptions about the disease and clinical stated practice behaviours in management. The Good Reporting of A Mixed Methods Study (GRAMMS) guidelines as provided by the Enhancing the OUAlity and Transparency Of health Research (EQUATOR) network were followed. ${ }^{17,18}$

The study was conducted in the southern part of The Netherlands. During a period of 2 weeks, GPs were asked to participate in the study. After agreeing, questionnaires on 
demographics, gout knowledge and gout perceptions were administered, followed by a structured interview to explore in depth understanding and to relate both issues to practice behaviour. The structured interviews were audiotaped and transcribed verbatim. The GPs consented to quote part of the interviews in anonymized form.

\section{Demographics}

General questions about age, sex, years working experience as GP, hours involved in patient care, estimated number of patients with gout per year, practice type, recent education on gout (within the last year, yes/no) and familiarity with gout (on a 0-10 scale, 0 being not familiar and 10 being extremely familiar) were recorded.

\section{Gout knowledge questionnaire}

The Gout Knowledge Questionnaire (GKO) aims to assess knowledge of patients or physicians and addresses ten multiple-choice questions related to the pathogenesis, treatment of acute attacks and also management of chronic gout. ${ }^{19,20}$ GPs were first asked to answer the questions while blind for the answer options. In case that they hesitated (or did not use one of the questionnaire answer options), the interviewer showed the original GKO multiple-choice answers. The GKO was previously translated into Dutch according to the International Society for Pharmacoeconomics and Outcome Research (ISPOR) principles of good practice, ${ }^{21}$ which is consistent with the approach proposed as best practice in rheumatology by Beaton. ${ }^{22}$ As each correct answer provides a score of one, the total score ranges from 0 to 10 , with higher scores indicating better knowledge.

\section{Brief illness perceptions questionnaire}

When completing the validated Dutch version of the Brief Illness Perceptions Questionnaire (BIPO), ${ }^{23,24}$ the GPs were asked to rate their personal perceptions, while imagining that they would suffer from gout with an "average" disease course. The BIPO is a nine-item questionnaire that assesses cognitive and emotional perceptions of a disease, within nine domains (01: consequences, Q2: timeline, Q3: personal control, Q4: treatment control, Q5: identity, Q6: coherence, Q7: emotional representation, Q8: concern, Q9: cause). Q1 to Q8 are scored on an 11-point numeric rating scale (0 to 10), with higher scores representing a more threatening view per domain. Q9 additionally permits to list up to three items that play a causative role in the disease.

\section{Structured interviews}

After completing the questionnaires, the GPs were interviewed to gain more in-depth insight into knowledge and perceptions and link these to clinical practice behaviour, 
specifically with regard to the role of sUA in diagnosis and follow-up, appropriate usage of UALT and the role of adherence in relation to management of the disease.

\section{Data analysis}

Descriptive statistics were used to present the demographics and results of questionnaires, and means with standard deviation (SD) or medians with interquartile ranges [IQR] were used depending on skewness of data.

For qualitative analysis, the verbatim transcripts were read repeatedly and independently by two readers. Using the grounded theory approach, a coding system with categories that were identified in the previous step was developed as well as a taxonomy of the data. ${ }^{25}$ The two readers met regularly to discuss coding and interpretation of data. Wherever necessary, consensus was reached after discussing specific passages or a third reader acted as referee. Representative quotes were collected during data analysis and reported based on the frequency of the particular (and similar) quotes.

\section{RESULTS}

\section{Demographic characteristics}

Thirty-two GPs were interviewed. Eighteen (56.3\%) were male; the mean age was 44.4 years (S.D. 9.6 years); the mean number of years of working experience as GP is 17.1 years (S.D. 9.7 years). The GPs were $34.1 \mathrm{~h}$ (S.D. 11.0) per week involved in patient care, and only four (12.9\%) had followed an educational event on gout in the past year. The estimated number (mean) of new patients with gout in their practice was 8.9 (S.D. 7.0) per year, and familiarity with the disease was scored as 7.0 out of 10.0 (S.D. 1.1) (Table 1).

Table 1. Baseline characteristics for general practitioners (GP) ( $n=32)$

\begin{tabular}{lc}
\hline Age (years), mean \pm S.D. & $44.4 \pm 9.6$ \\
Male sex; $n(\%)$ & $18(56.3)$ \\
Practice type, $n(\%)$ & $15(46.9)$ \\
Group practice & $4(12.5)$ \\
Private practice & $10(31.2)$ \\
Self-employed substitute & $3(9.4)$ \\
Other & $17.1 \pm 9.7$ \\
Years' experience as GP, mean \pm S.D. & $34.1 \pm 10.9[8-55]$ \\
Hours involved in patient care, mean \pm S.D. [range] & $8.9(7.0)[4.3-11.5]$ \\
Estimated new patients with gout per year, mean, (median), [IOQR] & $5(15.6)$ \\
Recent (<1 year) education in gout, $n$ (\%) & $7.0 \pm 1.1[5-9]$ \\
Self-reported Gout familiarity (score 0-10), mean \pm S.D. [range] & \\
Gout Knowledge Questionnaire (score 0-10), mean, (median), [IOQR] & $7.4(7.8)[6.7-8.9]$ \\
Open answers & $9.1(9.0)[8.0-10.0]$ \\
\hline
\end{tabular}




\section{Gout knowledge}

The mean scores (number of correct answers) for the GKO were 7.4 (median 7.8) [IOR 6.7-8.9] and 9.1 (9.0) [8.0-10.0] when answering an open or multiple-choice question with the original answer options, respectively. The numbers (\%) of GPs with correct answers for each item are summarized in Table 2. Questions on the cause of gout (Q1, Q3), signs indicating an acute attack (Q2), treatment of an acute attack (Q4) and recognition of allopurinol being UALT (Q5) were correctly answered by 88 to $100 \%$ of the GPs in the open questioning part, respectively. Questions on flare prevention (09) and comorbidity (010) were answered correctly by 72 and 50\%, respectively, but improved to 97 and 100\% when presenting the original answer options. On the other hand, the question on the target value ("ideal value") (06) was answered correctly by $12 \%$, in the open question, but it increased to $84 \%$ when presenting the original answer options. Finally, the questions about non-pharmacological interventions (Q7) and duration of UALT (Q8) improved to only 75 and $69 \%$ correct answers after seeing the answer options.

\section{Illness perceptions about gout}

The results of the perceptions of GPs about gout are summarized in Table 3. GPS considered gout to be a chronic disease (O2: median 8.0), with (a considerable number of) severe symptoms (05: median 8.0), but with moderate impact on life and emotions (Q1 and Q8: median 6.5), and for which treatment is very helpful (Q4: median 8.0). They believed that gout is not strongly influenced by personal actions (O3: median 4.0). A large variation was observed in perceptions of the amount of concerns gout can raise (O6: median 5.0, IOR 3.3-6.8) and the level of understanding of the disease (median 6.0, IOR 3.3-7.0). Finally, 16 of 32 (50\%) reported diet (alcohol, obesity) to be a major contributing cause of gout (09).

\section{Qualitative analysis on knowledge, beliefs and practice behaviour}

Table 4 shows the most frequent quotes per topic that were collected during the data analysis.

\section{Assessment of serum uric acid and (use) of uric acid lowering therapy}

First, divergent opinions about the usefulness of sUA to diagnose gout were observed. Ten GPs believe that sUA is necessary to diagnose gout, as gout cannot be diagnosed in the absence of hyperuricaemia. Twelve GPS indicated that sUA levels are required in some specific situations, namely the following: (1) "to differentiate gout from other diagnoses in atypical cases" (six GPs) and (2) "to strengthen the diagnosis of gout, which will lead to better treatment" (six GPs). The remaining ten GPs felt that sUA is not necessary and not even useful to diagnose gout. Reasons were (1) "gout is a clinical 
diagnosis, preferably confirmed by joint aspiration" (four GPs) and 2) "a low sUA level does not exclude gout" (three GPS) and "sUA may be low, in particular when patients have an acute gouty arthritis" (three GPs).

Table 2. Gout knowledge level of general practitioners per question ( $n=32)$

\begin{tabular}{|c|c|c|c|}
\hline Question & estion & \multirow{2}{*}{$\begin{array}{c}\text { Open question, } \\
\text { correct answered, } \\
n(\%)\end{array}$} & \multirow{2}{*}{$\begin{array}{l}\text { Multiple-choice, } \\
\text { correct answered, } \\
n(\%) \\
32(100)\end{array}$} \\
\hline 1) & $\begin{array}{l}\text { Q: What causes gout? } \\
\text { Answer options: a) too little calcium, b) too much uric acid, c) an } \\
\text { infection, d) diabetes }\end{array}$ & & \\
\hline 2) & $\begin{array}{l}\text { Q: How do you know if you have an acute attack of gout? } \\
\text { Answer options: a) you have a painful swollen joint, b) you have a } \\
\text { change in blood tests, c) your skin gets red and itchy, d) you have a } \\
\text { lump on your ear }\end{array}$ & $30(93.8)$ & $32(100)$ \\
\hline 3) & $\begin{array}{l}\text { Q: What inside the joint causes attacks of gout? } \\
\text { Answer options: a) bacteria, b) viruses, c) crystals, d) calcium }\end{array}$ & $30(93.8)$ & $32(100)$ \\
\hline 4) & $\begin{array}{l}\text { Q: Which of these is a good treatment during a sudden painful } \\
\text { attack of gout in someone with no other medical condition? } \\
\text { Answer options: a) exercise, b) allopurinol, c) NSAIDs like ibuprofen, } \\
\text { naproxen or indomethacin, d) benzbromarone }\end{array}$ & $32(100)$ & $32(100)$ \\
\hline 5) & $\begin{array}{l}\text { Q: Lowering your blood uric acid can help prevent future attacks of } \\
\text { gout. Which of these drugs can lower your blood uric acid? } \\
\text { Answer options: a) allopurinol, b) prednisone, c) NSAIDs like } \\
\text { ibuprofen, naproxen or indomethacin, d) colchicine }\end{array}$ & $28(87.5)$ & $32(100)$ \\
\hline 6) & Q: What is the ideal blood uric acid level to aim for after treatment & $4(12.5)$ & $27(84.4)$ \\
\hline
\end{tabular}

of gout?

Answer options: a) lower than $0.59 \mathrm{mmol} / \mathrm{L}, \mathrm{b})$ lower than

$0.48 \mathrm{mmol} / \mathrm{L}, \mathrm{c}$ ) lower than $0.36 \mathrm{mmol} / \mathrm{L}, \mathrm{d}$ ) lower than $0.12 \mathrm{mmol} / \mathrm{L}$

7) Q: In order to reduce the serum uric acid, what can you do in addition to medications?

Answer options: a) drink more beer, b) eat more seafood, c) eat more red meat, d) lose weight if you are overweight

8) O: If you are taking a drug to lower your blood uric acid levels, how long do you need to take this drug?

Answer options: a) one month, b) one year, c) two years, d) forever

9) Q: When taking a drug to lower your blood uric acid levels, there can be a temporary increase in gouty attacks. How can you prevent such attacks?

Answer options: a) skip doses of the drug and restart, b) drink less water, c) drink alcohol every day, d) take daily colchicine

10) O: Which is a medical condition that is common in patients with gout?

Answer options: a) high blood pressure, b) cancer, c) AIDS, d) asthma 
Table 3. Results of the BIPO in general practitioners

\begin{tabular}{lcc}
\hline & Mean, (median) [IQR] & $n(\%)$ \\
\hline Q1 Consequences (10 = severely affects life) & $6.2(6.5)[5.0-7.0]$ & $7.5(8.0)[7.0-9.0]$ \\
Q2 Timeline (10 = continues forever) & $4.3(4.0)[3.0-5.0]$ \\
Q3 Personal control (10 = extreme amount) & $7.8(8.0)[7.0-9.0]$ \\
Q4 Treatment control (10 = extremely helpful) & $7.7(8.0)[7.0-9.0]$ \\
Q5 Identity score (10 = many severe symptoms) & $5.0(5.0)[3.3-6.8]$ \\
Q6 Illness concern (10 = extremely concerned) & $5.7(6.0)[3.3-7.0]$ \\
Q7 Coherence (10 = understands very clearly) & $6.2(6.5)[5.0-8.0]$ \\
O8 Emotional representation (10 = extremely affected emotionally) & \\
Q9: Top listed causes: & & $16(50.0)$ \\
1. Diet (alcohol, obesity) & & $13(40.6)$ \\
2. Hereditary & & $12(37.5)$ \\
3. Medication (i.e. diuretics) & \\
\hline
\end{tabular}

Second, reasons to start treatment with UALT were very diverse. The most important reasons were the number of gout attacks per year: "The main reason to start with UALT is when patients have more than 3 gout attacks per year", severity of symptoms: "If patients have fewer attacks (e.g. <3), but the complaints during the attack are severe, then this is a reason to start UALT" and hyperuricaemia in case of a gout attack. Only six GPs mentioned tophi as reason to start with UALT, and three of these GPs determined the effectiveness of UALT, based on the resolution of (if present) tophi.

Third, with regard to duration of UALT, 12 GPS did not prescribe lifelong UALT, for one or more different reasons. Seven of these GPs tried to stop the UALT after 1 year: "If patients have no gout attacks for a longer period of time (e.g. 1 year), I try to reduce and eventually stop UALT"; five GPs suggested that UALT could be stopped after adjustment of lifestyle: "Allopurinol is prescribed lifelong, unless patients change their lives in such a way, you do not expect them to get gout attacks anymore (after weight reduction or stopping diuretics)"; six GPs terminated UALT in the occurrence of renal impairment. One GP thought that allopurinol could be used to treat an acute gouty arthritis.

Fourth, when initiating UALT, nine did not add prophylactic treatment to prevent flares. These GPs advised changing medication/lifestyle (three GPs), prescribed higher doses (or a combination) of UALT in case of flares during the drug start-up phase (three GPs) or waited until the patient was attack-free for a longer period before starting UALT (three GPs). Of the 23 GPs starting colchicine or a non-steroidal anti-inflammatory drug (NSAID) during UALT start-up, none prescribed prophylactic treatment for longer than 2 months: "I combine allopurinol and colchicine to prevent acute gout flares, for a period of 2-4 weeks". (14 GPs).

Finally, to determine effectiveness of UALT, 26 GPs determined sUA, of which six only in case patients continue to have attacks. Seventeen GPs explicitly stated that they did not strive for the target level of $0.36 \mathrm{mmol} / \mathrm{L}$ but based effectiveness of UALT on the absence of new gout attacks and stated that higher sUA levels were acceptable: "The target level of $0.36 \mathrm{mmol} / \mathrm{L}$ is not a strict treatment goal. I accept higher serum uric acid 
levels if the number of acute attacks is decreased". Six GPs never determine sUA to monitor treatment.

Table 4. Themes from qualitative analysis with representative quotes

\begin{tabular}{|c|c|}
\hline Number / Themes & Quotes \\
\hline 1 Knowledge & $\begin{array}{l}\text { "I don't know the target level of serum uric acid; I always look in the lab } \\
\text { form for the reference values." } \\
\text { (which are } 0.20-0.42 \mathrm{mmol} / \mathrm{L} \text { ) }\end{array}$ \\
\hline 2 Illness perceptions & $\begin{array}{l}\text { "Gout is a chronic devastating systemic disease, leading to functional } \\
\text { disability." } \\
\text { "The associated kidney disease or heart failure are very serious } \\
\text { conditions, but the acute attacks are the worst for patients." }\end{array}$ \\
\hline 3 Necessity of uric acid & $\begin{array}{l}\text { "Gout cannot be diagnosed without the presence of hyperuricemia." } \\
\text { "Serum uric acid is not useful, because it will be low in patients with an } \\
\text { acute attack" }\end{array}$ \\
\hline 4 Treatment with UALT & $\begin{array}{l}\text { "The main reason to start with UALT is when patients have more than } 3 \\
\text { gout attacks per year." } \\
\text { "If patients have fewer attacks (e.g. <3), but the complaints are very } \\
\text { severe, then this is a reason to start UALT." }\end{array}$ \\
\hline 5 Duration of treatment with UALT & $\begin{array}{l}\text { "Allopurinol is prescribed lifelong, unless patients change their lives in } \\
\text { such a way, you do not expect them to get gout attacks anymore (after } \\
\text { weight reduction or stopping diuretics)." } \\
\text { "If patients have no gout attacks for a longer period of time (e.g. } 1 \\
\text { year), I try to reduce and thereafter stop the UALT." }\end{array}$ \\
\hline 6 Flare prophylaxis & $\begin{array}{l}\text { "I combine allopurinol and colchicine to prevent acute gout flares, for a } \\
\text { period of } 2-4 \text { weeks." } \\
\text { "I never prescribe allopurinol after an acute flare, first I prescribe } \\
\text { colchicine (or a NSAID) and after } 4 \text { weeks I stop it and start allopurinol." } \\
\text { "I do not prescribe prophylactic treatment, I advise patients to drink } \\
\text { more and sometimes stop diuretics." }\end{array}$ \\
\hline 7 Target level serum uric acid & $\begin{array}{l}\text { "The target level of } 0.36 \mathrm{mmol} / \mathrm{L} \text { is not a strict treatment goal. I accept } \\
\text { higher serum uric acid levels if the number of acute attacks is } \\
\text { decreased." } \\
\text { "If patients have gout, I try to reduce the serum uric acid level below } \\
0.36 \mathrm{mmol} / \mathrm{L} \text { in order to reduce the hyperuricemia-associated risk of } \\
\text { cardiovascular events. Furthermore, I will check and if necessary adjust } \\
\text { cholesterol, blood pressure and glucose." }\end{array}$ \\
\hline 8 Adherence & $\begin{array}{l}\text { "Adherence to UALT is not a problem in patients with gout, since they } \\
\text { are well aware of the fact they will get new gout attacks if they do not } \\
\text { take their medication." } \\
\text { "I think patients with gout take their medication (UALT) very well in the } \\
\text { beginning, but in the course of time become less adherent. Then these } \\
\text { patients will return with a gout flare." } \\
\text { "I have too little time to check whether patients with gout are } \\
\text { adherent." }\end{array}$ \\
\hline 9 Lifestyle advices & $\begin{array}{l}\text { "I refer my patients to a website (www.thuisarts.nl)* where al truths and } \\
\text { untruths about gout are presented. If I am correct, there is no evidence } \\
\text { for all these dietary advices" } \\
\text { "I give the same lifestyle advices as I give patients in cardiovascular } \\
\text { risk management" } \\
\text { "I warn patients for the possible danger of alcohol and organ meats. } \\
\text { Also, I try to motivate them to lose some weight" }\end{array}$ \\
\hline
\end{tabular}

\footnotetext{
* a Dutch website with the most essential information in plain language, understandable by patients, about diseases treated by GPs, an initiative from the Dutch College of GPs.
} 


\section{Adherence to drug therapy}

Nineteen GPs believed that patients with gout are adherent to their drug treatment. "Patients are well aware of the fact new gout attacks will occur if they don't take their medication". Of the 13 GPs that assumed that patients were not adherent to therapy, nine GPs believed that they were adherent in the beginning but stop UALT over time: "I think patients with gout take their medication (UALT) very well in the beginning, but in over the course of time become less adherent". All GPs assumed that these patients would restart therapy themselves in case of a new attack Only eight GPs actively monitored patient adherence by planning appointments at a regular interval (varying from 1 month in the start-up phase to once a year), during which two determined sUA to assess adherence. Seven GPs check adherence when patients had an appointment for any reason. Furthermore, when specifically inquired, 12 confirmed that they checked regularly whether patients pickup their repeat prescriptions, but only electronically and no contact with the nonadherent patients would follow. If non-adherence was recognized (in any way), only 12 GPs would discuss the effects and complications of being non-adherent. Ten GPs admitted to spend insufficient effort in the follow-up of adherence. Main reasons are lack of time or beliefs that patients are adherent anyhow.

\section{Lifestyle advice in patients with gout}

Sixteen GPs believed that diet and drinking habits were main contributing causes of gout (BIPO (Q9)), and all of these mentioned that adjustment of these factors (weight loss, less alcohol, no organ meats, drink more water) would lower sUA in addition to medication. It was therefore surprising to see that only four GPs gave any lifestyle advice(s) to patients with gout. Seven GPs explicitly mentioned that adjustment of diet was outdated.

\section{DISCUSSION}

Our study adds fuel to the ongoing debate about why gout, a treatable disease, is often insufficiently controlled. ${ }^{8,26}$ The strength of this study is that it is the first to address, at the same time, knowledge, illness perceptions and stated clinical practice behaviour in GPs, the medical professionals that commonly diagnose and treat gout. Moreover, the use of a mixed quantitative and qualitative approach allowed to gain in-depth insight into the consequences of gout knowledge and (inadequate) perceptions on gout in general practice, while, at the same time, providing an overall quantification. In Figure 1, we summarized several potential barriers identified in our study and illustrated graphically how these barriers might eventually effect quality of care in patients with gout. 


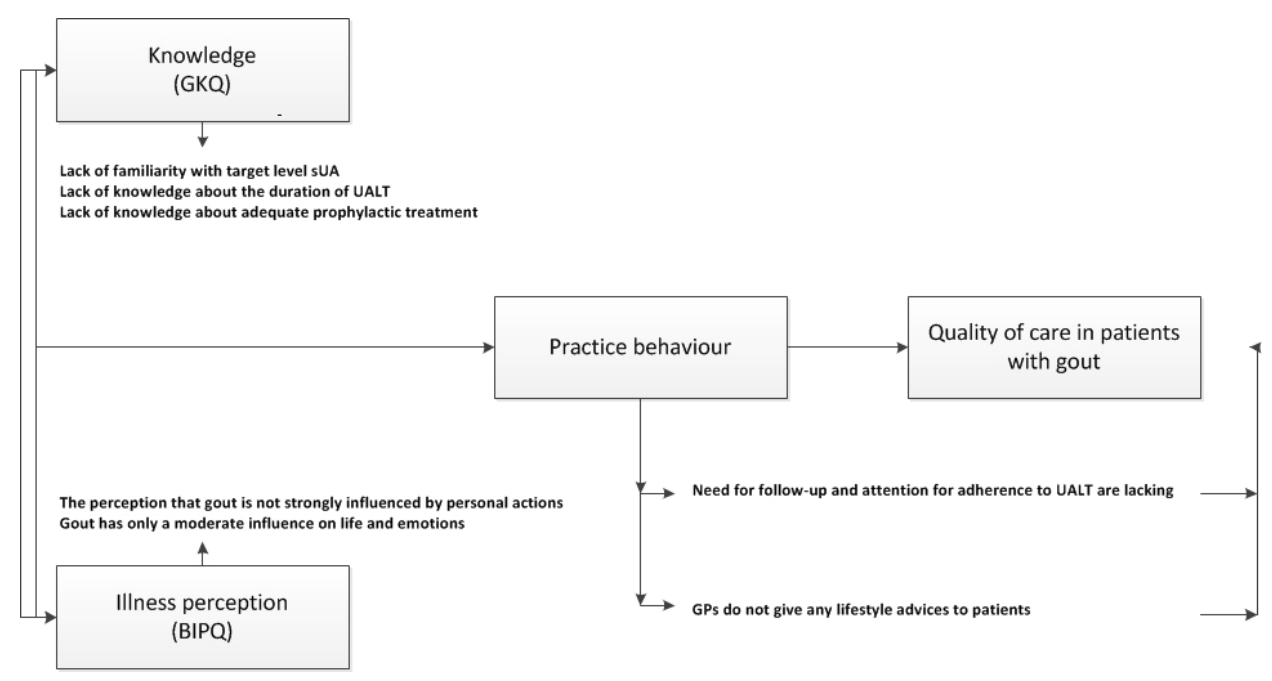

Figure 1. Identified barriers to optimal management in patients with gout treated by general practitioners

The GPs' knowledge, as measured with the GKQ, on pathophysiology, signs and symptoms and treatment of an acute gout attack was mostly excellent, although only half of them indicated that dietary factors play a causative role in gout. However, GKQ-data combined with interviews on knowledge and practice behaviour learned that there is a large variation in the long-term management of gout, specifically in reasons to start UALT, the duration of UALT prescribing and prophylactic treatment at initiation of UALT. The latter finding is in line with one other study already showing inappropriate use of prophylactic colchicine among $74 \%$ of the patients under care of a primary care physician. ${ }^{27}$ Furthermore, although GPs have a pragmatic and realistic view on the evaluation of effectiveness of UALT, it was interesting that most GPs were not aware of the sUA target level of $0.36 \mathrm{mmol} / \mathrm{L}$ as recommended by guidelines and stated to use (if any) the upper limit of laboratory normal ranges $(0.42 \mathrm{mmol} / \mathrm{L})$. Finally, half of the GPs indicated that dietary factors play a causative role in gout, but only few would give lifestyle advices to improve and eliminate causative factors, although it might be attributed partly due to lack of high-quality evidence for specific dietary interventions (avoidance of alcohol, weight loss). ${ }^{28}$ Moreover, lifestyle interventions could have a role in management of gout-associated comorbidities (e.g. cardiovascular diseases, renal disease).

It is well known that also perception of the burden of disease influences the dedication of professionals to a disease and its management. It was therefore reassuring that the GPs perceived gout as a chronic disease with severe symptoms and important impact, in which treatment is very helpful. The GPs' illness perceptions are in accordance with those of 142 patients with gout, which showed that patients also viewed gout as a 
chronic condition responsive to therapy, but not influenced by personal actions. ${ }^{29}$ Nevertheless, there was a striking unawareness among GPs with respect to need for follow-up and/or attention for adherence, as most GPs sincerely believed that patients were adherent to treatment. Other reasons to not make follow-up appointments or check adherence regularly were lack of time or believe that patients who were non-adherent would present themselves automatically when having a new gout flare, actually referring to the patients' personal responsibility. So, even when adherence was checked by the $G P$, actions to improve inadequate adherence were rare.

We realize that the interpretation of the results of our study might be difficult, since we did not actually evaluated quality of care by auditing GPs' adherence to treatment guidelines or quality indicators (OI). Nevertheless, using the ACR and European League Against Rheumatism (EULAR) guidelines as external standard, ${ }^{30-32}$ we implicitly took a large number of the formulated QI by Mikuls et al. ${ }^{33}$ into account. A first example would be the OI about the role of follow-up of sUA level when prescribing UALT: "IF a gout patient is given a prescription for a xanthine oxidase inhibitor, THEN a serum urate level should be checked at least once during the first 6 months of continued use, BECAUSE periodic serum urate measurements are required for appropriate dose adjustments of xanthine oxidase inhibitors (escalations or reductions)". A second example would be the OI about behavioural modifications: "IF a patient is diagnosed with gout and has either (1) obesity (defined as a body mass index $\geq 28 \mathrm{~kg} / \mathrm{m}^{2}$ ) or (2) frequent alcohol use $(\geq 1$ alcoholic beverage per day), THEN as part of their overall therapy, patients should be advised on the importance of weight loss and/or decreased alcohol use, BECAUSE weight loss and reduction of alcohol intake may be beneficial components of gout therapy". On this line, it is important to realize that the guideline on "arthritis", including recommendations how to diagnose and manage gout of the Dutch College of General Practitioners (NHG), currently does not mention a specific sUA level as a treatment target, does not recommend prophylactic treatment when initiating UALT and does not provide specific advises on behavioural modifications, follow-up or monitoring of adherence for patients with gout. ${ }^{34}$ On the other hand, while the GPs' standard mentioned the presence of tophi as indication to initiate UALT, 26 GPs do not mention tophi as a reason to start UALT. Although guidelines are a good starting point to improve quality of care, it is well known that recommendations do not guarantee ubiquitous agreement or compliance with them. Harrold et al. reported among a random sample of US PCPs (including 444 GPs) that only $9.6 \%$ of the GPs were aware of the guidelines and adhered to recommended treatment for acute, intercritical and tophaceous gout in only 47, 3.4 and $12.5 \%$ of the cases, respectively. ${ }^{35}$ In addition to (non)awareness, physicians (including GPs) are experts with strong opinions whom might not always agree with recommendations in guidelines and might question the evidence.

Although already a large amount of evidence is available and summarized in the 2006 EULAR and 2012 ACR guidelines, it should be recognized that the strength of evidence for several recommendations, such as the role and value using SUA as a target for treatment, still needs improvement. As such, we believe that OI that are part of GP's 
audit might be more effective. Nevertheless, these Ol still require strong evidence and a costly organization for monitoring and auditing.

Last, the differences in views between GP and international guidelines might be explained by the heterogeneity of the disease itself and important differences in disease spectra between primary and secondary care will be present. Undoubtedly, GPs treat the milder cases. Therefore, one of the outstanding issues is to collect high-quality registry data in primary care and identify factors that might predict poor prognosis.

This study has other limitations that need to be addressed. First, GPs were recruited from one region in The Netherlands. This might limit the generalizability of these results to all GPs in (and outside) The Netherlands. Nevertheless, we included a broad spectrum of GP that was also representative for the Dutch situation, with regard to years of working experience, sex distribution and age, as this is necessary for qualitative studies (in The Netherlands, $56 \%$ of the GPs are male with a mean age of 48.8 years and of which $46 \%$ have a fulltime employment). As such, the current study represents to date the largest qualitative study in gout. The number of 32 GPs is acceptable from a quantitative view, as for the qualitative part of the study, the theoretical saturation points of information were reached. Second, the GKO was developed as a multiple-choice questionnaire with some of the multiple-choice answers being too obvious in our opinion. Therefore, the questionnaire was presented first with open-ended items (i.e. hiding the answer options), thereby eliciting quotes and thus supporting the qualitative analyses. Finally, in our study, as in any study with qualitative analyses, it might be possible that the interviewer, the questionnaires (that were completed before the interview) or the semistructured character of the interview itself unintentionally influenced the GPs' answers. In conclusion, among a sizable proportion of GPs, we have identified specific knowledge gaps and discrepancies between illness perceptions and stated clinical practice behaviour of GPs that might imply risks for shortcoming patient management in primary health care. Improvement of knowledge of evidence-based treatment targets, implementing adherence interventions and tailoring up-to-date guidelines to general practice are needed to ultimately improve the care of all patients with gout. 


\section{REFERENCES}

1. Kuo CF, Grainge MJ, Mallen C, et al. Rising burden of gout in the UK but continuing suboptimal management: a nationwide population study. Ann Rheum Dis. 2015; 74:6617.

2. Zhu Y, Pandya BJ, Choi HK. Prevalence of gout and hyperuricaemia in the US general population: the National Health and Nutrition Examination Survey 2007-2008. Arthritis Rheum. 2011; 63:3136-41.

3. Wijnands JM, Viechtbauer $W$, Thevissen $K$, et al. Determinants of the prevalence of gout in the general population: a systematic review and meta-regression. Eur J Epidemiol. 2015; 30:1933.

4. Jeyaruban A, Larkins S, Soden M. Management of gout in general practice - a systematic review. Clin Rheumatol. 2015; 34:9-16.

5. Oderda GM, Shiozawa A, Walsh $M$, et al. Physician adherence to ACR gout treatment guidelines: perception versus practice. Postgrad Med. 2014; 126:257-67.

6. Robinson PC, Taylor W], Dalbeth N. An observational study of gout prevalence and quality of care in a national Australian general practice population. J Rheumatol. 2015; 42: 1702-7.

7. De Vera MA, Marcotte G, Rai S, et al. Medication adherence in gout: a systematic review. Arthritis Care Res (Hoboken). 2014; 66:1551-9.

8. Doherty $M$, Jansen $T L$, Nuki $G$, et al. Gout: why is this curable disease so seldom cured? Ann Rheum Dis. 2012; 71:1765-70.

9. Johnston ME, Treharne GJ, Chapman PT, et al. Patient information about gout: an international review of existing educational resources. J Rheumatol. 2015; 42:975-8.

10. Stamp LK, Merriman TR, Barclay ML, et al. Impaired response or insufficient dosage? Examining the potential causes of "inadequate response" to allopurinol in the treatment of gout. Semin Arthritis Rheum. 2014; 44:170-4.

11. Ogdie AR, Hoch S, Dunham J, et al. A roadmap for education to improve the quality of care in gout. Curr Opin Rheumatol. 2010; 22:173-80.

12. Singh JA. Challenges faced by patients in gout treatment: a qualitative study. J Clin Rheumatol. 2014; 20:172-4.

13. van Onna $M$, Hinsenveld $E$, de Vries $H$, et al. Health literacy in patients dealing with gout: a qualitative study. Clin Rheumatol. 2015; 34:1599-603.
14. Harrold LR, Mazor KM, Velten S, et al. Patients and providers view gout differently: a qualitative study. Chronic Illn. 2010; 6:263-71.

15. Spencer K, Carr A, Doherty M. Patient and provider barriers to effective management of gout in general practice: a qualitative study. Ann Rheum Dis. 2012; 71:1490-5.

16. Chandratre P, Mallen CD, Roddy E, et al. "You want to get on with the rest of your life": a qualitative study of health-related quality of life in gout. Clin Rheumatol. 2016; 35:1197-205.

17. O'Cathain A, Murphy E, Nicholl J. The quality of mixed methods studies in health services research. J Health Serv Res Policy. 2008; 13:92-8.

18. Equator Network. The Enhancing the OUAlity and Transparency of health Research (EQUATOR) network website. Available from URL: http://www.equator-network.org.

19. Zhang LY, Schumacher HR, Su HH, et al. Development and evaluation of a survey of gout patients concerning their knowledge about gout. J Clin Rheumatol. 2011; 17:242-8.

20. Li OH, Dai L, Li ZX, et al. Questionnaire survey evaluating diseaserelated knowledge for 149 primary gout patients and 184 doctors in South China. Clin Rheumatol. 2013; 32:1633-40.

21. Wild D, Grove A, Martin M, et al. Principles of good practice for the translation and cultural adaptation process for Patient-Reported Outcomes (PRO) measures: report of the ISPOR Task Force for translation and cultural adaptation. Value Health. 2005; 8:94-104.

22. Beaton DE, Bombardier C, Guillemin F, et al. Guidelines for the process of cross-cultural adaptation of selfreport measures. Spine (Phila Pa 1976). 2000; 25:3186-91.

23. Broadbent E, Petrie KJ, Main J, et al. The brief illness perception questionnaire. ] Psychosom Res. 2006; 60:631-7.

24. de Raaij EJ, Schroder C, Maissan FJ, et al. Crosscultural adaptation and measurement properties of the Brief Illness Perception QuestionnaireDutch Language Version. Man Ther. 2012; 17:330-5.

25. Pope C, Mays N. Qualitative research in health care, 3rd edn. Malden, Mass.: Blackwell Pub./BM] Books 2006.

26. Rashid N, Coburn BW, Wu YL, et al. Modifiable factors associated with allopurinol adherence and outcomes among patients with gout in an integrated healthcare system. J Rheumatol. 2015; 42:504-12. 
27. George M, Pullman-Mooar S, Hussain F, et al. Evaluating appropriate use of prophylactic colchicine for gout flare prevention. Arthritis Care Res (Hoboken). 2012;66:1258-62.

28. Moi JH, Sriranganathan MK, Falzon L, et al. Lifestyle interventions for the treatment of gout: a summary of 2 Cochrane systematic reviews. J Rheumatol. 2014; Suppl 92:26-32.

29. Dalbeth N, Petrie KJ, House M, et al. Illness perceptions in patients with gout and the relationship with progression of musculoskeletal disability. Arthritis Care Res (Hoboken). 2011; 63:1605-12.

30. Zhang $W$, Doherty $M$, Bardin $T$, et al. EULAR evidence based recommendations for gout. Part II: management. Report of a task force of the EULAR Standing Committee for International Clinical Studies Including Therapeutics (ESCISIT). Ann Rheum Dis. 2006; 65:1312-24.

31. Khanna D, Fitzgerald JD, Khanna PP, et al. 2012 American College of Rheumatology guidelines for management of gout. Part 1: systematic nonpharmacologic and pharmacologic therapeutic approaches to hyperuricaemia. Arthritis Care Res (Hoboken). 2012; 64:1431-46.

32. Khanna D, Khanna PP, Fitzgerald JD, et al. 2012 American College of Rheumatology guidelines for management of gout. Part 2: therapy and antiinflammatory prophylaxis of acute gouty arthritis. Arthritis Care Res (Hoboken). 2012; 64:1447-61.

33. Mikuls TR, MacLean CH, Olivieri J, et al. Quality of care indicators for gout management. Arthritis Rheum. 2004; 50:937-43.

34. Janssens $\mathrm{HJ} L \mathrm{LH}$, Van Peet PG, Gorter KJ, et al. NHG Standaard Artritis. Huisarts Wet. 2009; 52:439-53.

35. Harrold LR, Mazor KM, Negron A, et al. Primary care providers' knowledge, beliefs and treatment practices for gout: results of a physician questionnaire. Rheumatology (Oxford). 2013; 52:1623-9 



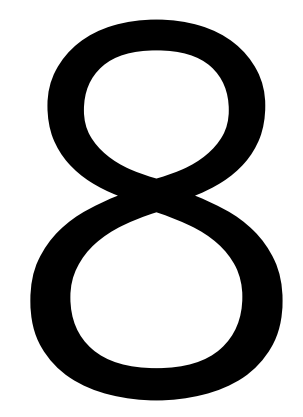

Summary and general discussion 

\begin{tabular}{l|l} 
& \\
Summary and general discussion & 155
\end{tabular}

\section{SUMMARY AND GENERAL DISCUSSION}

Gout and its main risk factor hyperuricemia have been associated with several comorbidities, including hypertension. However, the underlying mechanism remains uncertain. Considerable research efforts have been made to understand the role of uric acid as an independent risk factor for the development of hypertension. Thus far, studies focused mostly on the role of total serum uric acid and have ignored the distinction between uric acid concentration and its production. Since there biological evidence that the production of uric acid may contribute, independent of uric acid concentration, to the pathogenesis of hypertension, the production should be investigated as well. ${ }^{1,2}$ In Part I of the thesis we therefore investigated the role of uric acid production in the association between uric acid and blood pressure.

Gout is a well-manageable disease. In patients with recurrent gout flares or tophi, it is recommended to start long-term uric acid lowering therapy to reduce the number of gout flares and resolve tophi. ${ }^{3}$ Notwithstanding, a substantial proportion of patients with gout continue to experience multiple gout flares, signifying insufficient control of the disease. ${ }^{4}$ To improve the management of gout, studies exploring the barriers for effective gout management among both patients and physicians are needed. ${ }^{5}$ In Part II of this thesis we explored first medication adherence in patients with gout requiring uric acid lowering therapy and next the knowledge, illness perceptions and stated clinical practice behaviour of general practitioners when managing gout.

This chapter summarizes the main findings of the studies in this thesis and discusses the most important methodological considerations. Finally, the clinical implications are presented and some directions for future research are proposed.

\section{MAIN FINDINGS}

\section{Part I Uric acid and blood pressure: The role of uric acid production}

In each of the Chapters 2-4, we tested the hypothesis that the production of uric acid is associated with blood pressure and hypertension. Three different proxies for uric acid production were investigated.

In Chapter 2, genetic variation in xanthine oxidoreductase (XOR) as a proxy for uric acid production was used to investigate whether uric acid production was associated with secular trends in mean arterial pressure, pulse pressure and the development of hypertension. Longitudinal data (median follow-up of 8.8 years) from 2769 participants of the Flemish Study on Environment, Genes, and Health Outcomes (FLEMENGHO) and European Project on Genes in Hypertension (EPOGH) study was used. Three of 25 tagging XOR single nucleotide polymorphisms (SNPs) were associated with the increase in pulse pressure (rs11904439), or mean arterial pressure (rs2043013), or the risk of 
hypertension ( $r$ 148756340 and rs11904439). In normotensive participants ( $n=2050$ ) the risk of hypertension was 30-70\% higher in minor allele carriers of rs 148756340 and rs11904439, respectively. With a false discovery rate set at 0.25 , the aforementioned associations retained significance. Overall, these findings suggest that variation in uric acid production might be associated with secular trends in the steady and pulsatile blood pressure components and the risk of hypertension. It is of note, serum levels of uric acid at baseline ( $n=1949)$ were not associated with variation in XOR ( $P$-value $\geq 0.05)$. Overall, these findings suggest that variation in uric acid production might be associated with secular trends in the steady and pulsatile blood pressure components and the risk of hypertension.

In Chapter 3, ratios of the different purine metabolites (precursors of uric acid) as proxies for XOR activity were used to examine the association between uric acid production and blood pressure in school-age children. An additional aim was to extend existing evidence on the relation between plasma uric acid concentration and blood pressure to this young population. Cross-sectional data from 246 children from the KOALA Birth Cohort Study was used. Higher ratios of uric acid/xanthine and xanthine/hypoxanthine, indicating higher XOR activity, were associated with higher diastolic blood pressure. Furthermore, higher plasma uric acid concentration was associated with higher diastolic blood pressure. However, no association with systolic blood pressure was found. Overall, these findings suggest that uric acid production was associated with blood pressure. In addition, plasma uric acid concentration was significantly associated with blood pressure already in school-age children.

Finally, in Chapter 4 24-h uric acid excretion in urine as a proxy for uric acid production was used to investigate whether uric acid production was associated with the steady and pulsatile blood pressure components and the prevalence of hypertension. In addition, we investigated whether serum uric acid was associated with one of these outcomes. Cross-sectional data from 2555 participants of The Maastricht Study was used. After adjustment for traditional hypertension risk factors, serum uric acid and 24-h urinary uric acid excretion were both associated with mean arterial pressure and hypertension. The association of both serum and urinary uric acid with mean arterial pressure remained significant after further adjustment for urinary or serum uric acid, respectively. These findings suggest that both serum uric acid and its production were, independent of each other, associated with mean arterial pressure and hypertension.

Taken together, the findings from Chapters 2-4 suggest that uric acid production might be associated with elevated blood pressure, in particular with the steady component of blood pressure and hypertension. Regardless of the difference in studied proxies and study populations, our results point towards the same direction. 


\section{Part II Gout management by the patient and general practitioner}

In Chapter 5, medication adherence among gout patients was investigated. A retrospective cohort study was conducted using the UK Clinical Practice Research Datalink (CPRD). Among 48,280 newly diagnosed gout patients initiated allopurinol the vast majority had poor medication adherence. Only $38 \%$ of the patients were considered adherent, defined as the proportion of days covered (PDC) of at least $80 \%$ during the entire observation time (mean of 5.5 years). Non-persistence, defined as the occurrence of a first gap of at least 90 days, increased from $39 \%$ to $57 \%$ after 1 and 5 years of initiation, respectively. After the occurrence of a first medication gap, $43 \%$ restarted therapy within 1 year, yet 52\% experienced again a 90-day gap in the first year after re-initiation. In particular females and current smokers were at increased risk for non-persistence and non-adherence, while those being overweight, taking antihypertensive medication, and suffering from comorbidities such as dementia, diabetes, dyslipidaemia, and depression were more likely to be persistent and adherent.

In Chapter 6, we conducted a systematic literature review on medication adherence among gout patients treated with uric acid lowering therapy. Of the 23 included studies, medication adherence was much higher when assessed by electronic monitoring device $(n=1)$, pill count $(n=1)$, or patient-reported $(n=6)$ data than when assessed by prescription/claims data $(n=15)$. Of the 23 studies considered, 13 met the criteria of defining adherence at a threshold of $80 \%$ and were included in the meta-analysis. The pooled proportion of adherent patients among those studies was $46 \%$, with very high heterogeneity $\left(I^{2}>99 \%\right)$. Subgroup analyses were performed to determine if this heterogeneity could be explained by the country of investigation or measure of adherence. The pooled proportion of patients adherent was similar across studies conducted in the USA $(n=8 ; 45 \%)$ and other countries $(n=5 ; 48 \%)$. The pooled proportion of patients adherent was higher in studies using medication possession ratio (MPR) ( $n=6 ; 44 \%)$ compared to studies using proportion days covered (PDC) ( $n=4 ; 34 \%)$ to measure adherence. Non-persistence, occurrence of an interruption in therapy, was reported in five studies, but the heterogeneity in defining non-persistence $(30-, 60-$ or $90-$ day interruption) made comparisons difficult. When considering the five studies and all possible gap lengths, more than $50 \%$ of the patients became non-persistent in the first year after starting uric acid lowering therapy. Poor medication adherence was associated with elevated serum uric acid as well as frequent gout flares. Factors associated with better medication adherence were older age, higher number of comorbidities, and the presence of diabetes or hypertension.

In Chapter 7, we explored the knowledge, illness perception, and stated practice behaviour in relation to gout among 32 general practitioners. By using a mixed quantitative and qualitative approach, we showed that knowledge on pathophysiology, signs and symptoms, and treatment of an acute gout attack was mostly excellent among general practitioners. However, there was a large variation in the stated long-term 
management of gout, specifically concerning lifestyle advice and the use of uric acid lowering therapy. General practitioners perceived gout as a chronic disease with severe symptoms and important impact, in which treatment is very helpful. Nonetheless, there was a striking unawareness with respect to need for follow-up visits and/or attention for medication adherence.

Overall, from Chapters 5-7 we can conclude that gout management was far from optimal. We showed poor medication adherence, in particular among younger gout patients, those with less comorbidities and not using other medications on a regular basis. Moreover, among general practitioners knowledge and illness perceptions of gout and its acute treatment were adequate and mostly excellent, whereas long-term management, including accurate prescription of uric acid lowering therapy, lifestyle advice, and adherence support were insufficient.

\section{URIC ACID PRODUCTION AND BLOOD PRESSURE}

While the strengths and weaknesses of the individual studies were described in the discussions of the respective chapters, it is worthwhile to add general and overarching reflections on (i) the challenges to measure uric acid production, (ii) influence of uric acid production on the different blood pressure components and (iii) and directions for future research.

\section{Challenges to measure uric acid production}

In the absence of a feasible approach to directly measure the production of uric acid three different proxies were investigated: variation in the $X O R$ gene, ratios of different purine metabolites (hypoxanthine, xanthine, and uric acid), and 24-h urinary uric acid excretion. For each proxy the advantages and disadvantages are summarized in Table 1.

Table 1. The pros and cons for measures explored as proxies for uric acid product in this thesis

\begin{tabular}{|c|c|c|c|c|}
\hline Proxy & $\begin{array}{l}\text { Identification of } \\
\text { overproducers }\end{array}$ & Drawbacks & Reliable & Participants burden \\
\hline $\begin{array}{l}\text { SNPS in the } X O R \\
\text { gene }\end{array}$ & $\begin{array}{l}\text { Partly, only those with } \\
\text { increased XOR activity }\end{array}$ & $\begin{array}{l}\text { - Linkage disequilibrium } \\
\text { - Pleiotropic effect }\end{array}$ & $\begin{array}{l}\text { Yes: the genotype is } \\
\text { constant during life }\end{array}$ & $\begin{array}{l}\text { Low: one sample is } \\
\text { sufficient, e.g. from a } \\
\text { buccal swab }\end{array}$ \\
\hline $\begin{array}{l}\text { Ratios of purine } \\
\text { metabolites }\end{array}$ & $\begin{array}{l}\text { Partly, only those with } \\
\text { increased XOR activity }\end{array}$ & $\begin{array}{l}\text { Feedback mechanisms, } \\
\text { e.g. increased xanthine } \\
\text { excretion via the kidneys }\end{array}$ & $\begin{array}{l}\text { Time dependent: might } \\
\text { be influenced by diet, } \\
\text { physical activity, and } \\
\text { medication use }\end{array}$ & $\begin{array}{l}\text { Moderate: collection of } \\
\text { one or multiple blood } \\
\text { samples }\end{array}$ \\
\hline $\begin{array}{l}\text { Uric acid excretion in } \\
24-h \text { urine }\end{array}$ & $\begin{array}{l}\text { Yes, those with increased } \\
\text { XOR activity AND increased } \\
\text { purine supply }\end{array}$ & $\begin{array}{l}\text { Feedback mechanisms, } \\
\text { e.g. increased uric acid } \\
\text { excretion via the intestine }\end{array}$ & $\begin{array}{l}\text { Time dependent: might } \\
\text { be influenced by diet, } \\
\text { physical activity, and } \\
\text { medication use }\end{array}$ & $\begin{array}{l}\text { Moderate - high: collection } \\
\text { of one or multiple } 24-\mathrm{h} \\
\text { urine collections }\end{array}$ \\
\hline
\end{tabular}


First, variation in the $X O R$ gene was used as a proxy for the enzyme activity of XOR. A genetic variant can function as a proxy for the exposure with the advantage that it reduces the potential for confounding and eliminates reverse causation. ${ }^{6,7}$ This approach, called Mendelian randomization, can be considered as a natural randomized controlled trial., ${ }^{6,7}$ Such an approach is useful in order to test our hypothesis. Furthermore, it is relatively easy and inexpensive to obtain DNA and to genotype the individual. Via a single buccal swab DNA can be extracted, this is inexpensive, not invasive and can easily be obtained at home, with the advantage that this is not timeconsuming and burdensome for the participant. ${ }^{8,9}$

Although it is well established that the XOR gene encodes the enzyme XOR, the functionality of the investigated SNPs is unknown at present. Therefore, we do not know if these SNPs alter enzyme activity and subsequently increase the production of uric acid. In addition, a potential limitation by using genetic variation as a proxy for the exposure is that there might be linkage disequilibrium between variants of one gene to polymorphisms in other genes. Confounding will result if both the variant of interest and that with which it is in linkage disequilibrium are both associated with elevated blood pressure. ${ }^{7}$ Another potential limitation is the chance of a pleiotropic effect. Pleiotropy is the phenomenon in genetics whereby a variant in the gene influences multiple phenotypes. ${ }^{10}$ Interpretation of associations between variants in XOR and blood pressure might not be straightforward if the variant has pleiotropic effects. ${ }^{7}$ It is unlikely that pleiotropy plays a significant role in our study, since $X O R$ mainly catalyses the oxidation of hypoxanthine and xanthine. Another disadvantage, patients with increased purine supply will not be identified, limiting the discovery of overproduction of uric acid to a subgroup of individuals with increased XOR activity.

Second, ratios of the purine metabolites hypoxanthine, xanthine and uric acid were used as a proxy for increased XOR activity. As described in the introduction section, in animal studies and studies examining patients with XOR deficiency a decreased concentration of upstream metabolites was found. ${ }^{11-14}$ We assumed an opposite effect in case of increased XOR activity, so a relatively lower hypoxanthine compared to xanthine and lower xanthine compared to uric acid concentration, but whether this opposite effect actually occurs requires further investigation. It is important to take into account that the activity of XOR also relies on regulatory feedback systems as well as concentrations and activities of other enzymes involved in the pathway. ${ }^{15}$ For example, an accumulation of the purine metabolites might be compensated by alterations in urinary excretion of xanthine or hypoxanthine, ${ }^{16}$ or the degradation of hypoxanthine to inosine monophosphate (IMP) and xanthine to xanthosine monophosphate (XMP) by hypoxanthineguanine phosphoribosyltransferase (HGPRT). ${ }^{14}$ Another disadvantage is that only people with increased XOR activity will be identified.

The advantage of using plasma purine metabolites as proxy is that blood samples are easily obtained, the collection is slightly burdensome for the participants, and the metabolites can even be determined in stored samples. The collection of a blood sample is slightly burdensome for the participant. 
$160 \mid$ Chapter 8

Third, 24-h urinary uric acid excretion was used as a proxy for increased uric acid production. Previous studies showed that an increase in uric acid production, from either endogenous or exogenous sources, increases uric acid excretion. ${ }^{17,18}$ Since uric acid is predominantly excreted by the kidneys, we assumed to find an increase urinary excretion. However, whether the relative contribution of renal and extra-renal excretion is comparable in case of increased uric acid production or disorders in one of the eliminations pathways needs further investigation. ${ }^{19-22}$ An advantage of urinary uric acid excretion as a means to identify uric acid overproducers is that overproducers, either because of increased XOR activity or increased purine intake, can be identified. A disadvantage is the collection of 24-urine samples, which is time-consuming and burdensome for the patient. The collection itself might therefore influence daily life regarding diet and physical activity and influences uric acid production and its subsequent excretion.

With regard to all laboratory measurements of uric acid and purine metabolites, it is of note that they were determined in a single sample. For study purposes the variability in concentrations was minimized by measuring all individuals under the same condition; in the KOALA study children were asked not to eat or drink 1.5 hours prior to and during the home visit, resulting in a mean fast time of 2.2 hours (standard deviation of 1.2 hours). In The Maastricht Study, blood values from the participants were measured after an overnight fast and urinary uric acid was collected over a 24-h time period. Despite the standardized protocol, biological variability due to diet, physical activity, diurnal cycles and seasonal rhythms might have influenced the concentration and cannot be excluded. ${ }^{23-27}$ While repeated measurements will improve reliability, they are timeconsuming and more burdensome for the participant.

From none of the proxies studied we have information on the form of the enzyme responsible for the oxidation. The original form xanthine dehydrogenase (XDH), preferentially reduces nicotinamide adenine dinucleotide $\left(\mathrm{NAD}^{+}\right)$to $\mathrm{NADH}$, whereas xanthine oxidase $(X O)$, particularly present in the circulation, reduces molecular oxygen. Only for the latter form of the enzyme applies that during oxidation the reactive oxygen species (ROS) superoxide and hydrogen peroxide are generated as by products. ${ }^{28-34}$ Because of the presence of two form of the XOR enzyme, the clinical impact of increased uric acid production is likely to depend on this. Several factors can influence and induce the conversion of $\mathrm{XDH}$ to $\mathrm{XO}$, for example hypoxia and ischaemia. ${ }^{35}$ The presence of these conditions might indicate that the oxidation is more often carried out by $X O$ then when there is absence of these conditions.

In conclusion, among the proxies investigated in this thesis 24-h urinary uric acid excretion would be the most feasible and comprehensive proxy to identify uric acid overproducers. In this case, those with increased XOR activity and those with increased uric acid production can be identified. 
\begin{tabular}{l|l} 
& \\
Summary and general discussion & 161
\end{tabular}

\section{Blood pressure as outcome}

A second aspect worthwhile discussing when studying the relation between uric acid and blood pressure is the measurement of blood pressure itself. Different approaches can be liable to different types of measurement errors. In addition, the association of uric acid production with the steady and pulsatile blood pressure component should be discussed, as these might reflect different pathophysiological states.

\section{Measurement of blood pressure}

In the FLEMENGHO and EPOGH study, the average of five conventional blood pressure measurements was used to report blood pressure of individuals. A limitation of conventional blood pressure measurement is that the presence of masked hypertension (normal office blood pressure but ambulatory or home blood pressure readings are in the hypertensive range) or white coat hypertension (elevated office blood pressure but ambulatory or home blood pressure readings are in the normal range) cannot be excluded. A previous study showed that among untreated persons, by using 24-h ambulatory blood pressure measurement as the gold standard, the prevalence was as high as $20 \%$ for masked and $10-15 \%$ for white-coat hypertension. ${ }^{37,38}$ In The Maastricht Study we used 24-h ambulatory blood pressure measurement, in this case patients with masked hypertension will be identified and those patients with white-coat hypertension will not be identified as having hypertension. In the KOALA Birth Cohort Study, the child's blood pressure was measured by a trained nurse and by the parents. In the discussion section of Chapter 3 the possible advantages and possible measurement errors by using nurse and parent measured blood pressure have already been extensively discussed.

\section{Blood pressure components}

As indicated in the introduction, the association of uric acid production with the specific blood pressure components should be discussed as these might reflect different pathophysiological states. The three proxies for uric acid production were associated with the steady blood pressure component and in one study with the pulsatile component, represented by mean arterial pressure or diastolic blood pressure and represented by pulse pressure, respectively (Table 2 ).

Table 2. Summary of the results between the studied proxies and the different blood pressure components

\begin{tabular}{lllll}
\hline & Age of the population & Steady BP & Pulsatile BP & Hypertension \\
\hline Variants in the XOR gene & All ages & MAP & PP & Yes \\
Ratios of purine metabolites & School-age, 7 years & DBP & SBP $(P>0.50)$ & NA \\
Uric acid excretion in 24-h urine & $40-75$ years & MAP & PP $(P=0.18)$ & Yes \\
\hline
\end{tabular}

BP is blood pressure; DBP: diastolic blood pressure; MAP: mean arterial pressure; NA: not applicable; PP: pulse pressure; SBP: systolic blood pressure. Blood pressure component in bold indicate a significant association with the investigated proxy. 
In the KOALA Birth Cohort study among school-aged children we found an association between two ratios of the purine metabolites with diastolic but not with systolic blood pressure (all $P$-values $>0.50$ ). This is in line with the idea that an increase in uric acid production increases vascular resistance but would initially lead only to an elevation in the steady blood pressure component, known as first phase., 28,39-41 After prolonged exposure, this might lead to embedding of the vessel structure and eventually an increase in the pulsatile blood pressure component, considered as the irreversible second phase. ${ }^{42,43}$ Because of the young study population it was not possible to investigate the effects of prolonged exposure. In the older study population of The Maastricht Study, which consisted of individuals between 40-75 years of age, increased urinary uric acid excretion was associated with mean arterial pressure. The association with pulse pressure $(P=0.15)$ was closer to significance compared to the KOALA Birth Cohort Study, but still insignificant.

In the FLEMENGHO and EPOGH study, there was no restriction on age and the study population consisted of individuals from ten years of age and older. Three SNPs of the XOR gene were associated with mean arterial pressure, and we also found an association with pulse pressure, in contrast with the KOALA Birth Cohort Study and The Maastricht Study. Of interest would be to investigate whether elevated pulse pressure results from prolonged exposure to increased uric acid production. Stratification on the duration of increased uric acid production would be a good approach, since polymorphisms are age independent a stratification by age could be used. However, only 159 out of 2050 individuals were minor allele carriers for rs11904439, and stratifying the group according to age was not possible because of insufficient statistical power.

\section{Implications for clinical practice and future directions}

As yet, the findings from our studies on the role of uric acid production in bloodpressure and hypertension have no clinical implications. Several steps are still required before it is possible to identify overproducers of uric acid and to collect evidence that overproduction leads to hypertension and subsequent cardiovascular disease. First, the studied proxies, or other proxies reflecting increased uric acid production, need to be validated. Second, after validation the current findings need to be confirmed. Third, it needs to be established at which thresholds proxies confer a sufficient risk for high blood pressure or hypertension. Finally, evidence needs to be collected whether a reduction in uric acid production, for example by the administration of an $\mathrm{XO}$ inhibitor, reduces the risk of high blood pressure and hypertension.

From a biological point of view, the relation between uric acid overproduction and blood pressure is complex and in this thesis we have taken a simplified view on this. Uric acid production is a process involving various factors that regulate the flux through the purine pathway. It depends besides on the activity of XOR and purine supply on the concentration and activities of all enzymes involved in the pathway, and the activity of feedback and other regulatory control systems. ${ }^{15}$ A system biology approach can be of 
additional value to study the entire flux of uric acid production, and eventually its association with blood pressure.

If future studies are able to convincingly demonstrate a relation between uric acid production and hypertension, it might have potential clinical relevance. Even if the effect is small, given the burden of chronic hypertension and the fact that only $37 \%$ of the patients obtain a well-managed blood pressure, controlling uric acid production might have clinical benefit. ${ }^{44}$ Moreover, identifying overproducers of uric acid may have implications beyond patients with hyperuricemia. Since overproduction can also be a problem in individuals with normal uric acid concentrations. If their excretion capacity is adequate, they will not develop hyperuricemia, but may still suffer from the adverse consequences of increased uric acid production.

\section{GOUT MANAGEMENT}

Chapters in Part // revealed large variation in medication adherence in gout patients and showed a paradox in general practitioner knowledge on the long-term management of gout and their stated clinical behaviour. In addition to considerations raised in the discussion of each chapter, we will further reflect on (i) the methodology used to assess medication adherence, (ii) the reason for poor medication adherence, (iii) the role of the guidelines for optimal gout management, and (iv) implications for future research and further directions.

\section{Assessing medication adherence}

Our analyses in CPRD combined with the systematic literature review revealed large differences in medication adherence in studies using prescription/claims data compared to those using Medication Event Monitoring System (MEMS), pill count, or using patientreported data. Several factors might explain this discrepancy, among which the method of assessing adherence and selection of the study population seem the most important. The advantages and disadvantages of the different methods to assess medication adherence are summarized in Table 3.

Overall, studies based on prescription/claims data $(n=15)$ showed poor adherence to medication, independent of country of investigation and measure used to assess adherence. The main advantage of using prescription/claims data is that large populations can be studied and that any distortion caused by patient recall or desire to give socially accepted answers is eliminated. ${ }^{46}$ A disadvantage, which also applies for our study conducted in CPRD, is that over-the-counter medication use, prescriptions in secondary care, and prescriptions filled are often not available. Subsequently, the number of gout patients considered to have poor medication adherence may therefore be overestimated. To minimize this overestimation in defining non-persistence a gap length of at least 30-days is considered. In this case an interruption of a few days or 
$164 \mid$ Chapter 8

weeks due to hospitalization, emergency supply, or holiday will not automatically lead to misclassification.

Table 3. Methods for measuring medication adherence. ${ }^{45}$

\begin{tabular}{|c|c|c|}
\hline Test & Advantages & Disadvantages \\
\hline \multicolumn{3}{|l|}{ Direct methods } \\
\hline Directly observed therapy & Most accurate & $\begin{array}{l}\text { Patients can hide pills in the mouth } \\
\text { and discard them; impractical for } \\
\text { routine use }\end{array}$ \\
\hline $\begin{array}{l}\text { Measurement of the level of } \\
\text { medicine or metabolite in blood }\end{array}$ & Objective & $\begin{array}{l}\text { Variations in metabolisms and "white } \\
\text { coat" adherence can give a false } \\
\text { impression of adherence; expensive }\end{array}$ \\
\hline $\begin{array}{l}\text { Measurement of the biologic } \\
\text { marker in blood }\end{array}$ & $\begin{array}{l}\text { Objective in clinical trials, can also } \\
\text { be used to measure placebo }\end{array}$ & $\begin{array}{l}\text { Requires expensive quantitative } \\
\text { essays and collection of bodily fluids }\end{array}$ \\
\hline \multicolumn{3}{|l|}{ Indirect methods } \\
\hline $\begin{array}{l}\text { Patient questionnaires, patient } \\
\text { self-reports }\end{array}$ & $\begin{array}{l}\text { Simple; inexpensive; the most useful } \\
\text { methods in the clinical setting }\end{array}$ & $\begin{array}{l}\text { Susceptible to error with increases in } \\
\text { time between visits; results are } \\
\text { easily distorted by the patient }\end{array}$ \\
\hline Pill counts & $\begin{array}{l}\text { Objective; quantifiable; and easy to } \\
\text { perform }\end{array}$ & $\begin{array}{l}\text { Data easily altered by the patient } \\
\text { (e.g. pill dumping) }\end{array}$ \\
\hline Rates of prescription refills & Objective; easy to obtain data & $\begin{array}{l}\text { A prescription refill is not equivalent } \\
\text { to the ingestion of medications; } \\
\text { requires a closed pharmacy system. }\end{array}$ \\
\hline $\begin{array}{l}\text { Assessment of the patient`s } \\
\text { clinical response }\end{array}$ & Simple; generally easy to perform & $\begin{array}{l}\text { Factors other than medication } \\
\text { adherence can affect clinical } \\
\text { response }\end{array}$ \\
\hline Electronic medication monitors & $\begin{array}{l}\text { Precise; results are easily quantified; } \\
\text { tracks patterns of taking medication }\end{array}$ & $\begin{array}{l}\text { Expensive; requires return visits and } \\
\text { downloading data from medication } \\
\text { vials }\end{array}$ \\
\hline $\begin{array}{l}\text { Measurement of physiologic } \\
\text { markers (e.g. heart rate in patients } \\
\text { taking beta-blockers) }\end{array}$ & Often easy to perform & $\begin{array}{l}\text { Marker may be absent for other } \\
\text { reasons (e.g. increased metabolism, } \\
\text { poor absorption, lack of response) }\end{array}$ \\
\hline Patient diaries & Help to correct for poor recall & Easily altered by the patient \\
\hline $\begin{array}{l}\text { When the patient is a child, } \\
\text { questionnaire for caregiver of } \\
\text { teacher }\end{array}$ & Simple; objective & Susceptible to distortion \\
\hline
\end{tabular}

Contrary to prescription/claims studies, studies using self-reported adherence $(n=6)$ showed considerably better adherence rates. Whether this is attributable to actual better adherence, the use of self-reported data, or selection bias could not be disentangled. Although depending on the type of questionnaire and study population, overall selfreported approaches overestimate adherence. ${ }^{47}$ The validity is limited by social desirability bias and by subjective interpretations of responses by the interviewer. ${ }^{48}$ Since only two studies used a validated questionnaire, ${ }^{49,50}$ it is likely that, at least for the other four studies, adherence is somewhat overestimated. In this respect, objective methods would be more valid. Thus far only two small studies used MEMS $(n=17)^{51}$ or pill count $(n=132)^{52}$ data and both showed a high level of medication adherence. 
The discrepancy in medication adherence between population studies (claims/prescriptions) and studies among patients treated in secondary care raises the suggestion that patients under care of a general practitioner behave differently compared to those under care of a rheumatologist. The patients under care by a rheumatologist are likely to have more severe gout, more comorbidities, and might have experienced the negative consequences of not being adherent, all this might lead to better adherence compared to those patients seen by the general practitioner. This idea is supported by two studies who found that patients receiving uric acid lowering therapy from a rheumatologist instead of a non-rheumatologist had better adherence. ${ }^{53,54}$ It should be noted, due to the small sample sizes and difference in method to assess adherence, we cannot formally conclude that patients in secondary care have better adherence. $^{51,52}$

\section{Measuring persistence or adherence}

The overall concept "medication adherence" consists of multiple components, including implementation, non-persistence and overall adherence. In this thesis mainly patient adherence and non-persistence (Chapter 5 and 6 ) and the initiation of uric acid lowering therapy by the general practitioner has been examined (Chapter 7). Which concept of medication adherence is clinically most relevant may depend on the specific disease, the prescribed medication and the clinical outcome of interest. With regard to uric acid lowering therapy in patients with gout, the therapy is usually considered to be lifelong after initiation. When uric acid lowering therapy is initiated because of the frequency of flares, but with absence of subcutaneous or intra-articular tophi, persistence with uric acid lowering therapy might be of primary importance. In patients in which uric acid lowering therapy is started in the presence of tophi, the velocity of tophi reduction is linearly related to serum uric acid concentration. ${ }^{55}$ Thereupon, adherence to uric acid lowering therapy might be equally relevant as persistence, so serum uric acid concentration lower than the saturation point, e.g. less than $4(238 \mu \mathrm{mol} / \mathrm{L})$ or $5 \mathrm{mg} / \mathrm{dL}$ (300 $\mu \mathrm{mol} / \mathrm{L})$ can be achieved.

\section{Factors affecting medication adherence}

Thus far, the results from previous studies and our CPRD study gave a good overview of demographic as well as clinical patient characteristics which were associated with poor medication adherence, although the exact rationale for interrupting or stopping medication usage was seldom studied. Medication adherence is a multidimensional phenomenon determined by the interplay of five sets of factors, all of these "dimensions" play a significant role in patient medication adherence (Figure 1). ${ }^{56}$ 


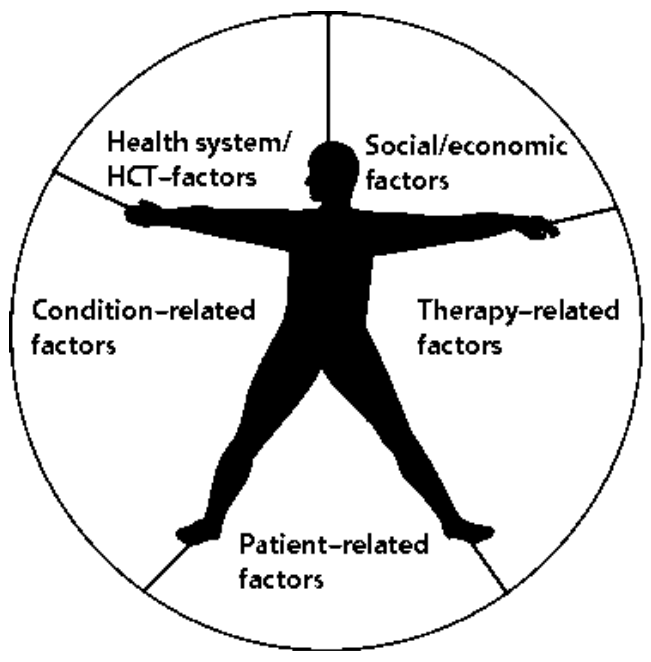

Figure 1. The five dimensions of medication adherence. ${ }^{56}$

One of these dimensions are patient related factors representing the resources, knowledge, attitudes, beliefs, perceptions and expectations of the gout patients and the use of medication. ${ }^{56}$ For example, concerns about using medication might either be of practical nature, such as taking the medicine on a daily basis (forgetfulness), or perceptual nature, fears for actual short and long-term side effects or perceived efficacy of the medication, can influence patients' behaviour. These factors have not been frequently studied ${ }^{50,52,57,58}$ and therefore no conclusion regarding their role in medication adherence can be made.

Other important aspects are related to Health care team and system-related factors. In The Netherlands, as in most other countries, the general practitioner is mainly responsible for the management of gout. The general practitioners in our study indicated that they had a lack of time and had a practical approach concerning adherence to medication and relied on the patient presenting themselves after experiencing a gout flare; the general practitioners themselves showed little concern for monitoring adherence directly. Our CPRD study confirmed that almost $50 \%$ of patients returned to therapy after an initial gap. However, assuming they all had a correct indication to start and to continue longterm uric acid lowering therapy, half of the patients did not return (Chapter 5). Even after restarting therapy, less than 50\% were persistent in the following year. Restarting therapy thus seems not to guarantee better adherence to medication. Other reasons for the limited attention of the general practitioner to adherence could be an overestimation of their own communication skills or the patient's ability to understand the importance of appropriate medication use and lifestyle choices. ${ }^{59}$ Finally, general practitioners may feel that their role in lifestyle and adherence support is of secondary 
concern compared to their role in diagnosis and providing treatment. Thus, the common belief that solely the patient is responsible for taking the medication is misleading and obsolete.

\section{Guidelines for the management of gout}

Our study among general practitioners revealed a large variation in the long-term management of gout, mainly with regard to initiation and duration of uric acid lowering therapy, and initiation of prophylactic treatment. When trying to understand these differences, remarkable contradictions between the recommendations for the long-term management of gout developed by rheumatology societies or the American College of Physicians $(A C P)^{60}$ and Dutch general practitioner ${ }^{61}$ societies were noticed (Table 4).

For example, treatment recommendations for gout developed by rheumatology societies promote a treat to target strategy, which is a serum uric acid concentration below the physiologic threshold of urate crystallization $(<6.8 \mathrm{mg} / \mathrm{dL})$ and below $5 \mathrm{mg} / \mathrm{dL}$ when tophi is present. Whereas, general practitioner and ACP societies promote a treat to symptom (gout flares) strategy. Another discrepancy is present for the initiation of uric acid lowering therapy; rheumatology societies promote the initiation of uric acid lowering therapy, while general practitioner and ACP are more reserved in the initiation of uric acid lowering therapy.

The discrepancy in recommendations has created much debate between both societies. ${ }^{62-64}$ The developers of the $A C P^{60}$ guidelines argue their recommendations are based on verifiable systematic review of published evidence, whereas American Congress of Rheumatology (ACR) ${ }^{65}$ and European League Against Rheumatism (EULAR) ${ }^{66}$ rely, in case of absence of evidence, on expert panel opinions. This latter approach was supported by the argument that published literature may not be adequate in providing sufficient evidence for day-to-day clinical practice.

It is beyond the scope of this thesis to discuss details of the evidence and decision process that resulted in the different recommendations. However, it is of uttermost importance to draft a common agenda with a strategy how to solve the contradicting long-term management advises.

One unresolved issue, not specifically addressed in the current discussion on the different guidelines, is whether gout patients in first and secondary care differ in phenotype and therefore need different care and treatment. Possibly, experiences of general practitioners and other physicians truly differ compared to the experience of the rheumatologist. Moreover, most evidence on management of gout is based on studies conducted in the secondary or even tertiary care. Studies addressing the possible differences in patients that need to be referred to secondary care are needed to resolve this issue. 
$168 \mid$ Chapter 8

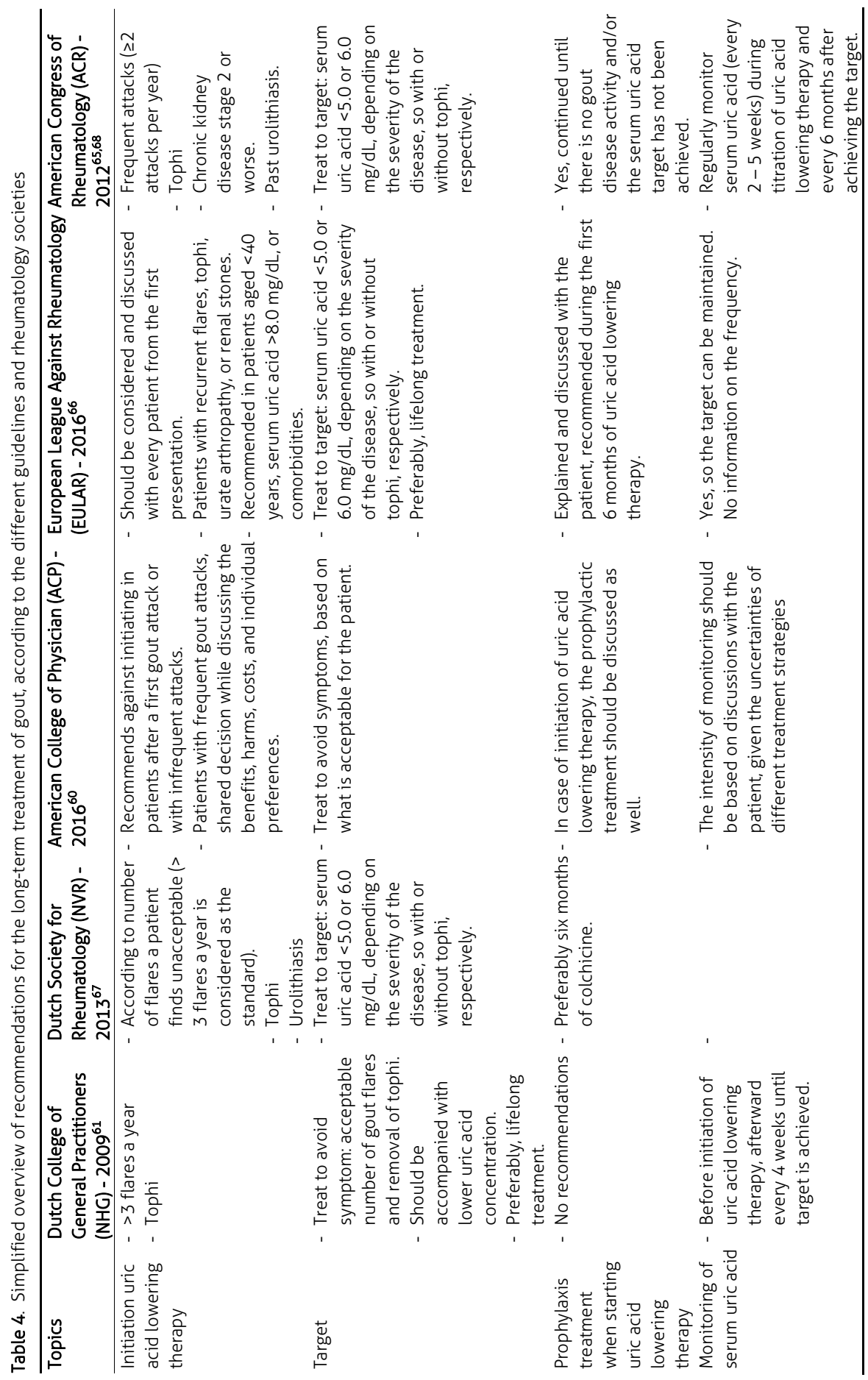




\section{Implications for clinical practice and future directions}

Even in the absence of agreement on when to start uric acid lowering therapy in patients with gout, it cannot be ignored that poor medication adherence is common and seems to have clinical relevance. Therefore, interventions to improve medication adherence would be welcomed. Recently, excellent long-term persistence and adherence on uric acid lowering therapy was achieved when gout patients received individualized education by the nurse. ${ }^{69}$ In parallel to such an intervention, insight into the dynamics and reasons for poor medication adherence are needed to improve adherence and thus the care of gout. Recognizing that there is no consensus yet on the initiation of uric acid lowering therapy, a personalized approach and shared decision-making, might be a path to improve medication adherence. ${ }^{70,71}$

Next, action should be taken to solve the remarkable discrepancies between general practitioners and rheumatologists in the long-term management of gout. Next to tailored education for the provider, evidence based recommendation are required to resolve the ongoing debate on the best strategy in the long-term treatment of gout. The only way to overcome this would be a long-term trial which investigates the outcomes of the two different approaches: treat-to-target and treat-to-avoid symptoms. Such a study should be stratified according to setting, either general practitioner or rheumatologist, to ensure that the outcome is valid for the entire gout population, and not only those seen and treated in secondary care.

\section{OVERALL CONCLUSION}

Our findings suggest that overproduction of uric acid, reflected by studying three different proxies, might be involved in the pathogenesis of hypertension. Further research on the validation of the proxies and confirmation of the hypothesis is required before the clinical relevance can be considered. Furthermore, we showed suboptimal long-term management of gout in population studies. Poor medication adherence in gout patients as well as large variation in general practitioners' long-term gout management contribute to this problem. To ultimately improve the care of gout patients the implementation of adherence interventions and creating clear guidelines are necessary. Important to take into account, patients seen in first or second line might actually need different care; which might result into different recommendation for general practitioners and rheumatologists. 
$170 \mid$ Chapter 8

\section{REFERENCES}

1. Landmesser U, Spiekermann S, Preuss C, et al. Angiotensin II induces endothelial xanthine oxidase activation: role for endothelial dysfunction in patients with coronary disease. Arterioscler Thromb Vasc Biol. 2007; 27(4):943948.

2. Mazzali M, Hughes J, Kim YG, et al. Elevated uric acid increases blood pressure in the rat by a novel crystal-independent mechanism. Hypertension. 2001; 38(5):1101-1106.

3. Richette P, Doherty M, Pascual E, et al. 2016 updated EULAR evidence-based recommendations for the management of gout. Ann Rheum Dis. 2017; 76(1):29-42.

4. Rothenbacher D, Primatesta P, Ferreira A, et al. Frequency and risk factors of gout flares in a large population-based cohort of incident gout. Rheumatology (Oxford). 2011; 50(5):973-981.

5. Doherty $M$, Jansen $T L$, Nuki $G$, et al. Gout: why is this curable disease so seldom cured? Ann Rheum Dis. 2012; 71(11):1765-1770.

6. Sheehan NA, Didelez V, Burton PR, et al. Mendelian randomisation and causal inference in observational epidemiology. Plos Med. 2008; 5(8):e177.

7. Smith GD, Ebrahim S. Mendelian randomization: prospects, potentials, and limitations. Int J Epidemiol. 2004; 33(1):30-42.

8. Freeman B, Powell J, Ball D, et al. DNA by mail: an inexpensive and noninvasive method for collecting DNA samples from widely dispersed populations. Behav Genet. 1997; 27(3): 251-257.

9. Plomin R, Rutter M. Child development, molecular genetics, and what to do with genes once they are found. Child Dev. 1998; 69(4):1223-1242.

10. Gratten J, Visscher PM. Genetic pleiotropy in complex traits and diseases: implications for genomic medicine. Genome Med. 2016;8(1):78.

11. Lim SS, Vos T, Flaxman AD, et al. A comparative risk assessment of burden of disease and injury attributable to 67 risk factors and risk factor clusters in 21 regions, 1990-2010: a systematic analysis for the Global Burden of Disease Study 2010. Lancet. 2012; 380(9859):2224-2260.

12. Tanaka K, Kanazawa I, Yamasaki $\mathrm{H}$, et al. Xanthinuria Type I With a Novel Mutation of Xanthine Dehydrogenase. Am J Med Sci. 2015; 350(2):155-156.

13. Zhou YN, Zhang $X G$, Ding $R$, et al. Using NextGeneration Sequencing to Identify a Mutation in Human MCSU that is Responsible for Type II
Xanthinuria. Cell Physiol Biochem. 2015; 35(6):2412-2421.

14. Curto R, Voit EO, Sorribas A, et al. Mathematical models of purine metabolism in man. Math Biosci. 1998; 151(1):1-49.

15. Lane AN, Fan TW. Regulation of mammalian nucleotide metabolism and biosynthesis. Nucleic Acids Res. 2015;43(4):2466-2485.

16. Puig JG, Mateos FA, Jimenez ML, et al. Renal excretion of hypoxanthine and xanthine in primary gout. Am J Med. 1988; 85(4):533-537.

17. Puig JG, Torres RJ, de Miguel E, et al. Uric acid excretion in healthy subjects: a nomogram to assess the mechanisms underlying purine metabolic disorders. Metabolism. 2012; 61(4):512-518.

18. Loffler W, Grobner W, Medina R, et al. Influence of dietary purines on pool size, turnover, and excretion of uric acid during balance conditions. Isotope studies using 15N-uric acid. Res Exp Med (Berl). 1982; 181(2):113-123.

19. Hosomi A, Nakanishi T, Fujita T, et al. Extra-renal elimination of uric acid via intestinal efflux transporter BCRP/ABCG2. PLoS One. 2012; 7(2):e30456.

20. Matsuo H, Takada T, Nakayama A, et al. ABCG2 dysfunction increases the risk of renal overload hyperuricemia. Nucleosides Nucleotides Nucleic Acids. 2014; 33(4-6):266-274.

21. Uhlen M, Fagerberg L, Hallstrom BM, et al. Proteomics. Tissue-based map of the human proteome. Science. 2015; 347(6220):1260419.

22. Takada $T$, Ichida $K$, Matsuo $H$, et al. ABCG2 dysfunction increases serum uric acid by decreased intestinal urate excretion. Nucleosides Nucleotides Nucleic Acids. 2014; 33(4-6):275281.

23. Yu KH, Luo SF, Tsai WP, et al. Intermittent elevation of serum urate and 24-hour urinary uric acid excretion. Rheumatology (Oxford). 2004; 43(12): 1541-1545.

24. Sennels HP, Jorgensen $H L$, Goetze JP, et al. Rhythmic 24-hour variations of frequently used clinical biochemical parameters in healthy young males--the Bispebjerg study of diurnal variations. Scand J Clin Lab Invest. 2012; 72(4):287-295.

25. Choi JW, Ford ES, Gao X, et al. Sugar-sweetened soft drinks, diet soft drinks, and serum uric acid level: the Third National Health and Nutrition Examination Survey. Arthritis Rheum. 2008; 59(1):109-116. 
26. Choi HK, Liu S, Curhan G. Intake of purine-rich foods, protein, and dairy products and relationship to serum levels of uric acid: the Third National Health and Nutrition Examination Survey. Arthritis Rheum. 2005; 52(1):283-289.

27. Green HJ, Fraser IG. Differential effects of exercise intensity on serum uric acid concentration. Med Sci Sports Exerc. 1988; 20(1):55-59.

28. Houston M, Estevez A, Chumley P, et al. Binding of xanthine oxidase to vascular endothelium. Kinetic characterization and oxidative impairment of nitric oxide-dependent signaling. J Biol Chem. 1999; 274(8):4985-4994.

29. Harrison R. Structure and function of xanthine oxidoreductase: where are we now? Free Radic Biol Med. 2002; 33(6):774-797.

30. Adachi T, Fukushima T, Usami Y, et al. Binding of human xanthine oxidase to sulphated glycosaminoglycans on the endothelial-cell surface. Biochem J. 1993; 289 ( Pt 2):523-527.

31. Radi R, Rubbo H, Bush K, et al. Xanthine oxidase binding to glycosaminoglycans: kinetics and superoxide dismutase interactions of immobilized xanthine oxidase-heparin complexes. Arch Biochem Biophys. 1997; 339(1):125-135.

32. Rouquette $M$, Page $S$, Bryant $R$, et al. Xanthine oxidoreductase is asymmetrically localised on the outer surface of human endothelial and epithelial cells in culture. FEBS Lett. 1998; 426(3):397-401.

33. Vickers $\mathrm{S}$, Schiller $\mathrm{HJ}$, Hildreth $\mathrm{JE}$, et al. Immunoaffinity localization of the enzyme xanthine oxidase on the outside surface of the endothelial cell plasma membrane. Surgery. 1998; 124(3):551-560.

34. Pritsos CA. Cellular distribution, metabolism and regulation of the xanthine oxidoreductase enzyme system. Chem Biol Interact. 2000; 129(12):195-208.

35. Kayyali US, Donaldson C, Huang $\mathrm{H}$, et al. Phosphorylation of xanthine dehydrogenase/ oxidase in hypoxia. J Biol Chem. 2001; 276(17):14359-14365.

36. Caliceti C, Calabria D, Roda A. A new sensitive and quantitative chemiluminescent assay to monitor intracellular xanthine oxidase activity for rapid screening of inhibitors in living endothelial cells. Anal Bioanal Chem. 2016; 408(30):8755-8760.

37. Franklin SS, Thijs L, Li Y, et al. Masked hypertension in diabetes mellitus: treatment implications for clinical practice. Hypertension. 2013; 61(5):964-971.
38. Ohkubo T, Kikuya M, Metoki $\mathrm{H}$, et al. Prognosis of "masked" hypertension and "white-coat" hypertension detected by 24-h ambulatory blood pressure monitoring 10-year follow-up from the Ohasama study. J Am Coll Cardiol. 2005; 46(3):508-515.

39. McCord JM, Fridovich I. The reduction of cytochrome c by milk xanthine oxidase. J Biol Chem. 1968; 243(21):5753-5760.

40. Battelli MG, Bolognesi A, Polito L. Pathophysiology of circulating xanthine oxidoreductase: new emerging roles for a multitasking enzyme. Biochim Biophys Acta. 2014; 1842(9):1502-1517.

41. So A, Thorens B. Uric acid transport and disease. The Journal of clinical investigation. 2010; 120(6):1791-1799.

42. Intengan HD, Schiffrin EL. Vascular remodeling in hypertension: roles of apoptosis, inflammation, and fibrosis. Hypertension. 2001; 38(3 Pt 2):581-587.

43. Mulvany M]. Vascular remodelling of resistance vessels: can we define this? Cardiovasc Res. 1999; 41(1):9-13.

44. Falaschetti E, Mindell J, Knott C, et al. Hypertension management in England: a serial cross-sectional study from 1994 to 2011. Lancet. 2014; 383(9932):1912-1919.

45. Osterberg L, Blaschke T. Adherence to medication. N Engl J Med. 2005; 353(5):487-497.

46. Choo PW, Rand CS, Inui TS, et al. Validation of patient reports, automated pharmacy records, and pill counts with electronic monitoring of adherence to antihypertensive therapy. Med Care. 1999; 37(9):846-857.

47. Shi L, Liu J, Koleva $Y$, et al. Concordance of adherence measurement using self-reported adherence questionnaires and medication monitoring devices. Pharmacoeconomics. 2010; 28(12):1097-1107.

48. Stirratt MJ, Dunbar-Jacob J, Crane HM, et al. Selfreport measures of medication adherence behavior: recommendations on optimal use. Transl Behav Med. 2015; 5(4):470-482.

49. Dalbeth N, House ME, Horne A, et al. Prescription and dosing of urate-lowering therapy, rather than patient behaviours, are the key modifiable factors associated with targeting serum urate in gout. BMC Musculoskelet Disord. 2012; 13:174.

50. Dalbeth $N$, Petrie KJ, House $M$, et al. Illness perceptions in patients with gout and the relationship with progression of musculoskeletal disability. Arthritis Care Res (Hoboken). 2011; 63(11):1605-1612. 
51. de Klerk E, van der Heijde $D$, Landewe $R$, et al. Patient compliance in rheumatoid arthritis, polymyalgia rheumatica, and gout. ] Rheumatol. 2003; 30(1):44-54.

52. Lee S, So MW. Adherence with urate-lowering therapies among male patients with gout in a routine clinical setting. Mod Rheumatol. 2016: 16.

53. Solomon DH, Avorn J, Levin R, et al. Uric acid lowering therapy: prescribing patterns in a large cohort of older adults. Ann Rheum Dis. 2008; 67(5):609-613.

54. Rashid N, Coburn BW, Wu YL, et al. Modifiable factors associated with allopurinol adherence and outcomes among patients with gout in an integrated healthcare system. J Rheumatol. 2015; 42(3):504-512.

55. Perez-Ruiz F, Calabozo M, Pijoan Jl, et al. Effect of urate-lowering therapy on the velocity of size reduction of tophi in chronic gout. Arthritis Rheum. 2002; 47(4):356-360.

56. Sabaté E. Adherence to long-term therapies: evidence for action. World Health Organization; 2003.

57. de Klerk $E$, van der Heijde $D$, Landewe $R$, et al. Patient compliance, in rheumatoid arthritis, polymyalgia rheumatica, and gout. J Rheumatol. 2003; 30(1):44-54.

58. Singh JA, Shah N, Edwards NL. A cross-sectional internet-based patient survey of the management strategies for gout. BMC Complement Altern Med. 2016; 16:90.

59. Ha JF, Longnecker N. Doctor-patient communication: a review. Ochsner J. 2010; 10(1):38-43.

60. Oaseem A, Harris RP, Forciea MA, Clinical Guidelines Committee of the American College of P. Management of Acute and Recurrent Gout: A Clinical Practice Guideline From the American College of Physicians. Ann Intern Med. 2017; 166(1): 58-68.

61. Janssens HJEM, Lagro HAHM, Van Peet PG, et al. NHG-Standaard Artritis. Huisarts en Wetenschap. 2009; 52(9):439-453.
62. FitzGerald JD, Neogi T, Choi HK. Do not let gout apathy lead to gouty arthropathy. Arthritis Rheumatol. 2016.

63. Neogi T, Mikuls TR. To treat or not to treat (to target) in gout. Ann Intern Med. 2017; 166(1):7172.

64. McLean RM. The long and winding road to clinical guidelines on the diagnosis and management of gout. Ann Intern Med. 2017; 166(1):73-74.

65. Khanna D, Fitzgerald JD, Khanna PP, et al. 2012 American College of Rheumatology guidelines for management of gout. Part 1: systematic nonpharmacologic and pharmacologic therapeutic approaches to hyperuricemia. Arthritis Care Res (Hoboken). 2012; 64(10):14311446.

66. Richette P, Doherty M, Pascual E, et al. 2016 updated EULAR evidence-based recommendations for the management of gout. Ann Rheum Dis. 2016.

67. Reumatologie NVR. Richtlijn Jicht. 2013.

68. Khanna D, Khanna PP, Fitzgerald JD, et al. 2012 American College of Rheumatology guidelines for management of gout. Part 2: therapy and antiinflammatory prophylaxis of acute gouty arthritis. Arthritis Care Res (Hoboken). 2012; 64(10): 1447-1461.

69. Abhishek A, Jenkins W, La-Crette J, et al. Longterm persistence and adherence on uratelowering treatment can be maintained in primary care-5-year follow-up of a proof-ofconcept study. Rheumatology (Oxford). 2017.

70. Charles C, Gafni A, Whelan T. Shared decisionmaking in the medical encounter: what does it mean? (or it takes at least two to tango). Soc Sci Med. 1997; 44(5): 681-692.

71. Sandman L, Granger BB, Ekman I, et al. Adherence, shared decision-making and patient autonomy. Med Health Care Philos. 2012; 15(2):115-127. 



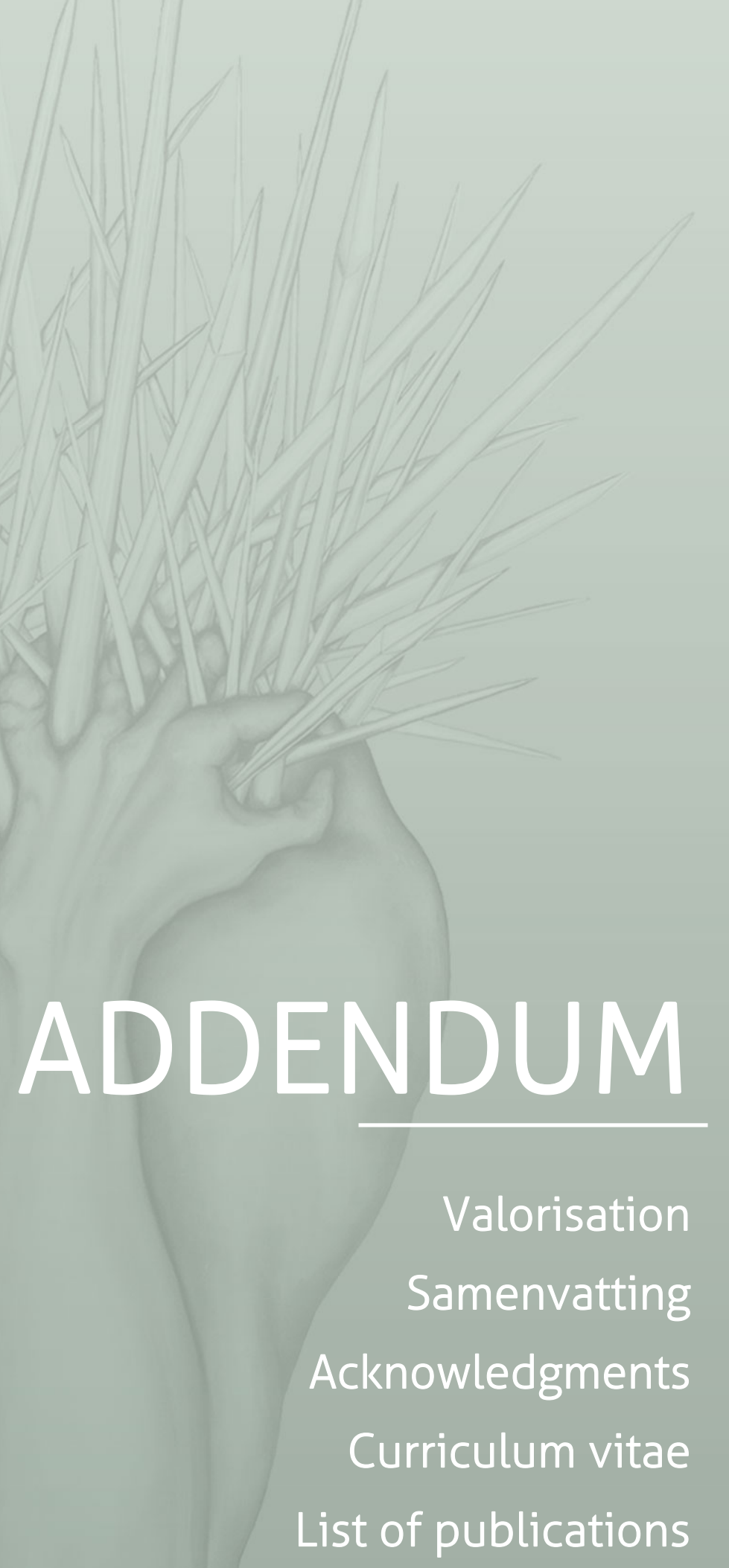





\section{VALORISATION}

An important aspect of research is to ensure its results will have, besides scientific merit, also social and economic value. This addendum describes how society may benefit from the work conducted in this thesis.

Gout is the most prevalent form of inflammatory rheumatic disease and affects $1-2 \%$ of the population worldwide. ${ }^{1}$ The prevalence is particularly high among men, and approximately $7 \%$ of men above 65 are affected. ${ }^{2}$ Hyperuricemia, the most important risk factor for gout, is with a prevalence of $20 \%$ far more prevalent than gout. Recent studies showed that both gout and asymptomatic hyperuricemia have been associated with an increased risk for cardiovascular disease, cardiovascular mortality and all-cause mortality. ${ }^{3-5}$ Moreover, patients with gout or asymptomatic hyperuricemia have higher all-cause health care utilization and cost compared with those without a diagnosis of gout or without hyperuricemia. ${ }^{6-8}$ For example, the estimated all-cause total direct costs was $\$ 16,925$ per elderly gout patient and $\$ 10,590$ for a non-gout patient. ${ }^{9}$ Overall, gout and asymptomatic hyperuricemia impose a significant economic and social burden for society. ${ }^{10}$

While elevated serum uric acid is a clear risk marker for gout, the role of uric acid in the development of high blood pressure (hypertension) and cardiovascular disease remains uncertain. Since there is biological evidence that the production of uric acid might lead to vascular dysfunction and elevated blood pressure it is of interest to investigate this hypothesis. ${ }^{11,12}$ In Part I of this thesis we therefore investigated the role of uric acid production, as an underlying mechanism, in the association between uric acid and blood pressure. Our findings suggest that overproduction of uric acid might be involved in the pathogenesis of hypertension. Although the study outcomes from Part I of this thesis may not directly lead to societal benefit at first, they can increase insight into the association of uric acid with hypertension and cardiovascular disease and may lead to further research initiatives.

Overall the studied proxies need to be validated in biological experiments to establish if they reflect increased uric acid production. Furthermore, the present findings need to be confirmed in other existing datasets. If confirmed, defining the threshold for uric acid overproducers at risk for developing hypertension is required. Finally, randomized controlled trials are needed to examine whether in uric acid overproducers inhibitors of uric acid production reduce (high) blood pressure or prevent the development of hypertension.

If our hypothesis will be confirmed, the identification of uric acid overproducers might be valuable to identify people who are at high risk for developing hypertension and cardiovascular disease. Today only $37 \%$ of the hypertensive patients obtain a well- 
controlled blood pressure. ${ }^{13}$ Even this estimate is conservative; the average expenditure for a hypertensive patient in the United States is about $\$ 1320$ per year. ${ }^{14}$ In other words, uncontrolled blood pressure is a high health burden for the patient and economic burden for society. Therefore, a better understanding of the underlying pathophysiology, the identification of persons at risk and novel pharmacological treatment is of main importance.

In the management of chronic gout, a substantial group of patients need long-term management with uric acid lowering therapy. From the findings of Part $/ /$ of this thesis it appears that poor medication adherence among gout patients is common. We found that less than $40 \%$ of the patients in whom allopurinol treatment is started are adherent to their medication. Moreover, although patients who have stopped therapy are likely to return, the changes of a backlash are as likely.

Assuming that uric acid lowering therapy is initiated for the right reasons, non-adherence may not only lead to a higher health-burden for the patient, but may also lead to an economic burden due to additional health care expenditures in those with more advanced and possibly complicated gout. In order to improve medication adherence insights into factors associated with poor medication adherence should be known. The results of this thesis gave a good overview of demographic as well as clinical patient characteristics which were associated with poor medication adherence. Health care providers should provide extra support to those patients who are at high risk for poor medication adherence. Target groups are younger and 'healthier' patients, including patients with a normal weight, who are not on antihypertensive medication, and do not suffer from comorbidities like diabetes or hypertension. Although we identified patients at high risk, the exact rationale for interrupting or stopping medication usage was seldom studied. More insight into patients' understanding of the disease and medication and involved concerns is needed.

As gout is mainly treated in primary care and as poor medication adherence can be either a patient behaviour or provider directed, we explored the knowledge, illness perceptions and stated clinical practice behaviour of general practitioners when managing gout. The attitude about adherence was surprising since almost all general practitioners prefer to wait for recurrent attacks instead of actively screening for adherence. Moreover, if the problem of non-adherence is not addressed, they might draw the false conclusion that the medication is not effective and change to other, often more expensive, medications. Therefore, general practitioners should be more aware of the problem of non-adherence and take responsibility therein.

Changing clinical practice behaviour is a challenge, next to an educational program for general practitioners on the problem and importance of medication adherence, it is of utmost important to elucidate the general practitioners' opinion on the long-term 
management of gout. Moreover, as discussed in the Discussion section of this thesis, further research should focus more on patients seen in primary care instead of those patients seen in secondary care. Eventually, this teaches us what the best strategy and target is for the long-term management of gout and would provide evidence-based recommendations for the treatment of gout in primary care.

Since non-adherence has consequences for the patient's health, health care provider, and society, medication adherence should be seen as a shared responsibility. It is a reasonable, although not yet proven assumption, that successful long-term management should improve individual's health and reduce the financial burden of gout. Thus far, little is known on the consequences of poor medication adherence, we therefore recommend further studies to investigate the impact of poor medication adherence on the patient's health, quality of life, health care utilization and related costs for society. These data can be the starting point for cost-effectiveness studies into adherence improvement programs.

In the end, the results of this thesis contribute to a better understanding of the role of uric acid in the development of hypertension. However, only after confirmation and when causality is established the societal utilization of the present findings may be feasible. Even if this only accounts for a small proportion of the total risk for hypertension and cardiovascular disease, addressing this may lead to a personalized approach and thereby a better public health. In addition, the results presented in this thesis emphasize the need for more awareness among health care providers for medication adherence. Whereas rheumatologists play an important role in treating the more severe cases of gout, general practitioners are the key player for most gout patients. This should be translated into intervention programs for improving medication adherence in a representative, thus mainly patients treated in primary care, gout population. 


\section{REFERENCES}

1. Wijnands JM, Viechtbauer W, Thevissen K, et al. Determinants of the prevalence of gout in the general population: a systematic review and meta-regression. Eur J Epidemiol. 2015;30:1933.

2. Mikuls TR, Farrar JT, Bilker WB, et al. Gout epidemiology: results from the UK General Practice Research Database, 1990-1999. Ann Rheum Dis. 2005;64:267-72.

3. Feig DI, Kang DH, Johnson RJ. Uric acid and cardiovascular risk. N Engl J Med. 2008;359: 1811-1821.

4. Niskanen LK, Laaksonen DE, Nyyssonen K, et al. Uric acid level as a risk factor for cardiovascular and all-cause mortality in middle-aged men: a prospective cohort study. Arch Intern Med. 2004;164:1546-51.

5. Choi HK, Curhan G. Independent impact of gout on mortality and risk for coronary heart disease. Circulation. 2007;116:894-900.

6. Singh JA, Strand V. Gout is associated with more comorbidities, poorer health-related quality of life and higher healthcare utilisation in US veterans. Ann Rheum Dis. 2008;67:1310-6.

7. Wu EQ, Forsythe A, Guerin A, et al. Comorbidity burden, healthcare resource utilization, and costs in chronic gout patients refractory to conventional urate-lowering therapy. Am J Ther. 2012:19:e157-66.
8. Wu EQ, Patel PA, Mody RR, et al. Frequency, risk, and cost of gout-related episodes among the elderly: does serum uric acid level matter? J Rheumatol. 2009;36:1032-40.

9. Rai SK, Burns LC, De Vera MA, et al. The economic burden of gout: A systematic review. Semin Arthritis Rheum. 2015;45:75-80.

10. Spaetgens B, Wijnands JM, van Durme $C$, et al. Cost of illness and determinants of costs among patients with gout. J Rheumatol. 2015:42:33544.

11. Mazzali M, Hughes J, Kim YG, et al. Elevated uric acid increases blood pressure in the rat by a novel crystal-independent mechanism. Hypertension. 2001;38:1101-6.

12. Mazzali M, Kanellis J, Han L, et al. Hyperuricemia induces a primary renal arteriolopathy in rats by a blood pressure-independent mechanism. Am J Physiol Renal Physiol. 2002;282:F991-7.

13. Falaschetti E, Mindell J, Knott C, et al. Hypertension management in England: a serial cross-sectional study from 1994 to 2011. Lancet. 2014;383:1912-9.

14. Elliott W]. The economic impact of hypertension. J Clin Hypertens (Greenwich). 2003;5(3 Suppl 2):3-13. 


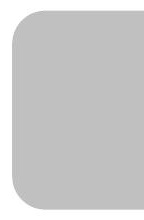




\section{SAMENVATTING}

Jicht is de meest voorkomende reumatologische aandoening en ontstaat door het neerslaan van urinezuur kristallen in en rondom de gewrichten. Dit zorgt voor een pijnlijke ontstekingsreactie in het gewricht of de omringende weefsels. De belangrijkste risicofactor voor jicht is een verhoogde urinezuurconcentratie in het bloed (hyperurikemie), hetgeen wordt veroorzaakt door een verstoring in urinezuurproductie en/of -excretie. Daarnaast gaan jicht en asymptomatische hyperurikemie vaak gepaard met ongunstige cardio-metabole uitkomsten, zoals overgewicht en hoge bloeddruk (hypertensie). Jicht en de bijbehorende nevenaandoeningen vormen een grote last voor zowel de patiënt als voor de gezondheidszorg. Het is daarom van belang meer inzicht te krijgen in de samenhang van jicht en andere aandoeningen en de zorgverlening omtrent jicht.

Het doel van dit proefschrift was tweeledig:

I. Het onderzoeken van de rol van productie van urinezuur in de relatie tussen urinezuur en bloeddruk

II. Het in kaart brengen van therapietrouw aan urinezuur verlagende medicatie bij patiënten met jicht en in de zorg omtrent jicht door huisartsen.

Het onderzoek in dit proefschrift werd uitgevoerd in verschillende observationele studies. Voor het beantwoorden van doelstelling II werd daarnaast de literatuur omtrent medicatie inname systematisch bestudeerd en werden huisartsen ondervraagd.

\section{DEEL I: URINEZUUR EN BLOEDDRUK: DE ROL VAN URINEZUURPRODUCTIE}

De laatste decennia is er veel belangstelling voor de rol van urinezuur bij de ontwikkeling van hoge bloedruk. De oorzakelijkheid van het verband tussen urinezuur en bloeddruk is omstreden en de mogelijke onderliggende mechanismen zijn onduidelijk. Tot dusver is alleen het verband tussen urinezuurconcentraties en bloeddruk bestudeerd, en ontbrak onderzoek dat onderscheid maakte tussen de oorzaak van een verhoogd urinezuur, namelijk verhoogde productie of verminderde excretie. Er zijn aanwijzingen dat niet alleen het urinezuur zelf, maar ook de oxidatieve stress die ontstaat in de laatste fase van de productie van urinezuur verantwoordelijk is voor de nadelige invloed op bloeddruk. De oxidatieve stress die vrijkomt, kan mogelijk de perifere weerstand in de bloedvaten vergoten wat kan leiden tot een verhoogde bloeddruk.

De hypothese die getoetst wordt in deel I van dit proefschrift is dan ook dat een verhoogd productie van urinezuur, en niet alleen het urinezuur zelf kan leiden tot een hogere bloeddruk. Aangezien het vaststellen van urinezuurproductie niet uitvoerbaar is in grotere studies, hebben we drie verschillende maten als proxy voor urinezuurproductie gebruikt. Hieronder wordt een overzicht gegeven van de belangrijkste bevindingen en conclusies. 
$184 \mid$ Chapter 9

\section{Hoofdstuk 2: variatie in het xanthine oxidoreductase gen}

Binnen de Vlaamse FLEMENGHO en de Europese EPOGH studie onderzochten wij de associatie tussen varianten van het xanthine oxidoreductase (XOR) gen en bloeddruk. Het $X O R$ gen codeert voor het enzym xanthine oxidoreductase (XOR) dat verantwoordelijk is voor het aanmaken van urinezuur. Daarbij keken wij of variaties van het XOR gen, als proxy voor 'urinezuur productie', geassocieerd waren met het risico op hypertensie en bloeddrukverandering over de tijd. De resultaten laten zien dat drie van de 25 onderzochte varianten in het gen samenhingen met een grotere toename in bloeddruk en een groter risico op hypertensie. Urinezuurconcentraties in het bloed hingen niet samen met variaties van het XOR gen.

\section{Hoofdstuk 3: ratio's van verschillende purine metabolieten}

Vervolgens onderzochten we de associatie tussen urinezuurconcentratie, urinezuurproductie en bloeddruk bij kinderen van de KOALA studie. In het bloed van deze 7-jarige kinderen hebben we de concentratie van urinezuur en de purines xanthine en hypoxanthine gemeten, en gekeken of deze samenhingen met een hogere systolische en diastolische bloeddruk. Ditmaal dienden ratio's van de verschillende metabolieten als proxy voor activiteit van het XOR enzym. Het hebben van een hogere urinezuur/xanthine en xanthine/hypoxanthine ratio, wijzend op een hogere XOR enzym activiteit, hing samen met een hogere diastolische bloeddruk. Daarnaast hing een hogere concentratie van urinezuur samen met een hogere diastolische bloeddruk. Dit suggereert dat al op jonge leeftijd zowel een verhoogde XOR activiteit als een hogere urinezuurconcentratie samenhangt met een hogere diastolische bloeddruk.

\section{Hoofdstuk 4: urinezuur excretie in urine}

Binnen De Maastricht Studie keken wij wederom naar de relatie tussen urinezuurproductie en bloeddruk. Ditmaal werd "overproductie" van urinezuur gedefinieerd op basis van de mate van urinezuurexcretie in urine. De aanwezigheid van een hogere concentratie urinezuur, bleek inderdaad samen te hangen met een hoger gemiddelde arteriële bloeddruk en de aanwezigheid van hypertensie.

Concluderend, de bevindingen van hoofdstuk 2 - 4 suggereren dat een hoge urinezuurproductie samenhangt met een hogere bloeddruk. Het effect was vooral zichtbaar in de bloeddrukcomponenten die de perifere weerstand reflecteren, oftewel diastolische bloeddruk en de gemiddelde arteriële bloeddruk. Of een langdurig verhoogde urinezuurproductie uiteindelijk zal lijden tot vaatstijfheid dient verder te worden onderzocht. De resultaten van deze studies geven aanleiding tot vervolgonderzoek dat zich richt op de validatie van de bestudeerde proxies en het verder bevestigen van de hypothese, gevolgd door het bepalen van de klinische relevantie. Bijvoorbeeld welke afkapwaardes voor productie dienen te worden 
gehanteerd om te bepalen of iemand een verhoogd risico heeft op een hypertensie en cardiovasculaire ziektes en of het zinvol is XOR remmers voor te schrijven om urinezuurproductie te verminderen bij patiënten die geen jicht hebben.

\section{DEEL II: THERAPIETROUW BIJ PATIËNTEN MET JICHT EN JICHTMANAGEMENT DOOR DE HUISARTS}

Jicht wordt beschouwd als een goed behandelbare ziekte. Een acute jichtaanval wordt behandeld met onstekingsremmers en in geval van chronische jicht is het verlagen van urinezuurconcentraties aanbevolen. Jicht wordt als chronisch beschouwd indien het recidiverend is of bij aanwezigheid van jichtknobbels, ook wel tofi genoemd. Ondanks de goede behandelmogelijkheden lijden veel patiënten aan recidiverende jicht, waaruit blijkt dat de behandeling niet optimaal is. Om de zorg omtrent jicht te verbeteren is het relevant de barrières van zowel de patiënt als behandelaar in kaart te brengen.

$\mathrm{Bij}$ veel chronische aandoeningen is het niet trouw gebruiken van medicatie een veelvoorkomend probleem. Therapie-ontrouw kan zorgen voor suboptimale controle van de ziekte, met alle nadelige gevolgen van dien. Eerder onderzoek wees uit dat therapieontrouw vaak voorkomt bij patiënten met jicht. Jongere patiënten en patiënten die niet ook bekend zijn met andere chronische aandoening zoals hypertensie of diabetes hebben een verhoogd risico om therapie-ontrouw te worden. Echter, het merendeel van deze studies is uitgevoerd in de Verenigende Staten en maak gebruik van verzekeringsdata. Of deze gegevens representatief zijn voor de jichtpatiënt in andere wereldregio's dient nader te worden onderzocht. Tevens ontbreekt er een overzicht van factoren die van invloed zijn op therapie-ontrouw.

Naast de patiënt speelt ook de huisarts een belangrijke rol bij de behandeling van jicht. Het handelen van de huisarts is afhankelijk van (i) de kennis over de pathofysiologie, oorzaken, en symptomen van jicht en (ii) de persoonlijk ideeën die de huisarts heeft over jicht (ziekteperceptie). De complexe interactie tussen kennis, ziekteperceptie en het daadwerkelijk handelen van de huisarts vraagt erom om gelijktijdig onderzocht te worden. Echter, dit ontbreekt nog.

Deel I/ van dit proefschrift richt zich daarom op het onderzoeken van therapietrouw bij jichtpatiënten die urinezuurverlagende medicatie voorgeschreven kregen. We keken naar de verschillende fases van therapietrouw: het starten, de uitvoering, en het stoppen of onderbreken van medicatie-inname. Daarnaast onderzochten we of de bevindingen van therapietrouw verschillend waren bij het gebruik van verschillende meetmethoden, bijvoorbeeld wanneer therapietrouw bepaald was aan de hand van het afhaalbewijs van de medicatie bij de apotheek, zelf-rapportage, of het elektronisch monitoren van het openen en sluiten van medicatiepotjes etc. Tot slot onderzochten we de kennis, ziekteperceptie en het daadwerkelijk handelen van de huisarts omtrent de zorg van jicht. Hieronder wordt een overzicht gegeven van de belangrijkste bevindingen en conclusies. 


\section{Hoofdstuk 5: therapietrouw bij jichtpatiënten}

Binnen de huisartsendatabase 'Clinical Practice Research Datalink' (CPRD) uit het Verenigd Koninkrijk bestudeerden we therapie-ontrouw bij jichtpatiënten die allopurinol voorgeschreven kregen, de meest voorgeschreven urinezuurverlagende medicatie. Meer dan $50 \%$ van de patiënten was therapie-ontrouw. Slechts één-derde van de patiënten gebruikte meer dan $80 \%$ van de voorgeschreven medicatie gedurende de tijd dat patiëntgegevens aanwezig waren in het databestand (gemiddeld 5,5 jaar). Daarnaast onderbrak $40 \%$ van de patiënten hun medicatiegebruik met minimaal 90 dagen in het eerste jaar, oplopend tot bijna $60 \%$ in de eerste 5 jaar. Van deze patiënten herstartte bijna de helft, maar ondanks dit stoptte meer dan de helft wederom in het eerste jaar na herstart. Vrouwen en rokers hadden een verhoogd risico om therapie-ontrouw te worden, terwijl patiënten die antihypertensiva voorgeschreven kregen of leden aan overgewicht, dementie, diabetes, hoog cholesterol, en/of depressie vaker therapietrouw waren.

\section{Hoofdstuk 6: systematische literatuur weergave van therapietrouw bij jichtpatiënten}

In dit hoofdstuk wordt een systematisch literatuuronderzoek en meta-analyse naar therapietrouw bij jichtpatiënten gepresenteerd. In totaal werden 23 observationele studies geïdentificeerd. Verschillende meetmethodes van therapietrouw en kenmerken van studiepopulaties werden bestudeerd om te achterhalen of zij een deel van de therapietrouw konden verklaren. Wij zagen dat de mate van therapietrouw sterk samenhing met de gebruikte meetmethode. De waargenomen therapietrouw was velen malen hoger bij het gebruik van elektronisch monitoren van medicatiepotjes, het tellen van pillen door de behandelaar of zelf-rapportage, dan wanneer voorschrijf gegevens van de apotheek / huisarts werd gebruikt. Of dit enkel komt door de andere meetmethode of doordat andere meetmethodes ook vaak gepaard gingen met andere patiëntenpopulaties, zoals jichtpatiënten onder behandeling bij de reumatoloog in plaats van bij een andere behandelaar, kon niet worden afgeleid. De 13 studies die therapietrouw definieerden als "ten minste $80 \%$ van de observatietijd was gedekt met medicatie" werden samengenomen en lieten zien dat gemiddeld genomen nog geen $50 \%$ van de patiënten therapietrouw was. De mate van therapie-ontrouw in studies uitgevoerd in de Verenigde Staten waren vergelijkbaar met de resultaten van studies uit andere wereldregio's. Vijf studies onderzochten of patiënten hun medicatiegebruik onderbraken, hieruit bleek dat meer dan 50\% van de patiënten hun therapie onderbraken met ten minste 30 dagen.

Therapietrouw hing samen met lagere urinezuurconcentraties. Daarnaast was er overtuigend bewijs dat patiënten die ouder zijn, lijden aan meerdere chronische aandoeningen, of lijden aan diabetes en/of hypertensie beter therapietrouw zijn. De reden voor therapie-ontrouw was slechts in enkele studies bestudeerd en hier konden geen conclusies aan worden verbonden. 


\section{Hoofdstuk 7: management van jicht door de huisarts}

Tot slot werden zowel het kennisniveau als de percepties van huisartsen over de ziekte jicht bestudeerd. Hierbij maakten wij gebruik van bestaande vragenlijsten en aanvullende verdiepende vragen. De kennis over de pathofysiologie, symptomen en de behandeling van acute jicht was nagenoeg uitstekend onder huisartsen. Echter, wij vonden een grote variatie betreft de zorg van chronische jicht. Met name op het gebied van leefstijladvies, het voorschrijven van urinezuurverlagende medicatie en de bijkomende controle van therapietrouw en urinezuurbepaling in het bloed. Een gebrek aan tijd en het neerleggen van de verantwoordelijkheid bij de patiënt waren hiervoor voornaamste reden.

Concluderend, de resultaten van hoofdstuk 5 - 7 laten zien dat management van jicht nog veel te wensen over laat. Een meerderheid van de patiënten was therapie-ontrouw in het nemen van urinezuurverlagende medicatie. Echter, we vonden grote verschillen in de mate van therapietrouw bij de verschillende meetmethodes. Of dit kwam door de gebruikte methode, studieduur of -populatie konden we niet achterhalen. Op basis van onze bevindingen adviseren wij dat toekomstige studies kwantitatief en kwalitatief onderzoek combineren. Zo kan meer inzicht in de dynamiek van medicatiegebruik worden verkregen en kan de reden achter stoppen en herstarten worden achterhaald.

Ten slotte zagen we dat ondanks de goede kennis van de huisarts over de pathofysiologie, oorzaken en symptomen van jicht de lange termijn zorg vaak onvoldoende. Duidelijke richtlijnen voor de lange-termijn zorg van jicht zijn hierbij belangrijk. Bij het bewerkstelligen van dit doel is het belangrijk te realiseren dat de gegeven zorg van de huisarts en reumatoloog van elkaar kunnen verschillen. De patiënt onder behandeling bij de huisarts heeft mogelijk een ander ziekteverloop en daarom andere zorg nodig dan de patiënt onder behandeling bij een reumatoloog. De richtlijnen voor de huisarts zouden dan ook gebaseerd moeten worden op wetenschappelijk onderzoek bij eerstelijns jichtpatiënten en niet, wat nu meestal het geval is, op patiënten uit de tweedelijns zorg. 



\section{ACKNOWLEDGMENTS}

Aangekomen bij het laatste en waarschijnlijk meest gelezen hoofdstuk van mijn proefschrift; het dankwoord. Het spreekt voor zich dat ik dit proefschrift nooit alleen had kunnen schrijven. Sterker nog, niets van dit alles was mogelijk geweest zonder de hulp van velen. Ik wil dan ook iedereen bedanken voor hun bijdragen.

Allereerst gaat mijn dank uit naar mijn promotieteam, prof. A. Boonen, prof. I. Arts en prof. C. Stehouwer. Ik kan jullie niet genoeg bedanken voor de vrijheid en het vertrouwen die jullie mij hebben gegeven om het laatste 1,5 jaar op afstand te werken.

Lieve Annelies en Ilja, wat ben ik blij dat ik onder jullie begeleiding heb mogen promoveren. De twee verschillende leerstoelen maakten dat ik me tijdens mijn promotie zowel op klinisch als epidemiologisch vlak kon ontwikkelen. Jullie passie voor onderzoek, arbeidsethos en persoonlijke interesse zorgden voor een fijne samenwerking. Jullie zijn twee power-vrouwen en onze samenwerking ga ik zeker missen.

Annelies, ik heb bewondering voor je manier van leidinggeven. Met je enthousiasme voor onderzoek en de kliniek, je integriteit en persoonlijke aandacht wist je mij altijd te motiveren. Je nieuwsgierigheid zorgde ervoor dat we zelden via een rechte lijn op ons doel afstoomde. Ja, dit kostte veel tijd, maar wat heb ik er veel van geleerd en plezier aan beleefd.

Ilja, het doen van de eerste analyses, het schrijven van rebuttals, het presenteren van resultaten op een congres, dit alles en nog veel meer heb ik van jou geleerd! Jouw stijl door in de beginfase alles goed te willen uitzoeken en naarmate het einde nadert steeds pragmatischer te worden, is voor mij goud waard. Naast hard werken was er meer. Je kwam mij in Zweden opzoeken, dit ging gepaard met glaasjes wijn, lekker eten en mooie wandelingen, wat een feest!

Coen, dat je allereerste zin tijdens onze meetings standaard "Wat kan ik voor je betekenen?" was, zegt veel over jou als persoon en je stijl van begeleiden. Je begeleiding was vanaf de zijlijn, maar ik kon met vragen altijd bij je terecht waarna ik binnen no time antwoord kreeg zodat ik weer verder kon. Eerst waren onze gesprekken vooral werk gerelateerd, maar naarmate de tijd vorderde bediscussieerden we het nut van een Zweedse fika. Bedankt voor de fijne samenwerking en het vertrouwen.

Leden van de beoordelingscommissie, prof. J. Metsemakers, prof. L. Jacobsson, prof. P. de Leeuw, dr. A. Dehghan en dr. S. Meex, graag wil ik jullie bedanken voor de genomen tijd en moeite om mijn proefschrift te beoordelen op wetenschappelijke kwaliteit. 
Deze thesis was niet tot stand gekomen zonder de inzet van vele coauteurs. Een aantal van hen wil ik graag in het bijzonder bedanken.

Prof. Jan Staessen, dr. Lutgarde Thijs, and dr. Erika Salvi thank you for your cooperation in the FLEMENGHO project. You all taught me a lot about conducting genetic research, programming in SAS and scientific writing. These experiences formed a solid foundation for the rest of my research work. Also thanks to the other colleagues who contributed to the data collection and feedback on the manuscript.

Carel en Wieke, bedankt voor al het werk wat jullie mij uit handen hebben genomen bij het analyseren van de bloeddrukdata van de KOALA Studie. Frank, Andrea en Patrick, bedankt voor het advies bij het uitvoeren van de CPRD-analyses en het efficiënt schrijven van een artikel, hier heb ik veel van geleerd. En tot slot Marloes, bedankt voor het scannen van de bijna 2000 abstracts. Dit was een hoop werk wat zelfs bijna voor niks was geweest. Hopelijk wordt onze "geüpdatete" versie met enthousiasme ontvangen. Bedankt voor de fijne samenwerking.

Gedurende mijn promotie had ik twee werkplekken, De Maastricht Studie en de afdeling Reumatologie. Dat betekent twee keer zoveel vergaderingen en PhD-meetings, maar ook twee keer zoveel vlaai, teamuitjes en gezelligheid. Collega's van De Maastricht Studie, de groep is inmiddels dusdanig groot dat dit te veel mensen zijn om persoonlijk te bedanken. Maar allen bedankt voor de gezelligheid die er naast het werk altijd was. Ik heb het als bijzonder ervaren om deel uit te mogen maken van zo'n grootschalig onderzoeksproject. Collega's van de Reumatologie, bedankt dat ik bij jullie op de afdeling heb mogen promoveren en voor de gezelligheid op het werk en tijdens congressen.

De secretaresses Yvonne, Marian en Claudia, bedankt dat jullie in de overvolle agenda's van Annelies en Ilja altijd een plaatsje voor mij vrij konden maken. In de loop der jaren ben ik erachter gekomen dat dit allesbehalve een gemakkelijk klus is geweest. Bedankt!

De meeste tijd heb ik doorgebracht op kantoor met mijn mede-promovendi van de Reuma-afdeling.

Mij werd vaak gevraagd: "Is zo'n kantoorbaan en vier jaar met hetzelfde onderwerp bezig zijn nou niet saai?". Je zou zeggen van wel maar het tegendeel is waar. Op de Reumaafdeling zou een scriptschrijver van GTST inspiratie op kunnen doen. Het was vaak lachen geblazen, oftewel een feestje om met jullie op te mogen trekken. We hebben ons goed geamuseerd tijdens congressen, stapavonden, carnaval, skivakantie, en natuurlijk midzomer nacht in Tjärnö (het is een wonder dat de Homo sapiens op het eiland deze avond hebben overleefd). Andrea, Antje, Bart, Casper, Carmen, Dirk, Ellis, Frans, Ivette, Joost, José, Lisanne, Mayke, Michiel en Simon bedankt voor de leuke tijd. 
Mijn kamergenoten Ellis en Bart, jullie hebben de afgelopen jaren voor de meeste afleiding gezorgd. Fijn dat een promotietraject meer inhoudt dan je eigen onderwerp en ik nu ook deskundig ben op het gebied van honden, cavia's en kattennamen (om maar een voorbeeld van de opgedane kennis te noemen).

Ellis, ik ken weinig mensen die zo positief en ongecompliceerd zijn als jij. Je gastvrijheid en gezelligheid zijn ongekend, onwijs bedankt dat ik altijd bij jou en Elton heb mogen crashen tijdens mijn logeerweekjes in Maastricht. Jullie zijn schatten, bedankt!

New colleagues at the Rheumatology department in Gothenburg, it is great to work in such a nice team. Mats and Lennart, thank you for giving me the opportunity to work at your research group. The nice atmosphere and the possibility to participate in different project makes that I really enjoy my work. Thank you!

Of familie en vrienden hebben bijgedragen aan mijn promotie is voor mij geen vraag maar een gegeven. Ook al woonde ik de eerste jaren van mijn promotie in het "verre" zuiden, dankzij jullie gastvrijheid (en de NS) kon ik vele weekenden nog met jullie doorbrengen. Gelukkig waren jullie gezelligheid en betrokkenheid ook "grensoverschrijdend". Jullie bezoekjes aan Tjärnö waren dan ook fantastisch!

Kristy, al 28 jaar ga jij door het leven als mijn buurmeisje. Ook al is de afstand in de loop der jaren groter geworden, toch lukt het ons om elkaar jaarlijks te treffen. In hoog tempo kletsen we bij en worden de fijne jeugdherinneringen opgehaald. Renske, mijn allerbeste schoolvriendin waarmee ik 10 jaar in de schoolbanken heb gezeten, samen knikkeren tot aan de tentamenfeestjes. Ook al zien we elkaar al jaren niet meer dagelijks, als we elkaar zien is het binnen no time weer als vanouds. Ik hoop dat we tot aan het bejaardentehuis nog samen mogen scrabbelen (dan zal ik voor een speciale editie van het groene boekje zorgen). Anoek, we waren nog meubels aan het shoppen bij de Ikea toen jullie al langs kwamen in Zweden. Lekker spontaan, wat tekenend is voor al onze bij-klets-dates. Succes met jullie bouwproject, respect!

De mannen van Gelselaar, jullie zijn altijd in voor een mooi (tent)feestje. Een bezoek aan de Achterhoek gaat je dan ook niet in de koude kleren zitten. Ook al zijn Wouter en ik de aparte vogels (uuhm Vischen) van het stel, gelukkig kunnen we altijd zo weer aansluiten en er een groene unit op drinken.

Vrienden van BT, samen studeren in die mooie stad achter de duinen, feestjes op het strand en in de stad, wintersport, de Batavierenrace, Amstel Gold fietsen, pasta avonden aan de Boomsluiterskade, terrasjes pakken, theeleuten, het was genieten! Van de lesstof kan ik me (helaas) niet veel herinneren, maar van de leuke tijd des te meer. Marijke en Sjoerd, het wonen in het Bezuidenhuis was de fundering van mijn studententijd! Werkelijk wat was het soms een gekkenhuis, met plezier en zelfs de nodige heimwee kijk ik terug op onze avondjes in de woonkamer en middagen in de tuin. Zonde op 
zondagavond, woensdag naar Noodlanding en donderdag vertrouwd stappen in Den Haag. Wat hadden we een goede conditie! Ik had hier niets van willen missen.

Urosianen, mijn studententijd werd in Maastricht stijlvol afgesloten. Ondanks de weinige college-uren stond mijn agenda alsnog bomvol met alle Uros-activiteiten. Trainingen, ledenweekenden, hardlopen in Parijs, eten op de baan, borrels in de stad, we waren er maar druk mee!

Elian, zet ons bij elkaar en het aantal decibels vliegt omhoog. We hebben vaak te horen gekregen:

"Are you sisters?"

"Uuuh.... Nee"

"But you look like sisters"

Zussen van elkaar zijn is leuk, maar een goede vriendin als jij zou ik niet willen missen. Net zoals onze vakanties naar Kenya, Marokko en Algonquin (in the best time of the year, lucky us), het is een wonder dat we de bus weten te halen en het iedere keer weer overleven!

Celeste en Stefan, 2017 zijn we spetterend gestart met een goed feestje in een tankstation van Düsseldorf. Een mooie avond die ik niet snel zal vergeten. Samen hardlopen, etentjes, wintersportvakanties in Oostenrijk en Noorwegen, van mij mogen er nog velen activiteiten volgen.

Goudsnikkels, wat bof ik toch om zulke leuke jonkies als vriendinnen te hebben. De vakanties in Schotland, m'n benen doen er nog zeer van maar gelukkig heb ik er geen blaren aan over gehouden (rara hoe kan dat....) en Portugal zijn memorabele hoogtepunten. Ondertussen is ieder van ons een eigen weg in geslagen en meer in het buitenland dan in Nederland, maar gelukkig weten wij elkaar altijd weer te vinden! Shotjes, de beertjes en de geitjes van Annie, Sinterkerst, ouwehoeren totdat we erbij neervallen, wat zijn we toch een Goudsnikkels!

From Maastricht to Tjärnö was a nice change of scenery. I could not have wished for a better place to write and finish my $\mathrm{PhD}$.

Tjärnö people, thank you all for the nice fika's, you made me feel very welcome. Your enthusiasm for your work is inspiring. Next to working on my "own" project I learned the importance of the small snail Littorina littorea, cultivation of seaweeds, and the defense mechanism of Zostra. Overall, in marine sience everything depends and interacts with the salinity, temperature and $\mathrm{pH}$ of the ocean.

But luckily there was more than work, Allice and Joel, Friederike and Oisín, Ella and Kristy, Stina and David, Ylva and Evan, thank you all for the great time. Cross-country skiing while it is $-20^{\circ}$ (oh dear did we really did that, the happiness must have saved us), barbeques at the beach, Aquavit during Midsommerafton, game nights, food parties, life is beautiful at Tjärnö. Tack så mycket. 
In het bijzonder natuurlijk dank aan mijn paranimfen Joost en Famke.

Joost, naast een behulpzame collega was je vooral iemand om mee te kletsen, te lachen en te stappen. Boodschappen doen tijdens de wintersport is nooit meer zo avontuurlijk geweest, kun je mij vertellen wat we gekocht hebben... ik kan me nog wel een "kijkje" in het vriesvak herinneren. Ps, vergeet je je luchtgitaar en zakdoek niet! Deze attributen mogen op mijn promotiefeest natuurlijk niet ontbreken.

Famke, vind je het goed als ik de statistiek- en epidemiologievragen aan jou doorspeel? Ik hoor je al zeggen, "Meneer de opponent wist u dat..." met je leuke feitjes en weetjes weet je zo de tijd te vullen. Superhandig toch... Samen hebben we veel $n=2$ studies uitgevoerd, appelflappen eten voor de wedstrijd werkt niet prestatie bevorderend (ik vind het gek!), maar of sprintend vertrekken nou ook nadelig is... Further research needed. Fijn dat jullie naast mij willen staan deze dag, we gaan er een mooi feest van maken!

Lieve Hank, Ad en Joost, vanaf dag één voel ik me bij jullie thuis. De bezoekjes aan de Achterhoek zijn voor mij net minivakanties; rondhuppelen in m'n pyjama, in de tuin onder het genot van een home-made appeltaart bij kletsen en tot slot 's avonds een potje ballenjagen, wat wil een mens nog meer. Jullie staan altijd voor ons klaar en het is fijn om te weten dat jullie achter ons staan. Ik had me geen fijnere familie kunnen wensen.

Willem, Gijs en Jaap, wat een geluk dat ik drie grote broers heb. Aan tafel ging het dan ook over voetbal in plaats van over vrouwenzaken, die er volgens jullie toch niet toe deden. Janneke, Floor en Andrea, jullie zorgen voor veel extra gezelligheid binnen Huize Scheepers. Houd die mannen onder de duim en zorg goed voor elkaar! De grote afstand heeft er gelukkig niet toe geleid dat we minder betrokken zijn bij elkaars leven. De komst van mijn stoere neefjes Julian, Jacob en Sietse maken ons contact nog intensiever. Bedankt lieve snotaappies! Gijs en Floor, nog een speciaal woord voor jullie, super dat ik altijd bij jullie terecht kan. Lief!

Papa en mama, ook al wonen we niet bij elkaar om de hoek, jullie zijn nog steeds nauw betrokken bij mijn leven. Het is fijn om te zien dat jullie op een afstandje altijd met Wouter en mij mee feesten, maar wat kijk ik ernaar uit om deze dag samen met jullie te vieren. Alles wat ik van jullie heb geleerd en meegekregen is de afgelopen jaren goed van pas gekomen. Tot slot lieve papa, onze fietsvakanties waarin we discussieerden over politiek en 's avonds een bord pasta aten in een Belgisch eetcafé zijn herinneringen die ik koester.

Lieve Visch, min skat, ik ben zo blij met jou dat ik er een heel boek aan kan wijden. Maar ik zal het ditmaal kort houden, je bent de liefste, leukste, en meest aantrekkelijke man die ik ken. Je kijk op de wereld, zorgzaamheid en nuchterheid is iets wat mij iedere dag weer gelukkig maakt. Of het nou fietsend door Kyrgyzstan, langlaufend door Noorwegen, of "gewoon" thuis in Zweden is, ik hoop dat het fijne leven met jou oneindig mag zijn. Jag älskar dig. 



\section{CURRICULUM VITAE}

Lieke Scheepers was born on June $20^{\text {th }} 1988$ in Roosendaal, The Netherlands. In 2005 , she graduated from secondary school at the Norbertuscollege in Roosendaal, which was followed by a one-year exchange program to Australia. Afterwards, she studied Human Kinetic Technology at The Hague University of Applied Sciences and obtained her Bachelor of Engineering degree in 2010. During her Bachelor Program, she performed an internship at the orthopedic workshop in Ghana (2008).

After travelling and cycling across Asia and Eastern-Europe for a year, Lieke started her Master in Public Health - Epidemiology at Maastricht University in 2011. During the Master Program, she did an internship at the department of Epidemiology to investigate the association between the intestinal microbiota composition and weight development in children under supervision of prof. Ilja Arts and dr. John Penders.

In January 2013, Lieke started her PhD project at the department of Rheumatology at Maastricht University Medical Center and at the Care and Public Health Research Institute (CAPHRI) at Maastricht University. The research described in this thesis was conducted under supervision of prof. Annelies Boonen, prof. Ilja Arts and prof. Coen Stehouwer. During her PhD project, she also worked at the research center of The Maastricht Study where she participated in data collection.

Currently, she is appointed as a postdoctoral research fellow at the department of Rheumatology at Gothenburg University in Sweden. Under supervision of prof. Lennart Jacobsson and dr. Mats Dehlin she will continue her research on gout. 



\section{LIST OF PUBLICATIONS}

L.E.J.M. Scheepers, A. Boonen, P.C. Dagnelie, M.T. Schram, C.J.H. van der Kallen, R.M.A. Henry, A.A. Kroon, C. D.A. Stehouwer, I.C.W. Arts. Uric acid production and blood pressure: exploring the role of uric acid production in The Maastricht Study. (accepted for publication in Journal of Hypertension)

L.E.J.M. Scheepers, A. Boonen, W.D. Pijnenburg, J. Bierau, C.D.A. Stehouwer, J.A. Staessen, C. Thijs, I.C.W. Arts. Associations of plasma uric acid and purine metabolites with blood pressure in children: the KOALA Birth Cohort Study. Journal of Hypertension, 2017 May;35(5):982-993.

L.E.J.M. Scheepers, F. Wei, K. Stolarz-Skrzypek, S. Malyutina, V. Tikhonoff, L. Thijs, E. Salvi, C. Barlassina, J. Filipovský, E. Casiglia, Y. Nikitin, K. Kawecka-Jaszcz, P. Manunta, D. Cusi, A. Boonen, J.A. Staessen, I.C.W. Arts. Xanthine oxidase gene variants and their association with blood pressure and incident hypertension: a population study. Journal of Hypertension, Nov 2016; 34:2147-2154.

B. Spaetgens, T Pustjens, L.E.J.M. Scheepers, H.J. Janssens, S. van der Linden, A. Boonen. Knowledge, illness perceptions and stated clinical practice behaviour in management of gout: a mixed methods study in general practice. Clinical Rheumatology, Feb. 2016.

L.E.J.M. Scheepers, M. van Onna, C.D.A. Stehouwer, J.A. Singh, I.C.W. Arts, A. Boonen. Medication adherence among patients with gout: a systematic review and meta-analysis. (under revision)

L.E.J.M. Scheepers, A.M. Burden, I.C.W. Arts, B. Spaetgens, P.C. Souverein, F. de Vries, A. Boonen. Medication adherence among gout patients initiated allopurinol: a retrospective cohort study in the Clinical Practice Research Datalink. (to be submitted)

I.C.W. Arts, L.E.J.M. Scheepers, J.M.A. Wijnands, P. C. Dagnelie, S. van der Linden, A. Boonen. Overproducers' and 'underexcretors': the key to understanding the complex relation between uric acid and cardiovascular diseases? (to be submitted)

\section{Not part of the thesis}

L.E.J.M. Scheepers. Folic acid: the solution for treating asymptomatic hyperuricemia. The American Journal of Clinical Nutrition, Volume 105, Number 4 April 2017

L.E.J.M. Scheepers, J. Penders, C.A. Mbakwa, C. Thijs, M. Mommers, I.C.W. Arts. The intestinal microbiota composition and weight development in children: the KOALA Birth Cohort Study. International Journal of Obesity, Oct. 2014; 39(1): 16-25. 
198 

